\title{
Photon-Echo-Spektroskopie zur Dynamik der Solvatation in Wasser und an \\ Lipidmembran-Wasser-Grenzschichten
}

\author{
Dissertation \\ zur Erlangung des Doktorgrades \\ der Mathematisch-Naturwissenschaftlichen Fakultäten \\ der Georg-August-Universität zu Göttingen
}

vorgelegt von

Helge Bürsing

aus Gifhorn

Göttingen 2002 
D7

Referent: $\quad$ Prof. Dr. Jürgen Troe

Korreferent: Prof. Dr. Michael Buback

Tag der mündlichen Prüfung: 24. April 2002 


\section{INHALTSVERZEICHNIS}

1 Zusammenfassung 5

2 Einleitung 7

3 Phospholipidmembrane 9

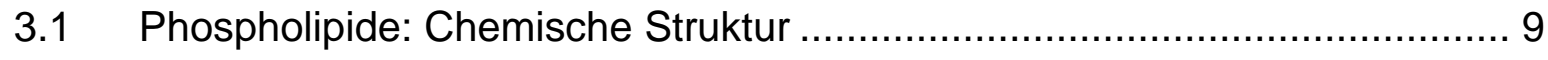

3.2 Lipiddoppelschicht (Membran) ……............................................. 11

3.3 Eigenschaften von Phospholipidmembranen: Vesikel.............................. 13

3.3.1 Phasenverhalten von Phospholipiddoppelschichten ......................... 13

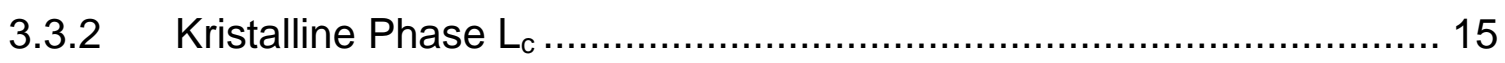

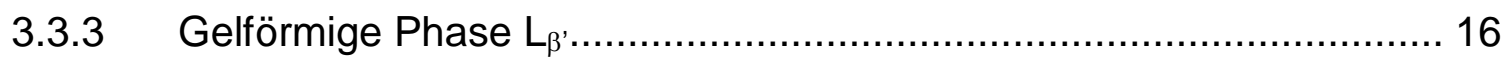

3.3.4 Zackige Phase $P_{\beta}$, (,ripple phase ") .................................................. 17

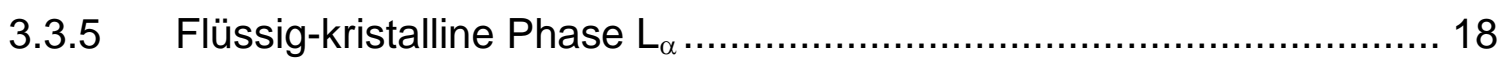

3.3.6 Hydratisierung ........................................................................ 19

3.3.7 Molekulardynamik-Simulationen ............................................... 20

3.3.8 Kommentar zu den Eigenschaften der Lipiddoppelschicht................. 21

4 Theoretische Grundlagen .................................................22

4.1 Elektronisches 2-Niveau-System in der Bloch'schen Beschreibung........... 26

4.2 Stochastische Theorie zur Resonanzbreite nach Kubo ............................. 33

4.3 Störungstheoretische Beschreibung von Vier-Wellen-Mischen (4WM) ...... 38

4.4 Das Modell der Brown'schen Oszillatoren ................................................ 43

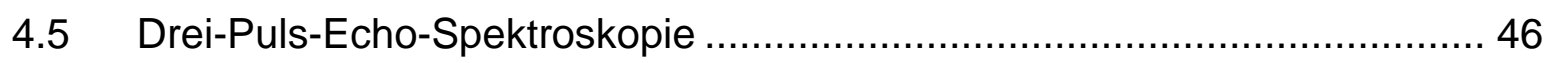

4.6 Transiente Gitter Spektroskopie ...................................................... 50 
5 Experimenteller Teil .......................................................... 53

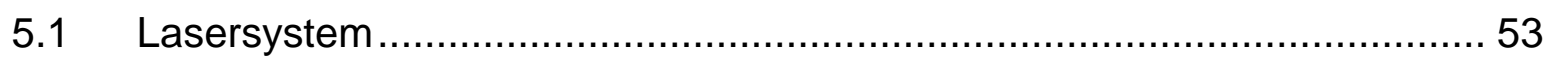

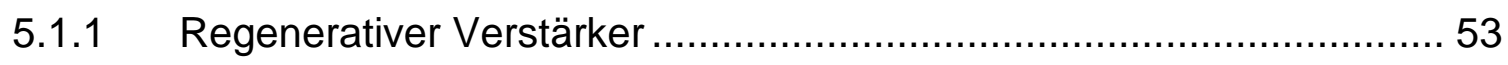

5.1.2 Nichtkollinearer optisch-parametrischer Verstärker .........................5 54

5.1.2.1 Theorie der parametrischen Verstärkung im NOPA ................... 54

5.1.2.2 Aufbau des NOPA.......................................................... 58

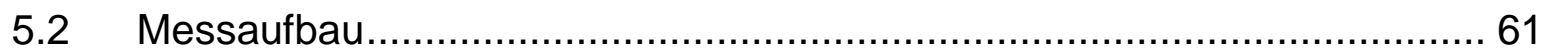

5.2.1 Aufbau der Dreiecksgeometrie............................................ 61

5.2.2 Aufbau der Boxcargeometrie................................................. 63

5.2.3 Vergleich zwischen Dreiecks- und Boxcargeometrie .......................6 65

5.3 Experimentsteuerung und Detektion der Signale ............................... 66

5.4 Temperaturstabilität im Experiment............................................. 68

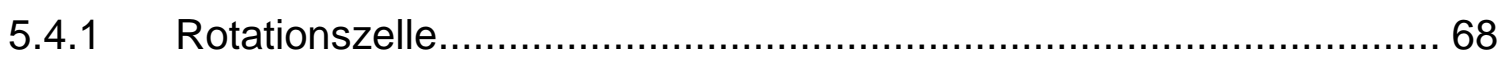

5.4.2 Untersuchung der Erwärmung der Probe im Experiment..................69 69

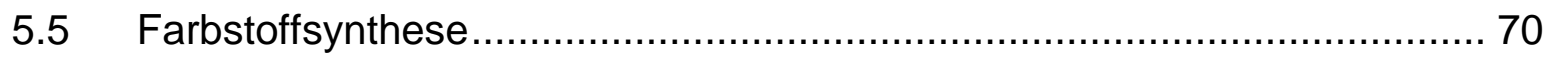

5.6 Probenmoleküle und Vesikelpräparation ........................................ 72

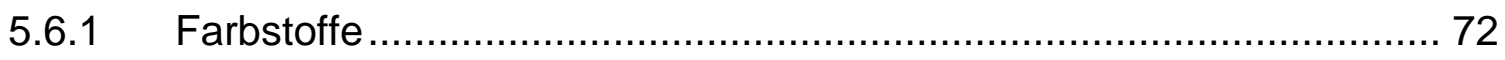

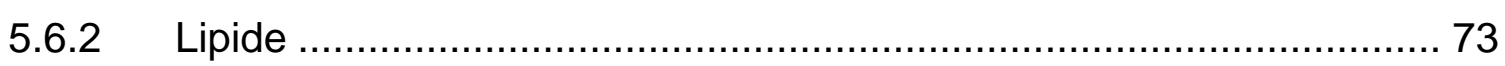

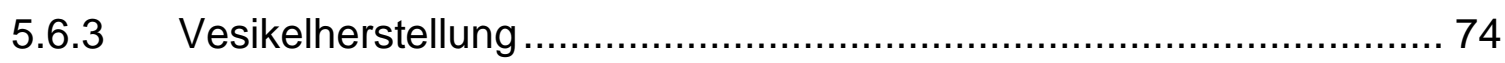

6 Ergebnisse und Diskussion ......................................................76

6.1 Allgemeine Untersuchungen der Farbstoffe und der Lipidlösungen .......... 77

6.2 Zwei-Puls-Echo-Messungen (2-PE) ........................................... 89

6.3 Drei-Puls-Echo-Peakshift-Messungen (3-PEPS) von DiDC 1 in Wasser ..... 91

6.3.1 Analyse der Solvatationskorrelationsfunktion des Wassers bei Zimmertemperatur ........................................ 91

6.3.2 Temperaturabhängigkeit der Solvatation von $\mathrm{DiDC}_{1}$ in Wasser ........ 102 
6.4 Drei-Puls-Echo-Peakshift-Messungen von $\mathrm{DiDC}_{18}$

an der Lipidmembran-Wasser-Grenzschicht.

6.4.1 Analyse der Solvatationsdynamiken an der

Lipidmembran-Wasser-Grenzschicht im Vergleich zum Wasser......

6.4.2 Diskussion der gemessenen Solvatationsdynamik

in Bezug zu Arbeiten aus der Literatur.

6.4.3 Temperaturabhängigkeit der Solvatationsdynamik an der Lipidmembran-Wasser-Grenzschicht

6.4.3.1 Temperaturabhängigkeit der Solvatationsdynamik

an der DMPC-Wasser-Grenzschicht.

6.4.3.2 Temperaturabhängigkeit der Solvatationsdynamik

an der DOPC-Wasser-Grenzschicht

6.4.3.3 Temperaturabhängigkeit der Solvatationsdynamik an der DPPC-Wasser-Grenzschicht

7 Ausblick.

8 Anhang

9 Literaturverzeichnis 


\section{ZUSAMMENFASSUNG}

Die vorliegende Arbeit beschäftigt sich mit den molekularen Mechanismen zur ultraschnellen Solvatation an wässrigen Grenzschichten von synthetischen Phospholipid-Membranen. Als experimentelle Technik, die in solchen Systemen erstmalig zum Einsatz kommt, dient die Photon-Echo-Spektroskopie. Als Sonde für dynamische Solvatation wird ein Indocarbocyanin-Farbstoff, der nicht-kovalent in der Phospholipid-Membran verankert ist und dessen optischer Chromophor an der Grenzschicht Wasser/Lipid lokalisiert ist, verwendet.

Komplementäre Untersuchungen zur Solvatationsdynamik desselben Chromophors in wässrigen Volumenphasen erlauben die eindeutige Identifizierung von grenzschicht-spezifischen Phänomenen.

Die Dynamik der Solvatation in wässrigen Volumenphasen bei Zimmertemperatur erfolgt im wesentlichen auf zwei Zeitskalen:

a.) unterhalb von etwa 1 ps beobachtet man die Relaxation kollektiver, gehinderter Translationsmoden, die für das zufällige ungestörte Wasserstoffbrücken-Netzwerk der reinen Flüssigkeit typisch sind,

b.) oberhalb von etwa $1 \mathrm{ps}$ ist die Solvatation dominiert durch die diffusive Reorientierungsdynamik der Wassermoleküle in der Umgebung des Chromophors.

Die Dynamik der Solvatation an wässrigen Grenzschichten synthetischer Phospholipid-Membranen erfolgt dagegen bei Zimmertemperatur nur auf einer Zeitskala von mehreren Pikosekunden. Ein detaillierter Vergleich zwischen grenzschicht-spezifischer Solvatation und Volumenphasen-Solvatation legt nahe, dass das für reines Wasser charakteristische Wasserstoff-Brückennetzwerk an der Oberfläche von wässrigen Phospholipid-Membranen aufgebrochen ist. 
Begleitende temperaturabhängige Studien zur dynamischen Solvatation ermöglichen den komplexen Polymorphismus synthetischer Membranen erstmalig mit ultraschnellen Strukturrelaxationen an deren Grenzschichten in Verbindung zu bringen: Im Gegensatz zum Hauptphasenübergang (zackig-gelförmig $\leftrightarrow$ flüssig-kristallin) der mit Unordnungsphänomenen im hydrophoben Inneren solcher Membranen verknüpft ist, beeinflusst der Vorphasenübergang (zackig-gelförmig $\leftrightarrow$ gelförmig) die Solvatation gerade auf Zeitskalen unterhalb 1 ps. Ein solcher Befund ist ein eindeutiger Beleg für strukturelle Änderungen in der Grenzschichtregion, die den thermodynamischen Vorphasenübergang begleiten und ist konsistent mit einer Dehydratisierung der Grenzfläche beim Übergang von der zackig-gelförmigen Phase zur gelförmigen Phase. 


\section{EinLeitung}

Phospholipid-Membranen stellen ein wichtiges Strukturelement in biologischen Systemen dar. Ihre primäre Funktion ist die räumliche Trennung von Zelle oder Zellorganellen und deren Umgebungen. Sie sind darüber hinaus die ideale Matrix für eine Vielzahl von Proteinen, die ihrerseits für die Funktion von Zellen bzw. Organellen verantwortlich sind. Bestimmte, in die Membran eingebettete Proteine übernehmen unter anderem die Aufgabe des Stofftransportes und damit den Austausch von Informationen zwischen Zellen oder deren Kompartementen. Von essentieller Bedeutung ist in diesem Zusammenhang auch der Ladungstransport (also Transport spezifischer lonen) über biologische Grenzschichten. Prototypisch für einen solchen Ladungstransport ist beispielsweise die lichtgetriebene Protonenpumpe Bacteriorhodopsin, die in Purpurmembranen von Halobakterien gefunden werden. Die primäre Absorption von Licht durch den proteineigenen Chromophor Retinal wird dazu genutzt, einen transmembranen Protonengradienten aufzubauen. Letzterer dient schließlich dazu, um in einem zweiten Membranprotein, der sogenannten ATP-Synthase, chemische Energie in Form von ATP bereitzustellen und damit die Energie des primär absorbierten Photons für die Zelle nutzbar zu machen.

Der Grenzschicht Membran-Wasser kommt beim Transport geladener oder auch dipolarer Spezies eine ganz besondere Bedeutung zu. Wassermoleküle, die an der Grenzschicht lokalisiert sind, übernehmen den Aufbau einer Hydrathülle der transportierten Spezies und ermöglichen erst deren Austritt aus der ProteinMembran-Einheit über die Grenzschicht hinweg und hinein in die wässrige Volumenphase weit oberhalb der Lipiddoppelschicht.

Der Aufbau einer solchen Hydrathülle ist schwer im Experiment zu verfolgen. Es ist aber möglich, stellvertretend für den Transport eines geladenen oder dipolaren Teilchens über die Membran, die Ladungsdichteverteilung eines unmittelbar an der Grenzschicht lokalisierten Sondenmoleküls zu verändern. So kann durch Absorption eines ultrakurzen Lichtimpulses die räumliche Lage bzw. die Größe des Dipolmoments eines solchen Sondenmoleküls extrem schnell geschaltet werden. Die Moleküle in der Umgebung der Sondenmoleküle stabilisieren daraufhin die neue Ladungsdichteverteilung durch Umorientierung ihrer eigenen Dipole. Damit ein solches Experiment gelingt, muß die Ladungsdichteänderung im Sondenmolekül schneller erfolgen als die Reorientierungsdynamik in der Umgebung. 
Experimentell kann die Dynamik der Stabilisierung einer „instantan“ erzeugten Ladungsdichtestörung verfolgt werden, indem das zeitabhängige Emissionsspektrum der Sondenmoleküle aufgezeichnet wird. Die Stabilisierung geht mit einer Verschiebung der Sondenfluoreszenz zu niedrigeren Frequenzen einher. Daher werden solche Experimente auch zeitaufgelöste Stokes-Verschiebung (oder „timedependent fluorescence Stokes shift", TDFSS) genannt.

TDFSS-Experimente sind Grundlage einer Vielzahl von Arbeiten zur dynamischen Solvatation in molekularen Flüssigkeiten. Zur Solvatationsdynamik an PospholipidMembranen gibt es zur Zeit kaum experimentelle Befunde. Es existieren TDFSSExperimente von M. Hof und Mitarbeitern zur dynamischen Solvatation auf Nanosekundenzeitskalen [1,2]. Analoge Studien zur Solvatation in inneren Wassereinschlüssen von Phospholipid-Vesikeln sowie inversen Mizellen wurden von Bhattacharyya [3-9] bzw. Levinger und Mitarbeitern [10-12] berichtet. Zur grenzschicht-spezifischen Solvatation an Lipidmembranen gibt es bislang weder experimentelle noch theoretische (z.B. auf Basis von MD-Simulationen) Befunde.

Die vorliegende Arbeit ist folgendermaßen gegliedert: Kapitel 3 beschreibt zunächst die für die Experimente wichtigsten chemischen und physikalischen Eigenschaften der Phospholipidmembranen. Ein großer Teil dieses Abschnitts liefert die Diskussionsgrundlage für die gewonnenen experimentellen Ergebnisse. Die theoretischen Grundlagen der hier durchgeführten Experimente werden in Kapitel 4 erörtert. Ein besonderer Wert ist in diesem Kapitel auf die Modelle zur Beschreibung von Solvatationsprozessen gelegt. Außerdem wird der mathematische Zusammenhang zwischen der in den Experimenten bestimmten 3-Puls-Echo-PeakshiftFunktion und der Solvatationskorrelationsfunktion dargestellt. In Kapitel 5 werden das verwendete Lasersystem und der experimentelle Aufbau beschrieben. Anschließend werden die Ergebnisse vorgestellt und diskutiert. 


\section{Phospholipidmembrane}

Im folgenden Kapitel werden Membrane, die aus Phospholipiden aufgebaut sind, vorgestellt. Zum grundlegenden Verständnis der Membranen wird im ersten Teil die chemische Struktur der Phospholipide erklärt. Diese Struktur bedingt die physikalischen Eigenschaften und damit die Ausrichtung der einzelnen Moleküle in den Membranen. Im zweiten Teil wird die spontane Ausbildung von Lipiddoppelschichten (Membranen) dargestellt, bevor im dritten Teil die in der Literatur bekannten Eigenschaften dieser Membranen diskutiert werden.

\subsection{Phospholipide: Chemische Struktur}

Lipide sind amphiphile Moleküle mit einer polaren Kopfgruppe, an die unpolare Kohlenwasserstoffketten gebunden sind [13].

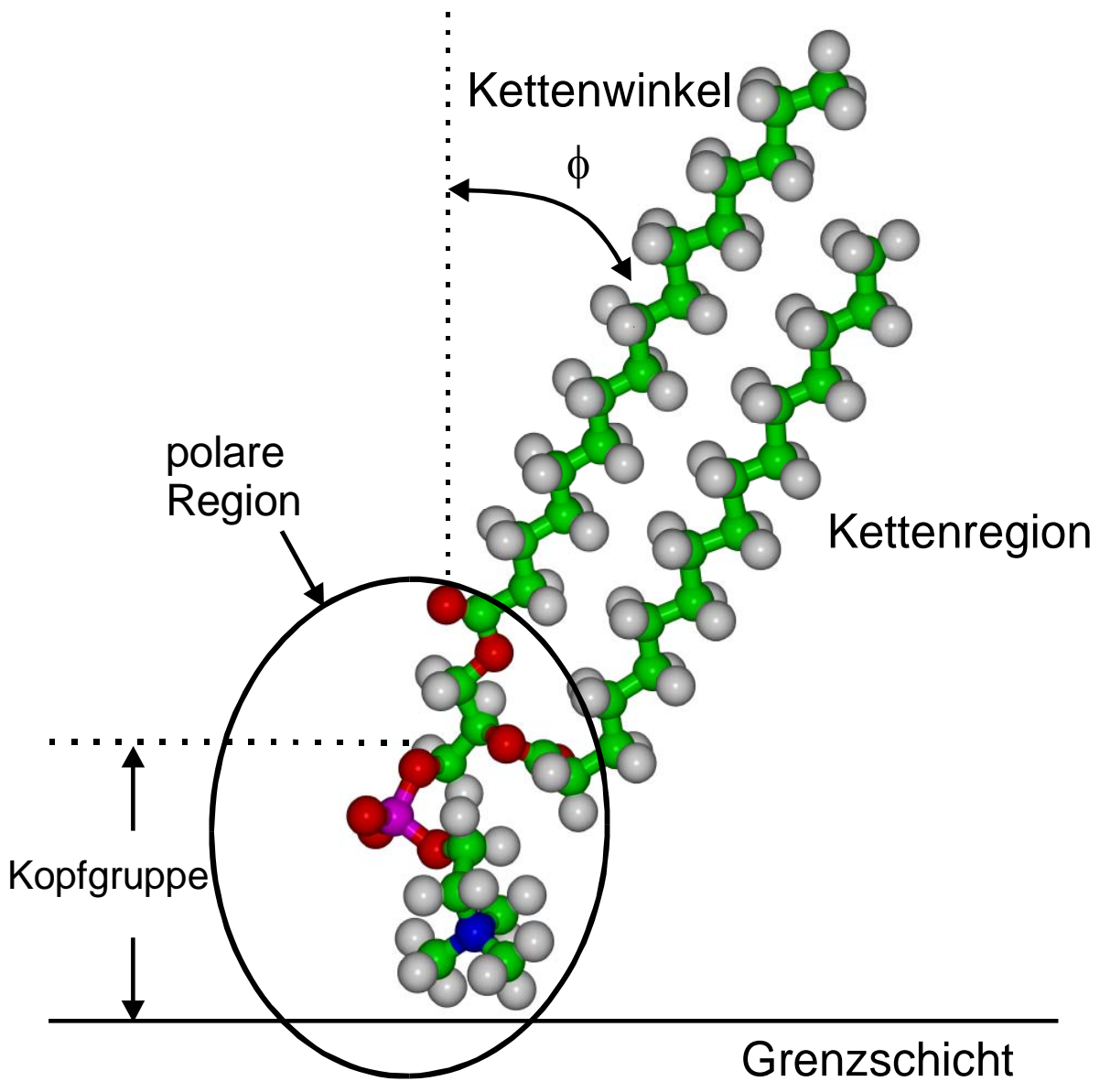

Abbildung 3.1: Unterteilung von Lipiden am Beispiel von DPPC; Kohlenstoff = grün; Wasserstoff $=$ grau; Sauerstoff $=$ rot; Phosphor $=$ violett; Stickstoff $=$ blau . 
Die Kopfgruppen unterscheiden sich in ihrer Größe und ihrer Polarität. Es gibt neutrale, einfach geladene und mehrfach geladene Kopfgruppen. Neutrale Lipidkopfgruppen kommen in der Natur nicht vor und sind daher nur synthetisch zugänglich.

Die Größe der Kopfgruppe wird durch die an sie gebundenen Substituenten bestimmt. Die natürlichen Phospholipide unterteilen sich hierbei in zwei Hauptgruppen, den Glycerophospholipiden (siehe Abbildung 3.2) und den Sphingophospholipiden [13]. Diese Phospholipide dominieren den Bereich der biologischen Membranen (Lipiddoppelschichten). In den folgenden Abschnitten wird lediglich auf die Glycerophospholipide eingegangen, da diese die in der Arbeit untersuchten Lipide darstellen.

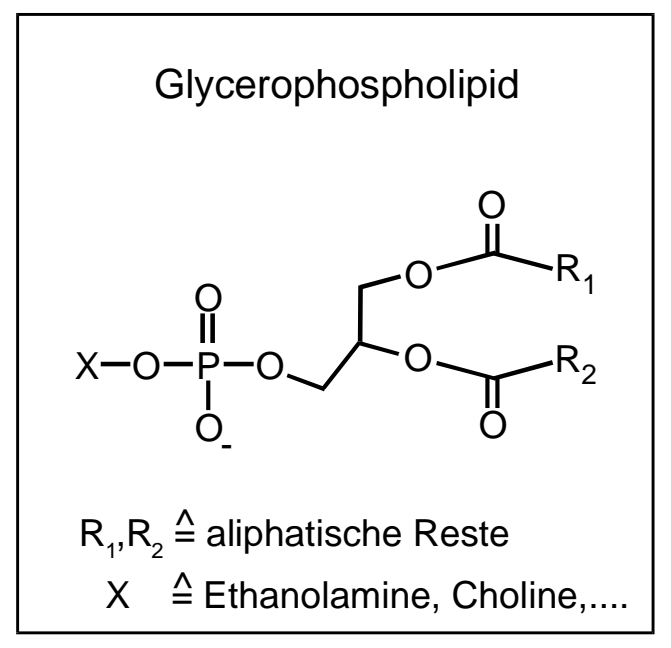

\section{Abbildung 3.2: Allgemeine Strukturformel der Glycerophospholipide}

$\mathrm{Zu}$ den wichtigsten Kopfgruppen der Glycerophospholipide zählen die Phosphatidylethanolamine, Phosphatidylcholine, Phosphatidylglycerole, Phosphatidyllinositole und Phosphatidylserine. Die Kopfgruppen werden häufig durch an sie gebundene Moleküle modifiziert. Für spektroskopische Untersuchungen können z.B. Farbstoffe gezielt durch eine kovalente Bindung mit der Kopfgruppe verbunden werden [10].

Die Kettenregion (Abbildung 3.1) besteht aus aliphatischen Kohlenwasserstoffketten, die bei den natürlichen Lipiden von den natürlich vorkommenden Carbonsäuren abstammen. Die Lipide besitzen je nach Kopfgruppe eine bzw. zwei Kohlenstoffketten. Diese Ketten kommen in verschiedenen Längen vor und können sich im Sättigungsgrad unterscheiden. $D a R_{1}$ und $R_{2}$ (siehe Abbildung 3.2) unterschiedlich sein können, gibt es eine große Vielfalt von Lipiden. 


\subsection{Lipiddoppelschicht (Membran)}

Die Kombination aus polarer Kopfgruppe und unpolarer Kette verursacht die für Lipide typischen Eigenschaften. Die polare Kopfgruppe bevorzugt polare Umgebungen, während die unpolare Kette unpolare Bereiche vorzieht. Dieser amphiphile Charakter bewirkt, dass die Lipide in reiner Form multilamellare Doppelschichten ausbilden [13]. Eine Schicht von Kopfgruppen wird in diesem Fall gefolgt von einer Schicht unpolarer Ketten. An diese schließt sich wieder eine Kopfgruppenschicht an, usw. . Diese Schichten bleiben im Wasser über lange Zeit stabil. Durch geeignete Bedingungen und Methoden lassen sich diese Strukturen aufbrechen und neue Strukturen entstehen. In Abbildung 3.3 sind drei dieser Strukturen gezeigt. Neben den abgebildeten Strukturen gibt es z.B. die bereits erwähnten multilamellaren Schichten. Die Eigenschaften der Kopfgruppe und die Form der Kette bestimmen, welche Struktur sich spontan ausbildet. Schon kleine chemische Unterschiede (z.B. im Sättigungsgrad; zwei gleiche Ketten im Gegensatz zu zwei unterschiedlichen Ketten) in den Lipiden verändern die Strukturen und die thermodynamischen Eigenschaften entscheidend.
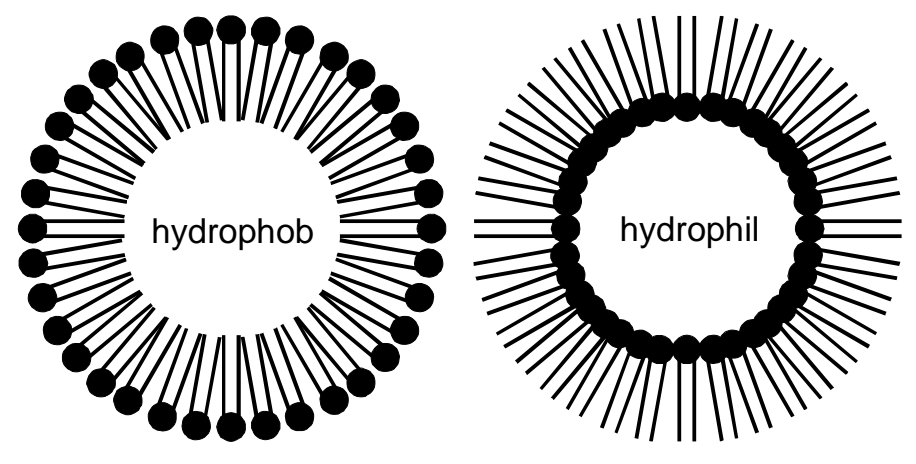

Mizelle

inverse Mizelle

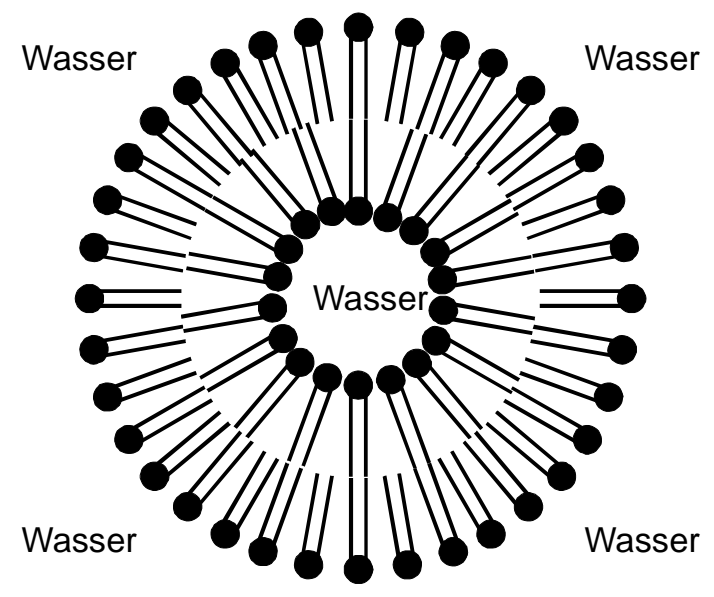

unilamellares

Vesikel

Abbildung 3.3: Strukturen von Lipiden in Lösung [13]

Eine Möglichkeit die Struktur der multilamellaren Schichten aufzubrechen ist das Zusetzen von Lösungsmitteln. Bei der Zugabe von einem unpolaren Lösungsmittel (z.B. Alkane), wird dieses Lösungsmittel im Bereich der unpolaren Ketten eingelagert. Im Gegensatz dazu brechen polare Lösungsmittel (z.B. Wasser) die Schichten an den Kopfgruppen auf. Die so entstandenen Doppelschichten zeigen mit den polaren Kopfgruppen nach außen in das Wasser. Es entstehen auf diese Weise 
auch Wassereinschlüsse. Dies sind Bereiche von Wasser, die durch die Membranschichten vom restlichen Wasser abgeschirmt sind (siehe Vesikel in Abbildung 3.3).

Um die individuellen, mesoskopischen Strukturen auf ihre speziellen Eigenschaften hin zu untersuchen, wurden in der Vergangenheit Verfahren zu ihrer gezielten Herstellung entwickelt. Zur Präparation von Vesikeln gibt es eine Vielzahl von Methoden, die spezielle Größen und Größenverteilungen erzeugen. Vesikel werden auf Grund ihrer Größe und der Anzahl der Schichten eingeteilt. Es wird unterschieden in unilamellare und multilamellare Vesikel. Die Grobeinteilung der Größe von unilamellaren Vesikeln beschränkt sich auf kleine (,small unilamellar vesicles“, SUV), große ("large unilamellar vesicles“, LUV) und sehr große (,giant vesicles", gigantische Vesikel) Vesikel. Während die SUVs eine Größe von weniger als $100 \mathrm{~nm}$ besitzen, und LUVs größer als $100 \mathrm{~nm}$ sind, erreichen gigantische Vesikel eine Größe von $20 \mu \mathrm{m}$ bis $100 \mu \mathrm{m}$.

Zur Herstellung von gigantischen Vesikeln wird z.B. eine Elektroformationsmethode angewandt [14, 15]. Eine recht alte Methode ist die Ultraschallpräparation von Vesikeln. Die dadurch entstehenden Vesikel haben eine breite Größenverteilung. Die Dauer der Ultraschallbestrahlung bestimmt maßgeblich, inwieweit die zunächst großen multilamellaren Vesikel zerbrechen und sich kleinere Vesikel bilden. SUV werden auf diese Weise durch langes Einwirken von Ultraschallstrahlung auf die Lösung hergestellt.

Vesikel mit einer schmalen Größenverteilung werden dagegen mittels Extrudieren durch kleinporige Filter hergestellt. Bei diesem Verfahren bestimmt die Größe der Poren in einem Polycarbonatfilter und der angewandte Druck auf die Lösung [16] die mittlere Größe der Vesikel. Diese Methode wurde bei den Experimenten in der vorliegenden Arbeit angewandt. Die Lösungen wurden durch einen $100 \mathrm{~nm}$ Filter extrudiert und zeichneten sich durch eine Größenverteilung mit einer Halbwertsbreite von ca. $20 \mathrm{~nm}$ aus. Das Maximum dieser Verteilung lag bei ca. $80 \mathrm{~nm}$ [17]. 


\subsection{Eigenschaften von Phospholipidmembranen: Vesikel}

Im nun folgenden Abschnitt werden die bekannten Eigenschaften von Lipiddoppelschichten vorgestellt. Dabei werden insbesondere die speziellen Eigenschaften der unilamellaren Vesikel, die in den Experimenten eingesetzt werden, hervorgehoben. Es werden jedoch auch Untersuchungen von multilamellaren Schichten berücksichtigt.

Die physikalisch-chemischen Eigenschaften von Phospholipidmembranen sind von Bedeutung für biologische Prozesse, wie zum Beispiel dem lonentransport [18, 19] durch eine Lipidmembran. In der Vergangenheit sind daher viele Arbeiten publiziert worden, in denen diese komplexen Systeme mittels unterschiedlichster Methoden untersucht wurden. Auf den folgenden Seiten wird nur ein Überblick über die für die durchgeführten Experimente wesentlichen Erkenntnisse gegeben. Eine ausführlichere Beschreibung ist den angegebenen Literaturstellen zu entnehmen.

\subsubsection{Phasenverhalten von Phospholipiddoppelschichten}

Phospholipiddoppelschichten besitzen ein einzigartiges und hochkomplexes thermodynamisches Phasenverhalten. Zur Aufklärung des Polymorphismus wurden Untersuchungen an reinen Lipiden in Form von Langmuir-Blodgett-Filmen an Lipid/Wasser - Gemischen und an Lipid/Lipid/Wasser-Gemischen durchgeführt $[9,10]$. Auch der Einfluss von Fremdmolekülen wie z.B. Cholesterol [22-25] oder Melittin [26] auf den Polymorphismus von Lipiden sind gerade bezüglich biologischer Membrane von Interesse.

Es wurden vier Phasen beobachtet. Die Phasenübergänge zwischen diesen vier Phasen sind durch Messungen der Wärmekapazität sowohl in unilamellaren Vesikeln, als auch in multilamellaren Schichten zu beobachten. Die unterschiedlichen thermodynamischen Phasen variieren in der mikroskopischen Anordnung der Lipide innerhalb der Doppelschicht (siehe Abbildung 3.4). 


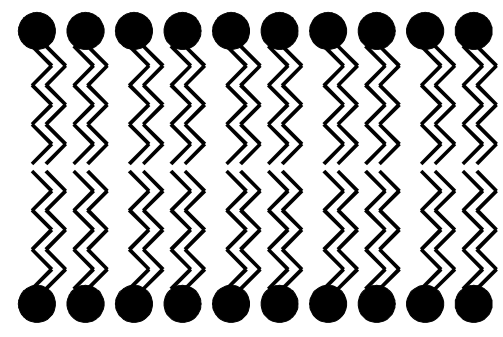

$\mathrm{L}_{c}$
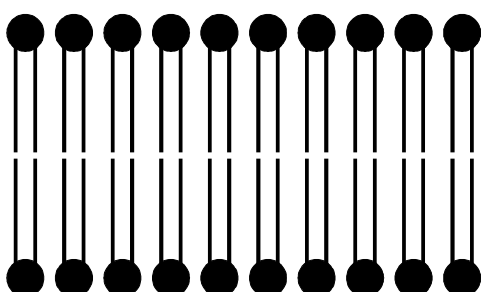

$\mathrm{L}_{\beta}$
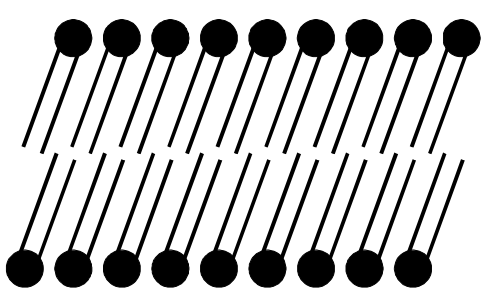

$L_{\beta}$

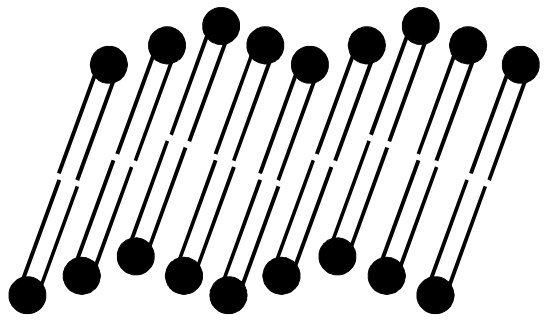

$\mathrm{P}_{\beta}$,

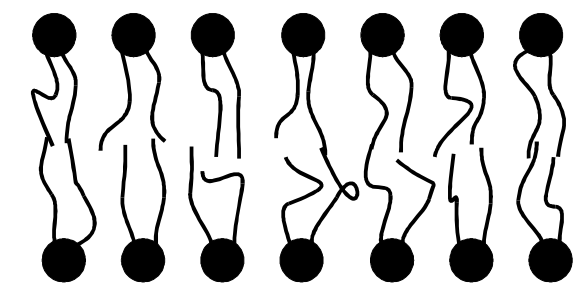

$\mathrm{L}_{\alpha}$

Abbildung 3.4: Strukturen der verschieden Lipidphasen

Die Lipidphasen werden nach einer Nomenklatur von Tardieu, Luzzati und Reman [27] benannt. Die Großbuchstaben bezeichnen die weitreichende Ordnung (1-D, 2-D, 3-D Gitter). Der Buchstabe L steht für eindimensional lamellares, $P$ für ein zweidimensional zentriertes und $\mathrm{C}$ für ein dreidimensional kristallines Gitter. Die griechischen Indizes beschreiben die Ordnung in den Lipidketten. $\alpha$ deutet eine mit Flüssigkeiten vergleichbare Ordnung an, während $\beta$ und $\beta$ ' für teilweise geordnete Ketten stehen. Das Apostroph bezieht sich auf einen Kettenwinkel von ungleich $0^{\circ}$ (siehe Abbildung 3.1). Ein eindimensional lamellares Gitter bedeutet in Abbildung 3.4, dass die Lipidkopfgruppen in einer Linie angeordnet sind, während im zweidimensional zentriertem Gitter die Kopfgruppen abweichend von der Linie in eine weitere Dimension hineinragen und z.B. eine Zackenanordnung $\left(P_{\beta}\right)$ einnehmen.

Im Folgenden werden die einzelnen Strukturmerkmale und Eigenschaften der Phasen herausgearbeitet und anschließend einige Theorien vorgestellt. Eine Zusammenfassung über die Ausrichtung der Lipide findet sich in Referenz [28]. In Abbildung 3.4 sind die vorgestellten Lipidphasen schematisch mit der zugehörigen Nomenklatur gezeichnet. 


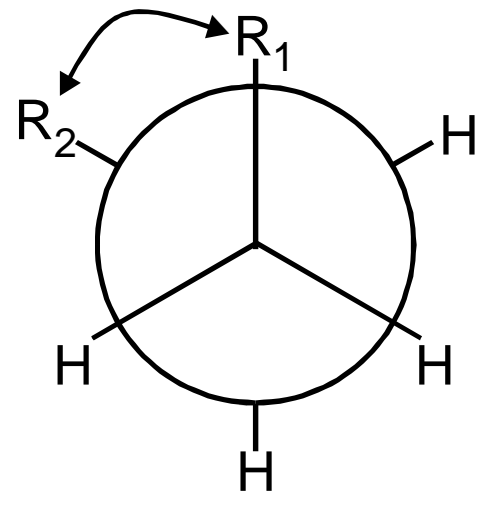

gauche

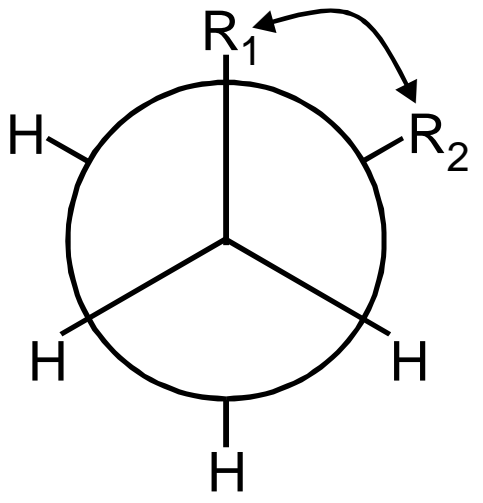

gauche $^{+}$

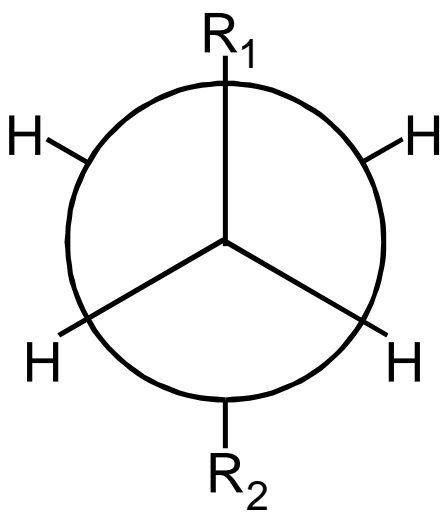

trans

Abbildung 3.5: Bezeichnungen der Konformationen der Kohlenstoffketten: Die Aufsicht auf eine Bindung der Kette ist aufgezeichnet. Die Reste $R_{1}$ (vorne) und $R_{2}$ (hinten) sind die Teile der Kette, die an die betrachtete Bindung anschließen.

\subsubsection{Kristalline Phase $L_{c}$}

Die kristalline Phase $L_{c}$ ist stabil im unteren Temperaturbereich. Auf Grund der langsamen Umwandlung [29] der gelförmigen Phase $L_{\beta}$, in die kristalline Phase $L_{c}$ (Umwandlung erfolgt innerhalb Tagen, Wochen oder Monaten) und damit langwierigen Präparation wurde sie erst 1980 von Chen erstmals beobachtet [30]. Seit ihrer Entdeckung kann diese Phase trotz weiterer Wärmekapazitätsmessungen und Röntgenbeugungsexperimenten nur unvollständig beschrieben werden [31, 32]. So ist nicht geklärt, ob die bis heute in multilamellaren Schichten beobachtete Phase auch in unilamellaren Vesikeln vorhanden ist. Die Bezeichnung " $\mathrm{L}_{c}$ " beinhaltet das bislang unvollständige Wissen, denn $\mathrm{L}$ steht für eine eindimensionale Ordnung, während das hier inkonsequenter Weise als Index verwendete große $\mathrm{C}$ in der oben vorgestellten Nomenklatur eine dreidimensionale kristalline Ordnung bezeichnet. Neue Experimente von Utoh und Okubo [33] weisen auf eine schnellere Präparation dieser Phase hin. Die kristalline Phase konnte bei diesen Experimenten mit L- $\alpha$ Dibehenoyl-Phosphatidylcholine (DBPC; Kohlenstoffkette mit $C_{n}=22$ ) direkt hergestellt werden, ohne die Probe für lange Zeit bei tiefen Temperaturen zu lagern. 
Die bisherigen Experimente lassen folgende Beschreibung der kristallinen Phase zu:

1.) Die Phase besitzt eine höhere Ordnung der Ketten als die der $L_{\beta}$, Phase [34]. Alle Bindungen der Ketten sind in trans-Konformation (Abbildung 3.5).

2.) Die von einer Kette beanspruchte Fläche ist im Vergleich zu den übrigen drei Phasen am geringsten [35].

3.) Ohne ein eindeutiges Experiment wird angenommen, dass es sich um eine orthorhombische Kristallgitteranordnung handelt, während von anderen Arbeitsgruppen eine trikline Anordnung postuliert wird [35].

4.) Rotationen, die mit ${ }^{1} \mathrm{H}-\mathrm{NMR}$-Spektroskopie messbar sind, sind im Vergleich zur gelförmigen Phase eingefroren. Hierzu gehört sowohl die Torsion der C-C-Bindungen als auch die Rotation der Cholin-Kopfgruppe. Einzig die Rotation der $\mathrm{N}\left(\mathrm{CH}_{3}\right)_{3}-$ Gruppe ist weiterhin zu beobachten [36].

Eine Erwärmung der kristallinen Phase $L_{c}$ führt je nach Kettenlänge zu einer Umwandlung in die gelförmige Phase $L_{\beta}$, oder einer direkten Umwandlung in die zackige Phase $\mathrm{P}_{\beta}$, bzw. in die flüssig-kristalline Phase $\mathrm{L}_{\alpha}$. Erneutes Abkühlen der Lipide aus der flüssig-kristallinen Phase $L_{\alpha}$ präpariert jedoch zunächst die $P_{\beta}$, und $L_{\beta}$, Phase, bevor sich die $L_{c}$-Phase nach längerem Warten bildet. Der Phasenübergang von der kristallinen Phase $L_{c}$ in die gelförmige Phase $L_{\beta}$, wird auch unterer Phasenübergang („subtransition“) genannt. Untersuchungen des Übergangs haben gezeigt, dass sich die kristalline Phase $L_{c}$ aus der metastabilen Phase $L_{\beta}$, bildet. Die Lipide durchlaufen hierbei mehrere metastabile Zustände. Die hierfür benötigte Zeit variiert je nach Kettenlänge und ist bislang nicht genau charakterisiert [31]. Ähnlich wie der Vorphasenübergang $\left(L_{\beta}, \rightarrow P_{\beta}\right.$ ) wird der untere Phasenübergang mit einer Umorientierung der Kopfgruppen in Zusammenhang gebracht.

\subsubsection{Gelförmige Phase $L_{\beta}$,}

Die gelförmige Phase ist nach der flüssig-kristallinen Phase $L_{\alpha}$ die am längsten bekannte Phase. Die Lipidketten sind in dieser Phase vollständig gestreckt, d.h. in einer all-trans-Konformation [37]. Die gelförmige Phase unterscheidet sich von der kristallinen Phase durch eine Rotationsunordnung, d.h. die Lipidketten sind in Bezug auf ihre Orientierung zueinander nicht vollständig geordnet. Einige Lipide bevorzugen die $L_{\beta}$-Phase, in welcher der Kettenwinkel $\phi$ gleich $0^{\circ}$ ist. Die meisten Lipide ordnen 
ihre Ketten jedoch in einem Winkel $\phi$ ungleich $0^{\circ}$ an [27]. Diese Lipide in der $L_{\beta}$, -Phase besitzen auch eine im Vergleich zur $L_{\beta}$-Phase stärker hydratisierte Kopfgruppe (Abbildung 3.4). Die bevorzugte Anordnung scheint somit kopfgruppenspezifisch zu sein.

Die Gitterstruktur der aliphatischen Ketten ändert sich bei Erniedrigung der Temperatur von zunächst quasi-hexagonal in der Nähe des Vorphasenübergangs zu orthorombisch [38]. Es existieren trotz dieser Gitteranordnung noch gehinderte Kettenbewegungen mit Korrelationszeiten von $10^{-5}-10^{-7} \mathrm{~s}$ wie NMR-Messungen [39] und STESR - Experimente [40] gezeigt haben. Die von einer Kette beanspruchte Fläche nimmt im Vergleich zur kristallinen Phase nur minimal zu [34].

Eine Erwärmung der gelförmigen Phase führt in Abhängigkeit von der Kettenlänge zu einem Phasenübergang in die zackige $P_{\beta}$,-Phase bzw. in die flüssig-kristalline Phase $L_{\alpha}$. Die Temperaturdifferenz zwischen Vorphasenübergang $\left(L_{\beta} \rightarrow P_{\beta}\right)$ und Hauptphasenübergang $\left(P_{\beta}, \rightarrow L_{\alpha}\right)$ bei Phosphocholinlipiden mit gesättigten, aliphatischen Ketten reduziert sich kontinuierlich von $\Delta T=12^{\circ}$ bei DMPC (gesättigte $\mathrm{C}_{14}$-Ketten als aliphatische Reste) bis zu einem Verschmelzen der beiden Übergänge zu einem Übergang bei einer Kettenlänge von 24 Kohlenstoffatomen [41, 42].

\subsubsection{Zackige Phase $\mathbf{P}_{\beta}$, (,ripple phase“)}

In neuerer Zeit wurden viele Experimente zur Erforschung dieser Phase durchgeführt, da sie auf Grund ihrer besonderen Struktur für das allgemeine Verständnis von Lipiddoppelschichten von Bedeutung ist. Röntgenbeugungsexperimente von Shipley und Mitarbeitern [34, 35, 43, 44] an multilamellaren Schichten weisen bei kleinem Beobachtungswinkel zusätzliche Beugungslinien auf, die auf ein zweidimensionales Gitter hindeuten. Trotz gegensätzlicher Beobachtungen [45] können spätere elektronenmikroskopische Aufnahmen von eingefrorenen Schichten der $\mathrm{P}_{\beta}$,-Phase dies belegen [46, 47]. Die beobachteten Zacken („ripple“) bilden sich in Abhängigkeit vom Wassergehalt. Die räumliche Periode der Zacken ändert sich bei Phosphocholinen von $164 \AA$ bis zu $145 \AA$ mit steigendem Wassergehalt [48].

Ein weiterer Diskussionspunkt bei der Struktur dieser Phase war in der Vergangenheit der Kettenwinkel $\phi$. Während die ursprüngliche Analyse von Tardiue [27] einen Kettenwinkel ungleich $0^{\circ}$ vermuten lässt, haben Rand und Mitarbeiter [49] in Röntgenbeugungsexperimenten einen Übergang von gewinkelten 
zu nicht gewinkelten Ketten ermittelt. Neueste Ergebnisse der Infrarotspektroskopie von Le Bihan [50] belegen jedoch nur eine minimale Abnahme des Kettenwinkels von $28^{\circ}$ in der gelförmigen Phase zu $24^{\circ}$ in der zackigen Phase bei DPPC Lipiden.

Die Ketten nehmen in dieser Phase eine all-trans-Geometrie mit einer leichten Zunahme der gauche-Konformation im Vergleich zur gelförmigen Phase $\mathrm{L}_{\beta}$, ein (Abbildung 3.5). Im Mittel werden je Kette ein bis zwei gauche-Konformationen beobachtet $[38,51,52]$.

Der Übergang von $L_{\beta}$, nach $\mathrm{P}_{\beta}$, ist nach heutigem Stand von einer Neuordnung der Lipidkopfgruppen und nur in geringem Maße von einer Neuordnung der Ketten bestimmt. So ist die Umwandlungsenthalpie unabhängig von der Kettenlänge [13]. Die Volumenzunahme der Doppelschicht am Übergang ist für Kettenlängen von 13 bis 20 Kohlenstoffatomen konstant [53]. Einzig der Wassergehalt und die Aktivität des Wasser, haben einen Einfluss auf die Lage und die Enthalpie des Überganges. Es wird kein Übergang für Lipide bei einem Wassergehalt von weniger als 20 Gewichtsprozent beobachtet [44]. In diesem Fall wird die gelförmige Phase $L_{\beta}$ ' direkt in die flüssig-kristalline Phase $L_{\alpha}$ umgewandelt.

\subsubsection{Flüssig-kristalline Phase $\mathrm{L}_{\alpha}$}

Die flüssig-kristalline Phase ist für die Biologie von Bedeutung, da sie im physiologisch relevanten Temperaturbereich thermodynamisch stabil ist. Sie ist aus diesem Grund auch am gründlichsten untersucht.

Röntgenbeugungsexperimente haben eine hexagonale Gitterstruktur aufgezeigt [35]. Die Ketten zeigen in dieser Phase eine starke Zunahme der gauche-Konformation im Vergleich zu den bisher diskutierten Phasen auf. Die Ordnung innerhalb des unpolaren Kerns der Doppelschicht nimmt im Vergleich zur gelförmigen Phase um Größenordnungen ab und ist eher mit einer Flüssigkeit zu vergleichen als mit einer Gitteranordnung im Festkörper. Ebenso sind Rotationen innerhalb der Ketten zu beobachten. Diese Rotationen sind schneller als in den anderen Phasen [13]. Der Hauptphasenübergang $\left(P_{\beta}, \rightarrow L_{\alpha}\right)$ wird dementsprechend maßgeblich von den Ketten bestimmt. Das Volumen pro Lipid nimmt im Vergleich zur gelförmigen Phase stark zu. Diese Volumenzunahme $\Delta \mathrm{V}$ ist kettenlängenabhängig und nimmt mit wachsender Kettenlänge zu [53]. Ebenso nimmt die Umwandlungsenthalpie mit der Kettenlänge zu. Verglichen wird dieser Übergang daher mit dem Rotationsübergang von Paraffinen [54]. 


\subsubsection{Hydratisierung}

Die Hydratisierung der Kopfgruppen ist von besonderer Bedeutung für den lonentransport durch die Membran. Röntgenbeugungsexperimente haben des Weiteren gezeigt, dass die Hydratisierung die Ausbildung und die Strukturen der Phasen beeinflusst. So bildet sich die zackige Phase $P_{\beta}$, nur bei einem Wasser-Lipid-Konzentrationsverhältnis von mindestens 11:1 [44]. In den Arbeiten von Shipley und Mitarbeitern wird aus Röntgenbeugungsexperimenten in Abhängigkeit vom Wasser-Lipid-Verhältnis folgender maximaler Hydratisierungsgrad der einzelnen Lipide für die verschiedenen Phasen bestimmt: $L_{c}\left(11: 1 \mathrm{H}_{2} \mathrm{O} / \mathrm{Lipid}\right)$; $\mathrm{L}_{\beta^{\prime}}$ (19:1 $\mathrm{H}_{2} \mathrm{O} /$ Lipid); $\mathrm{P}_{\beta^{\prime}}$ (19:1 $\left.\mathrm{H}_{2} \mathrm{O} / \mathrm{Lipid}\right) ; \mathrm{L}_{\alpha}\left(25: 1 \mathrm{H}_{2} \mathrm{O} / \mathrm{Lipid}\right)$. Diese Werte stimmen jedoch nicht mit den Arbeiten von Inoko [45] überein, der eine wesentliche Änderung der Hydratisierung am Vorphasenübergang beobachtet. Die obigen Messungen wurden alle an multilamellaren Schichten durchgeführt und beinhalten für die Berechnung der Hydratisierung unterschiedliche Modelle der einzelnen Schichten. Die Übertragbarkeit zu unilamellaren Vesikeln muss nicht gewährleistet sein. Insbesondere MD-Simulationen haben einen viel geringeren Hydratisierungsgrad in der flüssig-kristallinen Phase von 11-16 Wassermolekülen pro Lipid berechnet [25]. Eine direkte Messung der Hydratisierung der Kopfgruppe war bislang nicht möglich. Die hydratisierte kristalline Phase hat zum Beispiel große Ähnlichkeit mit der wasserfreien kristallinen Phase. Da die Messungen von Shipley und Mitarbeiter [34, 35] einen geringen Hydratisierungsgrad bestätigen und die Packungsdichte dieser Phase im Vergleich zu allen anderen Phasen die Höchste ist, wird diese Phase als am geringsten hydratisiert angesehen. Daraus folgt, dass der untere Phasenübergang mit einer Zunahme der Hydratisierung verbunden ist.

Eine Interpretation hinsichtlich des Hydratisierungsgrades der gelförmigen und zackigen Phase ist aufgrund der offensichtlichen Diskrepanz der existierenden Experimente erschwert. Neue Infrarotexperimente von Le Bihan und Pezolet [50] deuten auf eine weitere Zunahme der Hydratisierung am Vorphasenübergang hin. Experimente und Interpretationen von Cevz [55] ergeben ebenso, dass die zackige Phase stärker hydratisiert ist als die gelförmige Phase. Die stärkere Hydratisierung wird von Cevz als Triebkraft für das Bilden der Zacken angesehen. Des Weiteren existiert die zackige Phase nur bei einigen speziellen Kopfgruppen. Auch eine Abnahme des Wassergehalts unter 11 Wassermolekülen pro Lipid verhindert die Ausbildung dieser Phase. Ein weiterer Hinweis auf die Bedeutung der 
Wassermoleküle beim Aufbau dieser Phase ist der direkte Bezug zwischen Wassergehalt und Periode sowie Amplitude der Zacken (siehe Kapitel 3.3.4). Alle Hinweise deuten beim Vorphasenübergang somit auf einen Kopfgruppeneffekt hin und eine Zunahme der Hydratisierung beim Übergang von $L_{\beta}$, nach $P_{\beta^{\prime}}$.

Die flüssig-kristalline Phase und die zackige Phase lassen nur einen groben Vergleich zu, denn das Gitter ändert sich von einer zweidimensionalen $\left(P_{\beta^{\prime}}\right)$ zu einer eindimensionalen Anordnung $\left(\mathrm{L}_{\alpha}\right)$. Welche Auswirkungen dies auf die Hydratisierung hat, lässt sich nur erahnen. Ein Vergleich in einer Dimension würde eine deutlich vergrößerte Oberfläche für die flüssig-kristalline Phase bedeuten, während die zweite Dimension diesen Unterschied in der Oberflächengröße verringert. Unterschiedliche Messungen der Änderungen des Hydratisierungsgrades am Hauptphasenübergang erschweren das Verständnis dieses Übergangs weiter. Einzig die qualitative Aussage, dass der Hauptphasenübergang von den Ketten bestimmt ist, deutet auf keine großen Änderungen bei der Hydratisierung hin. Diese These wird durch einen vom Wassergehalt unabhängigen Phasenübergang gestützt.

Zusammenfassend nimmt die Hydratisierung, der obigen Diskussion entsprechend, am unteren Übergang und am Vorphasenübergang entscheidend zu, während am Hauptphasenübergang hauptsächlich Veränderungen im hydrophoben Kern der Doppelschicht stattfinden.

\subsubsection{Molekulardynamik-Simulationen}

Zu der flüssig-kristallinen Phase existieren eine Vielzahl von molekular-dynamischen Simulationen (MD-Simulationen) [25, 56-66]. Diese Simulationen versuchen die Dynamiken der Membran, die Solvatation an der Grenzschicht und die Geometrie zu bestimmen. In Abbildung 3.6 sind Geometrieberechnungen der einzelnen hydratisierten Lipidphasen in einem dreidimensionalen Bild dargestellt [61]. Diese Berechnungen von Schulten und Mitarbeitern zeigen im Unterschied zu den meisten anderen Simulationen drei Phasen. Die unterschiedliche Ordnung der Phasen ist deutlich zu erkennen. Auch die Schichtdicke ändert sich beim Übergang von der kristallinen Phase zur flüssig-kristallinen Phase. Des Weiteren fällt die Ordnung der Wassermoleküle an der Grenzschicht der kristallinen Phase auf. 


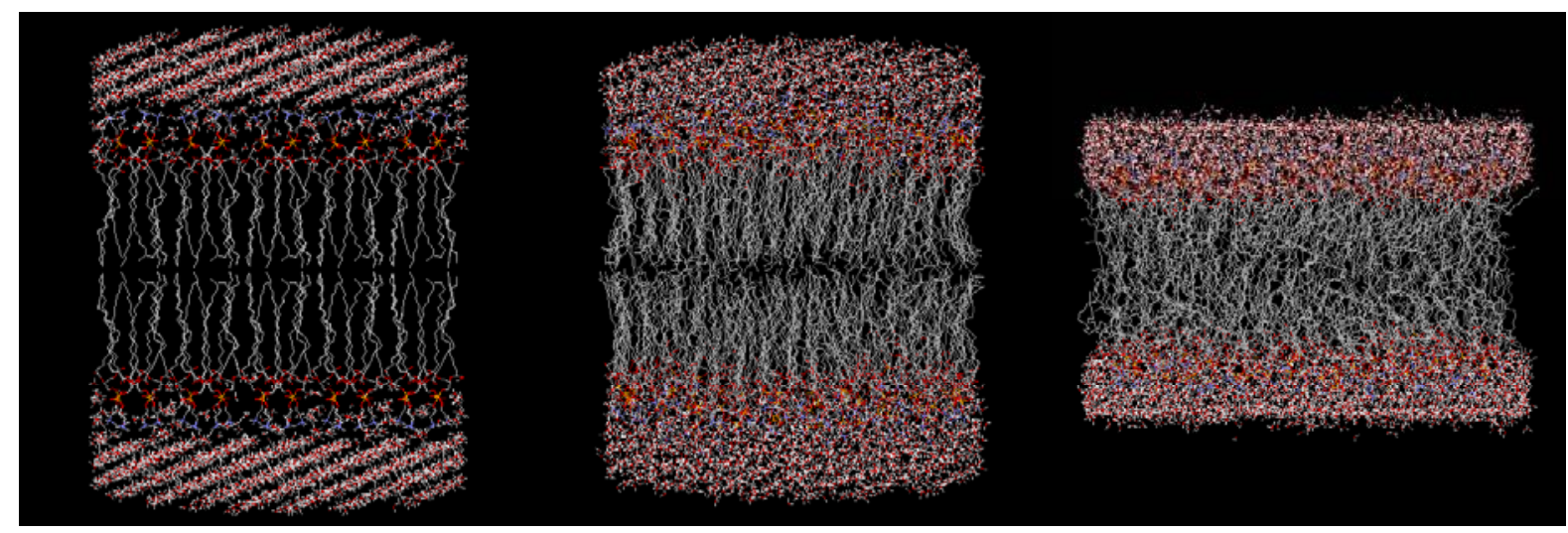

Abbildung 3.6: Kristalline Phase (links); gelförmige Phase (Mitte); flüssig-kristalline Phase (rechts) von DPPC-Lipiddoppelschichten ermittelt aus molekulardynamischen Simulationen [61]

Neben diesen qualitativen Aussagen über die Lipiddoppelschicht, geben solche Simulationen auch Informationen über die Polarisation des Wassers im Bereich der gesamten Grenzschicht und über die Eindringtiefe des Wasser in die Grenzschicht [63]. Tieleman und Mitarbeiter teilen die Lipidmembran auf Grund ihrer Simulationen von außen nach innen in vier Regionen ein [25]. Die erste Region betrifft den Bereich, in dem Wasser von den Lipidkopfgruppen beeinflusst wird. In der zweiten Region nimmt die Wasserdichte an der Grenzschicht bis auf Null ab. Die dritte Region ist bestimmt von einer hohen Kettendichte und wenig „freiem Volumen“. In der vierten Region ist die Dichte insgesamt gering. Die MD-Simulationen werden allgemein durch die Rechenzeit und die Rechnergeschwindigkeit begrenzt, so dass bei Systemen dieser Größe die realen Zeiten der Simulationen heutzutage unter einer Nanosekunde liegen. Diese Zeiten reichen zur Beschreibung der flüssigkristallinen Phase aus. Die Dynamiken der anderen Phasen sind dagegen langsamer, so dass detaillierte Berechnungen schwer durchführbar sind.

\subsubsection{Kommentar zu den Eigenschaften der Lipiddoppelschicht}

In diesem Kapitel wurden die verschiedenen Phasen der Lipidmembran vorgestellt. Für die durchgeführten Messungen und die anschließende Diskussion sind insbesondere die Eigenschaften in Bezug auf den Vorphasenübergang von Bedeutung, da sich die Solvatationskorrelationsfunktion (Kapitel 6.4.3 Ergebnisse und Diskussion) an diesem Übergang stark verändert. Die Hydratisierung und die MD-Simulationen sollen neben den strukturellen Beobachtungen eine Diskussionsgrundlage für die Erklärung der beobachteten Unterschiede zwischen gelförmiger und zackiger Phase bilden. 


\section{THEORETISCHE GRUNDLAGEN}

In diesem Kapitel wird das Phänomen der dynamischen Solvatation anhand eines Zwei-Niveau-Systems erklärt und deren Zusammenhang mit dem Prozess der Phasenrelaxation erläutert. Experimentelle Ansätze zur Charakterisierung der Dynamik der Phasenrelaxation und der Solvatation werden eingehend beschrieben. Die Antwort eines vielatomigen Moleküls (z.B. eine Farbstoffsonde) auf einen optischen Lichtimpuls kann zunächst vereinfacht in einem elektronischen ZweiNiveau-System beschrieben werden (Abbildung 4.1). Die zeitliche Entwicklung nach der optischen Anregung dieses Zwei-Niveau-Systems in Anwesenheit einer fluiden Umgebung ist jedoch nicht nur vom systemeigenen Hamiltonoperator bestimmt, sondern auch stark an die Umgebungsdynamik (Bad) gekoppelt. Eine vollständige und quantitative Beschreibung der zeitlichen Entwicklung muss sowohl Informationen über die Dynamik im System als auch über die Dynamik der Umgebung beinhalten.

In Abbildung 4.1 a.) ist das Zwei-Niveau-System bestehend aus elektronischem Grund- und angeregtem Zustand in Abhängigkeit einer verallgemeinerten Lösungsmittelkoordinate q dargestellt. Die Koordinate q umfasst sämtliche molekularen Freiheitsgrade des Bades, die an den optischen Übergangsdipol $\mu$ koppeln. Fluktuationen des Bades in der Koordinate q führen damit zu Fluktuationen in der elektronischen Energielücke $\hbar \omega$. Thermische Anregungen (d.h. Fluktuationen im thermischen Gleichgewicht) in der Koordinate q führen dadurch bereits zur Verbreiterung des linearen elektronischen Absorptionsspektrums.

Die elektronische Anregung mit einem Laserpuls bringt das System in den angeregten Zustand $|e\rangle$ (Abbildung $4.1 \mathrm{~b}$.)). Dieser unterscheidet sich vom Grundzustand $|\mathrm{g}\rangle$ durch eine veränderte Ladungsverteilung. Die Umgebung befindet sich somit im Nicht-Gleichgewicht bezüglich seiner Koordinaten q. Die Folge ist eine Bewegung entlang dieser Moden in Richtung der Gleichgewichtskonfiguration bezüglich der Ladungsdichteverteilung von $|\mathrm{e}\rangle$. 

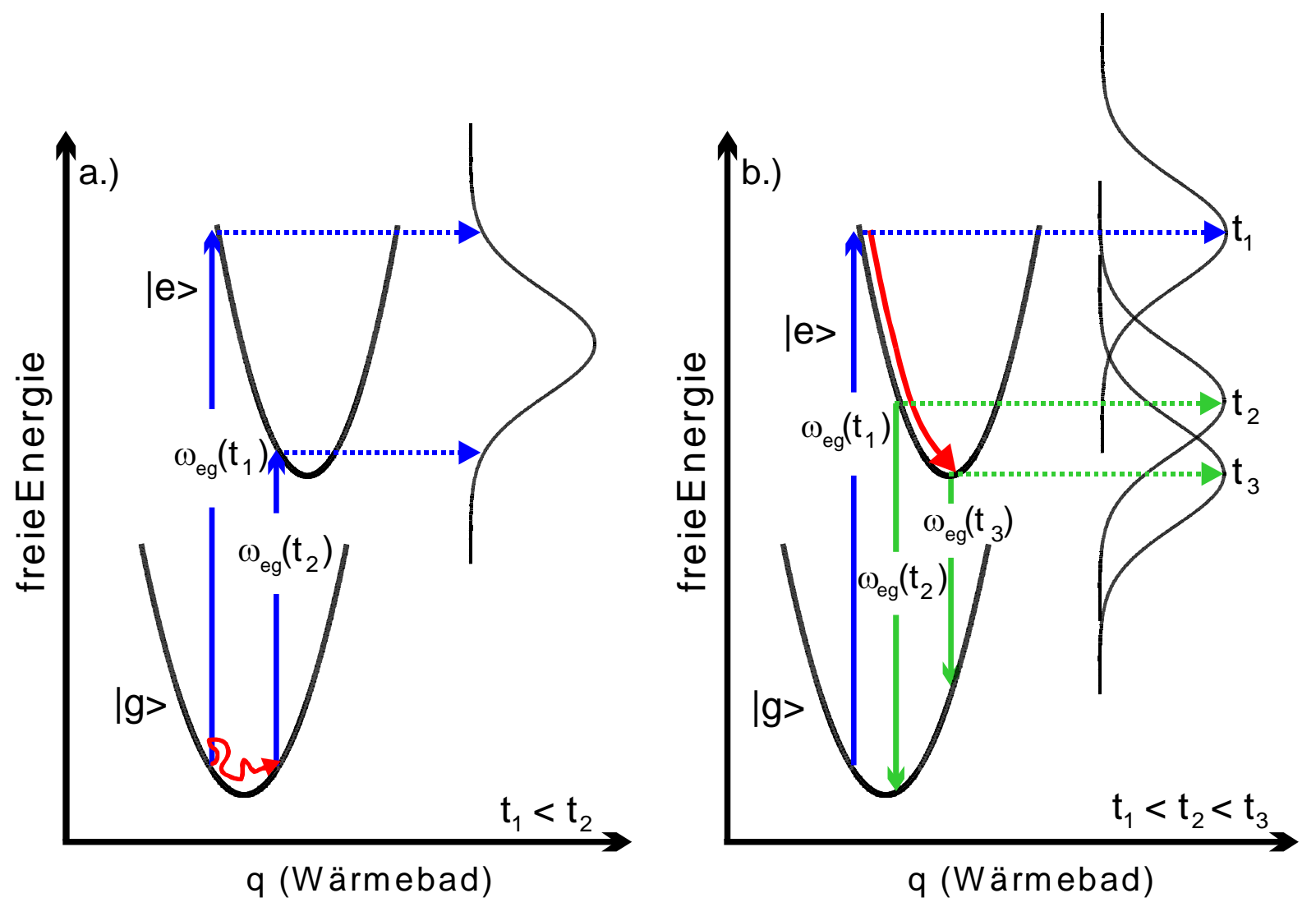

Abbildung 4.1: a.) Thermische Fluktuationen (rot), die eine Verbreiterung des linearen Absorptionsspektrums bewirken;

b.) Solvensrelaxation (dynamische Solvatation) nach optischer Präparation von $|e\rangle\langle e|$.

Ein einfaches Beispiel verdeutlicht die Natur der verallgemeinerten Kernkoordinaten des Bades. Dreht die optische Anregung das Dipolmoment des Zwei-NiveauSystems um $180^{\circ}$ und besteht das Bad aus dipolaren Molekülen, so ist die primäre Komponente von q die Gesamtheit aller Winkel der individuellen Baddipole zum Dipolmoment des Systems im Zustand $|e\rangle$.

Die dynamische Minimierung der System-Bad-Wechselwirkungsenergie durch Variation der verallgemeinerten Badmoden q wird dynamische Solvatation oder auch Solvensreorganisierung genannt (roter Pfeil in Abbildung $4.1 \mathrm{~b}$.)). Sie hat eine zeitabhängige Reduzierung der Energie im angeregten Zustand $|e\rangle$ zur Folge und äußert sich daher als dynamische Rotverschiebung („Stokes-Verschiebung“) des Emissionsspektrums nach gepulster elektronischer Anregung $(|\mathrm{e}\rangle \rightarrow|\mathrm{g}\rangle)$.

Dynamische Stokes-Verschiebung der Fluoreszenz und Verbreiterung der linearen Absorption sind im Grenzfall einer kleinen Störung der Ladungsdichteverteilung beim Übergang $(|\mathrm{e}\rangle \rightarrow|\mathrm{g}\rangle)$ nicht unabhängig voneinander. Die lineare Antworttheorie 
verlangt, dass die Zeitskala für thermische Fluktuationen im Gleichgewicht auch die Zeitskala definiert, mit der das Nichtgleichgewichtssystem relaxiert. Dies bedeutet, dass die System-Bad-Wechselwirkungspotentiale für den Grund- und elektronisch angeregten Zustand identisch sind. Sie unterscheiden sich lediglich in den Gleichgewichtskernlagen der verallgemeinerten Badmoden q. In diesem Fall ist die durch Lösungsmittelumorientierung in $|e\rangle$ freiwerdende Energie (Reorganisationsenergie $\lambda$ ) gleich der halben stationären Stokes-Verschiebung zwischen linearem Absorptions- und Emissionsspektrum. Die Dynamik der Solvatation wird im Allgemeinen durch die Solvatationskorrelationsfunktion quantifiziert:

$$
S(t)=\frac{\tilde{v}(t)-\tilde{v}(\infty)}{\tilde{v}(0)-\tilde{v}(\infty)}
$$

\section{Gleichung 4.1}

wobei $\tilde{v}(\mathrm{t})$ die spektrale Position des zeitabhängigen Emissionsspektrums ist.

Im Grenzfall der linearen Antwort ist diese Größe proportional zur Korrelationsfunktion $M(t)$ der Gleichgewichtsfluktuationen $\delta \omega_{\text {eg }}(t)$ der elektronischen Energielücke:

$$
M(t)=\frac{\left\langle\delta \omega_{e g}(t) \delta \omega_{e g}(0)\right\rangle}{\left\langle\delta \omega_{e g}(0)^{2}\right\rangle}
$$

\section{Gleichung 4.2}

Diese Korrelationsfunktion $\mathrm{M}(\mathrm{t})$ wird auch System-Bad-Korrelationsfunktion genannt, da die Fluktuationen $\delta \omega_{\text {eg }}(\mathrm{t})$ die thermischen Fluktuationen der System-Bad-Wechselwirkungsenergie reflektieren. Letztere resultieren ihrerseits aus thermischen Fluktuationen $\delta q(t)$ der Badkonfiguration.

Mit diesen Überlegungen ergibt sich daher ein ganz anderer Ansatz zum Studium der dynamischen Solvatation, nämlich der Aufklärung von Gleichgewichtsfluktuationen, die zur Verbreiterung des linearen Absorptionsspektrums führen. Präpariert man in dem in Abbildung 4.1 dargestellten Zwei-Niveau-System eine elektronische Kohärenz $|e\rangle\langle g|$, so wird diese Kohärenz aufgrund von Fluktuationen $\delta q(\mathrm{t})$ zeitlich abklingen. Eine solche Kohärenz kann z.B. durch Wechselwirkung des Zwei-Niveau- 
Systems mit einem $\pi / 2$-Puls (analog zur NMR-Spektroskopie) präpariert werden. Die erzeugte Polarisation (1.Ordnung) des makroskopischen Ensembles ist verantwortlich für die Emission des freien Induktionszerfalls (FID). Der FID ist derart gedämpft, dass dessen Fourier-Transformierte dem linearen Absorptionsspektrum entspricht (siehe Prinzip der 1D-FT-NMR). Die Dämpfung des FID bzw. die zeitliche Charakteristik des Kohärenzzerfalls wird auch durch die oben besprochenen Badfluktuationen bestimmt, die die elektronische Energielücke $|e\rangle \rightarrow|g\rangle$ modulieren. Prinzipiell lässt sich allerdings nicht entscheiden, ob der Kohärenzzerfall nicht auch durch eine breite aber statische Verteilung von Übergangsfrequenzen im Ensemble verursacht wird.

Die spektrale Lage $\omega_{\text {eg }}$ der elektronischen Resonanz des i-ten Chromophors im Ensemble lässt sich also zunächst prinzipiell zerlegen gemäß:

$$
\omega_{\mathrm{eg}}^{\mathrm{i}}(\mathrm{t})=\left\langle\omega_{\mathrm{eg}}^{\mathrm{i}}\right\rangle+\varepsilon_{\mathrm{i}}+\delta \omega_{\mathrm{i}}(\mathrm{t})
$$

\section{Gleichung 4.3}

Dabei ist $\left\langle\omega_{\text {eg }}^{i}>\right.$ der Trajektorienmittelwert der Übergangsfrequenz, $\varepsilon_{i}$ ein statischer oder „inhomogener“ Beitrag und $\delta \omega_{\mathrm{i}}(\mathrm{t})$ ein fluktuierender Term, der durch Badfluktuationen $\delta q(\mathrm{t})$ verursacht wird.

Sollen also die badinduzierten Fluktuationen der Übergangsfrequenz $\delta \omega(\mathrm{t})$ ermittelt werden (z.B. in der Form ihrer Autokorrelationsfunktion $M(t)$ ), so gilt es zunächst den statischen Beitrag $\varepsilon$ zu eliminieren. Analog zum Spin-Echo der Kernspinresonanz bieten sich für elektronische Resonanzen Photon-Echo-Techniken an. 


\subsection{Elektronisches 2-Niveau-System in der Bloch'schen Beschreibung}

Wir betrachten zunächst das 2-Niveau System aus Abbildung 4.1 unter dem Einfluss eines elektromagnetischen Feldes $\mathrm{E}(\mathrm{t})$. Der Hamiltonoperator unter Vernachlässigung einer Kopplung an das Bad ist gegeben als:

$$
\hat{\mathrm{H}}(\mathrm{t})=\hat{\mathrm{H}}_{0}+\hat{\mu} \overrightarrow{\mathrm{E}}(\mathrm{t})
$$

\section{Gleichung 4.4}

wobei $\overrightarrow{\mathrm{E}}(\mathrm{t})=\overrightarrow{\mathrm{E}}_{0}(\mathrm{t}) \mathrm{e}^{\mathrm{i} \Omega \mathrm{t}}+$ c.c. und $\hat{\mu}$ der Übergangsdipoloperator ist. Der ungestörte Hamiltonoperator des 2-Niveau-Systems lautet:

$$
\hat{\mathrm{H}}_{0}=\frac{1}{2} \hbar \omega_{\mathrm{eg}}\left(\begin{array}{ll}
1 & 0 \\
0 & 1
\end{array}\right) .
$$

\section{Gleichung 4.5}

Die zeitliche Entwicklung der Dichtematrix $\rho$ wird beschrieben durch die Liouville-van Neumann Gleichung

$$
\dot{\rho}=-\frac{\mathrm{i}}{\hbar}[\hat{\mathrm{H}}, \rho] \text {. }
$$

\section{Gleichung 4.6}

Die Dichtematrix in der Wechselwirkungs-Darstellung (im sogenannten Bloch'schen Rahmen, der mit der Frequenz $\omega_{\text {eg }}$ des eingestrahlten elektromagnetischen Feldes rotiert) hat die Form

$$
\tilde{\rho}=\left(\begin{array}{cc}
\rho_{11} & \rho_{12} \mathrm{e}^{-\mathrm{i} \Omega \mathrm{t} / 2} \\
\rho_{21} \mathrm{e}^{\mathrm{i} \Omega \mathrm{t} / 2} & \rho_{22}
\end{array}\right) \text {. }
$$


In dieser Darstellung verschwinden alle mit $\Omega$ oszillierenden Nicht-Diagonalelemente des Hamilton-Operators:

$$
\tilde{\mathrm{H}}=\left(\begin{array}{cc}
\frac{1}{2} \hbar \Delta & -\mu_{12} \mathrm{E}_{0} \\
-\mu_{21} \mathrm{E}_{0} & \frac{1}{2} \hbar \Delta
\end{array}\right) .
$$

\section{Gleichung 4.8}

Die Größe $\Delta$ entspricht der Laserverstimmung bezüglich der elektronischen Energielücke $\Delta=\Omega-\omega_{\text {eg }}$.

Die Kopplung des elektronischen 2-Niveau-Systems an das Wärmebad wird auf rein phänomenologische Weise mittels eines Relaxationsoperators $\hat{\Gamma}$ eingeführt:

$$
\hat{\mathrm{H}}_{\text {total }}(\mathrm{t})=\hat{\mathrm{H}}(\mathrm{t})+\hat{\Gamma} \quad \text { bzw. } \quad \tilde{\mathrm{H}}_{\text {total }}(\mathrm{t})=\tilde{\mathrm{H}}(\mathrm{t})+\hat{\Gamma} \text {, }
$$

wobei $\hat{\Gamma}$ gegeben ist durch

$$
\hat{\Gamma}=-\frac{1}{2}\left(\begin{array}{cc}
\frac{1}{\mathrm{~T}_{1}} & \frac{1}{\mathrm{~T}_{2}} \\
\frac{1}{\mathrm{~T}_{2}} & \frac{1}{\mathrm{~T}_{1}}
\end{array}\right) .
$$

\section{Gleichung 4.9}

Die Diagonalelemente der Dichtematrix relaxieren mit der Populationsrelaxationszeit $\mathrm{T}_{1}$, während Kohärenzen (d.h. Nichtdiagonaleinträge in $\tilde{\rho}$ ) mit der Phasenrelaxationszeit $\mathrm{T}_{2}$ zerfallen.

Damit wird ein Satz gekoppelter Differentialgleichungen erhalten, der die zeitliche Entwicklung der 2-Niveau-Dichtematrix unter dem gleichzeitigen der Einfluss des elektromagnetischen Feldes und des Bades in der Wechselwirkungs-Darstellung beschreibt: 


$$
\dot{\tilde{\rho}}=\left(\begin{array}{cc}
\mathrm{i} \chi\left(\tilde{\rho}_{21}-\tilde{\rho}_{12}\right)-\frac{1}{\mathrm{~T}} \tilde{\rho}_{11} & -\mathrm{i} \chi\left(\tilde{\rho}_{22}-\tilde{\rho}_{11}\right)-\left(\frac{1}{\mathrm{~T}_{2}}-\mathrm{i} \Delta\right) \tilde{\rho}_{21} \\
\mathrm{i} \chi\left(\tilde{\rho}_{22}-\tilde{\rho}_{11}\right)-\left(\frac{1}{\mathrm{~T}_{2}}+\mathrm{i} \Delta\right) \tilde{\rho}_{12} & -\mathrm{i} \chi\left(\tilde{\rho}_{21}-\tilde{\rho}_{12}\right)-\frac{1}{\mathrm{~T}_{1}} \tilde{\rho}_{22}
\end{array}\right) .
$$

\section{Gleichung 4.10}

Diesen Satz von Differentialgleichungen nennt man auch die optischen BlochGleichungen. Die Rabi-Frequenz $\chi$ ist definiert als

$$
\chi=\frac{\mu_{12} \mathrm{E}_{0}(\mathrm{t})}{\hbar} .
$$

\section{Gleichung 4.11}

Für Gleichung 4.10 existieren keine analytischen Lösungen. Diese sind aber erhältlich für zeitlich invariante Felder $\mathrm{E}_{0}(\mathrm{t})=\mathrm{E}$. Die formale Lösung lautet:

$$
\tilde{\rho}(\mathrm{t})=\exp (\mathrm{i} \mathscr{L} \mathrm{t}) \rho(0)
$$

wobei 2 der zeitunabhängige Liouville-Operator ist. Der Zeitentwicklungsoperator $\exp (\mathrm{i} \mathscr{L}$ t) wird auch Green'sche Funktion genannt. Wir betrachten zunächst den FID nach Einstrahlung eines $\pi / 2-P u l s e s$, d.h.:

$$
\chi \cdot \mathrm{t}=\pi / 2
$$

\section{Gleichung 4.12}

Zur Vereinfachung werden während Einstrahlung des Feldes Relaxationsphänomene vernachlässigt, so dass $1 / T_{2}=1 / T_{1}=0$ ist. Weiterhin sei die Verstimmung innerhalb der Pulsbandbreite vernachlässigbar. Anschließend wird das Feld abgeschaltet und die Dichtematrix propagiert für die Dauer $t$ frei. Dies geschieht allerdings unter Berücksichtigung von Relaxation. Für $\tilde{\rho}(\mathrm{t})$ wird erhalten: 


$$
\tilde{\rho}(\mathrm{t})=\left(\begin{array}{cc}
1-\mathrm{e}^{-\mathrm{t} / \mathrm{T}_{1}} & -\mathrm{ie}^{\left(\mathrm{i} \Delta-1 / \mathrm{T}_{2}\right) \mathrm{t}} \\
\mathrm{ie} \mathrm{e}^{-\left(\mathrm{i} \Delta+1 / \mathrm{T}_{2}\right) \mathrm{t}} & \mathrm{e}^{-\mathrm{t} / \mathrm{T}_{1}}
\end{array}\right) .
$$

\section{Gleichung 4.13}

Damit ergibt sich die feldinduzierte makroskopische Polarisation im Ensemble gemäß

$$
\mathrm{P}(\mathrm{t}, \Delta)=\mathrm{N} \cdot \operatorname{Tr}\left[\mu_{12} \tilde{\rho}(\mathrm{t}, \Delta)\right]
$$

bzw.

$$
\mathrm{P}(\mathrm{t}, \Delta)=\mathrm{N} \cdot \mu_{12} \sin \{(\Omega+\Delta) \mathrm{t}\} \exp \left(-\frac{\mathrm{t}}{\mathrm{T}_{2}}\right) .
$$

\section{Gleichung 4.14}

Die Verstimmung $\Delta$, die im Vergleich zur Pulsbandbreite vernachlässigbar war, spielt bei der freien Propagation eine entscheidende Rolle. Sie repräsentiert den statischen oder inhomogenen Beitrag zur zeitabhängigen elektronischen Energielücke gemäß Gleichung 4.3. Im allgemeinen wird eine Normalverteilung von elektronischen Bohrfrequenzen angenommen:

$$
\mathrm{W}(\Delta)=\frac{\sigma}{\sqrt{2 \pi}} \exp \left(-\frac{\sigma}{2} \Delta^{2}\right)
$$

\section{Gleichung 4.15}

Der Parameter $\sigma$ repräsentiert damit die inhomogene Linienbreite. Die Polarisation muss über diese Verteilung gemittelt werden:

$$
\mathrm{P}(\mathrm{t})=\int_{-\infty}^{\infty} \mathrm{W}(\Delta) \mathrm{P}(\mathrm{t}, \Delta) \mathrm{d} \Delta
$$

\section{Gleichung 4.16}


Damit wird für den FID erhalten:

$$
\mathrm{P}(\mathrm{t})=\mathrm{N} \mu_{12} \frac{\sigma}{\sqrt{2 \pi}} \exp \left(-\frac{\mathrm{t}}{\mathrm{T}_{2}}\right) \exp \left(-\frac{\mathrm{t}^{2}}{2 \sigma}\right)
$$

\section{Gleichung 4.17}

Die Fouriertransformierte des FID liefert das lineare Absorptionsspektrum. Aus Gleichung 4.17 ist ersichtlich, dass anhand der Form des linearen Spektrums keinerlei Information über die badinduzierten Relaxationsphänomene erhalten werden kann.

Wir kommen nun zur 2-Puls-Echo-Sequenz, die analog zum Spin-Echo der Kernresonanzspektroskopie in der Lage ist, die inhomogene Linienbreite zu eliminieren. In diesem Experiment ist das 2-Niveau-System zwei konsekutiver Materie-Feld-Wechselwirkungen unterworfen, die durch eine freie Propagation für die Dauer $\tau$ getrennt sind (siehe Abbildung 4.2).

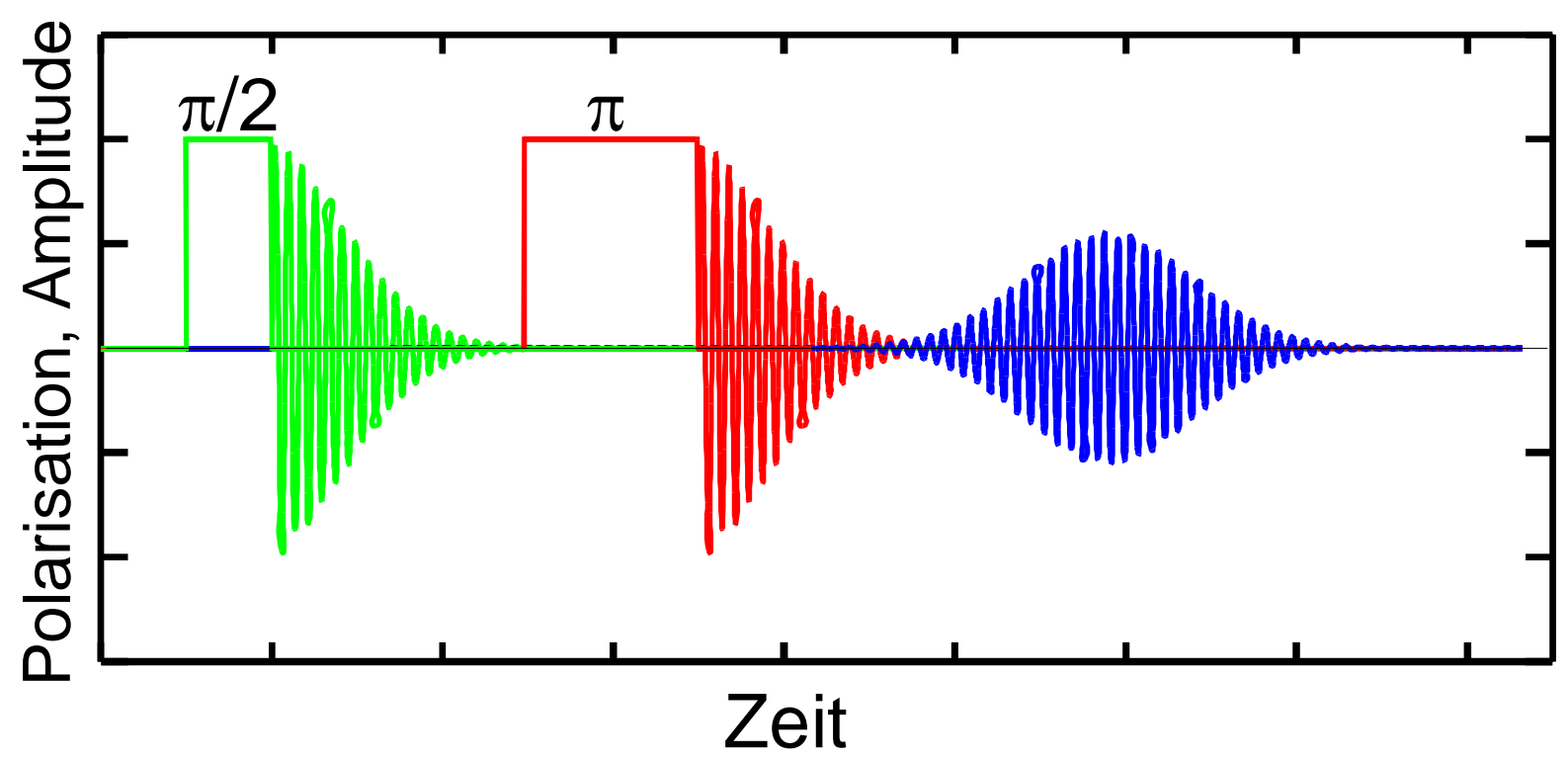

Abbildung 4.2: 2-Puls-Echo-Sequenz mit Polarisationsantwort 
Die aus dieser Pulssequenz resultierende Polarisation muss wieder über die inhomogene Verteilung gemittelt werden:

$$
\mathrm{P}(\mathrm{t}, \Delta)=\int_{-\infty}^{\infty} \mathrm{g}(\Delta) \cdot \mathrm{N} \cdot \operatorname{Tr}\left[\mu_{12} \rho(\mathrm{t}, \tau, \Delta)\right] \mathrm{d} \Delta
$$

\section{Gleichung 4.18}

so dass sich ergibt:

$$
\mathrm{P}(\mathrm{t}, \Delta)=4 \mathrm{~N} \mu_{12} \sin (\Omega \mathrm{t}) \exp \left(-\frac{\mathrm{t}+\tau}{\mathrm{T}_{2}}\right) \exp \left(-\left(\frac{\mathrm{t}-\tau}{\sigma}\right)^{2}\right)
$$

\section{Gleichung 4.19}

Die Polarisation ist maximal, wenn $\mathrm{dP}(\mathrm{t}, \tau) / \mathrm{dt}=0$, d.h.

$$
\mathrm{t}_{\max }=\tau-\frac{\sigma}{\mathrm{T}_{2}}
$$

\section{Gleichung 4.20}

Ist die Resonanz durch inhomogene Verbreiterung dominiert, dann ist $\sigma \rightarrow 0$. Zur Zeit $t_{\max }=\tau$ ist die Polarisation maximal. Das Ensemble emittiert also das sogenannte Photon-Echo. Verschwinden dagegen inhomogene Beiträge zur Resonanz, dann ist $\sigma \rightarrow \infty$. Die Polarisation kling nach Einstrahlung des $\pi$-Pulses einfach exponentiell ab. Ein Echo existiert nicht. Im 2-Puls-Echo-Experiment misst ein Photodetektor wie gewöhnlich das zeitlich integrierte Betragsquadrat der Polarisation:

$$
S(\tau)=\int_{-\infty}^{\infty}|P(t, \tau)|^{2} d t
$$

\section{Gleichung 4.21}


Es wird somit folgender Ausruck erhalten:

$$
S(\tau)=\sqrt{4 \pi} N \mu_{12}^{2} \sigma \exp \left\{\left(\frac{\sigma}{T_{2}}\right)^{2}\right\} \exp \left(-\frac{4}{T_{2}} \cdot \tau\right)
$$

Gleichung 4.22

Die Messung der Photon-Echo-Intensität als Funktion der relativen Verzögerung zwischen $\pi / 2$-Puls und $\pi$-Puls liefert damit die Phasenrelaxationszeit $T_{2}$.

Es werden damit Informationen bezüglich badinduzierter Fluktuationen der elektrischen Energielücke zwischen dem elektronischen Grund- und angeregten Zustand in Form einer phänomenologischen Zeitkonstanten erhalten. Die obigen Bloch-Beschreibungen finden erfolgreiche Anwendung in Systemen, in denen eine deutliche Zeitskalentrennung anzutreffen ist. So ist in Gasen der homogene Beitrag zur Resonanzlinienbreite auf impulsive, unkorrelierte Stöße des 2-Niveau-Systems mit Badteilchen zurückzuführen, während die Dopplerverbreiterung solcher Resonanzen einen inhomogenen Beitrag liefert, den das Photon-Echo eliminieren kann. In Festkörpern werden homogene Linienbreiten aufgrund von ElektronPhonon-Kopplung beobachtet, während Platzunordnungen rein statisch und daher in Echo-Experimenten rephasierbar, d.h. eliminierbar sind. In fluiden Medien ist hingegen eine strikte Zeitskalentrennung nur selten anzutreffen und eine phänomenologische Beschreibung von Phasenrelaxationsprozessen wird unzulässig. An dieser Stelle muss zunächst auf stochastische Modelle zur Resonanzbreite zurückgegriffen und diese in störungstheoretischen Lösungen der Bewegungsgleichung für die Dichtematrix berücksichtigt werden. 


\subsection{Stochastische Theorie zur Resonanzbreite nach Kubo}

In der stochastischen Theorie nach Kubo [67-69] wird die Resonanzlinienform (z.B. die Absorption des Zwei-Niveau-Systems aus Abbildung 4.1) durch stochastische Badfluktuationen bestimmt, die auf variablen Zeitskalen stattfinden können.

Die Bewegungsgleichung für das System in Abbildung 4.1 ist die eines stochastisch gestörten Oszillators:

$$
\dot{\mathrm{q}}(\mathrm{t})=\mathrm{i} \cdot \omega_{\mathrm{eg}}(\mathrm{t}) \cdot \mathrm{q}(\mathrm{t}) .
$$

Gleichung 4.23

Die stochastische Variable $\omega_{\mathrm{eg}}(\mathrm{t})$ ist gegeben durch Gleichung 4.3, so dass mit der Randbedingung $\mathrm{q}(\mathrm{t}=0)=\mathrm{q}_{0}$ die Lösung von Gleichung 4.23 gegeben ist als:

$$
q(t)=q_{0} \exp \left(i \cdot \int_{0}^{t} \omega\left(t^{\prime}\right) d t^{\prime}\right)
$$

Gleichung 4.24

Ist $\omega(\mathrm{t})$ eine stochastische Variable, dann ist $\mathrm{q}(\mathrm{t})$ ein stochastischer Prozess, dessen Korrelationsfunktion geschrieben werden kann als:

$$
\langle q(0) q(t)\rangle=\left\langle\left|q_{0}\right|^{2} \exp \left(i \int_{0}^{t} \omega\left(t^{\prime}\right) d t^{\prime}\right)\right\rangle .
$$

Gleichung 4.25

Unter Berücksichtigung von Gleichung 4.3 ergibt sich

$$
\langle q(0) q(t)\rangle=\exp \left(i \omega_{0} \cdot t\right) \phi(t),
$$


wobei $\omega_{0}=<\omega_{\text {eg }}>$ und

$$
\phi(t)=\left\langle\exp \left(-\mathrm{i} \int_{0}^{\mathrm{t}} \delta \omega\left(\mathrm{t}^{\prime}\right) \mathrm{d \textrm {t } ^ { \prime }}\right)\right\rangle
$$

ist.

Der Prozess $\delta \omega(\mathrm{t})$ ist stationär mit einem Mittelwert $<\delta \omega(\mathrm{t})>=0$. Seine Korrelationsfunktion lautet:

$$
\langle\delta \omega(0) \delta \omega(\mathrm{t})\rangle=\left\langle\delta \omega^{2}\right\rangle \Psi(\mathrm{t})
$$

\section{Gleichung 4.27}

Dabei ist $\left\langle\delta \omega^{2}\right\rangle$ das mittlere Schwankungsquadrat der Fluktuationen der elektronischen Energielücke.

Handelt es sich darüber hinaus um einen Gauß'schen Prozess (d.h. die Wahrscheinlichkeitsdichte für $\mathrm{n}$ unabhängige Stichproben von $\delta \omega(\mathrm{t})$ entlang der Trajektorie entspricht einer Normalverteilung (siehe Abbildung 4.3 b.)), dann kann $\phi(t)$ aus der Korrelationsfunktion der Energielückenfluktuation berechnet werden gemäß [69]:

$$
\phi(\mathrm{t})=\left\langle\exp \left(\mathrm{i} \int_{0}^{\mathrm{t}} \delta \omega(\tau) \mathrm{d} \tau\right)\right\rangle=\exp \left\{-\frac{1}{2} \int_{0}^{\mathrm{t}} \mathrm{d} \tau_{1} \int_{0}^{\mathrm{t}} \mathrm{d} \tau_{2}\left\langle\delta \omega\left(\tau_{1}\right) \delta \omega\left(\tau_{2}\right)\right\rangle\right\}
$$

\section{Gleichung 4.28}

Die Integration von $\tau_{1}$ und $\tau_{2}$ über das Quadrat wird in die Integration über das Dreieck, der Hälfte des Quadrats, geändert. Es ergibt sich:

$$
\phi(t)=\exp \left\{-\int_{0}^{\mathrm{t}} \mathrm{d} \tau_{1} \int_{0}^{\tau_{1}} \mathrm{~d} \tau_{2}\left\langle\delta \omega\left(\tau_{1}\right) \delta \omega\left(\tau_{2}\right)\right\rangle\right\}
$$

\section{Gleichung 4.29}


Eine Änderung der Integrationsvariablen und eine anschließende Integration liefert unter der Annahme von Stationarität folgendes Integral [69]:

$$
\phi(t)=\exp \left\{-\int_{0}^{\mathrm{t}} \mathrm{d} \tau(\mathrm{t}-\tau)\langle\delta \omega(0) \delta \omega(\tau)\rangle\right\} .
$$

\section{Gleichung 4.30}

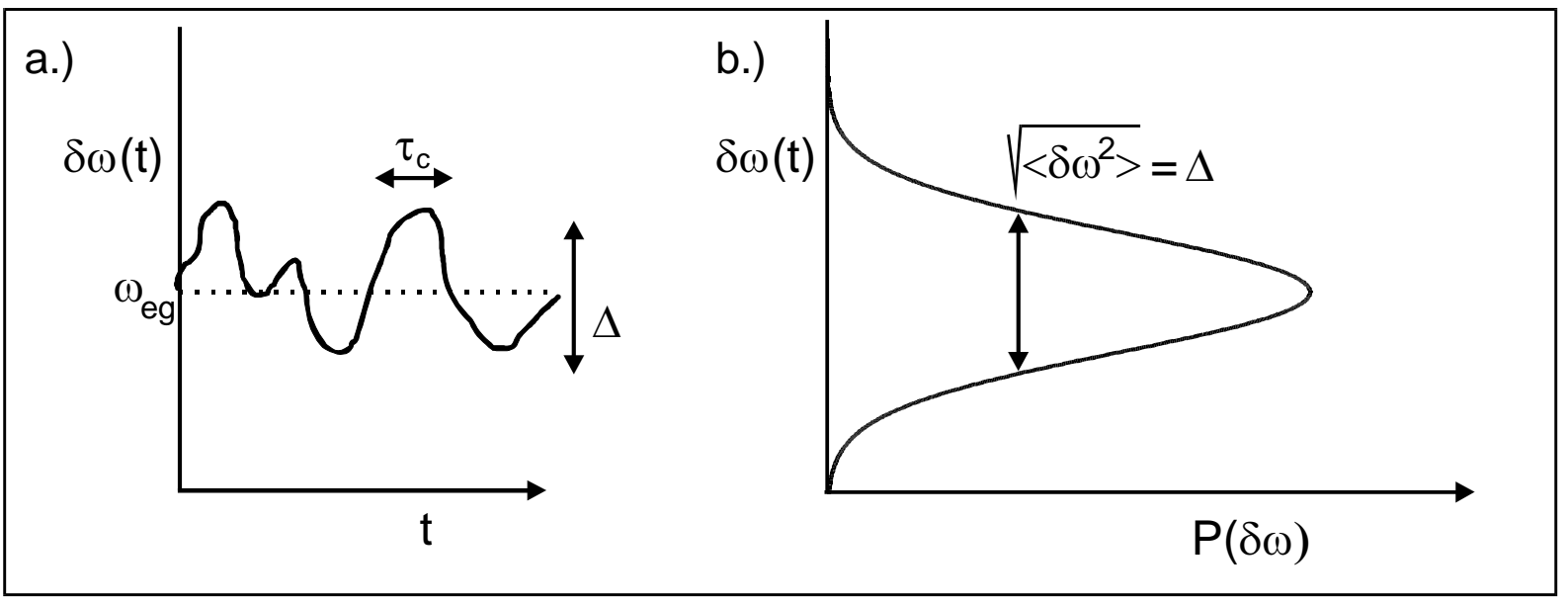

Abbildung 4.3: Trajektorie der Energiefluktuationen eines Chromophors (a.) und zugehörige Wahrscheinlichkeitsverteilung, wenn der stochastische Prozess $\delta \omega(t)$ ein Gauß'scher Prozess ist (b.).

Unter Voraussetzung einer Markov-Modulation wird die Korrelationsfunktion nach Doob's Theorem [78, 79] durch eine Exponentialfunktion beschrieben:

$$
\langle\delta \omega(0) \delta \omega(\tau)\rangle=\Delta^{2} \exp \left(-\frac{\tau}{\tau_{\mathrm{c}}}\right)
$$

\section{Gleichung 4.31}

wobei $\Delta^{2}=<\delta \omega^{2}>$ ist und die Korrelationszeit $\tau_{c}$ definiert wird gemäß:

$$
\tau_{\mathrm{c}}=\frac{1}{\Delta^{2}} \int_{0}^{\infty}\langle\delta \omega(0) \delta \omega(\mathrm{t})\rangle \mathrm{dt} .
$$


Durch Einsetzen von Gleichung 4.31 in Gleichung 4.30 und Integration über $\tau$ ergibt sich folgender Ausdruck für die Korrelationsfunktion $\phi(t)$ :

$$
\phi(\mathrm{t})=\exp \left\{-\Delta^{2} \int_{0}^{\mathrm{t}} \mathrm{d} \tau(\mathrm{t}-\tau) \exp \left(-\frac{\tau}{\tau_{\mathrm{c}}}\right)\right\}=\exp \left(-\left(\Delta \tau_{\mathrm{c}}\right)^{2}\left\{\exp \left(-\frac{\mathrm{t}}{\tau_{\mathrm{c}}}\right)+\frac{\mathrm{t}}{\tau_{\mathrm{c}}}-1\right\}\right)=\exp \left(-\mathrm{g}_{\text {Kubo }}(\mathrm{t})\right)
$$

\section{Gleichung 4.33}

Die Funktion $g_{\text {Kubo }}(\mathrm{t})$ wird auch als Linienform- oder Linienbreitenfunktion bezeichnet [67-69]. Sie liefert durch Fourier-Transformation das Absorptionsprofil:

$$
\mathrm{I}\left(\omega-\omega_{\text {eg }}\right)=\mathrm{FT}(\phi(\mathrm{t}))=\frac{1}{2 \pi} \cdot \int_{-\infty}^{\infty} \exp \left(-\mathrm{i} \cdot\left(\omega-\omega_{\text {eg }}\right) \cdot \mathrm{t}\right) \cdot \phi(\mathrm{t}) \mathrm{dt}
$$

\section{Gleichung 4.34}

Es existieren zwei Grenzfälle, die dem homogenen und inhomogenen Limit der Bloch'schen Beschreibung entsprechen.

Der erste Fall bezieht sich auf vergleichsweise lange Zeiten ( $\left.t \gg \tau_{c}\right)$. Die Linienverbreiterungsfunktion vereinfacht sich unter dieser Vorraussetzung näherungsweise zu:

$$
g(t)=\Delta^{2} \tau_{C} t
$$

\section{Gleichung 4.35}

Die Fouriertransformation ergibt somit eine lorentzförmige Linienform:

$$
\mathrm{I}\left(\omega-\omega_{\mathrm{eg}}\right)=\mathrm{FT}\left(\exp \left(-\Delta^{2} \tau_{\mathrm{C}} \mathrm{t}\right)\right)=\frac{1}{\pi} \cdot \frac{\Delta^{2} \tau_{\mathrm{C}}}{\left(\omega-\omega_{\mathrm{eg}}\right)^{2}+\left(\Delta^{2} \tau_{\mathrm{C}}\right)^{2}}
$$

\section{Gleichung 4.36}


Für kurze Zeiten $\left(\boldsymbol{t} \ll \tau_{c}\right.$ ) verändert sich die Linienverbreiterungsfunktion zu:

$$
g(t)=\frac{\Delta^{2}}{2} t^{2}
$$

\section{Gleichung 4.37}

In diesem Fall ergibt die Fouriertransformation eine gaußförmige Linienform:

$$
\mathrm{I}\left(\omega-\omega_{\mathrm{eg}}\right)=\mathrm{FT}\left(\exp \left(-\frac{\Delta^{2}}{2} \mathrm{t}^{2}\right)\right)=\frac{1}{\sqrt{2 \pi} \Delta} \exp \left(-\frac{\left(\omega-\omega_{\mathrm{eg}}\right)^{2}}{2 \cdot \Delta^{2}}\right) .
$$

\section{Gleichung 4.38}

Diese Näherungen sind jedoch nicht unabhängig von der relativen Geschwindigkeit der Modulationen.

Bei vergleichsweise schnellen Modulationen $\left(\kappa=\Delta \tau_{c}<1\right)$ des Systems durch das Bad ist $\phi(\mathrm{t})$ noch $\approx 1$, wenn $\phi(\mathrm{t})$ den Bereich der Langzeitnäherung erreicht. Daher wird das Abklingverhalten von $\phi(\mathrm{t})$ vom Langzeitgrenzbereich beschrieben und es gilt Gleichung 4.36. Dieser Grenzfall der schnellen Modulationen wird auch „motional narrowing“-Bereich genannt.

Falls die Modulationen des Oszillators durch das Bad vergleichsweise langsam sind ( $\left.\kappa=\Delta \tau_{c}>1\right)$, ist $\phi(\mathrm{t})$ bereits sehr klein, wenn der Langzeitgrenzbereich erreicht wird. Für diesen sogenannten statischen Grenzfall wird daher das Abklingverhalten von $\phi(t)$ vom Kurzeitgrenzbereich wiedergegeben. Es resultiert somit eine gaußförmige Linienform (Gleichung 4.38).

Obwohl der zugrundeliegende Prozess markovisch ist, liefert die stochastische Theorie nach Kubo auch für den Übergang von homogen zu inhomogen in Abhängigkeit von $\Delta \cdot \tau_{c}$ eine Beschreibung der Linienform. 


\subsection{Störungstheoretische Beschreibung von Vier- Wellen-Mischen (4WM)}

Kubo's Modell des stochastisch modulierten Oszillators kann genutzt werden, um im Rahmen störungstheoretischer Lösungen der Liouville-Gleichung, die optische Antwort des Systems zu berechnen. Wir entwickeln zunächst die Dichtematrix

$$
\widetilde{\rho}=\widetilde{\rho}^{(0)}+\widetilde{\rho}^{(1)}+\widetilde{\rho}^{(2)}+\widetilde{\rho}^{(3)}+\ldots . .
$$

Gleichung 4.39

Die individuellen störungsrechnerischen Ordnungen zu $\tilde{\rho}^{(3)}$ sind gemäß $\mathrm{P}^{(\mathrm{i})}=\mathrm{N} \cdot \operatorname{Tr}\left[\mu \tilde{\rho}^{(\mathrm{i})}\right]$ Quellterme für eine makroskopische Polarisation i-ter Ordnung im elektrischen Feld und sind verantwortlich für den FID $(i=1)$, für Frequenzverdopplung und Frequenzmischen in optisch anisotropen Materialien $(i=2)$ bzw. 4-Wellen-Mischen $(i=3)$ wie Photon-Echo-, Pump-Probe- oder transiente GitterSpektroskopie. Zur ersten Ordnung im E-Feld erhalten wir für die zeitabhängige Dichtematrix aus

$$
\dot{\tilde{\rho}}^{(0)}(\mathrm{t})=-\frac{\mathrm{i}}{\hbar}\left[\widetilde{\mathrm{H}}_{\text {total }}(\mathrm{t}), \tilde{\rho}^{(0)}(\mathrm{t})\right]
$$

\section{Gleichung 4.40}

den Ausdruck

$$
\tilde{\rho}^{(1)}\left(\mathrm{t}_{2}\right)=-\frac{\mathrm{i}}{\hbar} \int_{-\infty}^{\mathrm{t}_{2}}\left[\tilde{\mathrm{H}}_{\text {total }}\left(\mathrm{t}_{3}\right), \tilde{\rho}^{(0)}(\mathrm{t}=-\infty)\right] \mathrm{dt}_{3} .
$$


Ganz analog wird aus

$$
\dot{\tilde{\rho}}^{(3)}(\mathrm{t})=-\frac{\mathrm{i}}{\hbar}\left[\widetilde{\mathrm{H}}_{\text {total }}(\mathrm{t}), \tilde{\rho}^{(3)}(\mathrm{t})\right]
$$

\section{Gleichung 4.42}

das Ergebnis

$$
\tilde{\rho}^{(3)}(\mathrm{t})=\left(-\frac{\mathrm{i}}{\hbar}\right)^{3} \int_{-\infty}^{\mathrm{t}} \int_{-\infty}^{\mathrm{t}_{1}} \int_{-\infty}^{\mathrm{t}_{2}} \mathrm{dt}_{3} \mathrm{dt}_{2} \mathrm{dt}_{1}\left[\widetilde{\mathrm{H}}_{\text {total }}\left(\mathrm{t}_{1}\right),\left[\widetilde{\mathrm{H}}_{\text {total }}\left(\mathrm{t}_{2}\right),\left[\widetilde{\mathrm{H}}_{\text {total }}\left(\mathrm{t}_{3}\right), \tilde{\rho}^{(0)}(\mathrm{t}=-\infty)\right]\right]\right.
$$

\section{Gleichung 4.43}

erhalten, wobei $t>t_{1}>t_{2}>t_{3}$ ist.

Mukamel und Mitarbeitern folgend, lässt sich die Polarisation 3.Ordnung darstellen gemäß:

$\mathrm{P}^{(3)}\left(\mathrm{t}, \mathrm{t}_{12}, \mathrm{t}_{23}\right) \propto$

$\left.\begin{array}{rl}\left(\frac{\mathrm{i}}{\hbar}\right)^{3} \exp \left(-\frac{\mathrm{t}_{23}}{\mathrm{~T}_{1}}\right)_{0}^{\infty} \int_{0} \mathrm{dt}_{3} \int_{0}^{\infty} \mathrm{dt}_{2} \int_{0}^{\infty} \mathrm{dt}_{1}\left\{\begin{array}{l}{\left[\mathrm{R}_{2}+\mathrm{R}_{3}\right] \times} \\ \mathrm{E}_{3}\left(\mathrm{t}-\mathrm{t}_{3}\right) \mathrm{E}_{2}\left(\mathrm{t}+\mathrm{t}_{23}-\mathrm{t}_{3}-\mathrm{t}_{2}\right) \mathrm{E}_{1}^{*}\left(\mathrm{t}+\mathrm{t}_{12}+\mathrm{t}_{23}-\mathrm{t}_{3}-\mathrm{t}_{2}-\mathrm{t}_{1}\right) \\ \exp \left[-\mathrm{i}\left(\omega_{\mathrm{eg}}-\Omega\right)\left(\mathrm{t}_{3}-\mathrm{t}_{1}\right)\right] \\ \exp \left[-\mathrm{i}\left(\mathrm{k}_{3}+\mathrm{k}_{2}-\mathrm{k}_{1}\right) \cdot \mathrm{r}\right]+ \\ {\left[\mathrm{R}_{1}+\mathrm{R}_{4}\right] \times} \\ \mathrm{E}_{3}\left(\mathrm{t}-\mathrm{t}_{3}\right) \mathrm{E}_{2}\left(\mathrm{t}+\mathrm{t}_{23}-\mathrm{t}_{3}-\mathrm{t}_{2}-\mathrm{t}_{1}\right) \mathrm{E}_{1}^{*}\left(\mathrm{t}+\mathrm{t}_{12}+\mathrm{t}_{23}-\mathrm{t}_{3}-\mathrm{t}_{2}\right) \\ \exp \left[-\mathrm{i}\left(\omega_{\mathrm{eg}}-\Omega\right)\left(\mathrm{t}_{3}+\mathrm{t}_{1}\right)\right] \\ \exp \left[-\mathrm{i}\left(\mathrm{k}_{3}+\mathrm{k}_{2}-\mathrm{k}_{1}\right) \cdot \mathrm{r}\right]\end{array}\right. \\ \left(\frac{\mathrm{i}}{\hbar}\right)^{3} \exp \left(-\frac{\mathrm{t}_{23}}{\mathrm{~T}_{1}}\right)_{0}^{\infty} \int_{0} \mathrm{dt}_{3} \int_{0}^{\infty} \mathrm{dt}_{2} \int_{0}^{\infty} \mathrm{dt} \mathrm{t}_{1}\left\{\begin{array}{l}{\left[\mathrm{R}_{2}+\mathrm{R}_{3}\right] \times} \\ \mathrm{E}_{3}\left(\mathrm{t}+\mathrm{t}_{23}-\mathrm{t}_{3}-\mathrm{t}_{2}\right) \mathrm{E}_{2}\left(\mathrm{t}-\mathrm{t}_{3}\right) \mathrm{E}_{1}^{*}\left(\mathrm{t}+\mathrm{t}_{12}+\mathrm{t}_{23}-\mathrm{t}_{3}-\mathrm{t}_{2}-\mathrm{t}_{1}\right) \\ \exp \left[-\mathrm{i}\left(\omega_{\mathrm{eg}}-\Omega\right)\left(\mathrm{t}_{3}-\mathrm{t}_{1}\right)\right] \\ \exp \left[-\mathrm{i}\left(\mathrm{k}_{3}+\mathrm{k}_{2}-\mathrm{k}_{1}\right) \cdot \mathrm{r}\right]+ \\ {\left[\mathrm{R}_{1}+\mathrm{R}_{4}\right] \times} \\ \mathrm{E}_{3}\left(\mathrm{t}+\mathrm{t}_{23}-\mathrm{t}_{3}-\mathrm{t}_{2}-\mathrm{t}_{1}\right) \mathrm{E}_{2}\left(\mathrm{t}-\mathrm{t}_{3}\right) \mathrm{E}_{1}^{*}\left(\mathrm{t}+\mathrm{t}_{12}+\mathrm{t}_{23}-\mathrm{t}_{3}-\mathrm{t}_{2}\right) \\ \exp \left[-\mathrm{i}\left(\omega_{\mathrm{eg}}-\Omega\right)\left(\mathrm{t}_{3}+\mathrm{t}_{1}\right)\right] \\ \exp \left[-\mathrm{i}\left(\mathrm{k}_{3}+\mathrm{k}_{2}-\mathrm{k}_{1}\right) \cdot \mathrm{r}\right]\end{array}\right.\end{array}\right\}$

\section{Gleichung 4.44}


Die optische Energielücke $\omega_{\text {eg }}$ kann dem 1. Moment des linearen Absorptionsspektrums entnommen werden. Die Größe $\Omega$ ist wiederum die Frequenz der eingestrahlten elektromagnetischen Felder $E_{1}(t)$ bis $E_{3}(t)$ mit den Wellenvektoren $k_{1}$ bis $k_{3}$. Die Terme $R_{i}$ sind die sogenannten nichtlinearen Antwortfunktionen, die für die Emission eines Echos in die Phasenanpassungsrichtung $\mathrm{k}_{3}+\mathrm{k}_{2}-\mathrm{k}_{1}$ verantwortlich sind. Die Antwortfunktionen tragen der korrekten zeitlichen Entwicklung der Dichtematrix Rechnung, die bei der Wechselwirkung mit den drei konsekutiven E-Feldern berücksichtig werden müssen. Diese sind in Abbildung 4.4 als doppelseitige Feynmann-Diagramme illustriert. Unter Ausnutzung einer Kumulanten-Entwicklung [72] lassen sich die nichtlinearen Antwortfunktionen $R_{i}$ aus der Linienbreitenfunktion $\mathrm{g}(\mathrm{t})$ berechnen gemäß:

$$
\begin{aligned}
& \mathrm{R}_{1}\left(\mathrm{t}_{3}, \mathrm{t}_{2}, \mathrm{t}_{1}\right)=\exp \left[-\mathrm{g}^{*}\left(\mathrm{t}_{3}\right)-\mathrm{g}\left(\mathrm{t}_{1}\right)-\mathrm{f}_{+}\left(\mathrm{t}_{3}, \mathrm{t}_{2}, \mathrm{t}_{1}\right)\right], \\
& \mathrm{R}_{2}\left(\mathrm{t}_{3}, \mathrm{t}_{2}, \mathrm{t}_{1}\right)=\exp \left[-\mathrm{g}^{*}\left(\mathrm{t}_{3}\right)-\mathrm{g}^{*}\left(\mathrm{t}_{1}\right)+\mathrm{f}_{+}^{*}\left(\mathrm{t}_{3}, \mathrm{t}_{2}, \mathrm{t}_{1}\right)\right], \\
& \mathrm{R}_{3}\left(\mathrm{t}_{3}, \mathrm{t}_{2}, \mathrm{t}_{1}\right)=\exp \left[-\mathrm{g}\left(\mathrm{t}_{3}\right)-\mathrm{g}^{*}\left(\mathrm{t}_{1}\right)+\mathrm{f}_{-}^{*}\left(\mathrm{t}_{3}, \mathrm{t}_{2}, \mathrm{t}_{1}\right)\right], \\
& \begin{aligned}
\mathrm{R}_{4}\left(\mathrm{t}_{3}, \mathrm{t}_{2}, \mathrm{t}_{1}\right)=\exp \left[-\mathrm{g}\left(\mathrm{t}_{3}\right)-\mathrm{g}\left(\mathrm{t}_{1}\right)-\mathrm{f}_{-}\left(\mathrm{t}_{3}, \mathrm{t}_{2}, \mathrm{t}_{1}\right)\right], \\
\text { mit } \quad \mathrm{f}_{-}\left(\mathrm{t}_{3}, \mathrm{t}_{2}, \mathrm{t}_{1}\right) \equiv \mathrm{g}\left(\mathrm{t}_{2}\right)-\mathrm{g}\left(\mathrm{t}_{2}+\mathrm{t}_{3}\right)-\mathrm{g}\left(\mathrm{t}_{1}+\mathrm{t}_{2}\right)+\mathrm{g}\left(\mathrm{t}_{1}+\mathrm{t}_{2}+\mathrm{t}_{3}\right), \\
\mathrm{ft}_{+}\left(\mathrm{t}_{3}, \mathrm{t}_{2}, \mathrm{t}_{1}\right) \equiv \mathrm{g}^{*}\left(\mathrm{t}_{2}\right)-\mathrm{g}^{*}\left(\mathrm{t}_{2}+\mathrm{t}_{3}\right)-\mathrm{g}\left(\mathrm{t}_{1}+\mathrm{t}_{2}\right)+\mathrm{g}\left(\mathrm{t}_{1}+\mathrm{t}_{2}+\mathrm{t}_{3}\right) .
\end{aligned}
\end{aligned}
$$

\section{Gleichung 4.45}

Die Funktionen $R_{2}$ und $R_{3}$ beschreiben das konventionelle Photon-Echo bei Anwesenheit von inhomogenen Beiträgen zur Linienbreite. Die Funktionen $R_{1}$ und $R_{4}$ entsprechen dem sogenannten virtuellen Echo und liefern Beiträge zur Polarisation 3. Ordnung, die stets zur Zeit $\mathrm{t}=0$ maximal sind (siehe auch FID und PE im homogenen Limit des Bloch-Modells). Wird in Gleichung 4.44 und Gleichung 4.45 die Kubo'sche Linienbreitenfunktion angewendet und wird $k_{3}=k_{2}$ bzw. $t_{3}=t_{2}$ gesetzt, so lässt sich ein 2-Puls-Photon-Echo im Modell des stochastisch modulierten Oszillators numerisch berechnen. 
$\mathrm{R}_{1}$

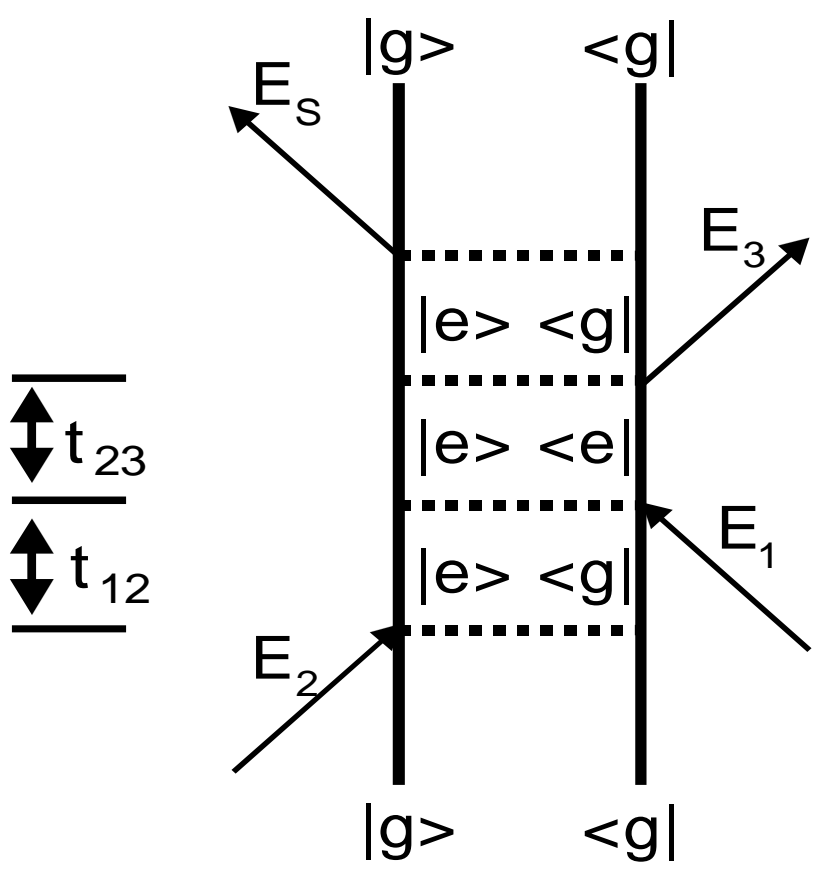

$\mathrm{R}_{3}$

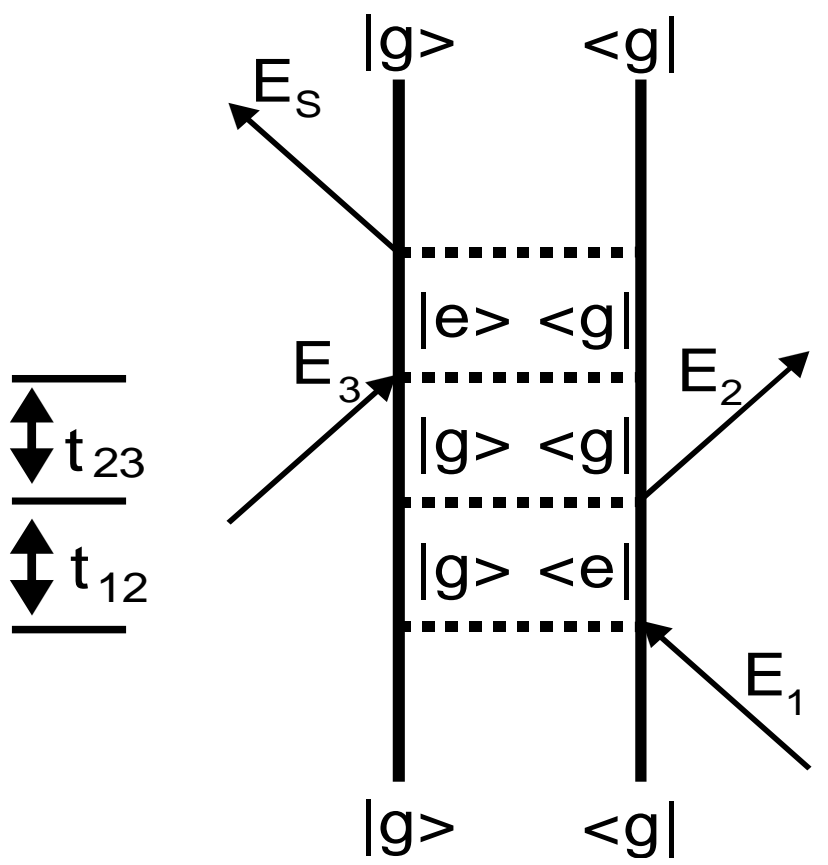

$\mathrm{R}_{2}$

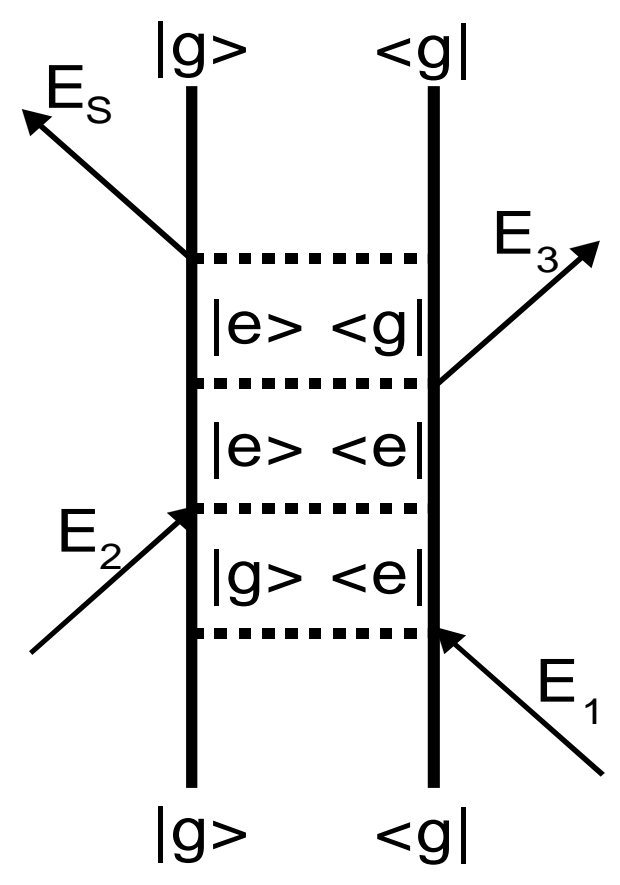

$\mathrm{R}_{4}$

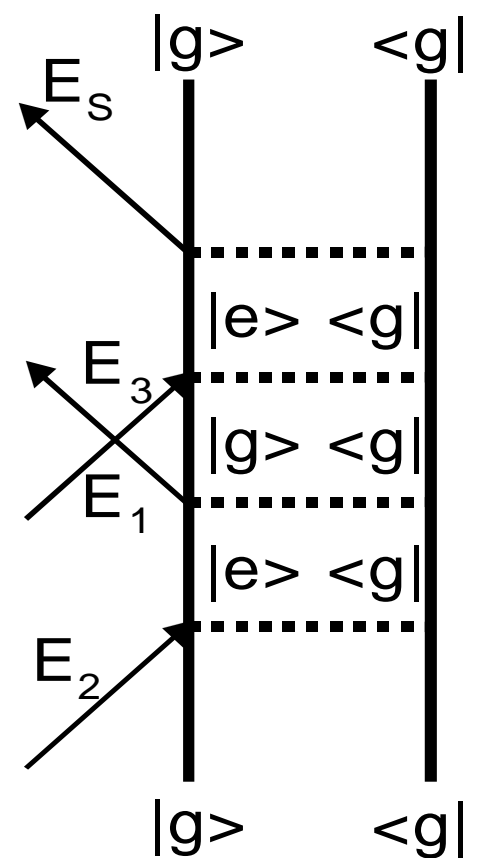

Abbildung 4.4: Feynman-Diagramme zur Beschreibung der nichtlinearen Antwortfunktion 3. Ordnung in Richtung des Wellenvektors $k_{4}=k_{3}+k_{2}-k_{1}$. $R_{1}$ und $R_{4}$ beschreiben die nicht rephasierenden Terme (virtuelles Echo), während $\mathbf{R}_{2}$ und $R_{3}$ die rephasierenden Antwortfunktionen für das reale Echo sind. Die Zeiten $t_{23}$ und $t_{12}$ sind am linken Rand markiert. Ein Pfeil in Richtung des Diagramms bedeutet Energiezufuhr (Absorption), während ein Pfeil weg vom Diagramm Energieabstrahlung (stimulierte Emission) anzeigt. 
Eine Simulation des zeitlich-integrierten 2-Puls-Echos bei Verwendung von gaußförmigen Lichtimpulsen mit einer Dauer von 10 fs sind in Abbildung 4.5 gezeigt. Überraschenderweise lässt sich erkennen, dass im statischen Grenzfall des KuboModells das 2-Puls-Echo nicht rein exponentiell abklingt. Dies ist umso erstaunlicher da die Linienform des Kubomodells ein gaußförmiges Profil vorhersagt, welches dem inhomogenen Grenzfall der Bloch'schen Beschreibung entspricht. Es sei angemerkt, dass die Abweichung vom rein exponentiellen Abklingen nicht auf Faltungseffekte mit den Lichtpulsen zurückzuführen ist.

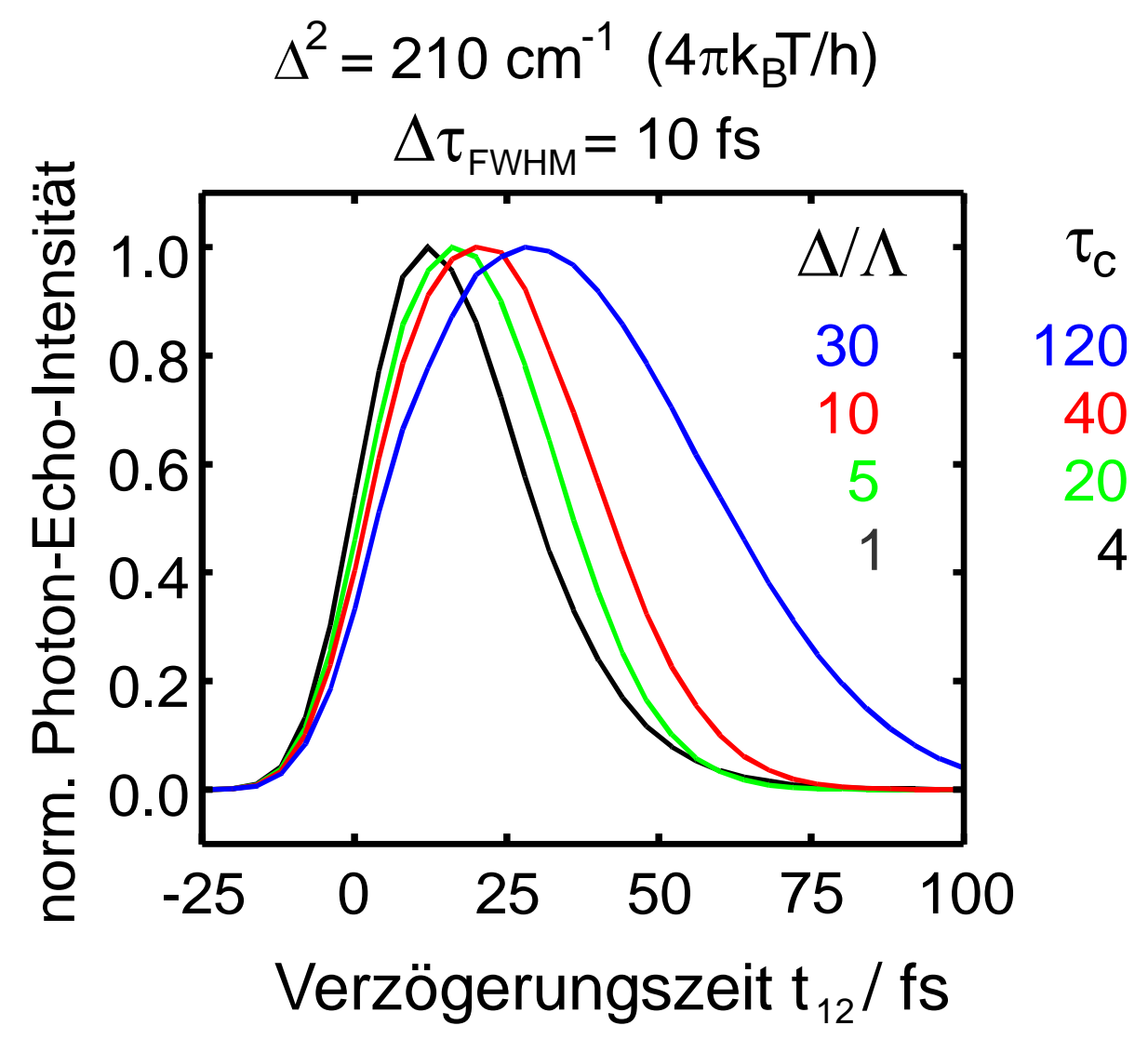

Abbildung 4.5: Berechnete zeitlich integrierte 2-Puls-Echo-Signale

Bemerkenswerterweise ergibt sich, dass beim Übergang vom statischen Grenzfall zum Limit des „motional narrowings“ das Echo sich in Richtung verschwindender Verzögerungszeiten verschiebt. Dieser Effekt wird als „Photon-Echo-Peak-Shift“ bezeichnet. Die zeitliche Verzögerung des Echomaximums (beobachtet im konventionellen, zeitlich-integrierten Echo) ist ein direktes Maß für die Inhomogenität der System-Bad-Wechselwirkungen. Diese Beobachtung liefert die Grundlage für das später zu besprechende 3-Puls-Echo-Peak-Shift-Experiment, mit dem das Abklingen der System-Bad-Korrelationsfunktion quantitativ verfolgt werden kann. 


\subsection{Das Modell der Brown'schen Oszillatoren}

Das Modell der Brown'schen Oszillatoren beruht auf dem Konzept, dass bestimmte Freiheitsgrade des Bades aus der Gesamtheit der Badmoden für die Kopplung an das 2-Niveau-System von herausragender Bedeutung sind. Diese relevanten Badmoden werden wie harmonische Oszillatoren behandelt. Deren Bewegungsgleichung ist gegeben durch eine verallgemeinerte Langevin-Gleichung

$$
\mu \omega^{2} \mathrm{q}(\mathrm{t})+\mu \int_{0}^{\mathrm{t}} \gamma_{\mathrm{j}}(\mathrm{t}-\tau) \cdot \dot{\mathrm{q}}(\tau) \mathrm{d} \tau+\mu \ddot{\mathrm{q}}(\mathrm{t})=\mu \mathrm{F}(\mathrm{t}) .
$$

\section{Gleichung 4.46}

Die verbleibenden Badmoden resultieren in einer frequenzabhängigen Reibung und damit dem Reibungskernel $\hat{\gamma}(\mathrm{t})$, sowie einer stochastischen Kraft $\mathrm{F}(\mathrm{t})$, die von den relevanten Badfreiheitsgraden wahrgenommen wird. Diese beiden badgemittelten Größen sind über das Fluktuations-Dissipations-Theorem miteinander verknüpft. Die Korrelationsfunktion $\mathrm{M}(\mathrm{t})$ der Auslenkung des Brown'schen Oszillators hängt ebenfalls von der frequenzabhängigen Reibung ab und ist über die Laplace-Transformierte der Langevin-Gleichung erhältlich:

$$
M(t)=\mathscr{L}^{-1}\left\{\frac{\mathrm{s}+\gamma(\mathrm{s})}{\mathrm{s}^{2}+\mathrm{s} \gamma(\mathrm{s})+\omega^{2}}\right\},
$$

\section{Gleichung 4.47}

wobei $\gamma(\mathrm{s})=\int_{0}^{\infty} \mathrm{e}^{-\mathrm{st}} \hat{\gamma}(\mathrm{t}) \mathrm{dt}$ und $\mathscr{L}^{-1}$ die inverse Laplace-Transformation ist.

Die Frequenzabhängigkeit der Reibung wird im Allgemeinen vernachlässigt, so dass sich

$$
\gamma(\mathrm{s})=\gamma(\mathrm{s}=0)=\gamma
$$


ergibt. Diese sogenannte Markov'sche Näherung ist zulässig, wenn die stochastische Kraft aus Gleichung 4.46 schneller flukuiert als die Bewegungen des Oszillators.

Die Korrelationsfunktion $M(t)$ kann ihrerseits genutzt werden, um eine zum KuboModell analoge Linienbreitenfunktion $\mathrm{g}(\mathrm{t})$ zu formulieren:

$$
g(t)=\Delta^{2} \int_{0}^{t} d \tau_{1} \int_{0}^{\tau_{1}} d \tau_{2} M^{\prime}\left(\tau_{2}\right)-i \lambda \int_{0}^{t} d \tau_{3}\left[1-M^{\prime \prime}\left(\tau_{3}\right)\right]
$$

\section{Gleichung 4.49}

wobei M'(t) der Realteil und M'”(t) der Imaginärteil von $M(t)$ sind.

Wir betrachten im folgenden zwei Spezialfälle von Brown'schen Oszillatoren:

1.) Untergedämpfte Moden, $\gamma<<2 \omega$

$$
M(t)=\mathscr{L}^{-1}\left\{\frac{\mathrm{s}+\gamma}{\mathrm{s}^{2}+\mathrm{s} \gamma+\omega}\right\} .
$$

\section{Gleichung 4.50}

Daraus ergibt sich

$$
\mathrm{M}(\mathrm{t})=\exp \left(-\frac{\gamma \cdot \mathrm{t}}{2}\right)\left[\cos (\Omega \cdot \mathrm{t})+\frac{\gamma}{2 \Omega} \sin (\Omega \cdot \mathrm{t})\right],
$$

\section{Gleichung 4.51}

mit $\Omega=\sqrt{\omega^{2}+\left(\frac{\gamma}{2}\right)^{2}}$ und $M^{\prime}(t)=M^{\prime \prime}(t) \equiv M(t)$.

Die Korrelationsfunktion entspricht einer gedämpften Schwingung. Im linearen Absorptionsspektrum resultiert sie in einer Feinstruktur. Daher kann dieser Fall genutzt werden, um chromophorspezifische Franck-Condon-Progressionen zu reproduzieren. 
2.) Stark übergedämpfte Moden, $\gamma>>2 \omega$

Im Fall stark übergedämpfter Moden vereinfacht sich die Langevin -Gleichung zu:

$$
\omega^{2} \mathrm{q}(\mathrm{t})+\gamma \cdot \dot{\mathrm{q}}(\tau)=\mathrm{F}(\mathrm{t})
$$

\section{Gleichung 4.52}

Daraus folgt:

$$
M(t)=\mathscr{L}^{-1}\left\{\frac{\gamma}{\omega^{2}+\gamma s}\right\}
$$

\section{Gleichung 4.53}

und somit

$$
M(t)=e^{-\Lambda t}=M^{\prime}(t)=M^{\prime \prime}(t)
$$

\section{Gleichung 4.54}

mit $\Lambda=\omega^{2} / \gamma$. Die Bewegung des Oszillators ist rein diffusiv. Dies liegt an der Vernachlässigung des Beschleunigungsterms $\mu \ddot{q}(\mathrm{t})$. Eine Trägheitsbewegung des Oszillators zu frühen Zeiten existiert nicht. Stark übergedämpfte Moden können genutzt werden, um die für Flüssigkeiten typische Debye-Relaxation als Folge Brown'scher Rotationsdynamik zu simulieren. Einsetzen von Gleichung 4.54 in Gleichung 4.49 liefert für die Linienbreitenfunktion $g(t)$ den stark übergedämpften Oszillator:

$$
\mathrm{g}_{\text {MBO }}(\mathrm{t})=\mathrm{g}_{\text {Kubo }}(\mathrm{t})+\mathrm{i} \cdot \lambda \tau_{\mathrm{c}}\left[1-\exp \left(-\frac{\mathrm{t}}{\tau_{\mathrm{c}}}\right)\right]
$$

\section{Gleichung 4.55}

wobei $\tau_{\mathrm{c}}=\Lambda^{-1}$. Daraus wird ersichtlich, dass das Modell der Brown'schen Oszillatoren für stark übergedämpfte Moden zu Kubo's stochastischer Theorie zur Resonanzbreite äquivalent ist. 


\subsection{Drei-Puls-Echo-Spektroskopie}

Die Drei-Puls-Echo-Spektroskopie unterscheidet sich von der 2-PE-Spektroskopie in einer zusätzlichen Verzögerungszeit $t_{23}$. Es handelt sich daher um eine zweidimensionale Spektroskopie. Den Feynmann-Diagrammen (Abbildung 4.4) entnehmen wir, dass sich das System während der Zeit $t_{23}$ entweder im Grundzustand oder im angeregten Zustand befindet. Aus diesem Grund kann das Drei-Puls-Echo (3-PE) zum Studium von spektraler Diffusion [72, 73] verwendet werden. Die Verzögerung $t_{23}$ definiert dabei die Zeitskala, auf der ein Prozess homogen beziehungsweise inhomogen erscheint. In Abbildung 4.6 ist die Pulssequenz eines 3$P E-E x p e r i m e n t s$ veranschaulicht. Die drei elektromagnetischen Felder sind mit $E_{1}$ bis $E_{3}$ gekennzeichnet. Die erzeugte Polarisation $\mathrm{P}^{(3)}$ ist grau schraffiert dargestellt.

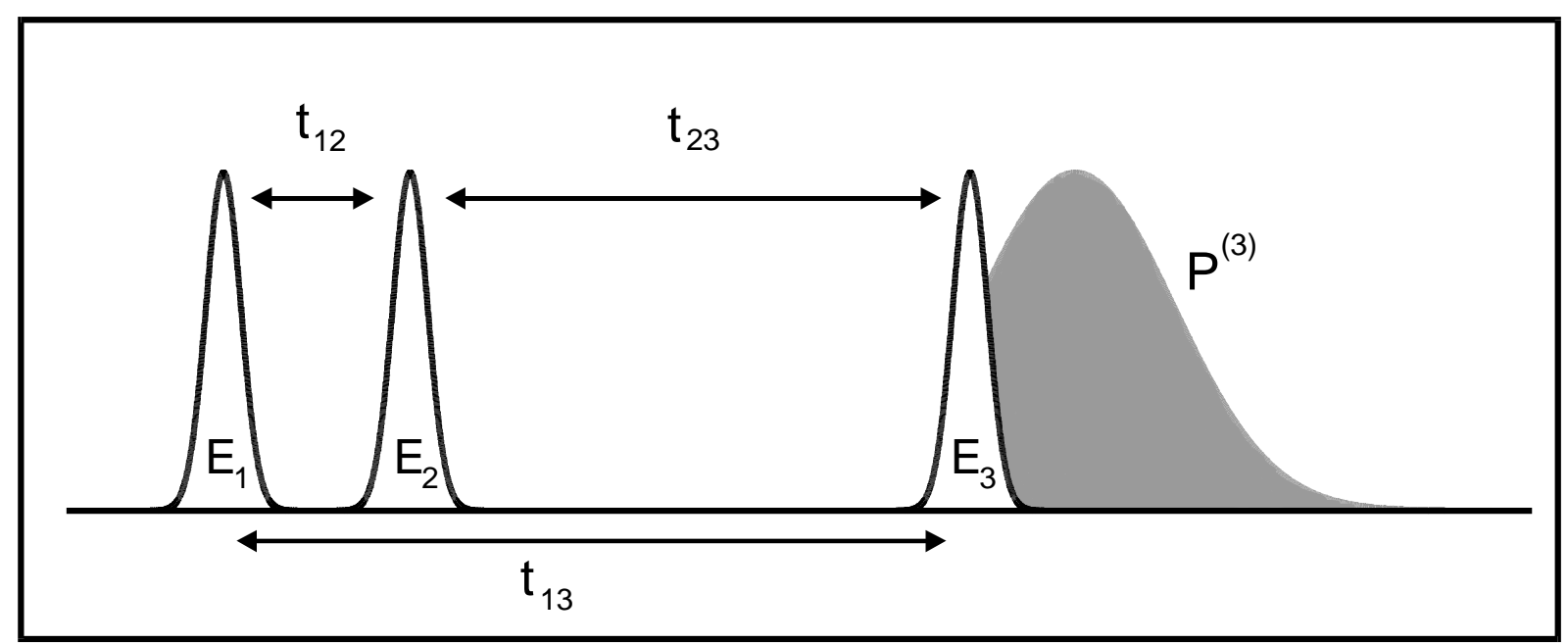

Abbildung 4.6: Dargestellt ist die 3-Puls-Echo-Sequenz und die abgestrahlte Polarisation $\mathrm{P}^{(3)}$. Die 2-Puls-Echo-Sequenz ergibt sich für $t_{23}=0$.

Die Observable des 3-Puls-Echos ist:

$$
I\left(t_{23}, t_{12}\right)=\int_{0}^{\infty}\left|P^{(3)}\left(t, t_{23}, t_{12}\right)\right|^{2} d t .
$$

\section{Gleichung 4.56}

Die Intensität I wird für einen festen Zeitabstand zwischen dem zweiten und dritten Puls ( $t_{23}=$ konstant) in Abhängigkeit von $\mathrm{t}_{12}$ bestimmt. 
In molekularen Flüssigkeiten wird häufig beobachtet, dass die System-BadKorrelationsfunktion auf zwei Zeitskalen abklingt. Der Realteil der Korrelationsfunktion $M(t)$ kann dann in einen schnellen und einen langsamen Anteil zerlegt werden $[74,75]$ :

$$
M^{\prime}(t)=(1-a) \cdot M_{\text {fast }}^{\prime}(t)+a \cdot M_{\text {slow }}^{\prime}(t) . \quad 0 \leq a \leq 1
$$

\section{Gleichung 4.57}

Es ist zu beachten, dass Kubo's stochastische Theorie einen solchen Fall nicht berücksichtigt. Das Modell der Brown'schen Oszillatoren allerdings beinhaltet ein solches Abklingen von $\mathrm{M}(\mathrm{t})$. So ist vorstellbar, dass das Langzeitverhalten von $\mathrm{M}(\mathrm{t})$ durch einen stark übergedämpften Oszillator gekennzeichnet ist, während das Kurzzeitverhalten durch destruktive Interferenz einer Vielzahl von untergedämpften Oszillatoren charakterisiert wird. In diesem Fall kann $\mathrm{g}(\mathrm{t})$ als $\sum \mathrm{g}(\mathrm{t})$ angesetzt werden.

Gleichung 4.57 gilt nur dann, wenn der schnelle Anteil zur Zeit $t_{23}$ gleich Null $\left(M_{\text {fast }}^{\prime}(t)=0\right.$ für $\left.t \geq t_{23}\right)$ ist und $M_{\text {slow }}^{\prime}$ auf Zeitskalen von $t$ und $t_{12}$ stationär bleibt. Mathematische Vereinfachungen führen zu folgendem Zusammenhang [75, 76]:

$$
\mathrm{I}\left(\mathrm{t}_{23}, \mathrm{t}_{12}\right) \propto \exp \left(-\frac{2 \mathrm{t}_{23}}{\mathrm{~T}_{1}}\right) \exp \left[-\Delta^{2} \mathrm{t}_{12}^{2}\left(1-\mathrm{a}^{2} \mathrm{M}_{\text {slow }}^{\prime}\left(\mathrm{t}_{23}\right)\right)\right] \times\left\{\frac{\sqrt{\pi}}{2}+\operatorname{erf}\left[\Delta \cdot \mathrm{a} \cdot \mathrm{M}_{\text {slow }}^{\prime}\left(\mathrm{t}_{23}\right) \cdot \mathrm{t}_{12}\right]\right\} .
$$

\section{Gleichung 4.58}

Die Abkürzung „erf“ steht für die Fehlerfunktion:

$$
\operatorname{erf}(\mathrm{x})=\frac{2}{\sqrt{\pi}} \cdot \int_{0}^{\mathrm{x}} \exp \left(-\mathrm{t}^{2}\right) \mathrm{dt}
$$

\section{Gleichung 4.59}

Es sei betont, dass der Imaginärteil M'”(t) der Korrelationsfunktion innerhalb dieser Näherungen keinen Einfluss auf das Signal hat. In Abbildung 4.5 (Kubo-Modell) wurde gezeigt, dass sich mit zunehmendem $\Delta \tau_{c}$ das Maximum des 2-Puls-Echos zu 
größeren Verzögerungszeiten verschiebt. Es stellt sich die Frage, ob mit Hilfe der 3Puls-Echo-Spektroskopie das Abklingverhalten der Funktion $\mathrm{M}(\mathrm{t})$ verfolgt werden kann. Hierzu wird die Position des Echo-Maximums in Abhängigkeit von $t_{23}$ berechnet. Dieses wird durch Nullsetzen der ersten Ableitung von I $\left(t_{23}, t_{12}\right)$ nach $t_{12}=\tau$ bestimmt $[74]$ und definiert eine Funktion $S\left(t_{23}\right)$ durch:

$$
\left.\frac{\mathrm{dI}\left(\mathrm{t}_{23}, \mathrm{t}_{12}\right)}{\mathrm{dt}_{12}}\right|_{\mathrm{t}_{12}=\mathrm{S}\left(\mathrm{t}_{23}\right)}=0
$$

\section{Gleichung 4.60}

Diese neue Funktion $\mathrm{S}\left(\mathrm{t}_{23}\right)$ wird Echo-Peakshift-Funktion genannt. Die Gleichung 4.58 vereinfacht sich dadurch zu:

$$
\begin{aligned}
& \mathrm{a} \cdot \mathrm{M}_{\text {slow }}^{\prime}\left(\mathrm{t}_{23}\right) \cdot \exp \left[-\Delta^{2} \mathrm{a}^{2} \mathrm{M}^{\prime}{ }_{\text {slow }}\left(\mathrm{t}_{23}\right)^{2} \mathrm{~S}\left(\mathrm{t}_{23}\right)^{2}\right]-\Delta \cdot S\left(\mathrm{t}_{23}\right) \times \\
& {\left[1-\mathrm{a}^{2}\left(\mathrm{M}_{\text {slow }}^{\prime}\left(\mathrm{t}_{23}\right)\right)^{2}\right]\left\{\sqrt{\pi}+2 \cdot \operatorname{erf}\left[\Delta \cdot \mathrm{a} \cdot \mathrm{M}_{\text {slow }}^{\prime}\left(\mathrm{t}_{23}\right) \cdot \mathrm{S}\left(\mathrm{t}_{23}\right)\right]\right\}=0}
\end{aligned} .
$$

Gleichung 4.61

Da der schnelle Anteil in den meisten Fällen dominiert, werden nur die linearen Komponenten von a berücksichtigt:

$$
\mathrm{a} \cdot \mathrm{M}_{\text {slow }}^{\prime}\left(\mathrm{t}_{23}\right)=\frac{\Delta \sqrt{\pi} \cdot \mathrm{S}\left(\mathrm{t}_{23}\right)}{1+2 \Delta^{2} \mathrm{~S}^{2}\left(\mathrm{t}_{23}\right)}
$$

\section{Gleichung 4.62}

Im Grenzfall hoher Temperaturen folgt unter der Annahme, dass $\Delta^{2} S^{2}\left(t_{23}\right)<<1$ gilt:

$$
\mathrm{M}_{\text {slow }}\left(\mathrm{t}_{23}\right)=\mathrm{M}_{\text {slow }}^{\prime}\left(\mathrm{t}_{23}\right)=\mathrm{M}^{\prime \prime}{ }_{\text {slow }}\left(\mathrm{t}_{23}\right)=\frac{\Delta \sqrt{\pi}}{\mathrm{a}} \mathrm{S}\left(\mathrm{t}_{23}\right) \text {. }
$$

\section{Gleichung 4.63}


Bei Gleichung 4.63 handelt es sich um einen direkten Zusammenhang zwischen dem 3-Puls-Echo-Peakshift (3-PEPS) und dem langsamen Anteil der System-BadKorrelationsfunktion $\mathrm{M}(\mathrm{t})$. Bei diesem Ergebnis ist von Bedeutung, dass es keine Abhängigkeit von der Populationsrelaxationszeit $\mathrm{T}_{1}$ gibt.

$\mathrm{Zu}$ frühen Zeiten lässt sich allerdings zeigen, dass die Korrelationsfunktion nicht mehr der 3-PEPS-Kurve entspricht. Eine Taylor-Reihenentwicklung der Korrelationsfunktion $M(t)$ um $t=0$ liefert eine Steigung von Null [74, 77]:

$$
\mathrm{M}^{\prime}(\mathrm{t})=1-\frac{\omega_{0}^{2} \mathrm{t}^{2}}{2}
$$

\section{Gleichung 4.64}

Die Steigung der 3-PEPS-Kurve ergibt dagegen folgenden Ausdruck [74-76]:

$$
\lim _{t_{23} \rightarrow+0}\left(\frac{d S\left(t_{23}\right)}{d_{23}}\right)=\frac{-1}{2+\frac{4}{3} \Delta^{2} S^{2}\left(t_{23}=0\right)\left[1+2 \omega_{0}^{2} S^{2}\left(t_{23}=0\right)\right]} .
$$

\section{Gleichung 4.65}

Die Steigung für $t_{23}=0$ ist folglich negativ. Der genaue Zeitpunkt des Übergangs

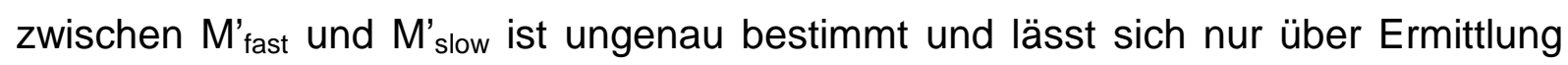
und Überprüfung der Korrelationsfunktion erhalten.

Zusammenfassend lässt sich festhalten, dass mit Ausnahme der Kurzzeitdynamiken der 3-Puls-Echo-Peakshift zur Bestimmung der System-Bad-Korrelationsfunktion verwendet werden kann. Der direkte Zusammenhang und die einfache Detektion des Signals haben diese Spektroskopie zu einem häufig eingesetztem Werkzeug zur Bestimmung der Solvatationsdynamik in molekularen Flüssigkeiten [78-80] gemacht. In den später zu beschreibenden Experimenten zur Solvatationsdynamik an Lipidmembran-Grenzschichten wurde ebenfalls diese Technik verwendet. 


\subsection{Transiente Gitter Spektroskopie}

Bei der Erzeugung und der Abfrage eines transienten Gitters („transient grating“; TG) handelt es sich wie bei der Photon-Echo-Spektroskopie um Vier-WellenMischen (4WM). Analog der Photon-Echo-Spektroskopie wird das Signal in Form des zeitintegrierten Quadrates der Polarisation dritter Ordnung $\mathrm{P}^{(3)}$ gemessen [81]:

$$
\mathrm{I}_{\mathrm{TG}}\left(\mathrm{t}_{23}\right)=\int_{0}^{\infty}\left|\mathrm{P}^{(3)}\left(\mathrm{t}_{12}=0, \mathrm{t}_{23}, \mathrm{t}\right)\right|^{2} \mathrm{dt}
$$

\section{Gleichung 4.66}

mit $\mathrm{P}^{(3)}$ aus Gleichung 4.44. Im Gegensatz zum 2-PE $\left(\mathrm{t}_{23}=0\right)$ und 3-PE, bei dem sowohl $t_{23}$ als auch $t_{12}$ variiert werden, ist beim transienten Gitter Experiment $t_{12}=0$. Die Lichtwellen 1 und 2 werden somit zeitgleich eingestrahlt. In den im Kapitel 4.4, Abbildung 4.4 gezeigten Feynmann-Diagrammen bedeutet dies, dass sich das System nach der zweiten Materie Feldwechselwirkung im Grundzustand bzw. im angeregten Zustand $(|g><g| ;|e><e|)$ befindet. Ist die Bandbreite der Laserpulse größer als der Energieabstand benachbarter Schwingungszustände bzw. die Pulsdauer kleiner als die Schwingungsperiode, so wird eine kohärente Wellenpaketdynamik induziert.

Der dritte Puls erzeugt nochmals eine elektronische Kohärenz (siehe FeynmannDiagramme Abbildung 4.4). Die resultierende Polarisation 3. Ordnung ist wiederum verantwortlich für die Emission von Licht in Richtung $k 4=-k 1+k 2+k 3$. Das zeitlich integrierte Betragsquadrat dieser Polarisation als Funktion von $t_{23}$ ist durch die Schwingungs-Wellenpaketdynamik moduliert. Unter Berücksichtigung der Signalbeiträge, in denen der dritte Puls zeitgleich oder vor den beiden anderen Laserpulsen eingestrahlt wird kann das Signal $\mathrm{I}_{\mathrm{TG}}$ in folgender Form beschrieben werden [82]:

$$
I_{T G}\left(t_{23}\right)=\left|A \cdot \exp \left(-\frac{t_{23}^{2}}{\Delta^{2}}\right)+\sum_{i=1}^{3 n-5 \text { oder } 3 n-6} B_{i} \cos \left(\omega_{i} t_{23}+\phi_{i}\right)+C \cdot R_{r o t}\right| .
$$

\section{Gleichung 4.67}


Der erste Term, eine Gaußfunktion mit der Amplitude A, berücksichtigt die oben erwähnten Anteile des Signals für negative Zeiten $t_{23}$ und den Bereich, in dem die drei Pulse zeitgleich in der Probe eintreffen. $\Delta$ bestimmt die zeitliche Breite dieses Anteils. Die weiteren Terme repräsentieren die Wellenpaketdynamik. Die Summe (3n-5 für lineare beziehungsweise 3n-6 für gewinkelte Moleküle, $n=$ Anzahl der Atome) gibt die Schwingungen des Chromophors im Grund- und angeregten Zustand wieder. Die Schwingungen werden durch die zeitabhängige Amplitude $B_{i}$ und die Phase $\phi_{i}$ der Moden i mit der Frequenz $\omega_{i}$ beschrieben. Zuletzt wird die Rotation des Farbstoffs mit einer Amplitude $C$ und einem Term $R_{\text {rot }}$ berücksichtigt [82].

TG Messungen sind äquivalent zum Pump-Probe-Experiment, bei dem in gleicher Weise sowohl Grundzustand als auch angeregter Zustand abgefragt werden. Beim Pump-Probe-Experiment kommen die beiden ersten Feldwechselwirkungen vom gleichen Puls, dem Pump-Puls. Das Signal wird daher in die Richtung -k1+k1+k3 abgestrahlt, welches der Richtung k3 des 3.Feldes entspricht. Beim Pump-ProbeExperiment ist daher die Polarisation dritter Ordnung identisch zu der des transienten Gitters. Die Detektion der Polarisation 3. Ordnung geschieht dann allerdings in „Vorwärtsrichtung", also in Interferenz mit dem 3. E-Feld (Optische HeterodynDetektion).

Durch ein physikalisches Bild lässt sich das transiente Gitter, wie in Abbildung 4.7 a.) gezeigt, veranschaulichen [82]. In diesem Bild überlappen die eingestrahlten elektromagnetischen Felder in der Probe. Auf Grund von konstrukiver und destruktiver Interferenz bildet sich ein transientes Gitter in der Probe aus. Dieses Gitter zerfällt mit der Zeit durch Populationsrelaxation und wird durch die Schwingungen der Moleküle moduliert. 


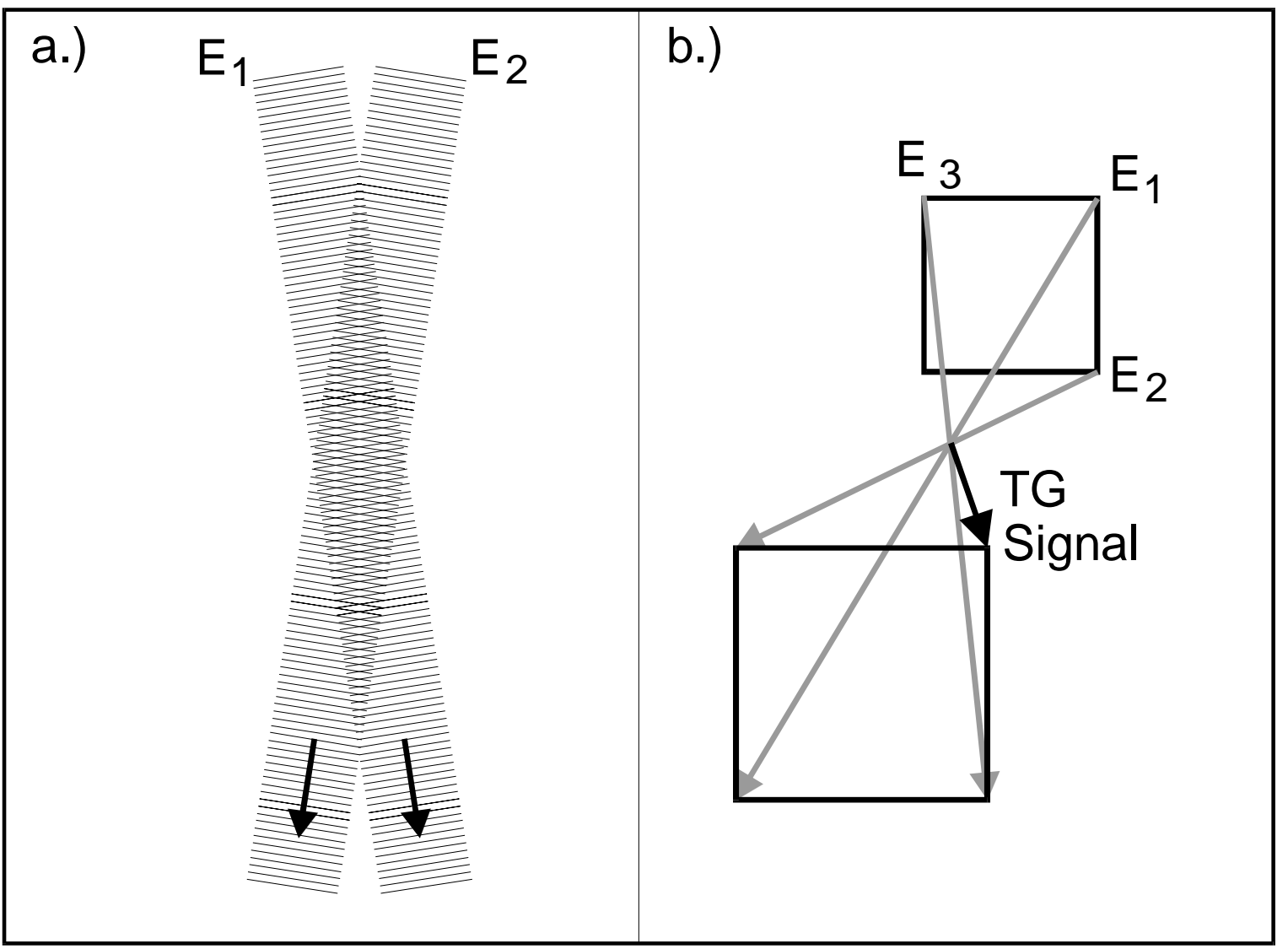

Abbildung 4.7: Transientes Gitter: a.) schematisches Bild; b.) Boxcargeometrie

Der dritte Strahl wird nun im Bragg Winkel [83] an diesem Gitter gebeugt. In Abbildung 4.7 b.) ist die Signalabstrahlung für den Fall der Boxcargeometrie gezeigt. Die zuvor eingestrahlten Felder $E_{1}, E_{2}, E_{3}$ erzeugen ein Signal in Richtung der noch freien Ecke eines Quadrates k4 = -k1+k2+k3. 


\section{EXPERIMENTELLER TEIL}

\subsection{Lasersystem}

Das bei den Messungen eingesetzte Lasersystem besteht aus einem kommerziellen regenerativen Verstärkersystem (Clark Inc., CPA-2001) (Kapitel 5.1.1) und einem nichtkollinearen optisch-parametrischen Verstärker (NOPA) (Kapitel 5.1.2).

\subsubsection{Regenerativer Verstärker}

Der CPA-2001 (CPA = „chirped pulse amplifier“) [84-88] emittiert Laserpulse mit einer Pulsdauer von $150 \mathrm{fs}$ bei einer Zentralwellenlänge von $775 \mathrm{~nm}$. Die Repetitionsrate der Laserpulse beträgt $1 \mathrm{kHz}$ und die Pulsenergie ca. $850 \mu \mathrm{J}$.

Die Pulse werden in einem ringförmigen Resonator mit einer mit Erbiumionen $\left(\mathrm{Er}^{3+}\right)$ dotierten Glasfaser als aktives Medium erzeugt [89-91]. Die Glasfaser wird mit der Strahlung eines Diodenlasers gepumpt, der Licht bei einer Zentralwellenlänge von $980 \mathrm{~nm}$ mit einer Leistung von ca. 900 mW emittiert. Die Einkopplung der Strahlung des Diodenlasers in die Glasfaser erfolgt mit einem WDM-Koppler („wavlengthdivision mupltiplexer“, WDM). Danach emittiert die Faser Licht bei einer Wellenlänge von $1550 \mathrm{~nm}$. Durch den Einsatz von polarisationsadditiver Pulsmodenkopplung [90] („polarisation additive pulse mode-locking“, P-APM) wird der Faserlaser in den Pulsmodus versetzt. Diese Variante der Modenkopplung nutzt die intensitätsabhängige Rotation der Polarisation einer elliptisch polarisierten Lichtwelle in einem Kerr-Medium aus. Die Polarisation der maximalen Intensität der umlaufenden Lichtwelle wird stärker gedreht als die Flanken, so dass durch eine Kombination aus $\lambda / 2$ Platte und Polarisator nur große Intensitäten des Lichtpulses verstärkt werden.

Der emittierte Puls wird nach Auskopplung aus dem Oszillator mit einer Prismenkombination zeitlich auf ca. $100 \mathrm{fs}$ komprimiert und in einem Kristall frequenzverdoppelt $(775 \mathrm{~nm})$. Anschließend wird der Puls erneut gestreckt und in einen regenerativen Verstärker (RGA) eingekoppelt. Dieser beinhaltet einen mit Titanionen $\left(\mathrm{Ti}^{3+}\right)$ dotierten Saphir-Kristall (Ti:Sa) als Lasermedium. Als Pumpquelle dient ein im CPA-2001 eingebauter frequenzverdoppelter Nd:YAG-Laser (Wellenlänge 532 nm, Pulsenergie 8 mJ). Die Pulse werden mit einer Frequenz von 
$1 \mathrm{kHz}$ mit einer Pockelszelle in den RGA ein- und ausgekoppelt und anschließend wieder zeitlich komprimiert. Die vor der regenerativen Verstärkung durchgeführte Pulsstreckung ist notwendig, um unterhalb der Zerstörungsschwelle des Verstärkungsmediums Ti:Sa zu bleiben [84, 92-94].

Die Stabilität des Lasers und die Anzahl der Umläufe im RGA werden durch die Beobachtung der Intensität der sich im RGA aufbauenden Pulse mittels einer schnellen Photodiode (Anstiegszeit < 500 ps) und einem Oszilloskop (Tektronix 485, $350 \mathrm{MHz}$ ) kontrolliert. Am Ausgang des CPA-2001 stehen Pulse mit einer Energie von $850 \mu \mathrm{J}$ und eine Dauer von 150 fs zur Verfügung. Diese werden zum Pumpen des nachfolgend beschriebenen nichtkollinearen optisch-parametrischen Verstärkers verwendet.

\subsubsection{Nichtkollinearer optisch-parametrischer Verstärker (NOPA)}

Mit einem NOPA („noncollinear optical parametrical amplifier“) ist es möglich, ultrakurze Laserpulse im gesamten sichtbaren Spektralbereich zu generieren [95100]. Der NOPA eignet sich daher für Photon-Echo-Experimente, da bei diesen Experimenten das Laserspektrum dem über $100 \mathrm{~nm}$ breiten Anregungsspektrum des Farbstoffs angepasst werden muss.

\subsubsection{Theorie der parametrischen Verstärkung im NOPA}

Bei der parametrischen Verstärkung $[101,102]$ werden zwei Laserpulse, ein Pump$\left(E_{P}\right)$ und ein Seedpuls $\left(E_{S}\right)$, mit unterschiedlicher Intensität in ein nichtlineares Medium eingestrahlt (Abbildung 5.1).

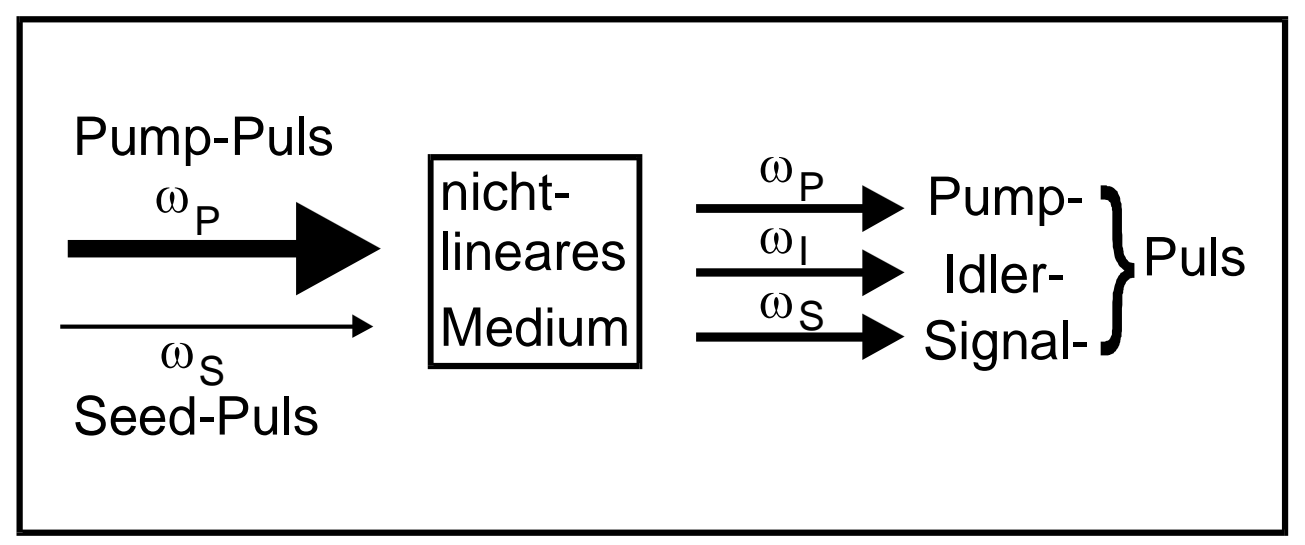

Abbildung 5.1: Parametrische Verstärkung [102] 
Die Energie des intensiveren Pumppulses wird bei der parametrischen Verstärkung auf zwei Lichtfelder mit geringerer Frequenz ( $E_{S}$ Signal und $E_{l}$ Idler) transferiert. Eingeleitet wird der Energietransfer durch einen im Vergleich zum Pumppuls schwachen Seedpuls. Im vorliegenden Aufbau wird ein Weißlichtkontinuum verwendet, das die Funktion des Signalpulses übernimmt (Abbildung 5.1). Bei der parametrischen Verstärkung handelt es sich um eine Drei-Wellen-Mischung, für die Impulserhaltung (Phasenanpassung)

$$
\overrightarrow{\mathrm{k}}_{\mathrm{p}}=\overrightarrow{\mathrm{k}}_{\mathrm{s}}+\overrightarrow{\mathrm{k}}_{\mathrm{i}} \quad \text { mit } \quad \mathrm{k}=\mathrm{n}(\omega) \cdot \frac{\omega}{\mathrm{c}}
$$

\section{Gleichung 5.1}

und Energieerhaltung

$$
\omega_{\mathrm{p}}=\omega_{\mathrm{s}}+\omega_{\mathrm{i}}
$$

\section{Gleichung 5.2}

gelten. Dabei beschreibt k den Wellenvektor des Lichtfeldes, $\omega$ seine Frequenz und c die Lichtgeschwindigkeit. Die Indizes p, s und i kennzeichnen Pump-, Signal- und Idlerwellen. Die beiden Gleichungen lassen sich gleichzeitig nur in anisotropen Medien erfüllen, in denen sich der ordentliche Brechungsindex $n_{0}$ vom außerordentlichen Brechungsindex $n_{e}$ unterscheidet. Zwei Arten der Phasenanpassung werden allgemein genutzt. Bei der Phasenanpassung vom Typ I sind Signal- und Idlerwelle bezüglich der optischen Achse des Kristalls entweder beide parallel (ordentlich, o) oder beide senkrecht (außerordentlich, e) polarisiert, während bei der Typ II-Phasenanpassung entweder der Signalpuls oder der Idlerpuls ordentlich und der jeweils andere außerordentlich polarisiert ist.

Der Phasenanpassungswinkel $\alpha_{p}$ (Abbildung 5.3 a.)) zwischen Pumpstrahl und optischer Achse des anisotropen Kristalls bei der Phasenanpassung nach Typ I ergibt sich zu [103, 104]: 


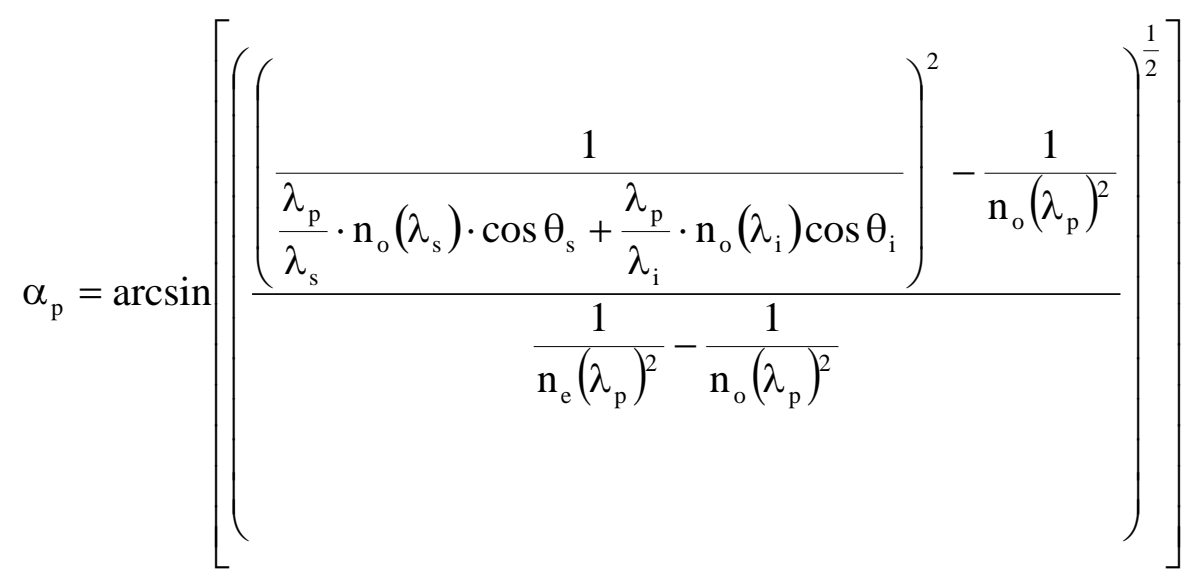

Gleichung 5.3: Phasenanpassungswinkel $\alpha_{P}$ : a.) kollinear

$$
\theta_{\mathrm{i}}=\theta_{\mathrm{s}}=0^{\circ}
$$

b.) nichtkollinear $\quad \theta_{\mathrm{i}} \neq 0^{\circ} ; \theta_{\mathrm{s}} \neq 0^{\circ}$

Bei $\theta_{\mathrm{i}}=\theta_{\mathrm{s}}=0^{\circ}$ spricht man von kollinearer Phasenanpassung. Für eine gegebene Pumpwellenlänge ergibt sich dann je nach gewünschter Signalwellenlänge der Phasenanpassungswinkel. Wird als Pumpquelle das frequenzverdoppelte Licht des CPA-2001 $\left(\lambda_{p}=387.5 \mathrm{~nm}\right.$ ) verwendet, ergibt sich im kollinearen Fall die in Abbildung 5.2 mit $\theta_{s}=0^{\circ}$ gezeichnete Kurve. Die Wellenlängenabhängigkeit des Phasenanpassungswinkels $\alpha_{p}$ im kollinearen Fall ermöglicht nur die Verstärkung eines kleinen Bereiches des sichtbaren Spektrums. Eine Erzeugung ultrakurzer Laserpulse ist daher erschwert. Hinzu kommt der Gruppengeschwindigkeitsunterschied (,group velocity mismatch“; GVM) zwischen Signal- und Idlerwelle $\left(v_{i}>v_{s}\right)$, der zu einem zeitlichen Auseinanderlaufen dieser beiden Wellen führt. Durch die parametrische Verstärkung beider Wellen wird der erzeugte Puls daher zeitlich verlängert. Diese Problematik läßt sich mit einer nichtkollinearen Geometrie (Abbildung 5.3 a.)) lösen. In diesem Fall ergibt sich die Phasenanpassungsbedingung aus Gleichung 5.3 zu:

$$
\mathrm{k}_{\mathrm{p}}=\mathrm{k}_{\mathrm{s}} \cdot \cos \theta_{\mathrm{s}}+\mathrm{k}_{\mathrm{i}} \cdot \cos \theta_{\mathrm{i}}
$$

\section{Gleichung 5.4}




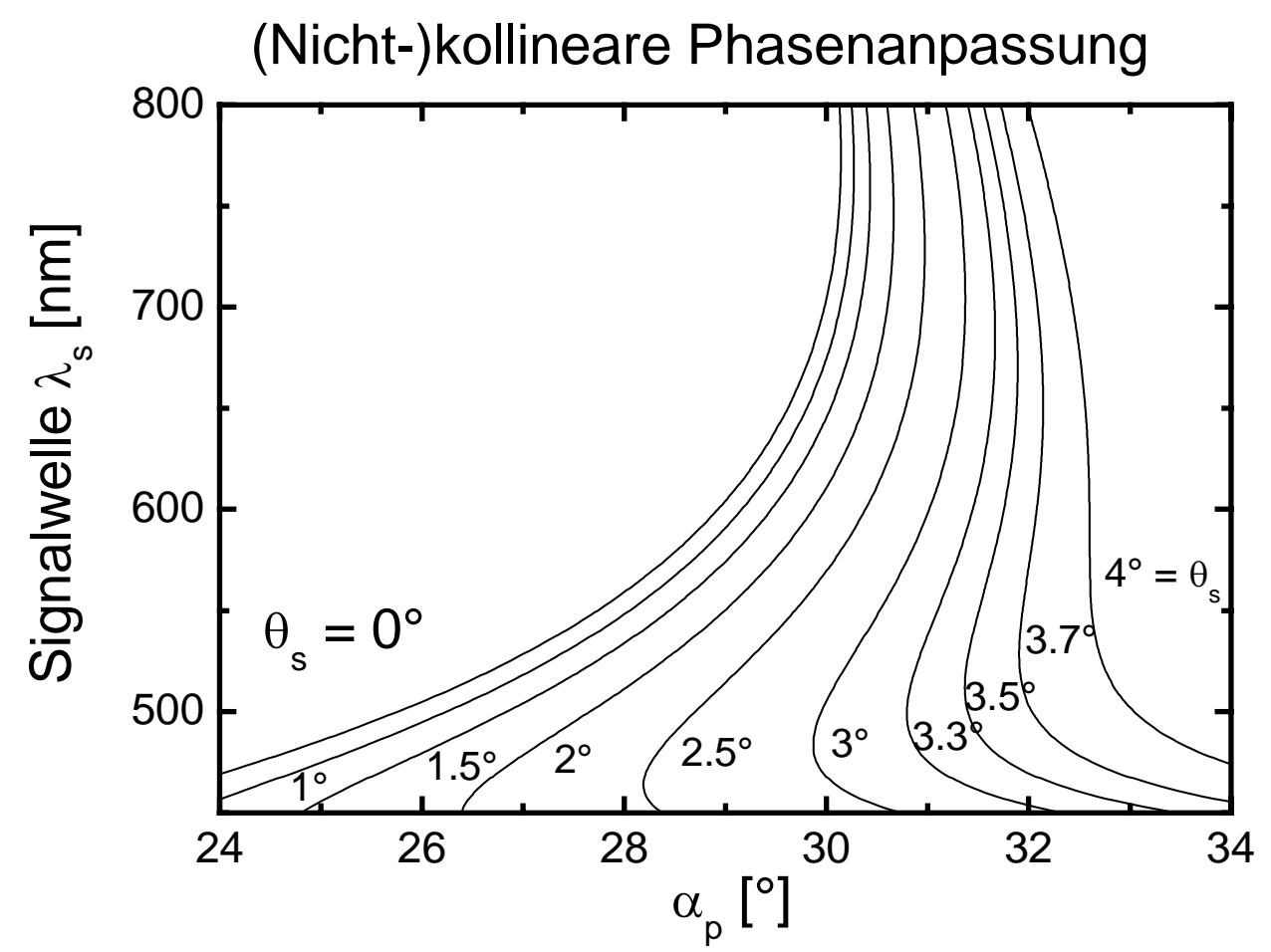

Abbildung 5.2: Phasenanpassungswinkel $\alpha_{p}$ aufgetragen als Funktion der Signalwelle für unterschiedliche Einstrahlungswinkel $\theta_{\mathrm{S}}$ des Seedpulses [105].

Abbildung 5.2 zeigt, dass für einen Winkel von $\theta_{\mathrm{s}}=3.7^{\circ}$ zwischen Pump- und Signalwelle, der Phasenanpassungswinkel $\alpha_{p}$ zwischen Pumpstrahl und optischer Achse des Kristalls im Bereich von $500 \mathrm{~nm}$ bis $750 \mathrm{~nm}$ sich nur um $2^{\circ}$ ändert. In dieser Geometrie ist somit eine spektral breitbandige Verstärkung möglich.

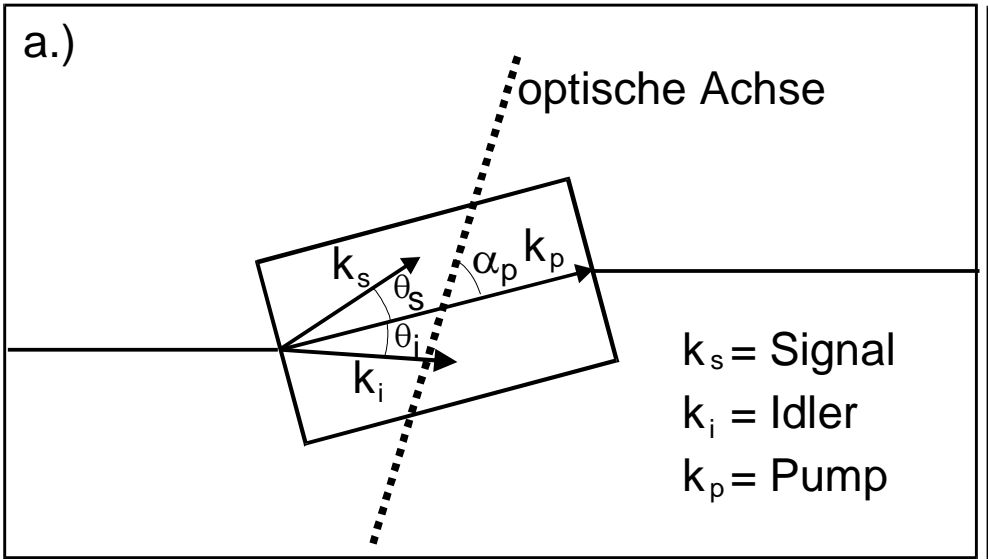

b.)

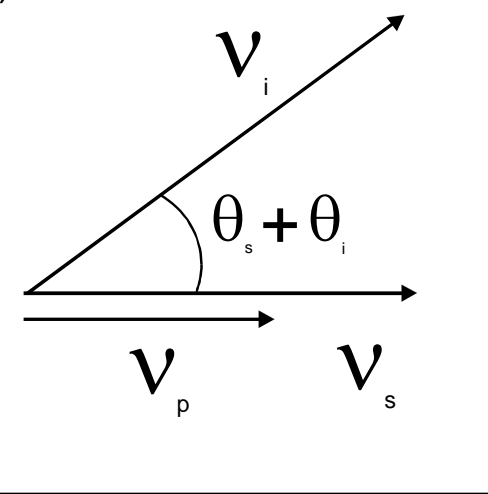

Abbildung 5.3: Nichtkollineare optisch-parametrische Verstärkung [106, 107]

a.) Wellenvektoren b.) Geschwindigkeitsvektoren; $\alpha, \theta=$ Winkel;

$v=$ Gruppengeschwindigkeiten der einzelnen Lichtwellen; $\mathbf{k}=$ Wellenvektor. 
Neben dem über einen großen Teil des sichtbaren Spektralbereich nahezu gleichbleibendem Phasenanpassungswinkel ändert sich bei nichtkollinearer Geometrie auch der Gruppengeschwindigkeitsunterschied (GVM). Für den GVM ist nun nur noch die Projektion (Abbildung 5.3 b.)) der Gruppengeschwindigkeit der Idlerwelle $v_{i}$ auf die Signalwelle von Bedeutung (Gleichung 5.5) [105]:

$$
v_{\mathrm{s}}=v_{\mathrm{i}} \cdot \cos \left(\theta_{\mathrm{s}}+\theta_{\mathrm{i}}\right) \quad \text { mit } \quad \mathrm{GVM}=v_{\mathrm{s}}-v_{\mathrm{i}}
$$

Gleichung 5.5

Es lässt sich zeigen, dass bei einer nichtkollinearen Anordnung mit einem Winkel von $\theta_{\mathrm{s}}=3.7^{\circ}$ der GVM zwischen Signal und Idler bei Signalwellenlängen zwischen $500 \mathrm{~nm}$ und $750 \mathrm{~nm}$ zu keiner weiteren Pulsverbreiterung führt [108].

\subsubsection{Aufbau des NOPA}

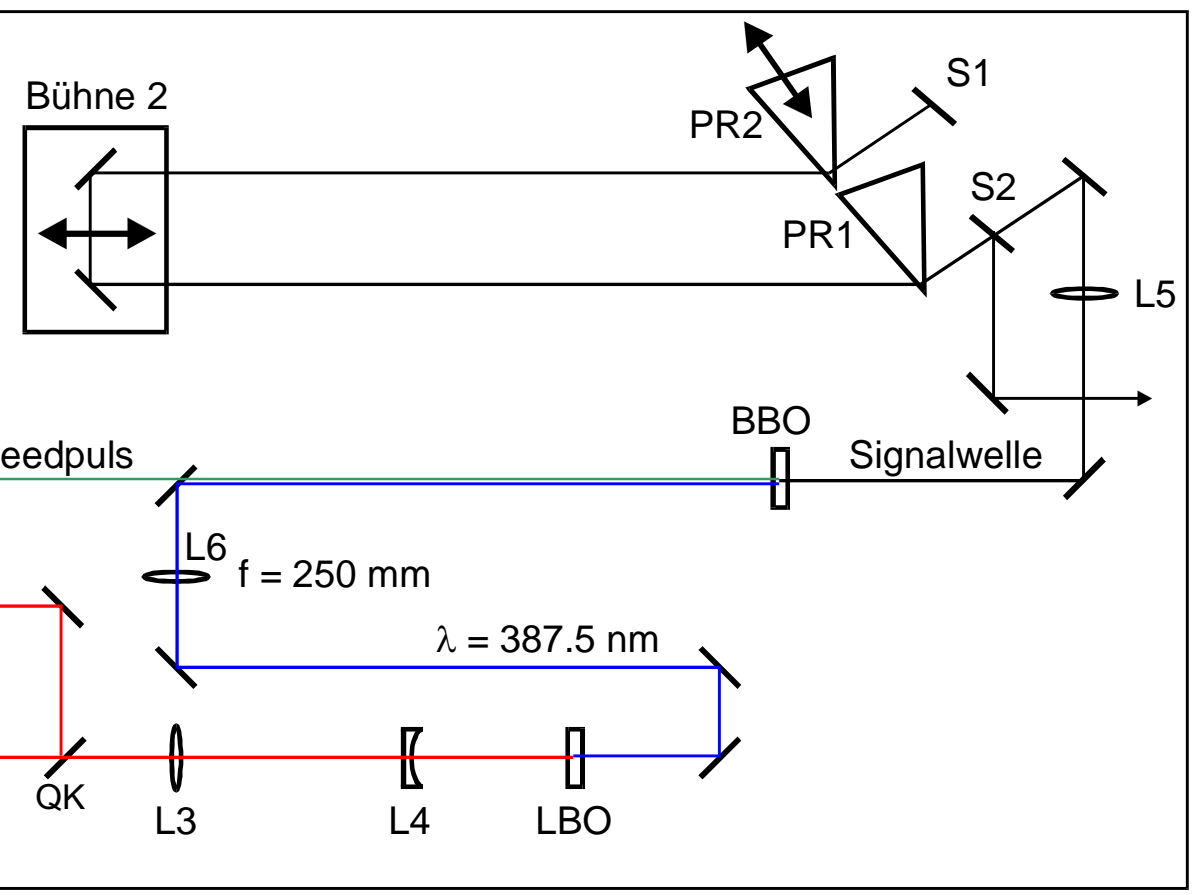

Abbildung 5.4: Aufbau des nichtkollinearen optisch-parametrischen Verstärkers (NOPA);

S1 - S2 = Silberspiegel; L1 - L5 = Linsen; LBO = Lithiumtriborat-Kristall;

BBO = $\beta$-Barium-Borat-Kristall; $Q P=$ Quarzplatte; $Q K=$ Quarzkeil;

PR1 - PR2 = Quarzprismen 
Ein in Abbildung 5.4 schematisch dargestellter nicht-kollinearer optisch-parametrischer Verstärker, dessen Aufbau sich an den Arbeiten von Riedle und Mitarbeitern orientiert [108-110] wird mit $200 \mu \mathrm{J}$ des vom CPA-2001 erzeugten Lichts betrieben. Der Strahldurchmesser des größten Teils des zum Pumpen des NOPAs verwendeten Laserlichtes wird mit zwei Quarzlinsen ( $L 3, f=150 \mathrm{~mm}, \mathrm{~L} 4, \mathrm{f}=-50 \mathrm{~mm}$ ) von ca. $2.4 \mathrm{~cm}$ auf ca. $0.8 \mathrm{~cm}$ mit einem Teleskop verkleinert. Anschließend wird das Laserlicht in einem Lithiumtriborat-Kristall ( $L B O, \theta=29.8^{\circ}$ ) frequenzverdoppelt. Die Energie der Pulse beträgt ca. $85 \mu \mathrm{J}$ und ihre Zentralwellenlänge ist $387.5 \mathrm{~nm}$. Diese Laserpulse gelangen durch eine Linse $(f=250 \mathrm{~mm})$ in einen $\beta$-Barium-Borat-Kristall $\left(\mathrm{BBO}, \theta=32^{\circ}\right)$. Der Fokus des Laserstrahls liegt ungefähr $10 \mathrm{~cm}$ vor diesem Kristall, um die Intensität unterhalb dessen Zerstörungsschwelle zu halten. In einem Winkel von etwa $6.3^{\circ} \mathrm{zu}$ dem Laserstrahl wird zusätzlich der Strahl eines Weißlichtkontinuums als Seedwelle in den BBO-Kristall fokussiert. Der Winkel innerhalb des Kristalls zwischen Pump- und Seedstrahl beträgt $3.7^{\circ}$.

Das Licht für die Erzeugung des Weißlichtkontinuums wird mit einem Quarzkeil (QK) durch Oberflächenreflektion vor dem Teleskop vom Hauptstrahl abgetrennt und mit einer Quarzlinse $(L 1, f=50 \mathrm{~mm})$ in ein $2 \mathrm{~mm}$ dickes Saphirsubstrat fokussiert. Ein gleichmäßiges und stabiles Weißlichtkontinuum („single filament“) [111] läßt sich durch Variation des Abstandes zwischen Linse und Substrat einstellen. Das erzeugte Licht wird über eine weitere Quarzlinse (L2, f=40 mm) und die Verzögerungsstrecke 1 (Bühne 1 in Abbildung 5.4) in den BBO-Kristall fokussiert. Das Weißlichtkontinuum besitzt eine lineare Gruppengeschwindigkeitsdispersion (linearer Chirp). Jeweils der Teil des Weißlichts, der zeitlich mit dem Pumpstrahl überlappt, wird verstärkt, da die Phasenanpassungsbedingung für einen breiten Spektralbereich erfüllt ist (siehe Abschnitt 5.1.2.1). Weil außerdem die Gruppengeschwindigkeit $v_{p}$ des Pumpstrahls kleiner ist als die der Seed- bzw. Signalwelle, überlappen je nach zurückgelegtem Weg innerhalb des Kristalls verschiedene Wellenlängen des Weißlichtkontinuums mit dem Pumppuls. Somit wird ein breites Spektrum verstärkt. Abhängig vom Chirp des Seedstrahls wird in nichtkollinearer Anordnung in einem längerem Kristall daher ein breiteres Spektrum verstärkt als in einem kurzen. Im Anschluss an die parametrische Verstärkung beträgt die Pulsenergie ungefähr $8 \mu \mathrm{J}$. Die Linse L5 $(f=400 \mathrm{~mm})$ hinter dem BBO-Kristall kollimiert den erzeugten Laserstrahl.

Die Zentralwellenlänge des NOPA Spektrums wird mit der Bühne 1 eingestellt, indem 
die gewünschte Frequenz des Seedstrahls mit dem Pumpstrahl in der Mitte des BBO-Kristalls zeitlich überlappt. Da der Chirp der erzeugten Pulse nahezu linear ist, können diese mit einem Prismenpaar zeitlich komprimiert werden [92]. Der Laserstrahl wird hierzu durch ein Quarzprisma (PR1) über eine variable Dispersionsstrecke (Bühne 2) geleitet, die den Strahl auf ein zweites Quarzprisma (PR2) lenkt. Die nachfolgende, aus zwei Spiegeln bestehende Anordnung S1, reflektiert den Strahl einen Zentimeter tiefer wieder auf dem umgekehrten Weg auf den Spiegel S2 zurück. Von hier wird der nun zeitlich komprimierte Laserpuls in das Experiment geleitet.

In Abbildung 5.5 a.) sind Spektren des eingesetzten NOPAs mit unterschiedlichen Zentralwellenlängen $z u$ sehen. Diese wurden mit einem Polychromator (Monochromator: Bausch \& Lomb $f=100$ mm, Gitter $1350 \mathrm{l} / \mathrm{mm}$; Detektor S3901, Hamamatsu) aufgenommen. In den Experimenten wurde die Breite des Laserspektrums an das Absorptionsspektrum des jeweils verwendeten Farbstoffs mit einer Quarzplatte (QP) angepasst. Diese bestimmt je nach Dicke die Größe des Chirps des zum Seeden verwendeten Lichtes und damit die Breite des verstärkten Spektrums im BBO Kristall. Die ohne diese Quarzplatte gemessenen Autokorrelationen zu den in Abbildung 5.5 a.) gezeigten Spektren besitzen eine mittlere Breite von 20 fs und sind in Abbildung 5.5 b.) dargestellt.
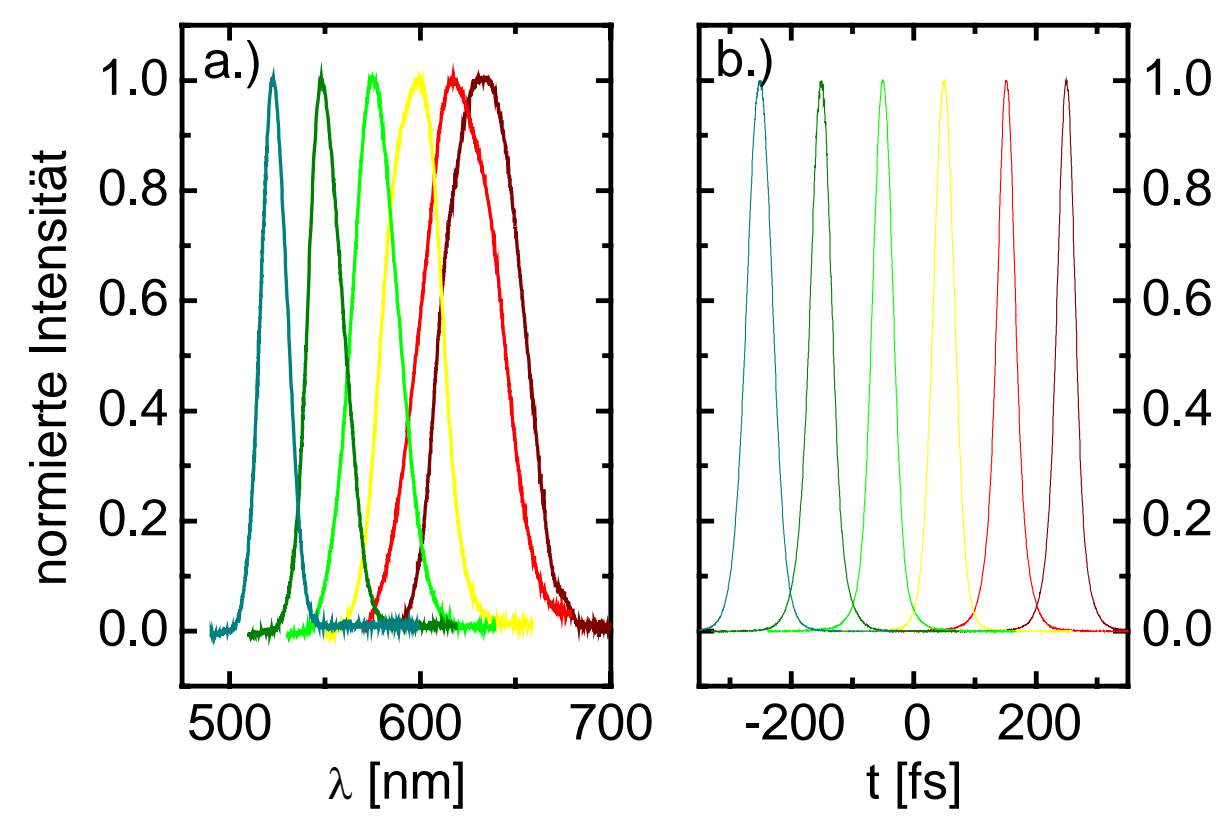

Abbildung 5.5: a.) Spektren des NOPAs. b.) Zugehörige Autokorrelationen.

Die Autokorrelationen sind zur besseren Unterscheidbarkeit auf der Zeitachse gegeneinander verschoben. 


\subsection{Messaufbau}

Für die Photon-Echo-Messungen wurden zwei unterschiedliche Versuchsaufbauten verwendet. Die zunächst beschriebene Dreiecksgeometrie liefert absolute Werte für den Peakshift und zeigt damit auch statische Inhomogenitäten an, während sich die sogenannte Boxcargeometrie durch eine höhere relative Messgenauigkeit auszeichnet. Mit dieser Methode kann der Peakshift allerdings nur relativ bestimmt werden.

\subsubsection{Aufbau der Dreiecksgeometrie}

Den prinzipiellen Aufbau in der Dreiecksgeometrie zeigt Abbildung 5.6 [76]. Der vom NOPA kommende Strahl wird mittels dreier Strahlteilerwürfel (BS) (Linos Photonics, Reflektion $50 \%$, Al Beschichtung, Kantenlänge $5 \mathrm{~mm}$ ) in drei gleich intensive Strahlen $1-3$ aufgespaltet. Die Strahlen 1 und 2 (markiert durch Zahlen in Abbildung 5.6) werden über jeweils eine variable Verzögerungsstrecke (Translationsbühnen 1 und 2, Nanomover, Nanomotion II, Melles Griot) geleitet, während Strahl 3 eine feste Verzögerungsstrecke durchläuft. Die nachfolgende Linse L1 fokussiert die drei Strahlen in die Messzelle (QS-165-1 mm, Hellma). Die räumliche Anordnung der drei Strahlen mit den Wellenvektoren k1 bis k3 auf der Linse ist im Oval der Abbildung 5.6 eingezeichnet. Der zeitliche Abstand zwischen den Pulsen der Strahlen 2 und 3 wird mit $t_{23}$ bezeichnet, der zwischen den Pulsen der Strahlen 1 und 2 mit $t_{12}$. Beide Translationsbühnen erlauben minimale Schrittweiten von $10 \mathrm{~nm}$ und einen maximalen Verstellweg von $25 \mathrm{~mm}$. Ein Computerprogramm, welches die Programmierplattform HPVEE [112] nutzt, steuert die Nanomover. In späteren Messungen konnte der zur Verfügung stehende Messbereich auf ca. 300 ps erweitert werden, in dem die Laserstrahlen 1 und 2 je zweimal über Spiegel auf den zugehörigen variablen Translationsbühnen geleitet wurden. Der Übersichtlichkeit wegen sind diese Details aber nicht in Abbildung 5.6 und der im Rahmen der Boxcargeometrie diskutierten Abbildung 5.7 eingezeichnet. 
Die nichtkollineare Einstrahlung der Laserpulse erzeugt elektromagnetische Felder (z.B. Transient Grating, Photon-Echo) in den durch die Wellenvektoren:

$$
\begin{array}{ll}
\mathrm{k} 4=\mathrm{k} 3+\mathrm{k} 2-\mathrm{k} 1 & \mathrm{k} 4^{\prime}=\mathrm{k} 3-\mathrm{k} 2+\mathrm{k} 1 \\
\mathrm{k} 5=2 \cdot \mathrm{k} 1-\mathrm{k} 2 & \mathrm{k} 5^{\prime}=2 \cdot \mathrm{k} 2-\mathrm{k} 1
\end{array}
$$

Gleichung 5.6

bestimmten Raumrichtungen. Je nach zeitlicher Reihenfolge der eingestrahlten Pulse mit Wellenvektoren k1 bis k3 wird in den Richtungen k4, k4', k5, k5' ein Photon-Echo erzeugt. In Richtung k4 und k4' wird das Drei-Puls-Echo für verschiedene $\mathrm{t}_{23}$ abgestrahlt, in Richtung k5 und k5' ein Zwei-Puls-Echo, das einem Drei-Puls-Echo für $t_{23}=0$ entspricht. Da die erzeugten Felder auf Grund der Phasenanpassungsbedingungen räumlich getrennt sind, können sie einzeln detektiert werden (Spiegel S1 - S4, Detektoren PM1 - PM4 in Abbildung 5.6).

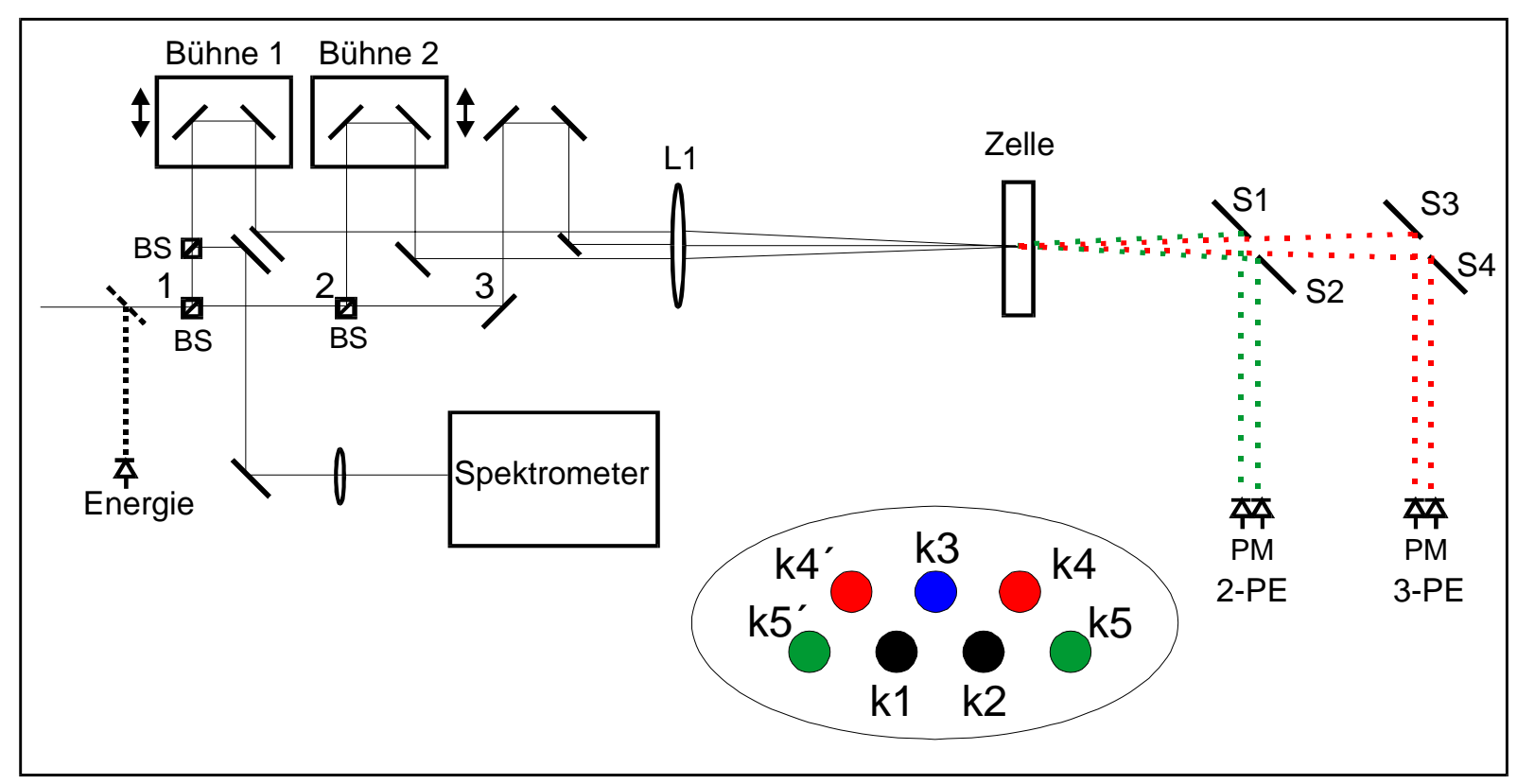

Abbildung 5.6: Experimenteller Aufbau des Photon-Echo-Experiments in Dreiecksgeometrie ; 2PE, 3PE = Photomultiplier zur Messung der Echosignale; BS = Strahlteilerwürfel; L1 = Linsen mit $\mathbf{f}=\mathbf{3 0 0} \mathrm{mm}$; S1 - S4 = Spiegel; k1 - k3 = Laserstrahlenanordnung auf der Linse; k4 - k5 = erzeugte Laserstrahlen. 
In den durchgeführten Experimenten wurde die Pulsenergie des NOPAs durch nachfolgende Neutralglasfilter oder geschlossene Lochblenden auf 10 bis $20 \mathrm{~nJ}$ abgeschwächt. Die dem Experiment zugeleitete Pulsenergie wird zwischen den Messungen mit einer, anhand eines Leistungsmessgeräts geeichten Photodiode überwacht. Um eine gleichbleibende Anpassung des NOPA Spektrums an das Absorptionsspektrum des Farbstoffes zu gewährleisten, wurde ersteres mit dem Polychromator (siehe Abschnitt 5.1.2.2) ständig kontrolliert.

\subsubsection{Aufbau der Boxcargeometrie}

In dem Versuchsaufbau in der Boxcargeometrie [113] wird der vom NOPA kommende Laserstrahl mittels sechs $50 \%$ Strahlteilerwürfel (BS) in sieben gleich intensive Laserstrahlen aufgeteilt. Drei dieser Strahlen (Abbildung 5.7, Strahlen 1-3) werden wie in der Dreiecksgeometrie über zwei variable und eine festen Verzögerungsstrecke geleitet und dann mit der Linse $L 1$ ( $f=300 \mathrm{~mm}$ ) in die Zelle fokussiert. Dieser Teil des Aufbaus wird als Probenteil bezeichnet. Das Oval in Abbildung 5.7 zeigt die Anordnung der Laserstrahlen auf der Linse L1. Das Photon-Echo wird in Richtung k4 beobachtet. Die Phasenanpassung für k4 ist gleich der in der Dreiecksgeometrie (Gleichung 5.6). k1 bis k4 zeigen in die Ecken eines Quadrates.

Gleichzeitig mit der Messung im Probenteil erfolgt im Referenzteil die Aufnahme einer Autokorrelation (Abbildung 5.6). Die Linse $L 2(f=300 \mathrm{~mm})$ fokussiert die Strahlen 4 und 5 in einen BBO-Kristall. Sie werden über die gleichen Translationsbühnen wie die Strahlen 1 und 2 geleitet, so dass der einmal eingestellte Zeitunterschied zwischen dem 3-Puls-Echo-Signal und der Autokorrelation im Referenzteil konstant bleibt. Von den beiden verbleibenden Strahlen wird einer für die Energiekontrolle mittels einer Photodiode verwendet, der andere Strahl für die Überwachung des Spektrums mit dem Polychromator.

Bei Messungen in der Boxcargeometrie lässt sich der Peakshift zur Autokorrelation (AK) nur relativ bestimmen, da sich in der Praxis der Laufzeitunterschied zwischen beiden Messarmen nicht vollständig beseitigen lässt. Bei der Einstellung des Nullpunktes wird ein BBO-Kristall anstelle der Probe im Probenteil eingebaut, so dass eine Autokorrelation gemessen werden kann. Der zeitliche Unterschied zwischen den beiden Autokorrelation wird minimiert. Dies ist mit der Genauigkeit von einer Femtosekunde möglich. Durch Einsetzen der Probe verändert sich jedoch der Nullpunkt geringfügig. 


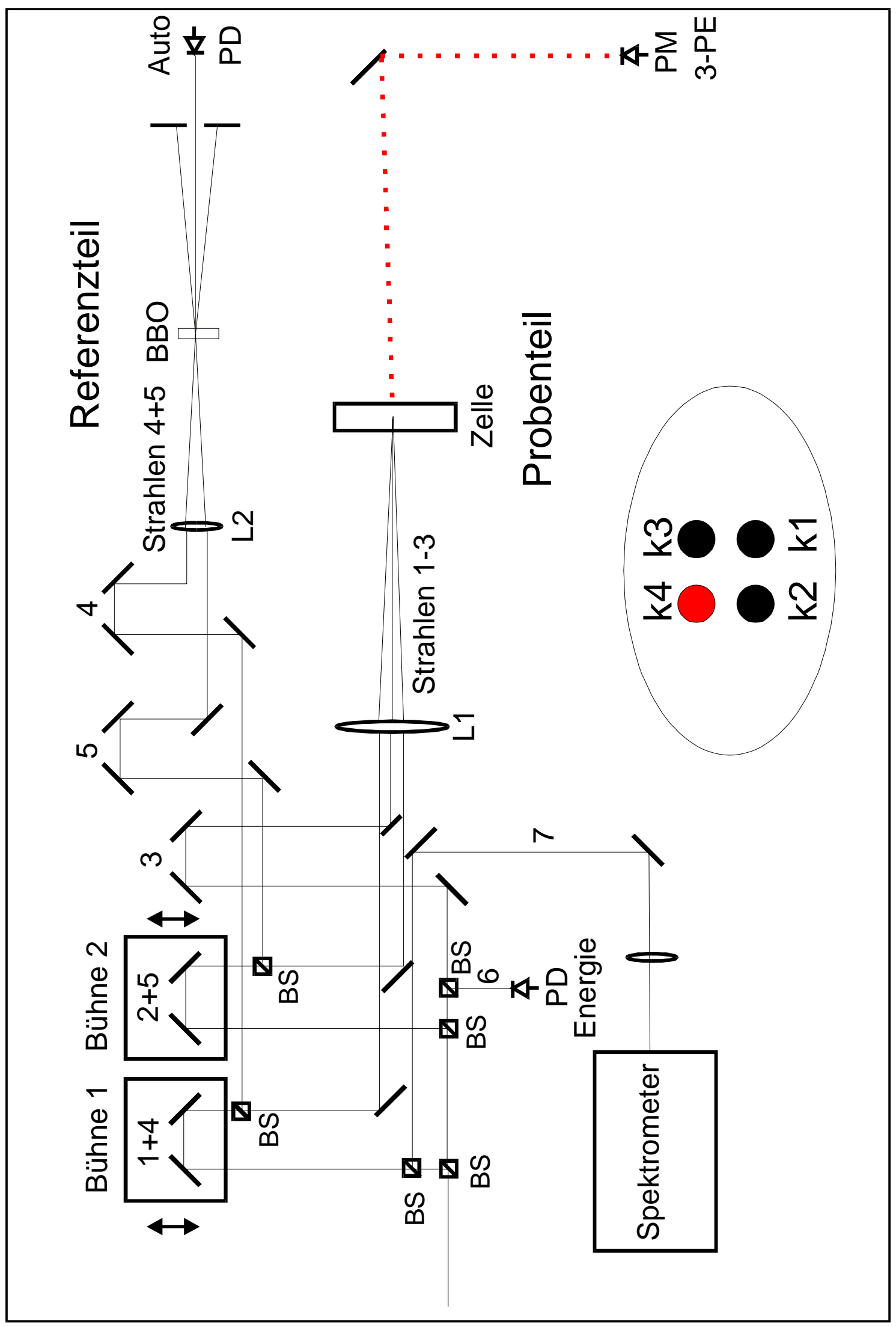

Abbildung 5.7: Boxcargeometrie; PM = Photomultiplier zur Messung des 3-Puls-Echos;

PD = Photodiode zur Messung der Autokorrelation (Auto) und der Energie;

BS = Strahlteilerwürfel; L1, L2 = Linsen mit $f=300 \mathrm{~mm}$;

k1 - k3 = Laserstrahlenanordnung auf der Linse 


\subsubsection{Vergleich zwischen Dreiecks- und Boxcargeometrie}

Die Dreiecksgeometrie misst im Gegensatz zur Boxcargeometrie den absoluten Peakshift. Da hierbei nicht der Peakshift nur bis auf eine Konstante (wie in der Boxcargeometrie) bestimmt ist, gelingt es auch Zeitkomponenten zu bestimmen, deren Abklingzeit die experimentell maximale Verzögerungszeit erreichen. Von Nachteil ist, dass beide Echo-Signale mit Wellenvektoren k4 und k4' für große $t_{23}$ in ihrer Intensität abnehmen. Dies erfordert eine besonders sorgfältige Auswertung, siehe Kapitel 5.3. Ebenso bereitet die genaue Einstellung dieser Geometrie Probleme, so dass der Nullpunkt, der durch die beiden Zwei-Puls-Echos in den Richtungen der Wellenvektoren k5 und k5' ermittelt wird, selten dem Nullpunkt der Drei-Puls-Echos entspricht.

Der Vorteil der Boxcargeometrie ist die effektivere Rauschunterdrückung bei der Peakshiftbestimmung, da ein Bezugspunkt aus der mit gutem Signal-Rauschverhältnis unabhängig von $\mathrm{t}_{23}$ aufgenommenen Autokorrelation bestimmt wird (siehe Kapitel 5.3). Außerdem bereitet die Einstellung der Geometrie nicht die geschilderten Probleme der Dreiecksgeometrie.

Weitere Unterschiede in den beiden beschriebenen Aufbauten ergeben sich aus der Anzahl der Laserstrahlen und benötigten Detektoren. Die Boxcargeometrie benötigt fünf Strahlen und zwei Detektoren, die Dreiecksgeometrie drei Strahlen und vier Detektoren. Insbesondere in Experimenten, wo die zur Verfügung stehende Laserleistung geringer (z.B. im infraroten oder blauen Spektralbereich), die im Experiment benötigte aber größer ist als im vorliegenden Fall, kann sich der Einsatz des Aufbaus in Dreiecksgeometrie als vorteilhaft erweisen. 


\subsection{Experimentsteuerung und Detektion der Signale}

Die Durchführung der Messungen erfordert die Aufnahme der Photon-Echo Signale in Abhängigkeit der Verzögerungszeiten $t_{12}$ und $t_{23}$. Die Ansteuerung der Translationsbühnen der Verzögerungsstrecken erfolgt mit einem in HPVEE [112] geschriebenen Computerprogramm. Dieses Programm fährt die Bühnen 1 und $2 \mathrm{zu}$ einer festen Verzögerungszeit $t_{23}$ und führt dort eine Photon-Echo-Messung für verschiedene Zeiten $t_{12}$ durch. Die Signale werden mit Photodioden (S1336-8BQ, Hamamatsu) beziehungsweise Photomultipliern (R928, Hamamatsu, 7 Dynoden beschaltet, Hochspannung $700 \mathrm{~V}$ ) mit nachfolgenden Integratorschaltungen beobachtet und mit einer A/D-Wandlerkarte (DT 3016; 16 Bit, Data Translation) digitalisiert. Um das Signal-Rauschverhältnis zu verbessern, wurden zu jeder Verzögerungszeit $t_{23}$ zehn bis fünfzehn Messkurven addiert. So erhaltene typische Photon-Echo-Intensitäten als Funktion von $\mathrm{t}_{12}$ sind in Abbildung 5.8 dargestellt.

Die Angabe der Peakshifts erfordert eine genaue Bestimmung der Zeit $t_{12}$, bei der die Photon-Echo Intensität maximal wird. Dazu werden die in Abbildung 5.8 repräsentativ dargestellten Messkurven geglättet, in dem jedem Datenpunkt der Mittelwert der Intensität aus den 15 am nächsten liegenden Punkten auf jeder Seite zugeordnet wird. Anschließend wird der Bereich des Signals, der eine normierte Intensität größer als 0.7 aufweist, an ein Polynom dritten Grades angepasst und die Zeit $\mathrm{t}_{12}$ des Maximums des Polynoms bestimmt. Diese Bestimmung des Maximums (Peak) und der anschließend ermittelten Verschiebung der Maxima als Funktion der Zeit $t_{23}$ (Peakshift) wird mit einem in Matlab [114] geschriebenem Programm durchgeführt.

In der Boxcargeometrie bestimmt sich der Peakshift eindeutig, aber nicht absolut, aus der Differenz der Zeiten maximaler Photon-Echo-Intensität (Wellenvektor k4) und Autokorrelationssignal. Wie bereits erwähnt, ist der Peakshift in dieser Anordnung nur bis auf einen konstanten Wert in der Größenordnung von $1 \mathrm{fs}$ festgelegt. Daher wurden alle Werte, die mit dieser Anordnung gemessen wurden, um einen konstanten Betrag korrigiert. Im allgemeinen wurde der Peakshift für $t_{23}=300$ ps gleich Null gesetzt. 

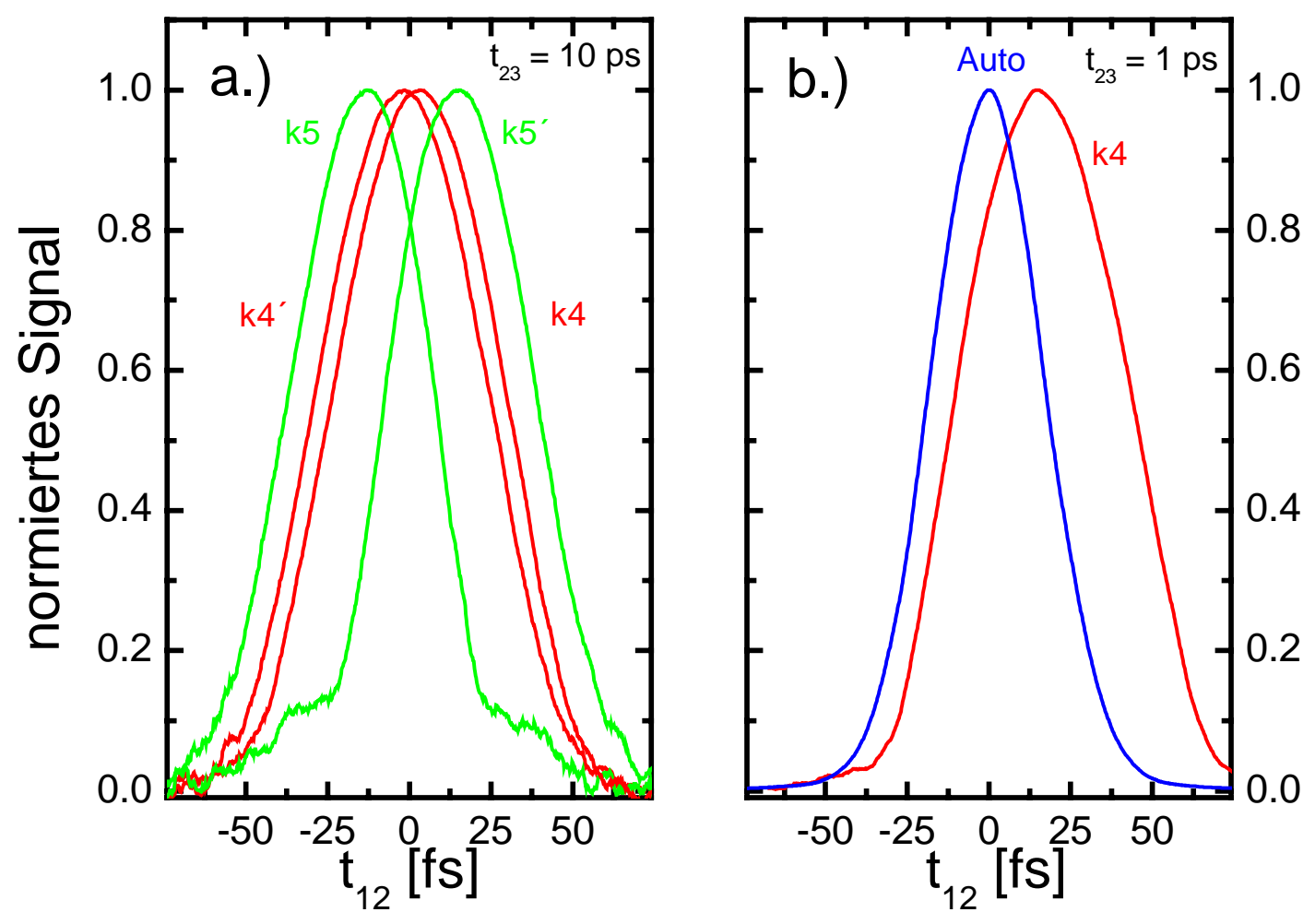

Abbildung 5.8: Photon-Echo-Signale (DiDC ${ }_{18}$ in DMPC) in Abhängigkeit von $t_{12}$ :

a.) Dreiecksgeometrie und b.) Boxcargeometrie; Auto = Autokorrelation;

k4, k4', k5, k5 = Echosignale in den jeweiligen Richtungen.

Im Fall der Dreiecksgeometrie kann der Peakshift allerdings auf zwei Arten bestimmt werden. Zum einen ist er gleich der Hälfte des Abstandes zwischen den beiden Maxima der 3-Puls-Echo-Signale k4 und k4'. Zum anderen lässt er sich aus dem Abstand des 3-Puls-Echo-Maximums zum Nullpunkt ermitteln. Dieser Nullpunkt liegt exakt auf der Hälfte des zeitlichen Abstands der beiden Maxima der Signale in Richtung k5 und k5'. 


\subsection{Temperaturstabilität im Experiment}

\subsubsection{Rotationszelle}

Die zu untersuchende Probe wird in eine thermostatierbare Küvette (165-QS-1mm, Hellma) eingefüllt. Damit es zu keiner lokalen Erhitzung im Fokus der Laserstrahlen oder einem Ausbleichen der Probe kommt, wird die Zelle bewegt. Eine motorbetriebene speziell konstruierte Halterung (siehe Abbildung 5.9) sorgt für eine exzentrische Bewegung der Zelle um den Fokus der Laserstrahlen mit ungefähr 20 $\mathrm{Hz}$, und einem Hub von $2 \mathrm{~mm}$, so dass sich der Fokus in der Zelle mit einer Geschwindigkeit von $250 \mathrm{~mm} / \mathrm{s}$ bewegt. Bei der verwendeten Repetitionsrate von 1 $\mathrm{kHz}$ darf der Fokusdurchmesser daher bis zu $250 \mu \mathrm{m}$ betragen. Dies ist bei den Experimenten gegeben, so dass jeder Laserpuls einen neuen Volumen in der Probe trifft.

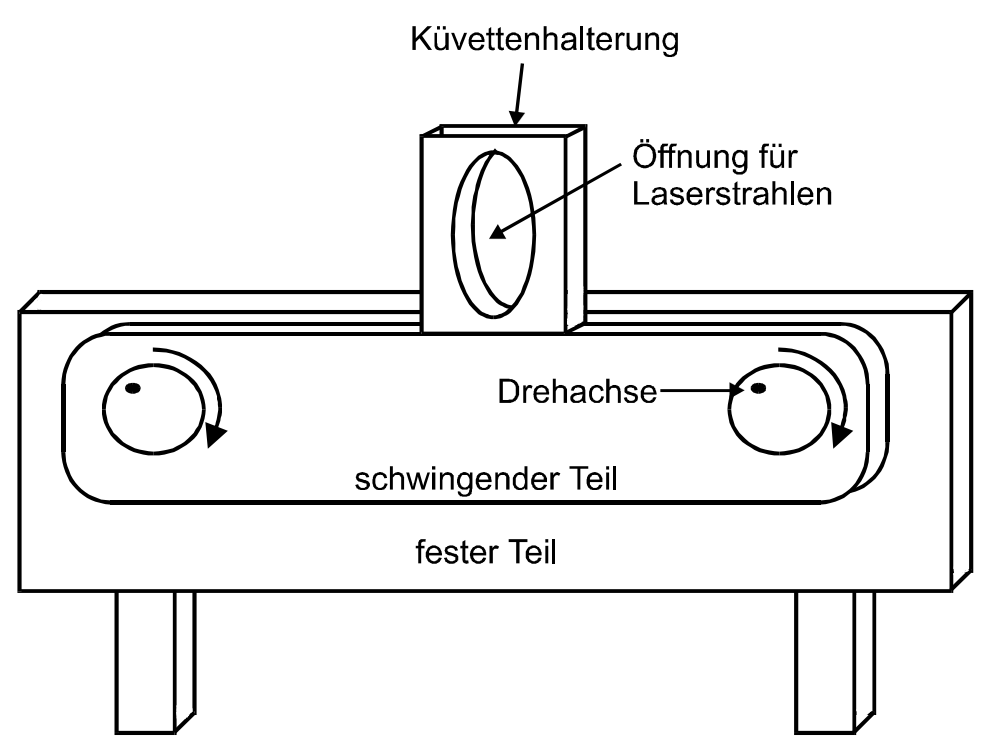

Abbildung 5.9: Zellhalterung mit Rotationsvorrichtung

Die Temperatur wird mit einem Thermostaten (RCS6, Lauda) eingestellt und stabilisiert. Eine genauere Temperaturmessung in der Zelle erfolgt mit einem Thermoelement (Pt100) mit einer Genauigkeit von $0.1^{\circ} \mathrm{C}$. 


\subsubsection{Untersuchung der Erwärmung der Probe im Experiment}

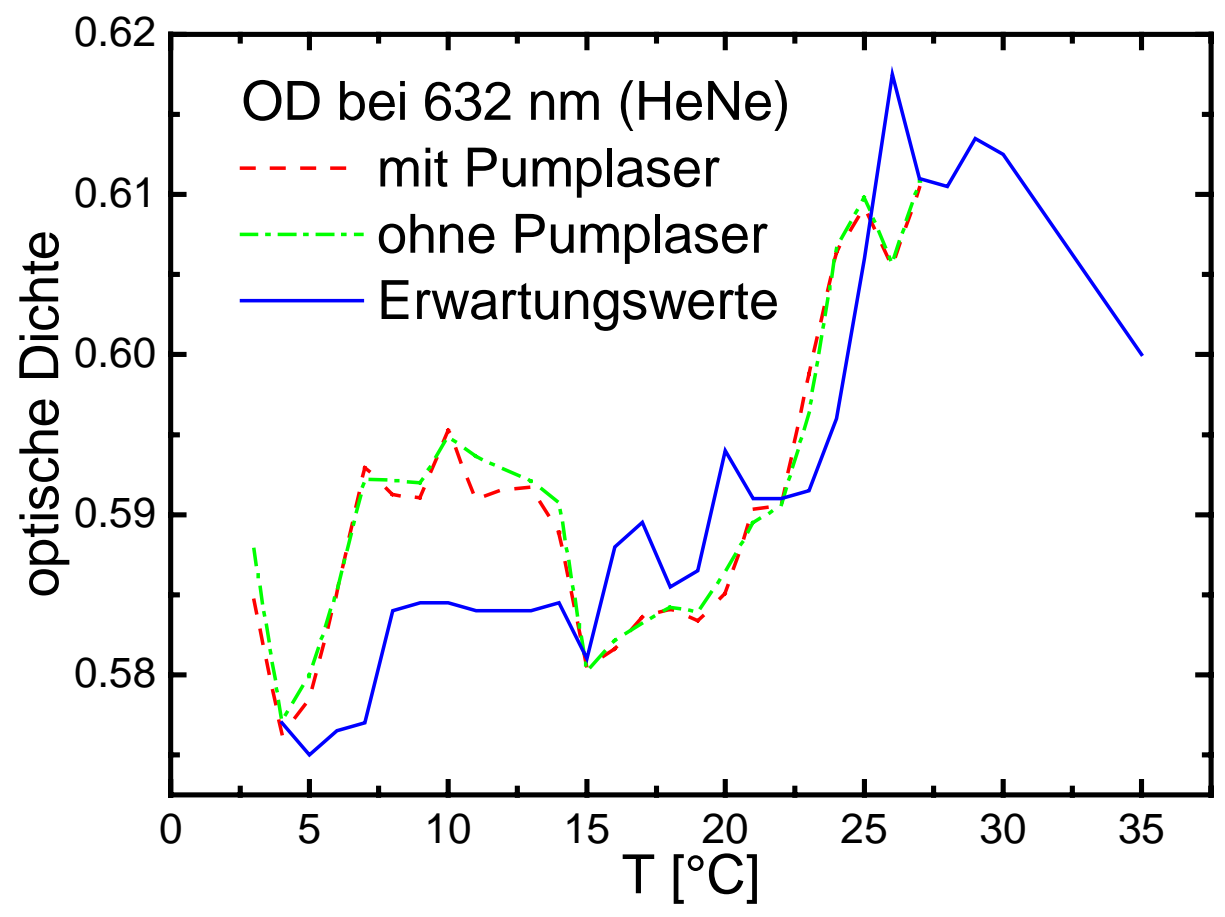

Abbildung 5.10: Temperaturmessung im Fokus

In der vorliegenden Arbeit wird der Einfluss der Membrangrenzschicht auf die Dynamik des Wassers untersucht. Dazu wurden auch Messungen in einem Temperaturbereich durchgeführt, der einen Phasenübergang innerhalb der Membran beinhaltet. Die Kenntnis der genauen Temperatur im Fokus, und ihrer Beeinflussung durch die eingestrahlten Laserstrahlen, ist daher unabdingbar.

Die Messung von Temperaturänderungen durch die drei eingestrahlten Laserpulse gelingt durch die Beobachtung der optischen Dichte der zu untersuchenden Probe. Hierzu wird in der Boxcaranordnung (Abbildung 5.7) der Strahl eines Helium-Neon-Lasers parallel so zu den drei anderen Strahlen justiert, dass alle Strahlen auf der Linse L1 ein Quadrat bilden. Dadurch überlappen alle vier Foki im selben Volumen der Probe. Die Intensität I des HeNe-Laserstrahls wird hinter der Zelle mit einer Photodiode nachgewiesen. Eine weitere Photodiode detektiert die mittels eines Strahlteilers ausgekoppelte relative Intensität $I_{0}$ des HeNe-Lichtes vor der Zelle. Aus den beiden Messgrößen I und $I_{0}$ bestimmt sich eine relative optische Dichte in Abhängigkeit der Temperatur. Das Ergebnis einer solchen Messung zeigt Abbildung 5.10.

Aus dieser Abbildung ist ersichtlich, dass das Photon-Echo-Experiment die Temperatur im Fokus nicht beeinflusst: Die beiden Messkurven unterscheiden sich 
innerhalb des Signal-Rausch-Verhältnisses nicht voneinander, wenn der Pumpstrahl ein- und ausgeschaltet wird. Eine geeignete Wahl der Zeitabstände $t_{12}$ und $t_{23}$ der eingestrahlten Laserpulse stellt sicher, dass kein Signal in der Detektionsrichtung des HeNe-Laserstrahls erzeugt wird.

Die hier aufgenommenen Änderungen der optischen Dichte bei $632 \mathrm{~nm}$ als Funktion der Temperatur sind mit unabhängig hiervon durchgeführten temperaturabhängigen Messungen des stationären Absorptionsspektrums von $\mathrm{DiDC}_{18}$ in DMPC (Abbildung 5.10 blaue Kurve) konsistent. Zwischen $15^{\circ} \mathrm{C}$ und $27^{\circ} \mathrm{C}$ ergibt sich sogar eine gute Übereinstimmung.

\subsection{Farbstoffsynthese}

Für die Messungen wurden kommerziell erhältliche Farbstoffe und Lipide eingesetzt. Lediglich das 1,1'-Dioctadecyl-3,3,3',3'-tetramethylindodicarbocyaninchlorid ( DiC $\left._{18}\right)$ wurde synthetisiert. Für die Herstellung waren drei Syntheseschritte notwendig (Abbildung 5.11). Edukte waren das p-Chlorobenzolsulfochlorid(1b) und der Stearylalkohol (1a). In der ersten Stufe wurde das Alkylierungsreagenz 4-Chlorobenzolsulfonsäure-octadecylester (2a) hergestellt. 2,3,3-Trimethyl-3H-indol (2b) wurde anschließend mit dem Produkt (2a) aus der ersten Reaktion alkyliert. Das so erhaltene 2,3,3-Trimethyl-1-octadecyl-3H-indolium-4-chlorbenzoat (3a) wurde durch eine Reaktion mit 3-Anilinoacrylaldehydanil (3b) zum DiDC $_{18}$ verbrückt. Eine detaillierte Versuchsdurchführung ist dem Anhang zu entnehmen. 


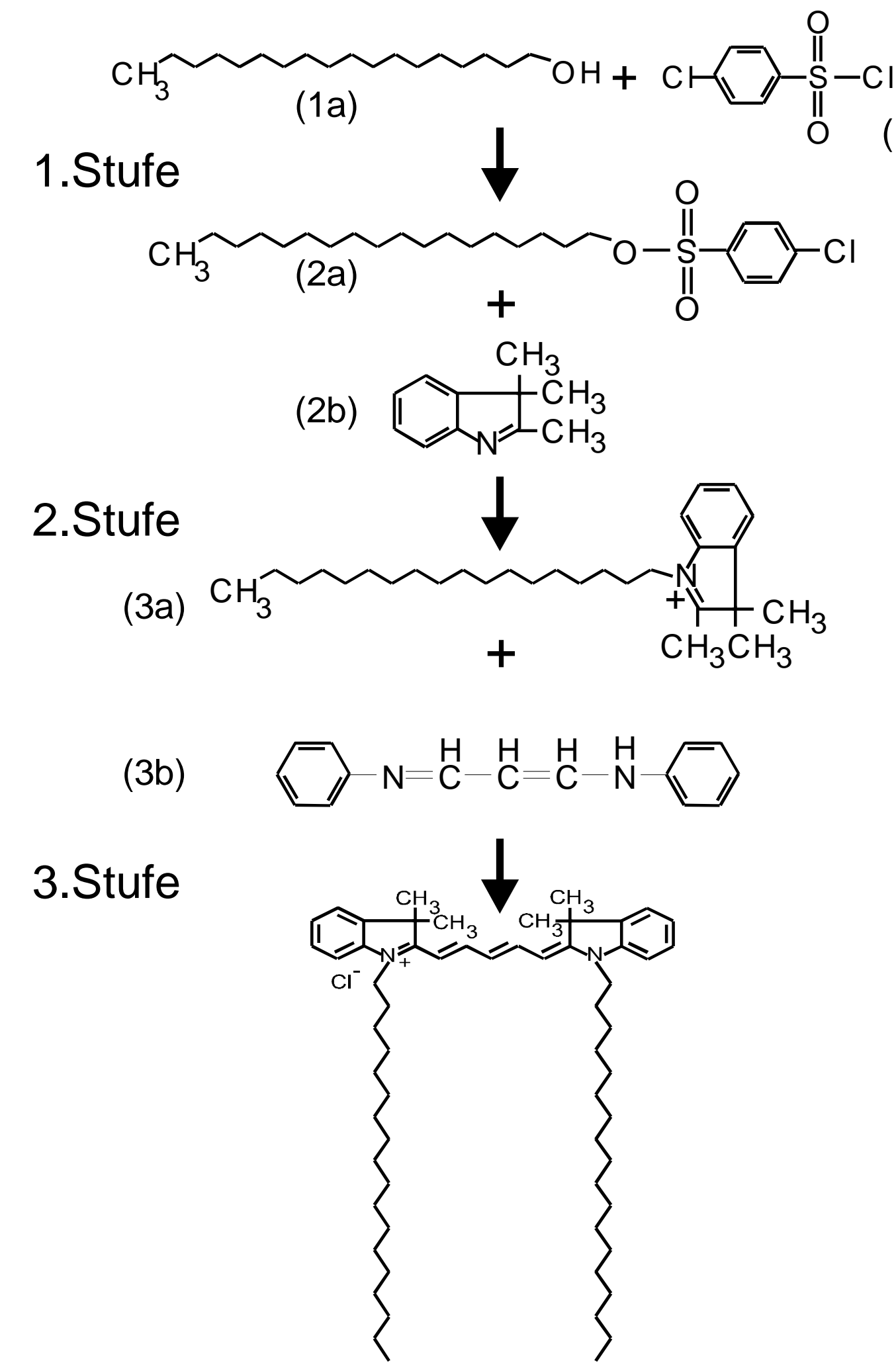

1.Stufe

(2b)

2.Stufe

(3a)

Abbildung 5.11: Schematische Darstellung der dreistufigen Synthese von

1,1'-Dioctadecyl-3,3,3',3'-tetramethylindodicarbocyaninchlorid 


\subsection{Probenmoleküle und Vesikelpräparation}

Im Verlauf der Untersuchungen wurden zwei Farbstoffmoleküle und drei unterschiedliche Lipide eingesetzt. Als Lösungsmittel wurde Wasser verwendet.

\begin{tabular}{|l|l|l|}
\hline Substanz & Hersteller/Lieferant & Reinheitsgrad \\
\hline DMPC & Avanti, Fluka & $>99 \%$ \\
\hline DOPC & Avanti & $>99 \%$ \\
\hline DPPC & Avanti & $>99 \%$ \\
\hline Wasser & Merck & zweifach destilliert \\
\hline DiDC $_{1}$ & Lambda Physik & ohne Angabe \\
\hline DiDC $_{18}$ & eigene Synthese & mehrfach umkristallisiert \\
\hline
\end{tabular}

Tabelle 5.1

Die gekauften Substanzen wurden ohne weitere Reinigung eingesetzt. Das synthetisierte $D_{i D C}{ }_{18}$ wurde mehrfach umkristallisiert und in einer Kieselgelsäule gereinigt. Die Hersteller und Reinheitsgrade sind soweit bekannt in Tabelle 5.1 angegeben.

\subsubsection{Farbstoffe}

Der Chromophor der Farbstoffe besteht in beiden Fällen aus einer Indodicarbocyanineinheit. In Abhängigkeit der zu untersuchenden Zielumgebung bestehen die Alkylgruppen am Stickstoff aus kurzen oder langen aliphatischen Ketten. Für die Untersuchungen der Membrangrenzschicht wird eine lange gesättigte Alkylkette mit 18 Kohlenstoffatomen verwendet. Der so erhaltene Farbstoff 1,1'Dioctadecyl-3,3,3',3'-tetramethylindodicarbocyaninchlorid $\left(\mathrm{DiDC}_{18}\right)$ ist aufgrund der langen aliphatischen Kette mit ihrem unpolaren Charakter wasserunlöslich. Die Ketten des Farbstoffs dienen als Anker für die Membrandoppelschicht. Der Chromophor befindet sich in diesem Fall direkt an der Grenzschicht, wie Fluoreszenz-Polarisations-Messungen $[115,116]$ gezeigt haben. Des Weiteren haben Untersuchungen gezeigt, dass dieser Farbstoff die nach außen gerichtete Seite der Doppelschicht der Vesikel bevorzugt [117]. 
Beim zweiten eingesetzten Farbstoff ist eine Methylgruppe an das Stickstoffatom gebunden. Das 1,1',3,3,3',3'-Hexamethylindodicarbocyaniniodid (DiDC ${ }_{1}$ oder HIDCl) ist daher wasserlöslich und wird bei den Photon-Echo-Experimenten in reinem Wasser verwendet.

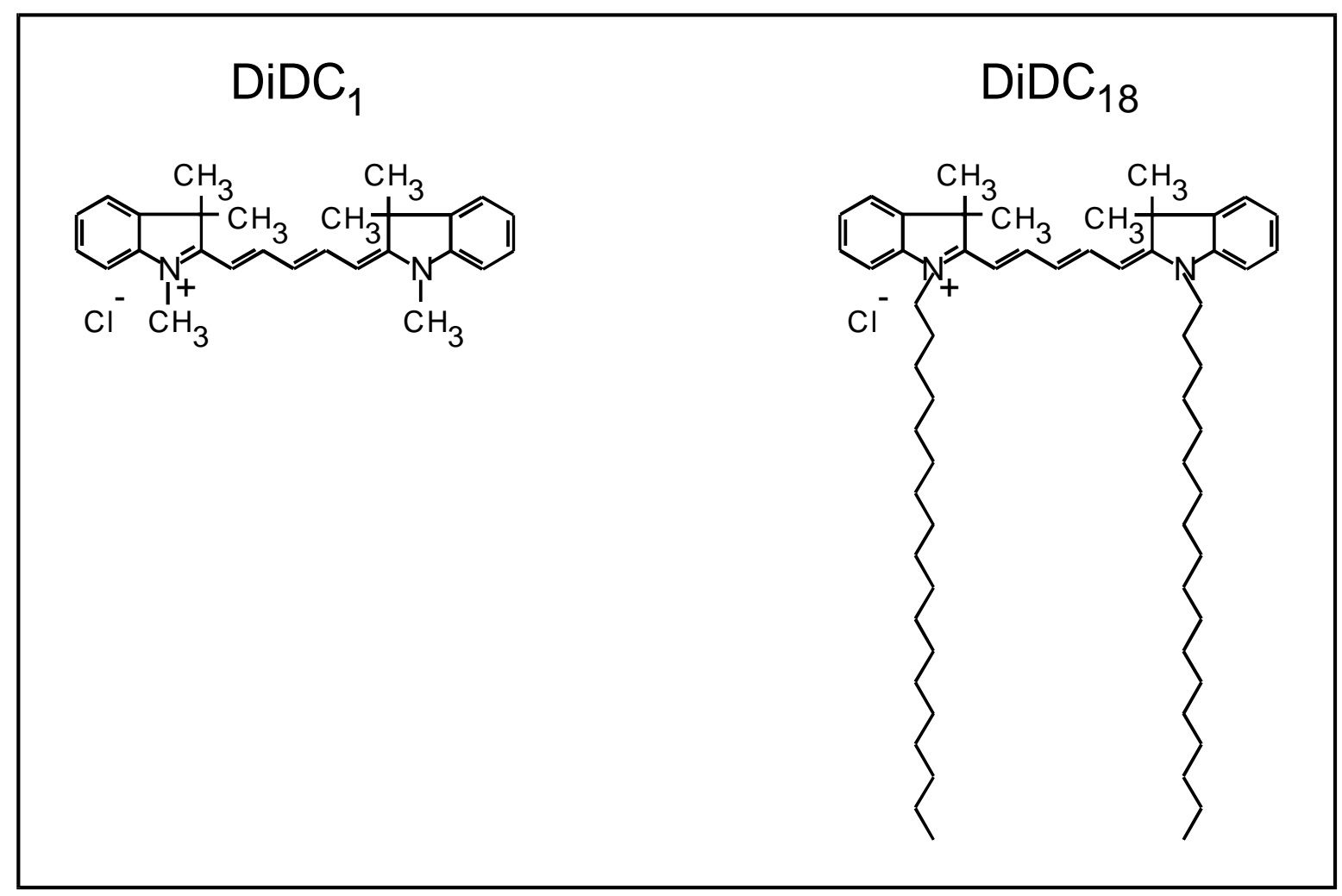

Abbildung 5.12: Indodicarbocyanine DiDC $_{1}$ und DiDC $_{18}$

Die stationären Absorptions- und Emissionsspektren beider Farbstoffe werden in Kapitel 6.1 gezeigt und diskutiert. Die Absorptionsspektren wurden mit einem Shimadzu UV-160 Spektrometer gemessen. Die Fluoreszenzspektren wurden dagegen mit einem Spex Fluorolog III der Firma Jobin-Yvon bestimmt.

\subsubsection{Lipide}

Die eingesetzten Lipide gehören zu den in der Natur vorkommenden Lipiden und dienen daher in reiner Form als Modellsysteme. Sie gehören der großen Gruppe der 1,2-Diacyl-sn-Glycero-Phosphocholine an. Mit ihren zwei Alkylketten und der polaren Kopfgruppe ähneln sie in ihrer Polarität dem $\operatorname{DiDC}_{18}$. Die verwendeten Lipide besitzen gesättigte Alkylketten mit 14 (1,2-Dimyristoyl-sn-Glycero-Phosphocholine; 
DMPC) und 16 (1,2-Dipalmitoyl-sn-Glycero-Phosphocholine; DPPC) Kohlenstoffatomen. Das dritte Lipid dagegen besitzt eine cis-Doppelbindung am 9. Kohlenstoffatom bei insgesamt 18 Kohlenstoffatomen (1,2-Dioleoyl-sn-GlyceroPhosphocholine; DOPC). Alle Lipide haben eine identische PhosphocholinKopfgruppe.

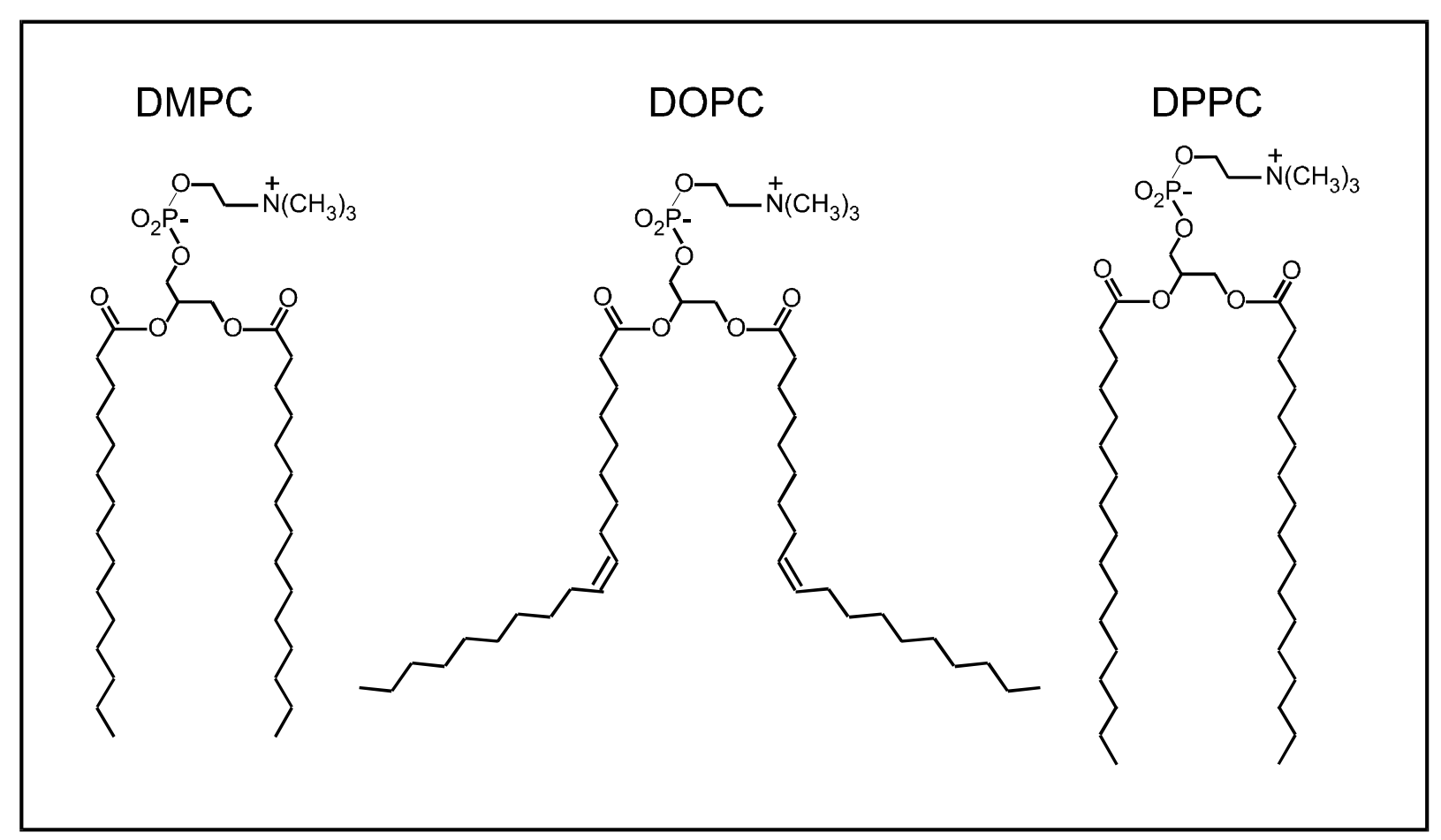

Abbildung 5.13: Glycero-phosphocholine: DMPC, DOPC, DPPC

\subsubsection{Vesikelherstellung}

In den Photon-Echo-Experimente wurden Lösungen mit einer Lipidkonzentration von $20 \mathrm{mmol} / \mathrm{l}$ bzw. $10 \mathrm{mmol} / \mathrm{l}$ verwendet und mit $\mathrm{DiDC}_{18}$ markiert. In diesen Lösungen besteht ein Verhältnis zwischen den Lipiden und dem Farbstofff DiDC $_{18}$ von 1000:1 bzw. 500:1.

Die Herstellung der mit Farbstoff markierten Vesikel erfolgte in mehreren Schritten. In einem kleinen Becherglas wird zunächst die notwendige Menge Lipid eingewogen. Anschließend wird eine Dichlormethanlösung des Farbstoffs DiDC $_{18}$ mit bekannter Konzentration hinzugefügt. Das so erhaltene Gemisch wird bis zum vollständigen Lösen der Lipide geschüttelt. Für DPPC und DOPC ist zum Lösen eine Zugabe von geringen Mengen Methanol erforderlich. Nun wird das Lösungsmittel unter einer Stickstoffatmosphäre verdampft. Zur vollständigen Beseitigung von 
Lösungsmittelresten wird das Becherglas mit der Substanz über einen Zeitraum von 4 - 6 Stunden in einem Exsikkator aufbewahrt. Der Exsikkator wird während dieser Zeit an eine Ölpumpe angeschlossen. Die mit $\mathrm{DiDC}_{18}$ markierten Lipide werden anschließend mit Wasser versetzt und mit einem Magnetrührer gerührt bis sich eine gleichmäßige Dispersion bildet. Diese Dispersion wird extrudiert. Der eingesetzte Liposo-Fast Extruder von der Firma Avestin, Inc. besteht aus zwei Glasspritzen und einem Metallzwischenstück, welches zwei Plastikteile zusammenhält. Die Plastikteile dienen der Fixierung eines Polycarbonatfilters.

Extrudiert wird, indem eine Spritze mit Substanz gefüllt wird. Beide Spritzen werden an die Plastikfilterhalterungen angeschraubt und die Emulsion wird durch die Membran von einer Spritze in die andere gepresst. Dieser Vorgang wird 19 mal wiederholt. Die so erhaltene Lösung enthält Vesikel in einer von der Porengröße des Filters abhängigen Größenverteilung. Es wurden 100 nm Filter eingesetzt. Die resultierende Größenverteilung ist damit bei $80 \mathrm{~nm}$ zentriert [17].

Bevor die präparierten Lösungen in den Experimenten eingesetzt werden, wird die gelöste Luft durch ein schwaches Vakuum beseitig. Die so gewonnene Probe ist nur einige Wochen stabil. Aus diesem Grund wurde direkt vor der Messung extrudiert. 


\section{ERGEBNISSE UND DISKUSSION}

Zentraler Gegenstand der vorliegenden Arbeit ist die Bestimmung der Zeitskalen und molekularen Mechanismen der dynamischen Solvatation an Phospholipid-WasserGrenzschichten.

Als Sonde für die dynamische Solvatation in diesem System wird ein Indocarbocyaninfarbstoff verwendet, dessen Chromophor an der Wasser-Lipid-Grenzschicht lokalisiert und über langkettige Alkylreste im hydrophoben Kern der Membran nichtkovalent verankert ist (siehe Abbildung 6.1) [115-117].

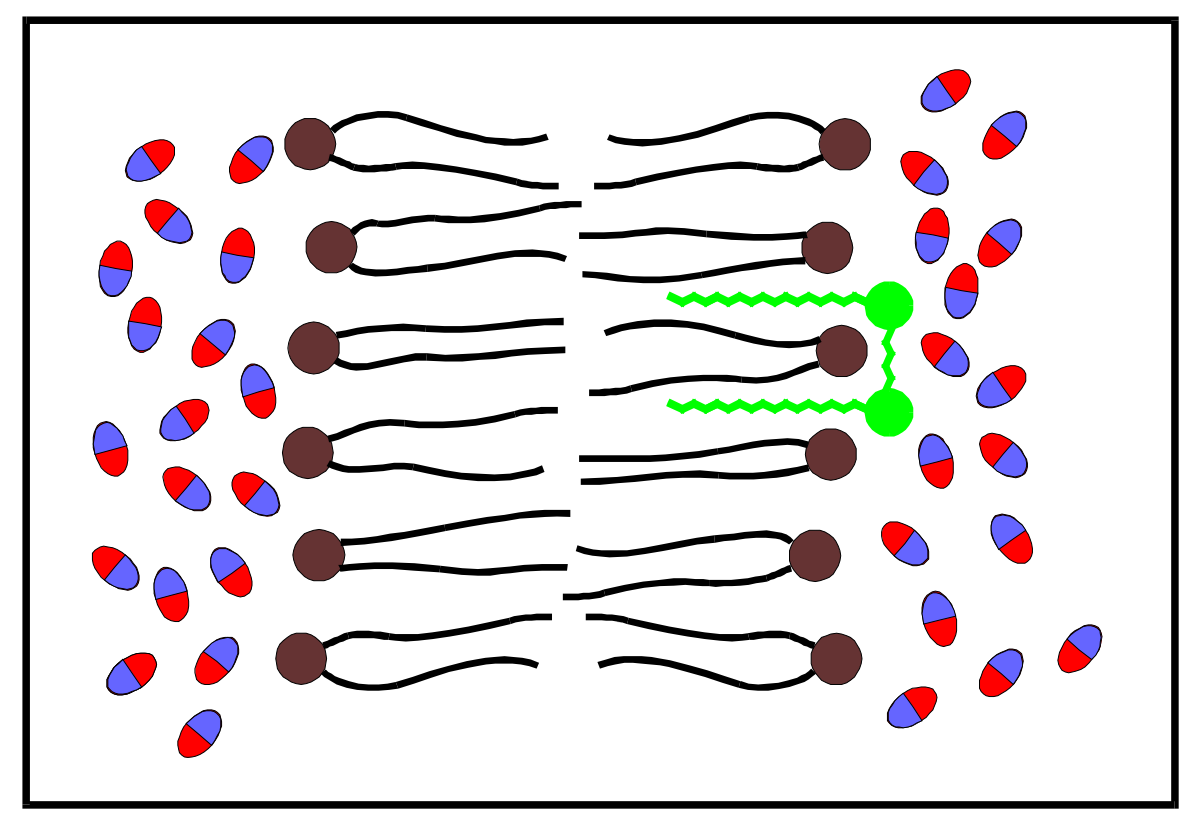

Abbildung 6.1: Schematische Abbildung einer Lipiddoppelschicht (schwarz) mit dem SensorFarbstoff DiDC $_{18}$ (grün) und umgebenden Wasserdipolen (rot/blau)

Für eine detaillierte Analyse der Solvatation an Phospholipid-Wasser-Grenzschichten ist es wichtig, zwischen grenzschicht-spezifischen Dynamiken und denen der wässrigen Volumenphase unterscheiden zu können. Aus diesem Grund wird zunächst die Solvatationsdynamik des Chromophors in reinem Wasser (also in Abwesenheit der Lipid-Grenzschicht) ermittelt.

In der wässrigen Volumenphase dient wiederum der Indocarbocyaninfarbstoff, dessen langkettige Alkylreste durch Methylgruppen substituiert sind, als Sonde für Solvatationsdynamiken. Diese einfache Substitution verbessert die Wasserlöslichkeit des ansonsten identischen Chromophors. 


\subsection{Allgemeine Untersuchungen der Farbstoffe und der Lipidlösungen}

\section{a) Stationäre Absorptions- und Emissionsspektren}

In Abbildung 6.2 sind die stationären Absorptions- und Emissionsspektren der verwendeten Cyaninfarbstoffe abgebildet. Die Spektren der Farbstoffe in reinem Wasser und an der DMPC-Wasser-Grenzschicht unterscheiden sich nur geringfügig. Im Vergleich zu Wasser sind die Spektren von $\mathrm{DiDC}_{18}$ an der Lipidmembran-WasserGrenzschicht um $266 \mathrm{~cm}^{-1}$ zu niedrigeren Wellenzahlen verschoben. Diese Rotverschiebung lässt sich durch die im Vergleich zum reinem Wasser höhere lokale Dichte an der Grenzschicht erklären.

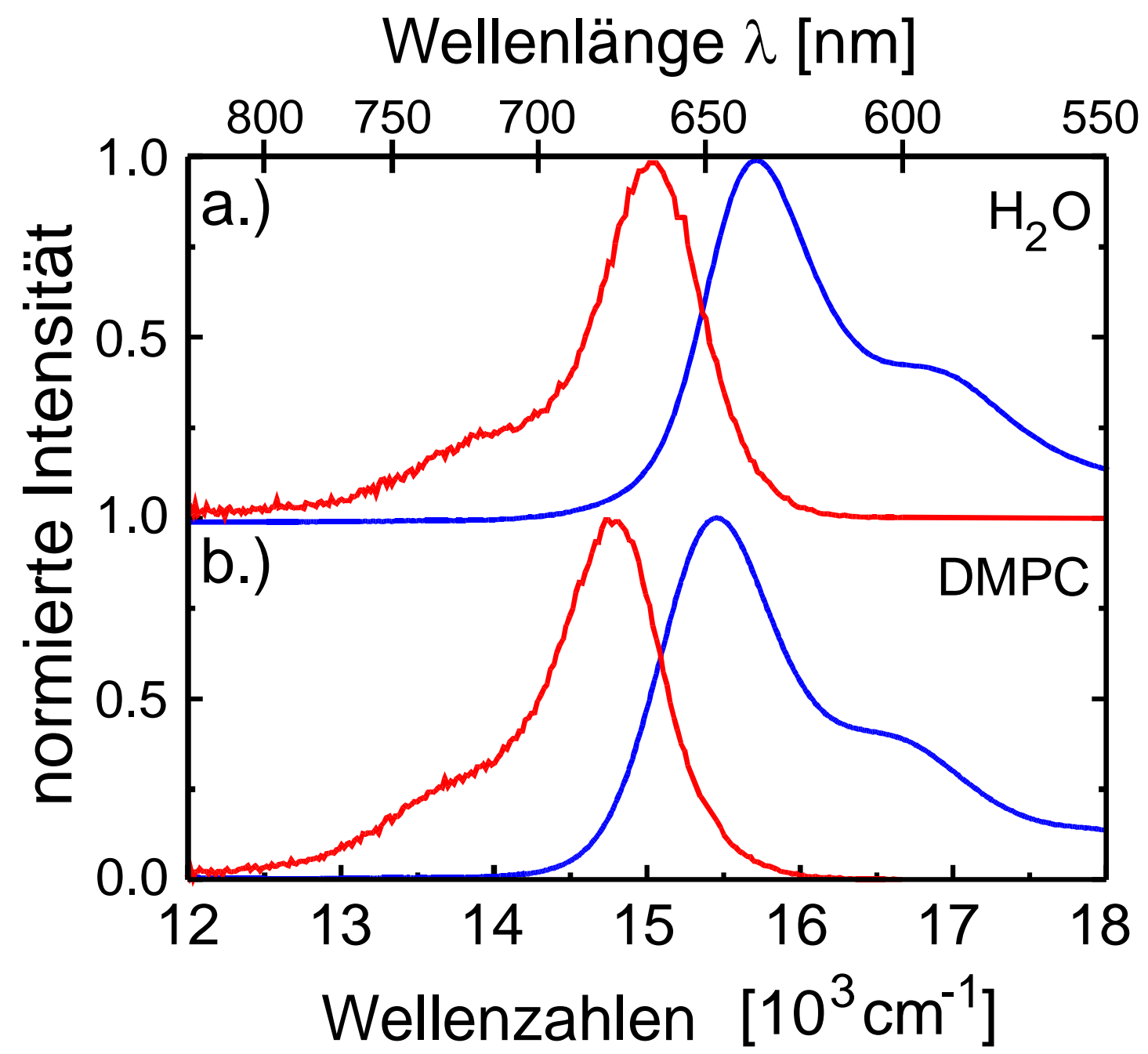

Abbildung 6.2: Stationäre Absorptions- (blau) und Emissionsspektren (rot) aufgetragen gegen Wellenzahlen in reziproken Zentimetern bzw. der Wellenlänge $\lambda$ in $\mathbf{n m :}$ a.) DiDC $_{1}$ in Wasser, b.) DiDC 18 in DMPC/Wasser. 
Die Form der Absorptions- und Emissionsspektren ändert sich dagegen nicht und verdeutlicht, dass das elektronische Übergangsmoment ausschließlich auf dem Indocarbocyaninchromophor ruht und nicht wesentlich von den Alkylsubstituenten beeinflusst wird. Selbst die Stokes-Verschiebung von $685 \mathrm{~cm}^{-1}$ ist für beide Farbstoffe gleich. Dies weist auf eine identische Reorganisationsenergie hin.

Ein Charakteristikum der Indocarbocyaninfarbstoffe ist die ausgeprägte Neigung zur Aggregatbildung, die die optischen Eigenschaften des Farbstoffes in dramatischer Weise beeinflusst $[147,148]$. Die sich bildenden Aggregate werden in der Literatur J-Aggregate genannt. Diese Oligomere zeigen im Vergleich zum Monomer ein stark rotverschobenes, schmales Absorptionsspektrum, welches für eine exzitonische Kopplung im Grenzfall des „motional narrowings“ des J-Aggregates typisch ist [120]. Bei tiefen Temperaturen macht sich neben einer schmalen Absorptionsbande der J-Aggregate eine verkürzte Fluoreszenzlebensdauer bemerkbar, die ihre Ursache in kooperativer Emission („superradiance“) hat. Diese Abstrahlung steht in direkter Beziehung zur Anzahl der miteinander koppelnden Monomere [121].

Die oben erwähnten Eigenschaften solcher J-Aggregate konnten bei den durchgeführten Experimenten in Wasser und an der Lipidmembran-WasserGrenzschicht nicht beobachtet werden. Die Formstabilität der Spektren und die Abwesenheit einer rotverschobenen Exzitonenresonanz im Speziellen belegt eine vernachlässigbare Aggregation.

\section{b) Kalorimetrische Messungen der Phasenübergänge der reinen und der markierten Vesikel}

In Abbildung 6.3 sind die Wärmekapazitäten der Vesikellösungen von DMPC und DPPC gegen die Temperatur $T$ aufgetragen. Neben den reinen Lipidlösungen (schwarze Kurven) sind auch die Wärmekapazitätsmessungen von den mit $\mathrm{DiDC}_{18}$ markierten Vesikellösungen abgebildet (rote Kurven). Anhand der Wärmekapazität sind die Phasenübergangstemperaturen des Vor- und Hauptphasenübergangs leicht zu bestimmen. Die Temperatur für den Hauptphasenübergang wird anhand des bei der höheren Temperatur gelegenen Maximums der Wärmekapazität ermittelt. Der Vorphasenübergang befindet sich bei deutlich tieferen Temperaturen und ist daher lediglich in der Einblendung des jeweiligen Graphen (Abbildung 6.3) dargestellt. Seine Temperatur entspricht der Temperatur am Wendepunkt der Wärmekapazität in diesem Bereich. Bei unmarkiertem DMPC ergibt sich eine Vorphasenübergangstemperatur von $11.3^{\circ} \mathrm{C}$. Nach Markierung mit dem Farbstoff liegt der Übergang bei 
$10.8^{\circ} \mathrm{C}$. Der Hauptphasenübergang findet dagegen bei einer Temperatur von $23.9^{\circ} \mathrm{C}$ (unmarkiertes DMPC) bzw. $23.7^{\circ} \mathrm{C}$ (markiertes DMPC) statt.

Bei DPPC verschieben sich die Phasenübergänge auf Grund der längeren aliphatischen Ketten zu höheren Temperaturen. Die Messungen ergeben Phasenübergangstemperaturen von $33.0^{\circ} \mathrm{C}$ und $41.2^{\circ} \mathrm{C}$ für unmarkiertes DPPC sowie $32.3^{\circ} \mathrm{C}$ und $41.1^{\circ} \mathrm{C}$ für markiertes DPPC. Diese Temperaturen entsprechen den in der Literatur angegebenen Werten [122].

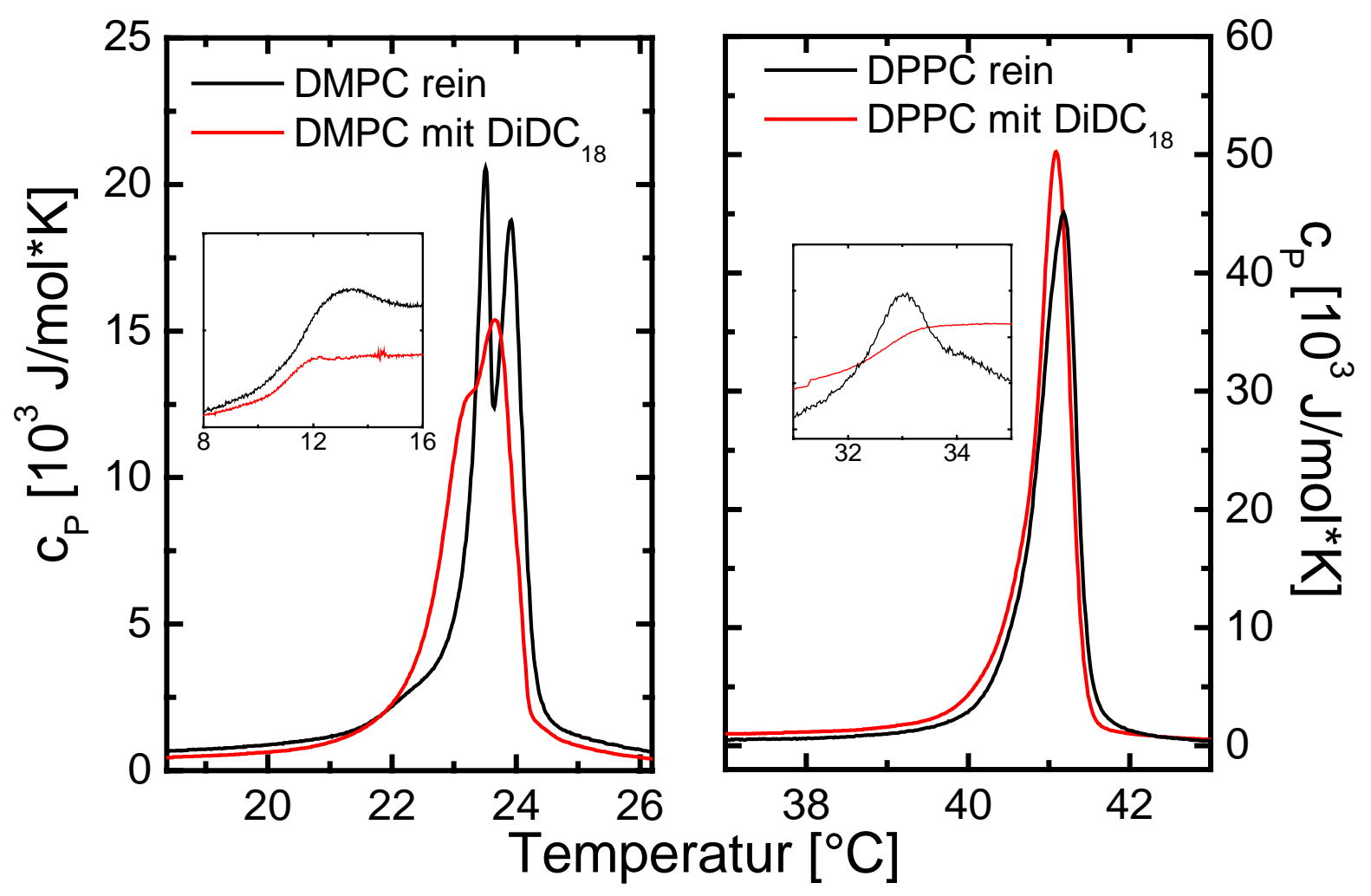

Abbildung 6.3: Messungen der Wärmekapazität $c_{p}$ als Funktion der Temperatur im Bereich der Phasenübergänge von DMPC- und DPPC-Vesikeln mit (rot) und ohne (schwarz) DiDC $_{18}$ Marker

Eine Wärmekapazitätsmessung unilamellarer Vesikel von DOPC liefert eine Hauptphasenübergangstemperatur von $-18^{\circ} \mathrm{C}$ (nicht abgebildet).

Die Veränderung der Kurvenform der Wärmekapazität von Lipidmembranen durch den Farbstoff lässt keine eindeutige Interpretation zu, da bislang unklar ist, woher beispielsweise die zwei Maxima beim Hauptphasenübergang herrührt. Vermutet wird ein struktureller Übergang in den Vesikeln [123]. Fremdmoleküle könnten diesen Übergang beschleunigen und die Maxima zu einem einzelnen Maximum verschmelzen lassen. 
Die Position des Phasenübergangs ist dagegen von größerer Bedeutung. Eine Verschiebung des Hauptphasenübergangs in Abhängigkeit des Farbstoffes ist bekannt und wird mit einer Bevorzugung des Farbstoffes für eine der beiden Phasen erklärt. Ein zu tieferen (höheren) Temperaturen verschobener Hauptphasenübergang ist bei Farbstoffen zu beobachten, deren Löslichkeit in der gelförmigen (flüssigkristallinen) Phase höher ist als in der flüssig-kristallinen (gelförmigen) Phase [124126]. Insgesamt werden die Phasenübergangstemperaturen der Vesikel durch den Farbstoff $\mathrm{DiDC}_{18}$ nur unwesentlich verändert.

\section{c) Temperaturabhängige Messungen der optischen Dichte}

Die Absorptionsspektren von $\mathrm{DiDC}_{1}$ in reinem Wasser (siehe Abbildung 6.4) verändern sich durch Variation der Temperaturen nur wenig. Das Verhalten der stationären Absorptionsspektren bei steigender Temperatur ist mit Pfeilen angedeutet. Während sich die Zunahme der optischen Dichte an der roten Flanke des Absorptionsspektrums durch thermische Anregung erklären lässt, ist die genaue Ursache für die Abnahme der optischen Dichte in den beiden anderen markierten Bereichen nicht bekannt.

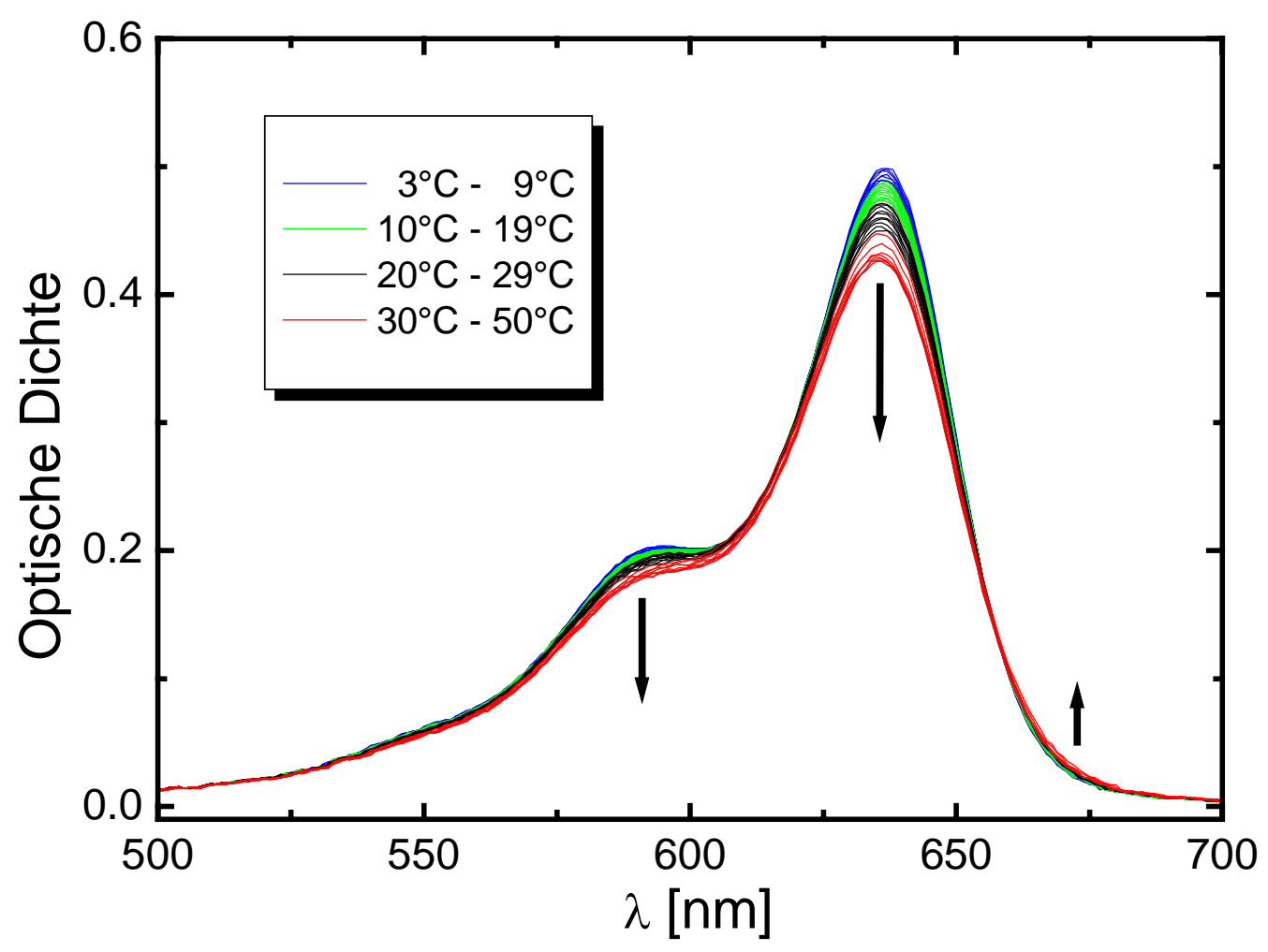

Abbildung 6.4: Absorptionsspektren von $\mathrm{DiDC}_{1}$ in reinem Wasser in Abhängigkeit der Temperatur im Bereich von $3^{\circ} \mathrm{C}-50^{\circ} \mathrm{C}$ 
Im Gegensatz zum Farbstoff zeigt das Absorptionsspektrum der reinen DMPC- und DPPC-Vesikel eine deutliche Temperaturabhängigkeit (siehe Abbildung 6.5). Beide Lösungen wurden mit identischer Konzentration von $20 \mathrm{mmol} / \mathrm{l}$ hergestellt. Die Porengröße des Extrudierfilters war $100 \mathrm{~nm}$ und die Schichtdicke der Messzelle betrug $1 \mathrm{~mm}$. Trotz identischer Präparation beider Lösungen unterscheiden sich die Messergebnisse der beiden Proben deutlich voneinander.
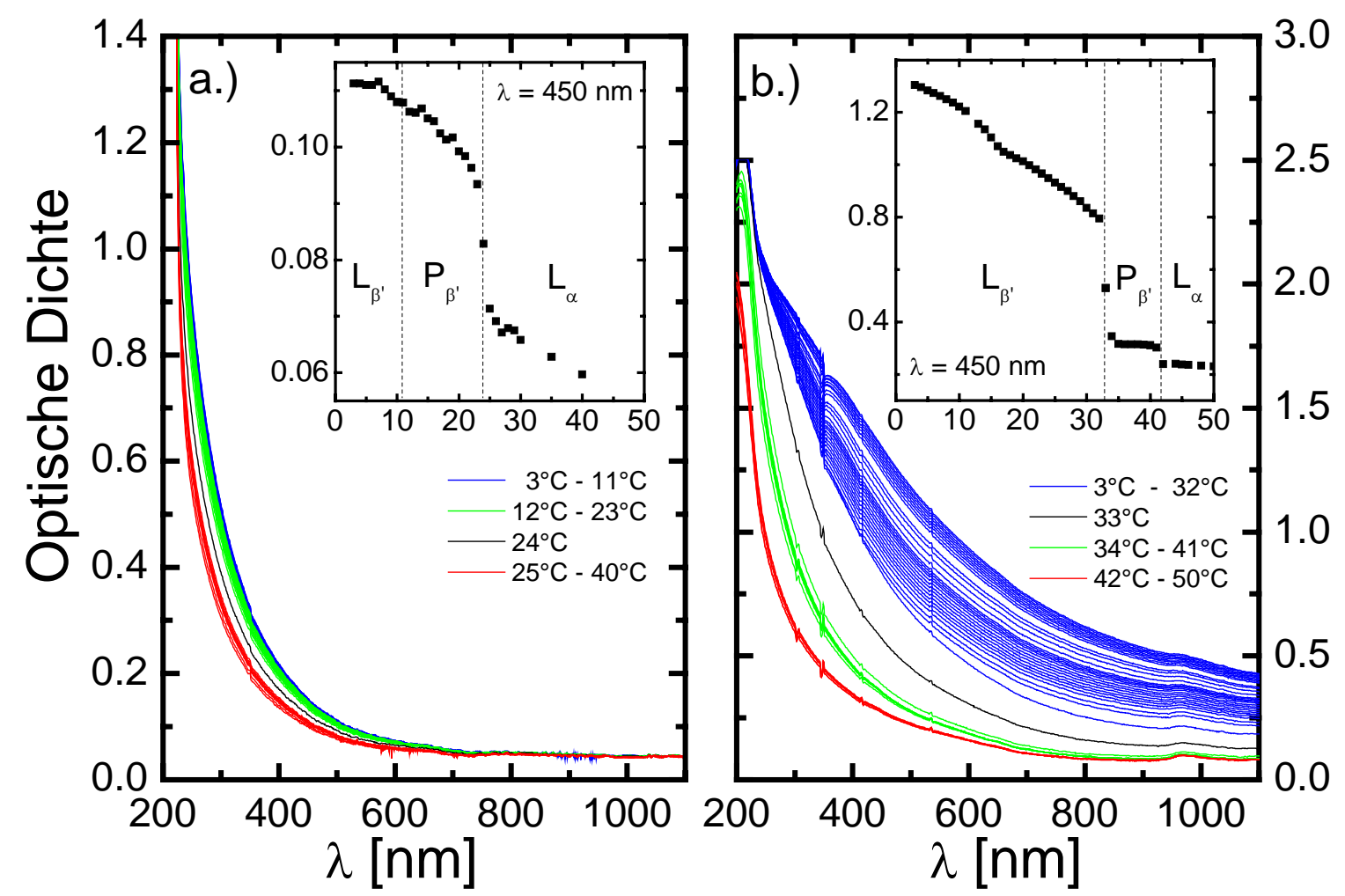

Abbildung 6.5: Temperaturabhängige Messungen der optische Dichte von extrudierten

a.) DMPC- b.) DPPC-Vesikeln mit einer Konzentration von 20 mmol/l:

(blau: gelförmige Phase; grün: zackige Phase; rot: flüssig-kristalline Phase)

Die optische Dichte beider Lipidlösungen nimmt zu kürzeren Wellenlängen stark zu und sinkt mit steigender Temperatur.

Die in Abbildung 6.5 a.) dargestellten Spektren der DMPC-Vesikeln zeigen eine im Vergleich zu den DPPC-Vesikeln geringere Temperaturabhängigkeit. Die Zunahme der optischen Dichte beträgt im Wellenlängenbereich von $1100 \mathrm{~nm}$ bis $400 \mathrm{~nm}$ maximal 0.1. In der Einblendung ist die Absorption bei einer festen Wellenlänge von $\lambda=450 \mathrm{~nm}$ gegen die Temperatur aufgetragen. Bei der Temperatur des Hauptphasenübergangs $\left(P_{\beta}, \rightarrow L_{\alpha}\right)$ ist ein deutlicher Sprung zu beobachten, während bei 
der Temperatur des Vorphasenübergangs $\left(L_{\beta}, \rightarrow P_{\beta}\right)$ praktisch keine Änderung der Absorption zu erkennen ist.

Im Gegensatz zu DMPC-Vesikeln ist bei DPPC-Vesikeln eine Zunahme der optischen Dichte über den gesamten Spektralbereich zu beobachten. Sowohl der Vorphasenübergang als auch der Hauptphasenübergang der Vesikel ist mit einer verstärkten Abnahme der optischen Dichte bei Erhöhung der Temperatur verbunden (Abbildung 6.5 b.)). Die Veränderung der Absorption am Vorphasenübergang ist bei DPPC-Vesikeln im Gegensatz zu DMPC-Vesikeln besonders ausgeprägt.

Für das auffällige Temperaturverhalten der optischen Dichte der Vesikel, können verschiedene Erklärungsmodelle herangezogen werden. Im Falle einer elektronischen Absorption der Lipide gilt die folgende lineare Abhängigkeit des LambertBeerschen Gesetzes:

$$
\mathrm{OD}=-\log \frac{\mathrm{I}}{\mathrm{I}_{0}} \propto \text { Konzentration }, \quad \text { Lambert-Beersches Gesetz }
$$

\section{Gleichung 6.1}

mit $I_{0}$ als eingestrahlte und $I$ als transmittierte Lichtintensität. Bei Gültigkeit dieses Zusammenhangs würde eine Auftragung der optischen Dichte gegen die Konzentration eine lineare Abhängigkeit aufweisen.

Da die Vesikel mit einem Durchmesser von ca. $100 \mathrm{~nm}$ eine mit der Wellenlänge des eingestrahlten Lichtes vergleichbare Größe aufweisen, könnte das auffällige Verhalten der optischen Dichte alternativ durch Mie-Streuung verursacht werden. In diesem Fall gilt der folgende Zusammenhang [127]:

$$
\frac{\mathrm{I}_{0}-\mathrm{I}}{\mathrm{I}_{0}}=-\sigma \cdot \mathrm{n}_{0} \cdot \mathrm{dx} \quad \rightarrow \quad \frac{\mathrm{I}_{0}-\mathrm{I}}{\mathrm{I}_{0}} \propto \text { Konzentration } \quad \text { Mie-Streuung }
$$

\section{Gleichung 6.2}

mit $I_{0}$ als eingestrahlte und $I$ als transmittierte Lichtintensität. Die Parameter $\sigma$ und $n_{0}$ beschreiben den Streuquerschnitt der Teilchen bzw. deren Anzahldichte (Konzentration). Der in der Lösung vom Licht zurückgelegte Weg wird mit dx bezeichnet. 
Um zwischen diesen beiden Modellen unterscheiden zu können, wurden konzentrationsabhängige Messungen an DPPC-Vesikeln durchgeführt. Dabei betrug die Temperatur $24^{\circ} \mathrm{C}$, während die Konzentration von $5 \mathrm{mmol} / \mathrm{l}$ bis $20 \mathrm{mmol} / /$ variiert wurde. In Abbildung 6.6 a.) sind die entsprechenden Ergebnisse dargestellt, wobei farbstoffmarkierte Vesikellösungen verwendet wurden. Die Absorptionsbande zwischen $500 \mathrm{~nm}$ und $750 \mathrm{~nm}$ ist daher der elektronischen Resonanz des Indocarbocyaninfarbstoffes zuzuschreiben.
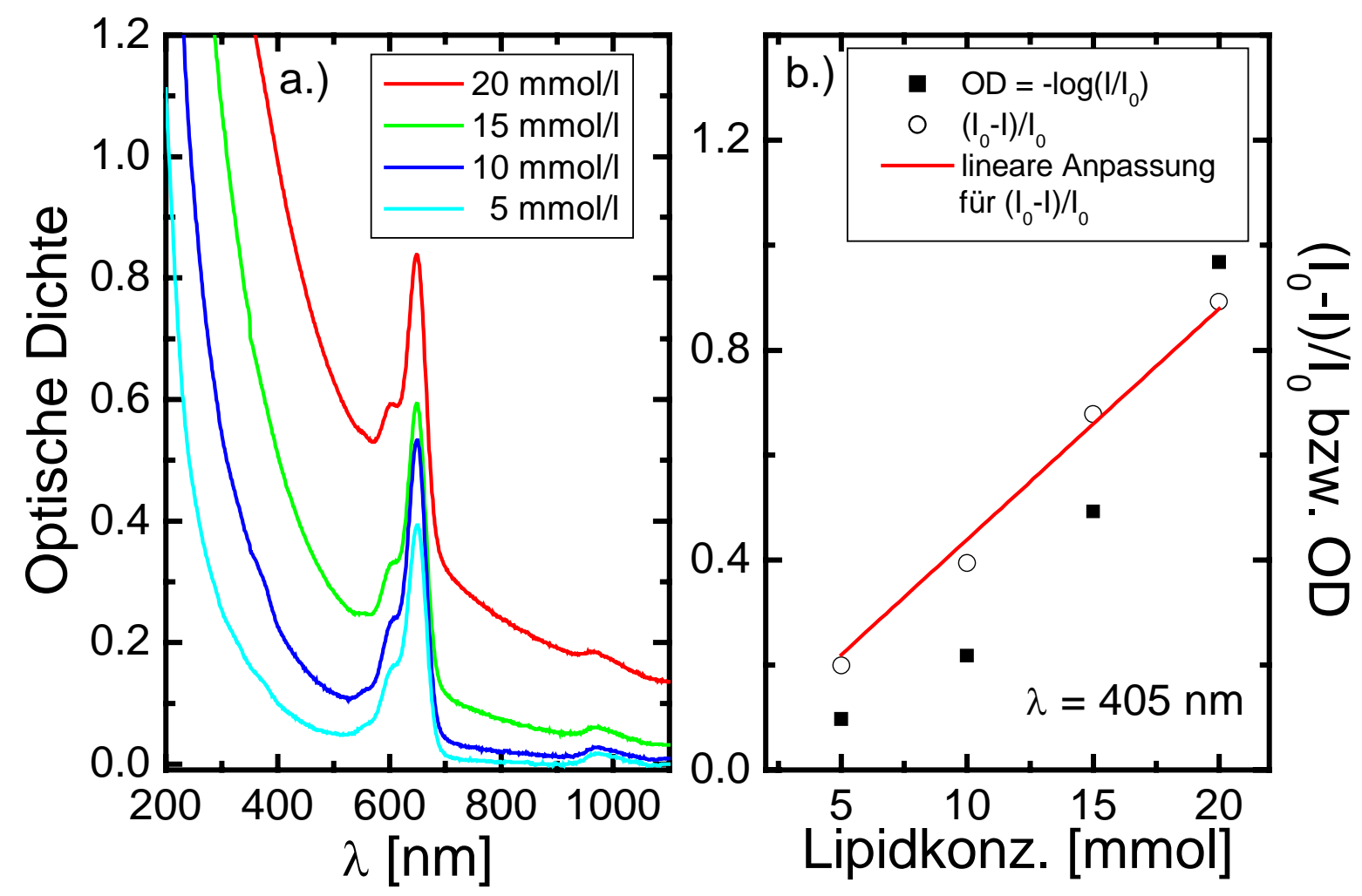

Abbildung 6.6: Konzentrationsabhängige Messung der Absorption von DPPC-Vesikeln bei einer Temperatur von $24^{\circ} \mathrm{C}$ :

a.) Absorptionsspektren von DPPC (mit DiDC ${ }_{18}$ markiert)

b.) Auftragung der optischen Dichte (OD) bzw. des Verhältnisses $\left(I_{0}-1\right) / I_{0}$ gegen die Lipidkonzentration bei $\lambda=405 \mathrm{~nm}$

Eine Erhöhung der Vesikelkonzentration äußert sich in einer signifikanten Vergrößerung der optischen Dichte über den gesamten Wellenlängenbereich. Zur Unterscheidung der beiden Erklärungsmöglichkeiten ist in Abbildung 6.6 b.) die optische Dichte (Absorption) sowie das Verhältnis $\left(I_{0}-1\right) / I_{0}$ (Streuung) als Funktion der Lipidkonzentration bei $\lambda=405 \mathrm{~nm}$ aufgetragen. Die Auftragung verdeutlicht, dass im 
Gegensatz zur optischen Dichte die Größe $\left(I_{0}-I\right) / I_{0}$ linear mit der Konzentration skaliert (Gerade in Abbildung 6.6 b.)). Es handelt sich bei dem beobachteten temperaturabhängigen Verhalten der optischen Dichte offensichtlich um MieStreuung.

Ein weiteres Indiz für Mie-Streuung ist das veränderte Spektrum der Vesikel, das sich bei der Benutzung eines Filters mit $30 \mathrm{~nm}$ Porendurchmesser bei ihrer Herstellung ergibt (ohne Abbildung). Die mit diesem Filter extrudierten Lösungen weisen ein in den ultravioletten Spektralbereich verschobenes Spektrum auf und der Untergrund im sichtbaren Spektralbereich nimmt ab.

Im Folgenden soll mit Hilfe der gewonnenen Erkenntnis, dass es sich um MieStreuung handelt, das temperaturabhängige Verhalten der optischen Dichte der Vesikel erläutert werden. Nach dem Lichstreuungsgesetz, bedeutet eine zunehmende optische Dichte bei gleichbleibender Konzentration $\mathrm{n}_{0}$ und Weglänge $\mathrm{dx}$ eine Änderung des Streuquerschnitts $\sigma$. Eine einfache Erklärung dieser Temperaturabhängigkeit ist die Zunahme der Vesikelgröße, für die zwei Mechanismen in Frage kommen:

Eine Möglichkeit besteht in der Fusion von Vesikeln bei der sich zwei Vesikel irreversibel zu einem Vesikel zusammenschließen. Die in dieser Arbeit beobachtete Änderung der optischen Dichte mit der Temperatur ist allerdings reversibel, da sie beim Abkühlen als auch beim Erwärmen auftritt. Eine weitere Erklärungsmöglichkeit besteht in der Aggregation von Vesikeln. Dabei stoßen zwei Vesikel zusammen und bleiben aneinander haften. Dieses Vesikelaggregat streut dementsprechend langwelligeres Licht stärker. In der Literatur ist dieser Prozess der Aggregatsbildung von Vesikeln als Adhäsion bekannt [128].

Die Markierung der Vesikel mit $\mathrm{DiDC}_{18}$ führt zu den folgenden Beobachtungen. Die Maxima des Absorptionsspektrums des Farbstoffes in DMPC- und DPPC-Vesikeln verschieben sich mit zunehmender Temperatur zu höheren Frequenzen (Abbildung 6.7). Bei Überschreiten der Temperatur des Hauptphasenübergangs verschiebt sich das Absorptionsmaximum um $70 \mathrm{~cm}^{-1} \mathrm{zu}$ höheren Wellenzahlen. Die Verschiebung des Absorptionsmaximums lässt sich mit einer Abnahme der lokalen Dichte am Ort des Chromophors mit steigender Temperatur erklären und ist im Hauptphasenübergang besonders ausgeprägt. 
Auf die nachfolgend vorgestellten Photon-Echo-Experimente sollte diese Verschiebung vernachlässigbare Auswirkungen haben, da die Halbwertsbreite der in den Experimenten verwendeten Pulse ca. $800 \mathrm{~cm}^{-1}$ beträgt.

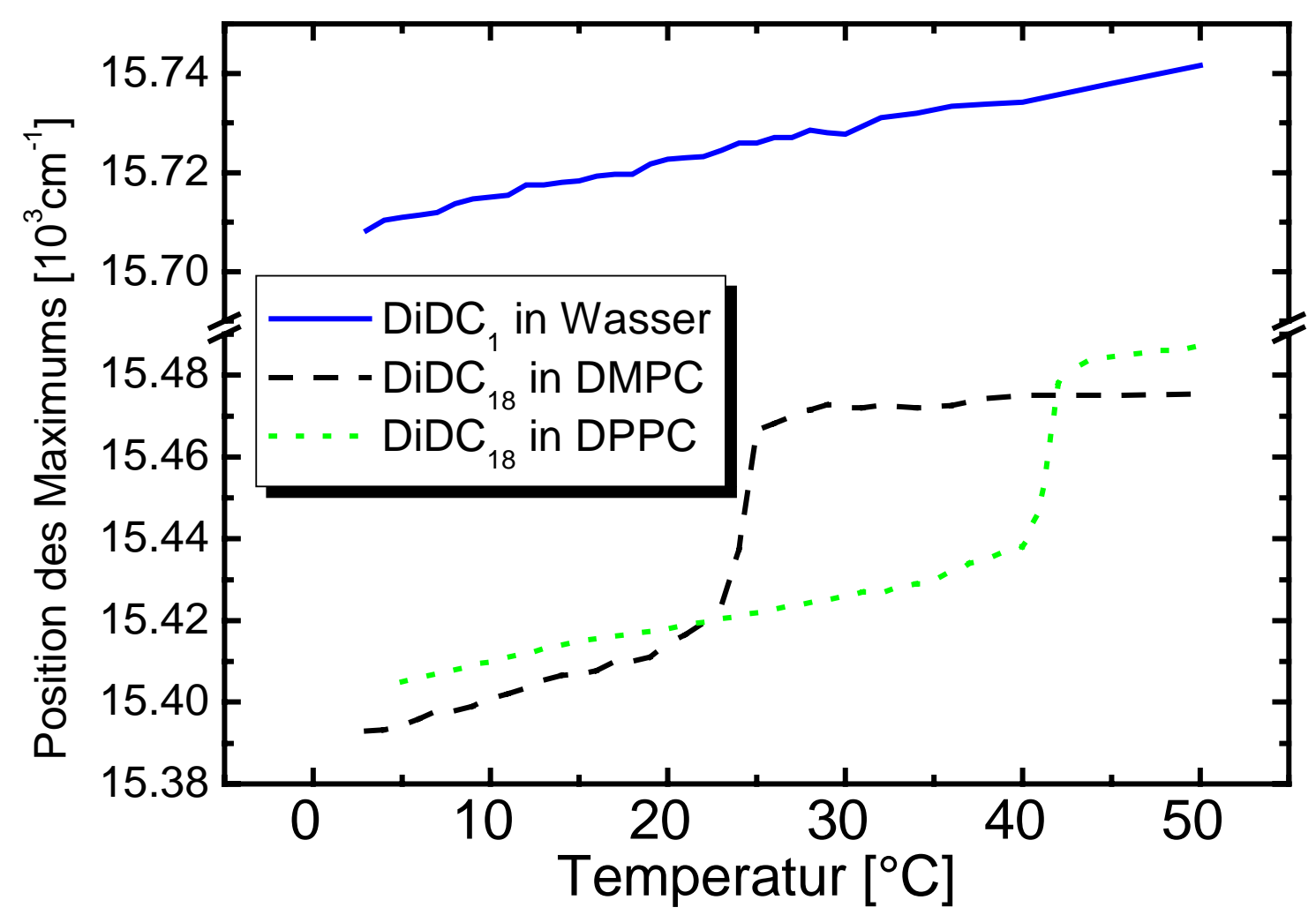

Abbildung 6.7: Position des Maximums der resonanten Absorption von DiDC ${ }_{1}$ im Wasser und DiDC $_{18}$ in der Membran in Abhängigkeit der Temperatur.

\section{d) Bestimmung der Schwingungsstruktur des Chromophors}

Zur Bestimmung der Schwingungsstruktur des verwendeten Chromophors wurden Messungen sowohl in der Zeit- als auch in der Frequenzdomäne durchgeführt.

Ein geeignetes Werkzeug zur Analyse der Schwingungsstruktur in der Zeitdomäne stellt die in Kapitel 4.6 vorgestellte transiente-Gitter-Spektroskopie (TG) dar. Bei dieser Methode werden Schwingungswellenpakete im elektronischen Grundzustand des Chromophors präpariert, die auf die resonante, impulsiv-stimulierte RamanStreuung zurückzuführen sind. Zusätzlich präpariert „impulsive“ Absorption des Anregungslichts Schwingungskohärenzen im elektronisch angeregten Zustand des Chromophors. Solche TG-Messungen sind äquivalent zu frequenzaufgelösten 
Ramanstreuexperimenten, wobei letztere lediglich Schwingungsmoden des Grundzustandes nachweisen können.

Ramanstreuexperimente können resonant oder nicht-resonant durchgeführt werden.

Bei den resonanten Experimenten wird unterschieden, ob der eingestrahlte Laserpuls eine geringere (pre) oder eine höhere Frequenz (post) als das Absorptionsmaximum des Farbstoffs aufweist.

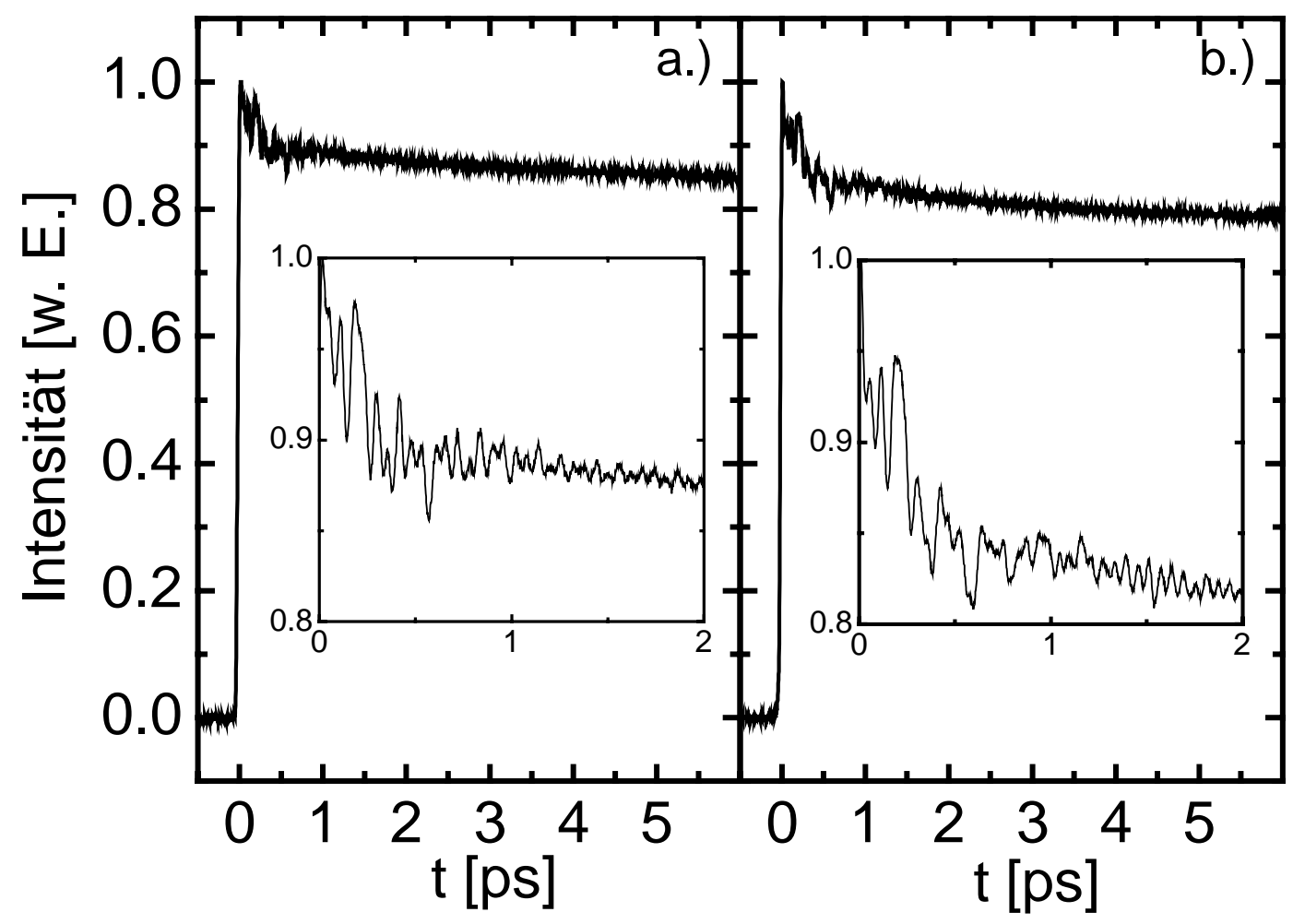

Abbildung 6.8: Messung mit der transienten-Gitter-Spektroskopie an a.) DiDC ${ }_{18}$ in DMPC und b.) DiDC $_{1}$ in Wasser.

In Abbildung 6.8 sind die Ergebnisse der transienten-Gitter-Messungen für $\mathrm{DiDC}_{18}$ in DMPC (a.) und DiDC 1 in Wasser (b.) abgebildet. Die dargestellten Messungen weisen große Gemeinsamkeiten in der Schwingungsstruktur auf. Lediglich das zeitliche Ablinken der Signale innerhalb der ersten 2 ps erfolgt in Wasser deutlich schneller (Einblendung in Abbildung 6.8).

Nicht oszillatorische Anteile zu den in Abbildung 6.8 gezeigten Signalen werden zunächst subtrahiert. Anschließend wird durch Fouriertransformation das Schwingungsspektrum des Chromophors berechnet. Schwingungen mit Frequenzen kleiner als $80 \mathrm{~cm}^{-1}$ wurden aus dem Spektrum entfernt, da sie auf Grund ihrer großen Amplitude höherfrequente Beiträge überdecken würden. 
Bei den Spektren von $\operatorname{DiDC}_{1}$ (Abbildung 6.9 a.) und b.)) ist eine qualitativ gute Übereinstimmung des TG-Spektrums mit dem frequenzaufgelösten Ramanspektrum bis hin zu $1400 \mathrm{~cm}^{-1}$ zu beobachten. Beim Vergleich der Spektren ist zu beachten, dass die Amplituden des fouriertransformierten TG-Signals modifiziert sind, da die spektrale Breite des Laserpulses nicht berücksichtigt wurde. Abweichungen bezüglich der Amplituden resultieren weiterhin aus den unterschiedlichen Resonanzbedingungen beider Experimente.

Die gute Übereinstimmmung zwischen frequenzaufgelöstem Spektrum und TGSpektrum deutet darauf hin, dass die Schwingungsstruktur des Chromophors im angeregten Zustand nahezu identisch mit der des elektronischen Grundzustandes ist. Das Spektrum von $\mathrm{DiDC}_{18}$ in DMPC weist eine große Ähnlichkeit zu dem Spektrum von DiDC 1 in Wasser auf. Die Frequenzen sind lediglich um 5 bis $10 \mathrm{~cm}^{-1}$ zueinander verschoben. Der auffälligste Unterschied besteht jedoch in der Verschmelzung des Tripletts bei $550 \mathrm{~cm}^{-1}$ zu einem Singulett. Die Ursache für diesen Sachverhalt ist unbekannt, da die Schwingungsstruktur des Chromophors bisher nur teilweise zugeordnet wurde [129-131]. Eine mögliche Erklärung wäre die Aggregation des Farbstoffes innerhalb eines Vesikels, wie sie von Katsumata und Mitarbeitern tatsächlich beobachtet werden konnte [132]. Die Bildung von Aggregaten geht jedoch mit einer drastischen Veränderung des Absorptionsspektrums einher (siehe Kapitel 6.1 a.)). Diese Möglichkeit kann in dieser Arbeit jedoch ausgeschlossen werden, da eine Änderung des Absorptionsspektrums hier nicht beobachtet werden konnte.

Interessanterweise stimmen die Spektren von DiDC $_{1}$ in Wasser mit Ramanspektren desselben Chromophors in $\mathrm{KBr}$-Proben quantitativ überein, wenn von einer zusätzlichen Bande knapp unterhalb der niedrigsten Bande des Tripletts abgesehen wird [129].

Aufgrund dieser Aspekte ist es jedoch nicht einsichtig, weshalb das Spektrum von $\mathrm{DiDC}_{18}$ in DMPC ein Singulett anstelle eines Tripletts aufweist. Sato und Mitarbeiter konnten die $576 \mathrm{~cm}^{-1}$-Komponente des Tripletts von DiDC 1 der Deformationsschwingung der Polymethinkette zuordnen, welche die beiden Indolfragmente miteinander verbindet. Die Komponente bei $550 \mathrm{~cm}^{-1}$ und $532 \mathrm{~cm}^{-1}$ resultieren dagegen aus Biegeschwingungen der $\mathrm{C}-\mathrm{C}\left(\mathrm{CH}_{3}\right)_{2}-\mathrm{C}$-Gruppe im Indolfragment [129]. Die drastische Veränderung der Triplettstrukturen durch die 
Verankerung des Chromophors an der Wasser-Lipid-Grenzschicht bleibt allerdings weiterhin unklar, da wie bereits erwähnt eine Aggregatbildung aufgrund der Absorptionsspektren ausgeschlossen werden kann.

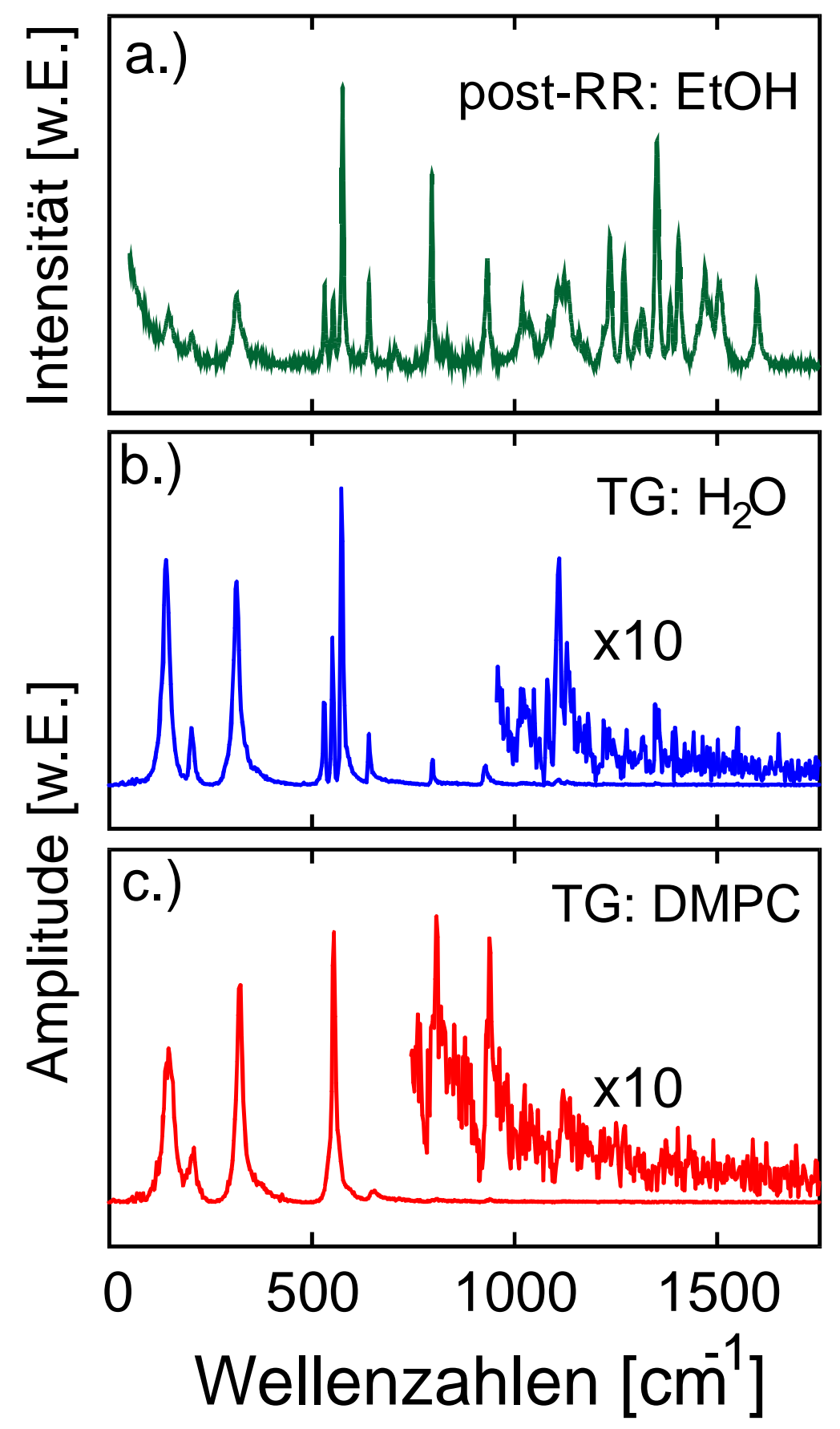

Abbildung 6.9: a.) Post-resonantes Ramanspektrum von DiDC $_{1}$ in Ethanol;

b.) Frequenzamplitudenspektrum der TG-Messung von DiDC $_{1}$ in Wasser;

c.) Frequenzamplitudenspektrum der TG-Messung von DiDC $_{18}$ in DMPC. 


\subsection{Zwei-Puls-Echo-Messungen (2-PE)}

Um einen Überblick zur Solvatationsdynamik in polaren Flüssigkeiten zu erhalten, wurden zunächst Zwei-Puls-Echo-Experimente durchgeführt. Bei einer phänomenologischen Trennung der Zeitskalen in homogene (sehr schnelle) und inhomogene (sehr langsame) Fluktuationen der Umgebung sollte das zeitlich integrierte 2-Puls-Echo-Signal als Funktion der relativen Verzögerung $t_{12}$ der beiden Pulse exponentiell mit der Zeitkonstanten $\mathrm{T}_{2} / 4$ abklingen (siehe Kapitel 4). In diesem sogenannten Bloch-Limit lassen sich die strukturellen Fluktuationen mit einer einzigen Zeitkonstanten beschreiben. Die Fluoreszenzlebensdauer kann beim betrachteten System vernachlässigt werden, da sie mit ca. $1 \mathrm{~ns}$ sehr viel größer ist als die Zeitskalen auf der das Echo abklingt [133, 134].

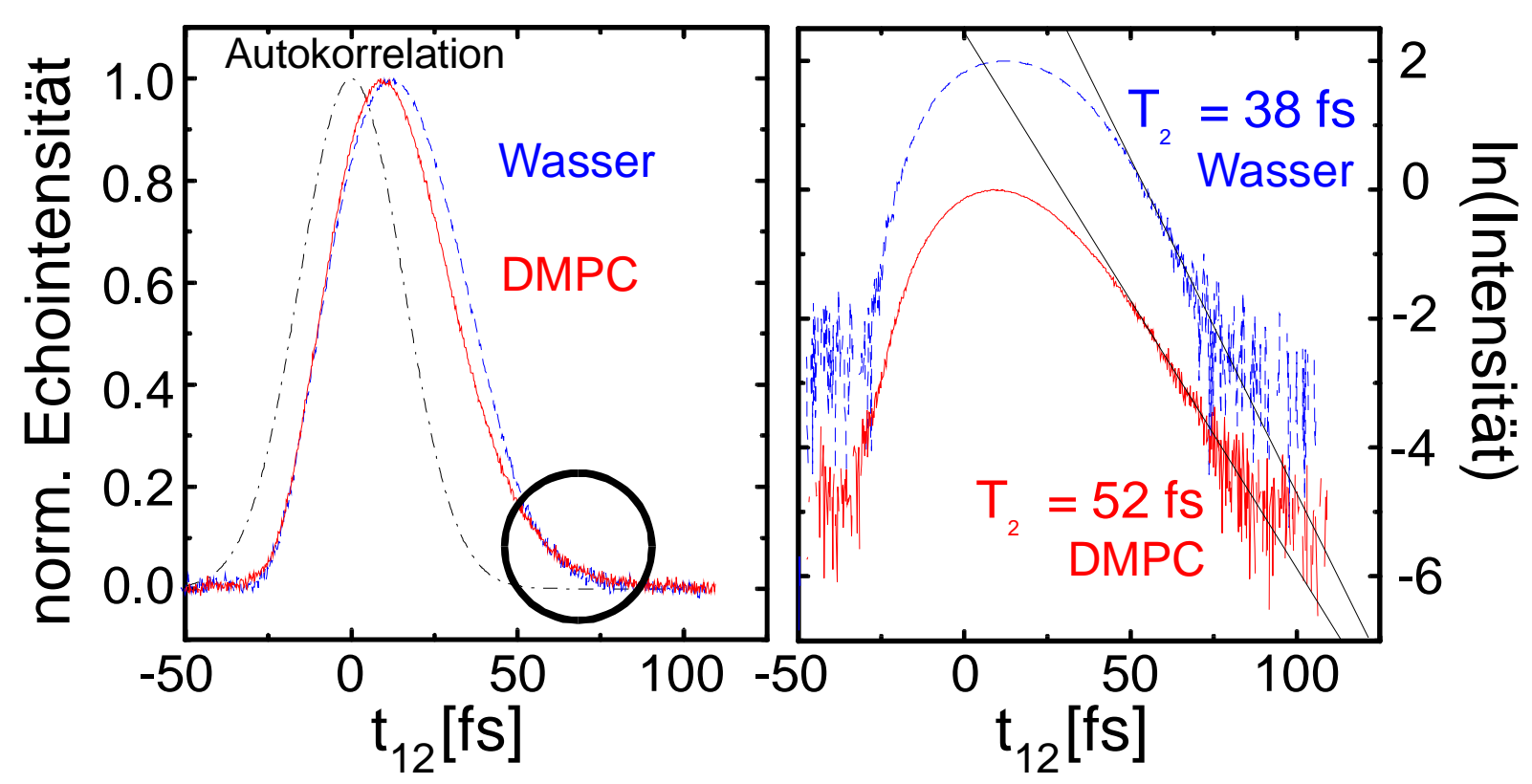

Abbildung 6.10: 2-PE-Signale von DiDC $_{1}$ in Wasser und von DiDC $_{18}$ an der LipidmembranWasser-Grenzschicht aufgetragen gegen die Zeit $t_{12}$ zwischen den beiden eingestrahlten Pulsen.

In Abbildung 6.10 sind die gemessenen 2-PE-Signale von DiDC $_{1}$ in Wasser bzw. $D_{i D C} 18$ in DMPC gegen die Verzögerung $t_{12}$ aufgetragen. Des Weiteren wurde eine Autokorrelationsfunktion zur Festlegung des Nullpunktes hinzugefügt. Die linke Auftragung zeigt die Echointensität in einer linearen, die rechte in einer halblogarithmische Darstellung. Die lineare Auftragung veranschaulicht, dass beide 
Signale stark asymmetrisch und vom Nullpunkt verschoben sind. Eine exponentielle Anpassung (siehe rechts) liefert $\mathrm{T}_{2}$-Zeiten von 52 fs für den Farbstoff $\mathrm{CiDC}_{18}$ in DMPC und 38 fs für DiDC ${ }_{1}$ in Wasser. Da die Anpassung jedoch nur einen kleinen Bereich des Signals (Kreis in Abbildung 6.10) repräsentiert, geben die unterschiedlichen Phasenrelaxationszeiten $T_{2}$ lediglich einen ersten Hinweis auf verschiedene Solvatationsdynamiken in den betrachteten Umgebungen der Farbstoffe.

Dieses nicht-exponentielle Abklingen, sowie die Maximumsverschiebung in Bezug auf den Nullpunkt, kann nur damit erklärt werden, dass sich das System nicht innerhalb des Bloch-Limits beschreiben lässt. Die geforderte Zeitskalentrennung ist somit nicht erfüllt. Dieses Ergebnis entspricht den in der Literatur dokumentierten Arbeiten über Solvatationsdynamik in polaren Flüssigkeiten [135-140].

Aufgrund der in den Zwei-Puls-Echo-Experimenten gewonnenen Ergebnisse, wurden zu einer detaillierten Klärung der Solvatationsdynamik Drei-Puls-Echo-Experimente durchgeführt. 


\subsection{Drei-Puls-Echo-Peakshift-Messungen (3-PEPS) von $\mathrm{DiDC}_{1}$ in Wasser}

Für die Charakterisierung der Solvatationsdynamik in wässrigen Volumenphasen wird zunächst eine 3-PEPS-Messung bei $26^{\circ} \mathrm{C}$ vorgestellt. Anhand dieser Messung wird im folgenden Kapitel eine Zuordnung der verschiedenen Zeitskalen zu bestimmten molekularen Dynamiken von Wasser vorgenommen. Eine anschließende Frequenzanalyse der 3-PEPS-Daten gibt im Vergleich mit den durch Messungen des transienten Gitters erhaltenen Frequenzen einen weiteren Einblick in die Solvatationsprozesse, die Chromophor spezifisch sind.

Die bei $26^{\circ} \mathrm{C}$ gemessene Solvatationsdynamik wird im zweiten Teil des Kapitels durch 3-PEPS-Messungen bei weiteren Temperaturen ergänzt. Die zugeordneten Solvatationsmoden werden durch diese Messungen auf ihre Temperaturabhängigkeit untersucht.

\subsubsection{Analyse der Solvatationskorrelationsfunktion des Wassers bei Zimmer- temperatur}

In Abbildung 6.11 sind die aus den 3-Puls-Echo-Experimenten gewonnenen Peakshifts in Abhängigkeit von der Verzögerungszeit $\mathrm{t}_{23}$ aufgetragen. Die Auftragung mit der logarithmierten Abszisse (Abbildung 6.11 a.)) stellt den gesamten Messbereich von $t_{23}=2$ fs bis $t_{23}=300$ ps dar. Der Graph in Abbildung 6.11 b.) konzentriert sich demgegenüber auf den oszillatorischen Anteil des 3-Puls-EchoPeakshifts im Bereich von $t_{23}=0$ bis 3 ps. Die Zeitintervalle zwischen den einzelnen Messpunkten $t_{23}$ betragen 2 fs für den Kurzzeitbereich ( $t_{23}=0-30 \mathrm{fs}$ ) und $10 \mathrm{fs}$ für den oszillatorischen Abschnitt $\left(\mathrm{t}_{23}=0.03-3 \mathrm{ps}\right)$. Im Bereich von $\mathrm{t}_{23}=3-300 \mathrm{ps}$ werden 100 Messpunkte mit exponentiell anwachsender Verzögerung bestimmt. Es wurden drei individuelle 3-PEPS-Kurven in Boxcargeometrie gemittelt. Der Verlauf der Peakshifts kann mit drei Exponentialfunktionen und einer Gauß-Funktion gemäß Gleichung 6.3 angepasst werden:

$$
I\left(t_{23}\right)=\sum_{i=1}^{n} A_{i} \exp \left(-\frac{t_{23}}{\tau_{i}}\right)+A_{n+1} \exp \left(-\frac{t_{23}^{2}}{\sigma^{2}}\right) .
$$

\section{Gleichung 6.3}

Die erhaltenen Parameter sind in Tabelle 6.1 zusammengefasst. 

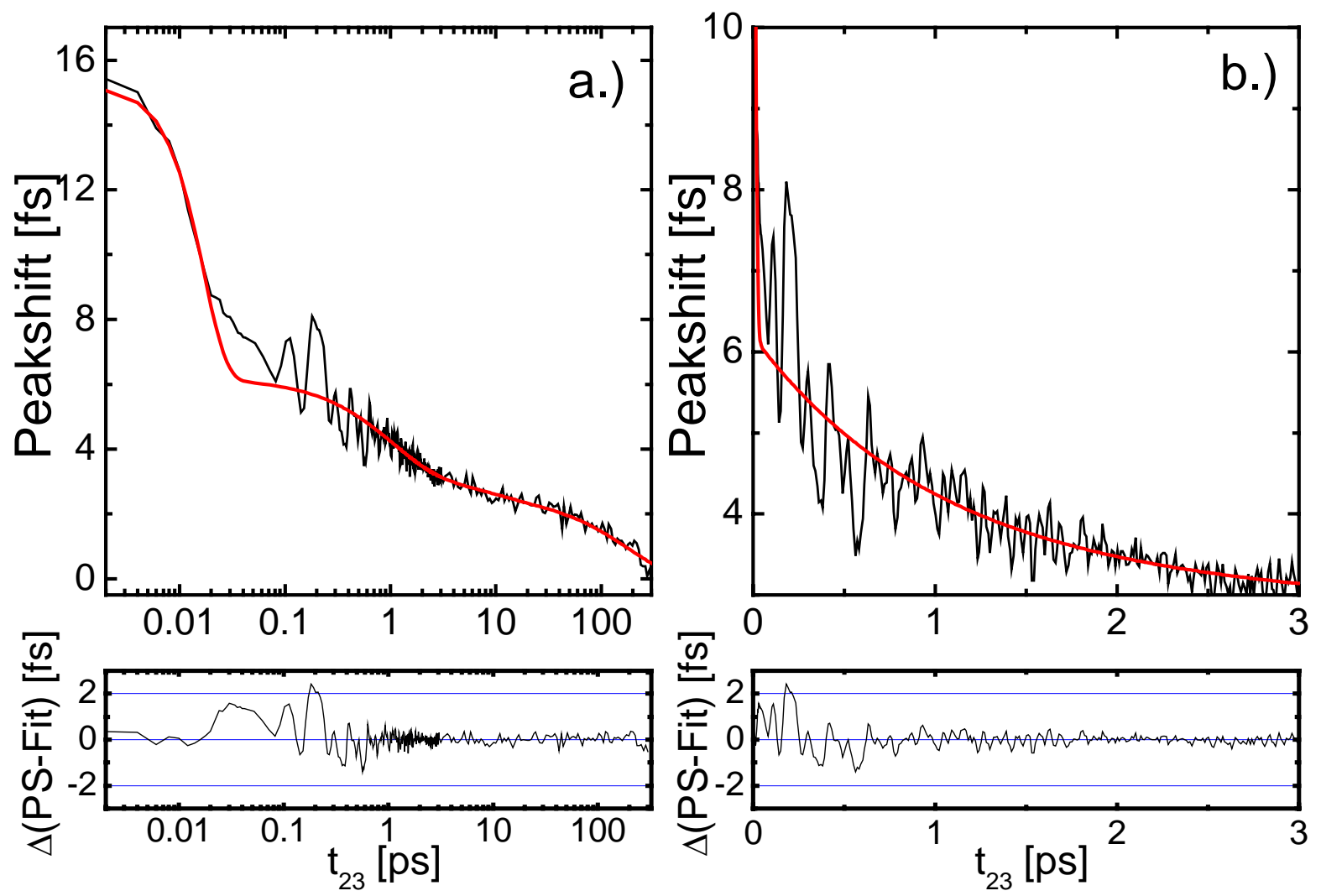

Abbildung 6.11: 3-PEPS-Kurve von $\mathrm{DiDC}_{1}$ in Wasser bei $26^{\circ} \mathrm{C}$ mit einer Anpassung aus der Summe dreier Exponentialfunktionen und einer Gaußfunktion mit den Zeitkonstanten $17 \mathrm{fs}, 1 \mathrm{ps}, 7.5 \mathrm{ps}$ und $180 \mathrm{ps}$. Unterhalb den 3-PEPS-Kurven sind jeweils die Residuen, d.h. die Differenz aus Messdaten und Anpassung, abgebildet.

\begin{tabular}{|c|c|c|c|c|}
\hline $\mathbf{i}$ & $\mathbf{A}_{\mathbf{i}}$ & $\tau_{\mathbf{i}}$ & $\sigma$ & $\mathbf{A}_{\mathbf{i}} / \Sigma \mathbf{A}_{\mathbf{i}}$ \\
\hline 1 & $2.5 \mathrm{fs}$ & $180 \mathrm{ps}$ & - & $16.7 \%$ \\
\hline 2 & $0.7 \mathrm{fs}$ & $7.5 \mathrm{ps}$ & - & $4.7 \%$ \\
\hline 3 & $2.8 \mathrm{fs}$ & $1 \mathrm{ps}$ & - & $18.7 \%$ \\
\hline 4 & $9 \mathrm{fs}$ & - & $17 \mathrm{fs}$ & $60.0 \%$ \\
\hline
\end{tabular}

Tabelle 6.1: Zusammenstellung der ermittelten Parameter gemäß Gleichung 6.3 für DiDC $_{1}$ in Wasser bei $26^{\circ} \mathrm{C}$. 
Abbildung 6.11 zeigt außerdem die Residuen, die sich aus der Differenz der Messdaten und der Anpassungsfunktion ergeben. Sie repräsentieren im Wesentlichen die oszillatorischen Anteile der 3-PEPS-Signale. Im Folgenden wird versucht, die einzelnen Zeitkonstanten in einen Zusammenhang mit der Solvatationsdynamik des Wassers zu bringen.

In früherer Arbeit von Vöhringer und Mitarbeitern konnte das Abklingen der 3-PEPS nur bis zu einer maximalen Verzögerung $t_{23}$ von 50 ps gemessen werden [141]. Die vorliegenden Messungen mit einem auf 300 ps ausgedehnten Messbereich liefern eine langsamste Komponente mit einer Relaxationszeit $\tau_{1}=180$ ps und zeigen damit, dass die als Inhomogenität bezeichneten Beiträge immer noch auf langen Zeitskalen abklingen. Die ermittelte Zeitkonstante hat allerdings nur eine Genauigkeit von \pm 100 ps. Der Grund für diese Ungenauigkeit liegt bei dem noch immer für diese Zeitkonstante zu kurzem Messbereich. Bei Messungen in der Boxcargeometrie kommt erschwerend hinzu, dass der Peakshift nur relativ zur Autokorrelation bestimmt wird (siehe Kapitel 5.3). Aufgrund dieser Art der Peakshiftbestimmung kann es durch Gruppenlaufzeitunterschiede der Pulse zu einer Fehlbestimmung des Zeitnullpunktes kommen. Dieser Fehler wirkt sich auf die Genauigkeit der Zeitkonstanten der langsamsten Komponente des 3-PEPS negativ aus. Es bleibt allerdings festzuhalten, dass der Peakshift nach 300 ps noch nicht abgeklungen ist. Der endliche Peakshift bei den größten Verzögerungen $t_{23}$ reflektiert das Einsetzen langsamer spektraler Diffusionsvorgänge in der Flüssigkeit. Dies belegen insbesondere die weiter unten abgebildeten temperaturabhängigen 3-PEPSKurven (Abbildung 6.15), welche in der Dreiecksgeometrie gemessen wurden und daher den absoluten Peakshift wiedergeben.

Das langsame Abklingen des 3-PEPS mit einer Zeitkonstanten $\tau_{1}=180$ ist vermutlich auf die kollektive Rotationsdiffusion des Lösungsmittels zurückzuführen. Auf ähnlichen Zeitskalen findet allerdings auch die Rotationsdiffusion des Farbstoffs statt [142, 143]. Eine genaue Zuordnung ist auf Grund der oben erwähnten Ungenauigkeiten und der unvollständigen Informationen bezüglich dieser kollektiven Rotationsmoden des Wassers zur Zeit nicht möglich.

Das exponentielle Abklingen der 3-PEPS-Funktion im Bereich zwischen 3 und 50 ps wird mit einer Zeitkonstante von $\tau_{2}=7.5$ ps beschrieben. Aufgrund der geringen 
Amplitude (siehe Tabelle 6.1) besitzt die Zeitkonstante dieser Komponente nur eine Genauigkeit von 1 ps. Trotz der schwierigen Bestimmung dieser Zeitkonstanten, konnte sie in wiederholten Messungen mit der angegeben Genauigkeit reproduziert werden.

Diese Abklingzeit ist in guter Übereinstimmung mit der Reorientierungskorrelationszeit einzelner Wassermoleküle, wie sie aus Messungen der dielektrischen Relaxation (DR) [144] und longitudinalen NMR-Relaxationszeiten [145-147] sowie aus zeitaufgelösten THz-Experimenten [148, 149] bestimmt wurde. Neueste Messungen des Raman-induzierten optischen Kerr-Effekts (OKE) [150] an Wasser zeigten, dass die Zeitkonstante der Rotationsdiffusion, die aus NMR-Experimenten [151] über die Spin-Gitter-Relaxation $R_{1}$ erhalten wird, und die langsamste Zeitkonstante aus diesen OKE-Experimenten die gleiche Moleküldynamik beschreiben [150].

Die Relaxation im Bereich von 50 fs bis 3 ps kann mit einer Zeitkonstanten von $\tau_{3}=1$ ps angepasst werden. Sie lässt sich mit einer Genauigkeit von \pm 0.1 ps bestimmen. Lediglich die Oszillationen, die weiter unten diskutiert werden, erschweren eine Ermittlung dieser Zeitkonstanten.

Ein Vergleich mit den bereits erwähnten OKE-Daten [150] und Daten aus depolarisierten Ramanexperimenten [152] zeigt, dass diese Komponente mit der Relaxation einer übergedämpften, kollektiven Translationsmode des reinen Wasser übereinstimmt. Des Weiteren ist dieses Zeitfenster von kritisch gedämpften, gehinderten Translationsmoden des reinen Lösungsmittels bestimmt. Diese Moden beschreiben die Streckung und Biegung von Wasserstoffbrückenbindungen in Wasserclustern unterschiedlicher Größe. Nach Arbeiten von Walrafen und Mitarbeitern [153] können diese Bewegungen mit den transversalen akustischen (TA) und longitudinalen akustischen (LA) Phononen von amorphem und kristallinem Eis in Verbindung gebracht werden (TA-Bande bei $60-70 \mathrm{~cm}^{-1}$, LA-Bande bei $\left.164-181 \mathrm{~cm}^{-1}\right)$.

Schließlich soll das Abklingen des 3-PEPS-Funktion im Bereich unter $50 \mathrm{fs}$, das durch eine Gaußfunktion mit der Zeitkonstanten von $\sigma_{4}=17$ fs angepasst werden kann, diskutiert werden. Da das Abklingen der 3-PEPS-Funktion um $t_{23}=0$ auf die Form der System-Bad-Korrelationsfunktion unempfindlich ist, könnte der 
Anfangsbereich ebenso mit einer Exponentialfunktion angepasst werden [136, 154, 155]. Eine Gauß-Funktion entspricht jedoch dem theoretisch erwartetem Verlauf der Solvatationskorrelationsfunktion [76].

Ursache dieser ultraschnellen Dynamik könnten nach Jimenez und Mitarbeitern [156] die Librationsmoden der Wassermoleküle in der ersten Hydratationsschale des Chromophors sein. Gegen diese Interpretation spricht jedoch die Tatsache, dass die schnellsten intermolekularen Moden des Wassers eine Schwingungsperiode von ungefähr 67 fs aufweisen. In Infrarot- und Ramanspektren werden diese Moden um $500 \mathrm{~cm}^{-1}$ sichtbar.

Bei 3-PEPS-Experimenten wird die Zuordnung dieser ultrakurzen Zeitkonstanten erschwert, da die 3-PEPS-Funktion $S\left(t_{23}\right)$ nur für den langsamen Teil von $M\left(t_{23}\right)$ in einer direkten Beziehung zur Solvatation steht (Kapitel 4). Der ultraschnelle Anteil von $\mathrm{S}\left(\mathrm{t}_{23}\right)$ mit einer Zeitkonstanten, die kürzer als die Pulshalbwertsbreite ist, kann nicht mehr zum langsamen Teil $\mathrm{M}_{\text {slow }}\left(\mathrm{t}_{23}\right)$ gezählt werden. Die 3-PEPS-Funktion $\mathrm{S}\left(\mathrm{t}_{23}\right)$ überschätzt vielmehr die Amplitude des schnellen Anteils der Solvatationsfunktion $M_{\text {fast }}\left(t_{23}\right)$ und unterschätzt seine Zeitkonstante. Eine Solvatationskorrelationsfunktion, die aus einer theoretisch hergeleiteten spektralen Dichte berechnet wurde, zeigt dementsprechend eine relative Amplitude von $58 \%$ und eine Zeitkonstante von 28 fs [154]. Der Grund dieser ultrakurzen Komponente der Solvatation bleibt trotz vielseitiger Interpretationsversuche unklar. Mehrere Arbeitsgruppen haben versucht, mit Hilfe molekulardynamischer Simulationen [157, 158] und dielektrischen Kontinuumstheorien [159] ihren Ursprung zu erörtern. Die so erhaltenen Zeitkonstanten liegen allerdings bei weniger als 25 fs mit einer Amplitude von 7090\%. Diese Parameter sind nicht mit den von Huppert und Mitarbeitern [160] sowie Lang und Mitarbeitern [154] ermittelten Werten konsistent. Aus diesen Gründen wird im Folgenden von einer weiteren Betrachtung der ultrakurzen Komponente Abstand genommen.

Neben dem exponentiellen Abklingverhalten fallen im Bereich von 0 bis 10 ps Oszillationen der 3-PEPS-Kurve auf (Abbildung 6.11). Diese Schwingungen resultieren aus intramolekularer Wellenpaketdynamik des Farbstoffes [75]. Nach Subtraktion der Gauß- und Exponentialfunktionen wird deshalb an den Residuen $\left(S\left(t_{23}\right)-I\left(t_{23}\right)\right)$ eine Fourieranalyse durchgeführt.

Das so gewonnene Amplitudenpsektrum der 3-PEPS-Daten im Vergleich zum TGSpektrum aus Abschnitt 6.1 ist in Abbildung 6.12 aufgetragen. Zwischen den beiden 
Spektren besteht eine sehr gute Übereinstimmung. Lediglich die Amplituden der Banden sind verschieden. Auch die im Spektrum des transienten Gitters deutlich sichtbare Bande bei etwa $800 \mathrm{~cm}^{-1}$ ist im 3-PEPS-Spektrum erkennbar. Eine Diskussion der Schingungsstruktur des Chromophors kann Kapitel 6.1 d.) entnommen werden.

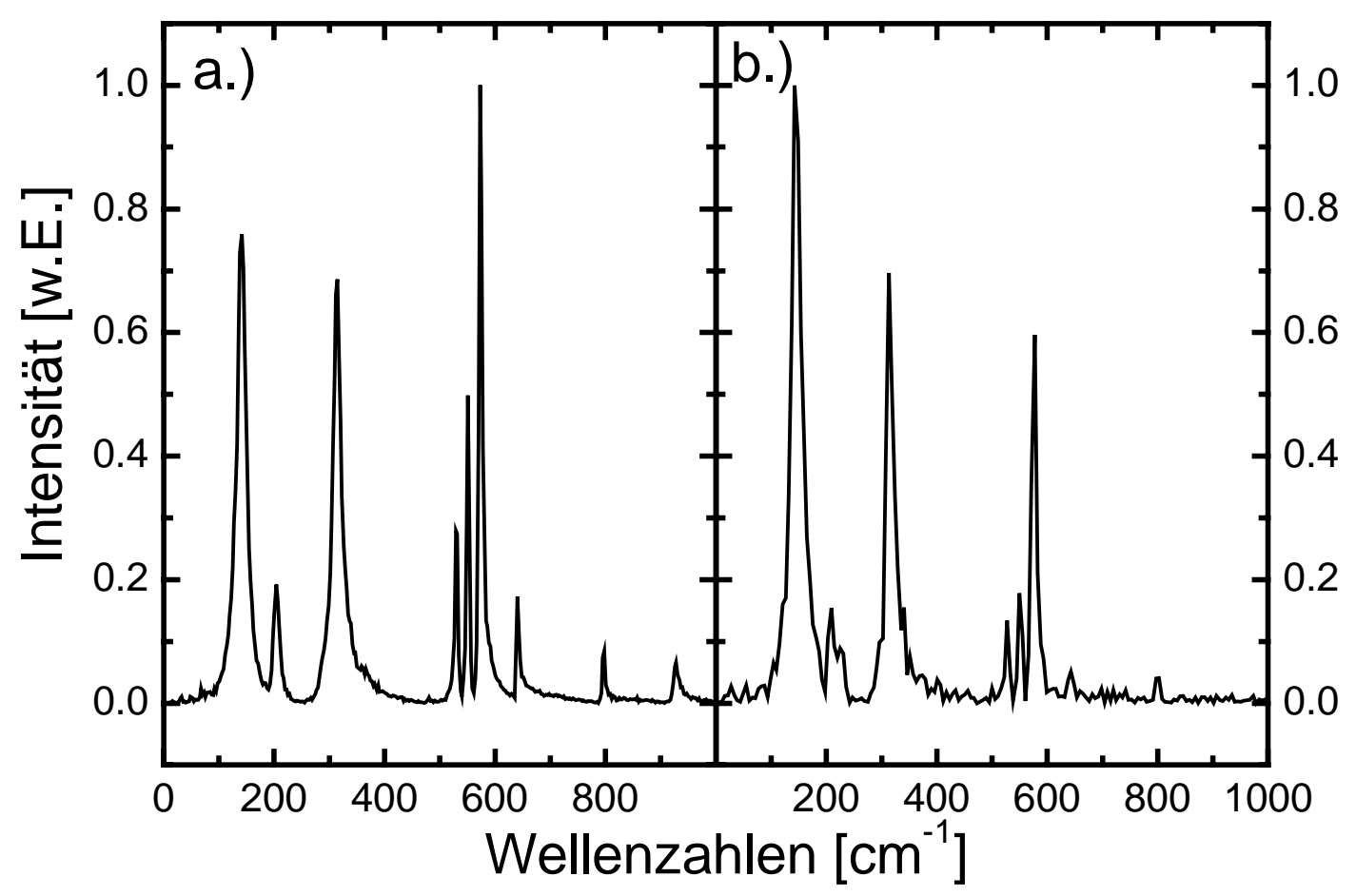

Abbildung 6.12: Fourierspektrum von $\mathrm{DiDC}_{1}$ in Wasser bei $26^{\circ} \mathrm{C}$; Die Amplituden $\mathrm{B}_{\mathrm{i}} \mathrm{zu}$ Schwingungen mit $\omega_{i}<80 \mathrm{~cm}^{-1}$ sind in beiden Graphen Null gesetzt, um einen besseren Vergleich der Spektren zu ermöglichen:

a.) TG-Messung; b.) 3-PEPS-Messung.

Zur Konsistenzprüfung wurden die experimentellen Daten einer LP-SVD Analyse (,Iinear prediction-singular value decomposition“) unterworfen. Der Simulationsalgorithmus zerlegt die Daten in eine Überlagerung exponentiell gedämpfter Schwingungen gemäß [161, 162]:

$$
F(t)=\sum_{i} B_{i} \cos \left(\omega_{i} \cdot t+\phi_{i}\right) \cdot \exp \left(-\frac{t}{\tau_{i}}\right)
$$

\section{Gleichung 6.4}


wobei $B_{i}$ die Amplitude, $\omega_{i}$ die Frequenzen, $\phi_{i}$ die relativen Phasen und $\tau_{i}$ die Dämpfungskonstanten der individuellen Komponenten darstellen.

Die Summe aus oszillatorischen (Gleichung 6.4) und nicht-oszillatorischen (Gleichung 6.3) Komponenten, die aus der Analyse gewonnen wurden, ist zusammen mit den experimentellen Daten in Abbildung 6.13 dargestellt. Die Residuen unterhalb der Graphen stellen die Qualität der Anpassung heraus. Das Rauschen $\triangle \mathrm{PS}$ beträgt weniger als $\pm 0.4 \mathrm{fs}$, wenn von einer geringen Modulation im Ultrakurzzeitbereich bei $t_{23}=10$ fs abgesehen wird. Dieses Rauschen entspricht der Messgenauigkeit der vorgestellten Peakshift-Messung.
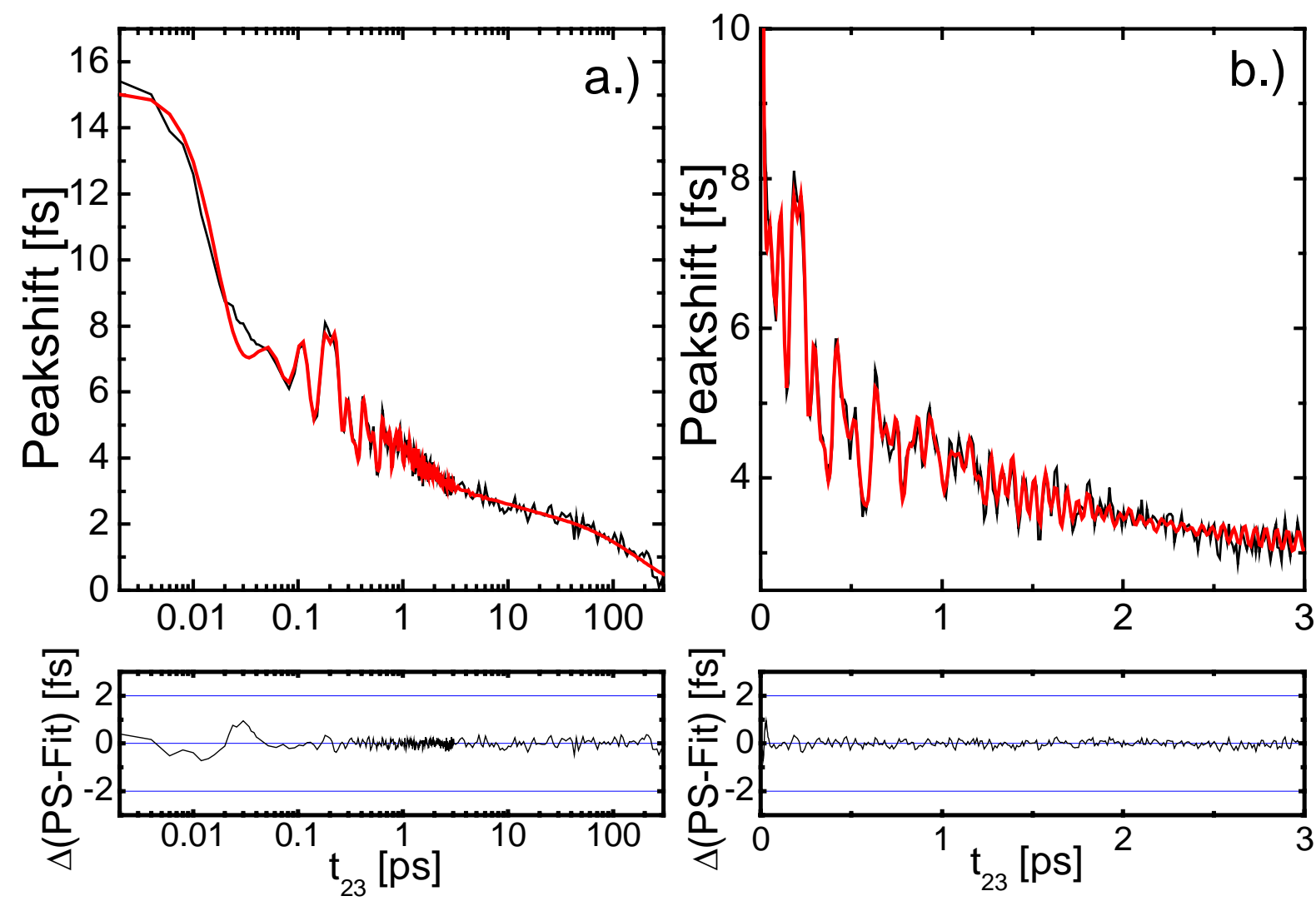

Abbildung 6.13: 3-PEPS-Messkurven von $\mathrm{DiDC}_{1}$ in reinem Wasser bei $26^{\circ} \mathrm{C}$ inklusive einer Anpassung aus oszillatorischen und nicht-oszillatorischen Anteilen. Im unteren Teil sind die jeweiligen Residuen abgebildet.

Die spezifischen Oszillationen des Farbstoffes wurden bei der Diskussion der Messungen des transienten Gitters erörtert und sowohl die langsamste als auch die ultraschnelle Zeitkonstante bedürfen genauerer Untersuchungen. Für einen Vergleich mit anderen Arbeiten verbleiben noch die Relaxationszeiten von $\tau_{3}=1 \mathrm{ps}$ und $\tau_{2}=7.5 \mathrm{ps}$. 
Erste Messungen zur Bestimmung der ultraschnellen Solvatationsdynamik in Wasser wurden von Barbara und Mitarbeitern [163] durchgeführt. In diesen Arbeiten wurden zeitaufgelöste Messungen zur Bestimmung der dynamischen Stokes-Verschiebung eines Coumarin-Farbstoffes mittels Fluoreszenzkonvertierungsspektroskopie durchgeführt. Die dynamische Stokes-Verschiebung wurde mit zwei Exponentialfunktionen mit Abklingzeiten von 0.16 ps und 1.2 ps angepasst. Ihr amplitudengewichteter Mittelwert entspricht einer Zeitkonstanten von $\tau=0.86$ ps. Die Gerätefunktion der Messungen entsprach einer Gauß-Funktion mit einer Halbwertsbreite von $280 \mathrm{fs}$. In jüngeren Messungen von Jimenez und Mitarbeitern [156] konnte die Zeitauflösung mit dieser Technik auf 100 fs verbessert werden. Die von innen bestimmte Solvatationskorrelationsfunktion klingt triexponentiell mit zwei schnelleren und einer langsameren Zeitkonstanten von $\tau=0.88$ ps ab.

In einer weiteren Arbeit berechnen Marcus und Mitarbeiter [164] die Solvatationskorrelationsfunktion aus lösungsmittelspezifischen, dielektrischen Dispersionsdaten $\varepsilon(\omega)$. Die innerhalb des dielektrischen Kontinuum-Modells berechnete Solvatationskorrelationsfunktion gibt den Verlauf der oben erwähnten dynamischen Stokes-Verschiebung gut wieder. In diesem Modell wird das Lösungsmittel als uniformes, dielektrisches Kontinuum verstanden, welches lediglich durch die frequenzabhängige dielektrische Funktion $\varepsilon(\omega)$ charakterisiert ist [166, 167, 184, 186, 191-197]. Für den jeweiligen Chromophor wurde eine sphärische oder ellipsoide Form angenommen. Zur Berechnung einer Solvatationskorrelationsfunktion wurde von einer instantanen Präparation des Dipols ausgegangen. Die Berechnungen ergaben eine Zeitkonstante von $\tau=810 \mathrm{fs}$, die sowohl mit der ermittelten Relaxationszeit von $\tau=0.86$ ps aus Fluoreszenzkonvertierungsexperimenten übereinstimmt, als auch mit der Zeitkonstanten der hier vorgestellten 3-PEPS-Experimente von $\tau_{3}=1 \mathrm{ps}$. Die wichtigsten Ergebnisse diese theoretischen Modells werden im Folgenden näher erläutert [164]:

Zunächst wird das Spektrum der dielektrischen Funktion $\varepsilon(\omega)$ in einen hochfrequenten und einen niederfrequenten Bereich unterteilt. Der niederfrequente Anteil kann mit der Debye-Formel im Intervall von $0 \mathrm{~cm}^{-1} \leq \omega \leq 1.6 \mathrm{~cm}^{-1}$ beschrieben werden: 


$$
\varepsilon^{\prime \prime}(\omega)=\left(\varepsilon_{\mathrm{s}}-\varepsilon_{\infty}\right) \frac{\omega \cdot \tau_{\mathrm{D}}}{1+\omega^{2} \cdot \tau_{\mathrm{D}}^{2}}=\left(\varepsilon_{\mathrm{s}}-\varepsilon_{\infty}\right) \frac{\Lambda_{\mathrm{D}} \cdot \omega}{\Lambda_{\mathrm{D}}^{2}+\omega^{2}}
$$

\section{Gleichung 6.5}

Die inverse Debye-Relaxationszeit wird mit $\Lambda_{D}=\tau_{D}{ }^{-1}$ bezeichnet. Die Grenzwerte für dielektrischen Funktion sind die statistische Dielektrizitätskonstante $\varepsilon_{\mathrm{s}}$ für $\omega=0$ und $\varepsilon_{\infty}$ für $\omega \rightarrow \infty$. Der Bereich des Spektrums, in dem eine Näherung mit Hilfe der Debye-Formel zulässig ist, beschreibt die Solvatationskorrelationsfunktion für lange Zeiten. Dieser Zeitbereich ist durch Diffusionsprozesse charakterisiert. Der hochfrequente Bereich hat dagegen Einfluss auf die Solvatationsfunktion zu frühen Zeiten. Diese hohen Frequenzen resultieren aus inter- und intramolekularen Moden des Lösungsmittels.

Marcus und Mitarbeiter stellen des Weiteren klar heraus, dass eine DebyeRelaxation experimentell nur auf Zeitskalen länger als 3 ps beobachtet werden kann. Die berechnete Zeitkonstante von $\tau=810$ fs wird daher zurecht einer Mischung aus Debye-Relaxation und einer Relaxation auf Grund höherfrequenter Moden der Flüssigkeit zugeordnet.

In Übereinstimmung mit den Betrachtungen von Marcus und Mitarbeitern wird die in den 3-PEPS-Experimenten beobachtete Relaxation mit der Zeitkonstanten $\tau_{3}=1$ ps den höherfrequenten Moden des Wassers zugeordnet. Zu diesen Moden gehören die intermolekularen gehinderten Translationsmoden des Wassers.

Die ermittelte Zeitkonstante von $\tau_{2}=7.5$ ps stimmt weiterhin mit der Aussage von Marcus und Mitarbeitern darin überein, dass eine Debye-typische Relaxation auf längeren Zeitskalen als 3 ps zu erwarten ist. Die Zuordnung dieser Zeitkonstante zur diffusiven Reorientierungskorrelationszeit von individuellen Wassermolekülen ist aus diesem Grund mit den theoretischen Ergebnissen zur Debye-Relaxation von Marcus und Mitarbeiten konsistent. Die Übereinstimmung beider Zeitkonstanten mit den Ergebnissen aus Messungen des optisch Kerr-Effektes [150] unterstützen diese Zuordnung. 
Von Fleming und Mitarbeitern [154] wurden ebenfalls 3-PEPS-Messungen veröffentlicht, die die Solvatation von Eosin Y, einem Fluorescein-Derivat, in Wasser beschreiben. In deren Arbeit wurde die Solvatationskorrelationsfunktion ebenfalls mit drei Exponentialfunktionen mit den Zeitkonstanten $17 \mathrm{fs}, 400 \mathrm{fs}$, und $2.7 \mathrm{ps}$ beschrieben. Diese Anpassung wird in Abbildung 6.14 mit den obigen 3-PEPS-Daten dieser Arbeit verglichen. Wie deutlich zu sehen ist, liegt bis zu einer Zeit von 1 ps eine grobe Übereinstimmung vor. Die schnellste Komponente von Fleming und Mitarbeitern ( $\tau=17 \mathrm{fs}$ ) taucht auch in der hier vorgestellten Messung auf. Im Bereich oberhalb von 2 ps divergieren die beiden Datensätze. Die 3-PEPS-Messung von Fleming und Mitarbeitern ist nach 15 ps bereits vollständig auf Null abgefallen, während die in dieser Arbeit vorgestellten Daten selbst nach einigen 100 ps noch nicht abgeklungen ist. Offensichtlich trägt die Komponente der Rotationsdiffusion $\left(\tau_{2}=7.5 \mathrm{ps}\right)$ nicht zur Solvatationsdynamik von Eosin $\mathrm{Y}$ in Wasser bei.

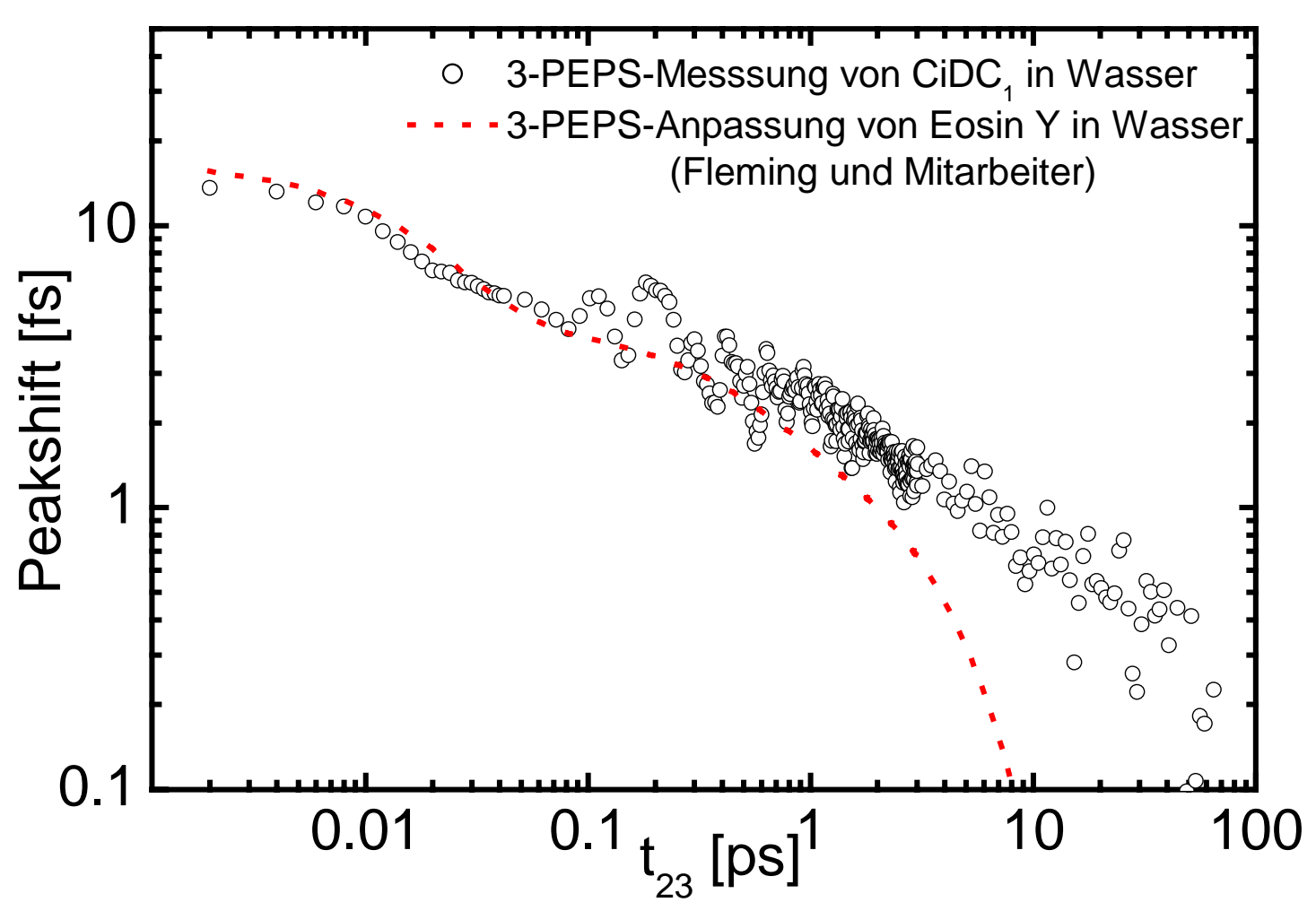

Abbildung 6.14: Auftragung der 3-PEPS-Daten von DiDC $_{1}$ in reinem Wasser sowie der Anpassung der Solvatationsdynamik der Eosin $Y$ Messung in Wasser nach Experimenten von Fleming und Mitarbeitern [154]. 
Fleming und Mitarbeiter versuchten eine quantitative Analyse ihrer Daten vorzunehmen, indem zunächst auf Basis quantenchemischer Rechnungen die Ladungsdichtenverteilungen des Chromophors im Grund- und angeregten Zustand ermittelt wurden. Des Weiteren wurden aus TG-Messungen die Schwingungsfrequenzen des Chromophors abgeschätzt. Ähnlich wie bei Marcus und Mitarbeitern wurde anschließend die Solvatationskorrelationsfunktion mit Hilfe einer dielektrischen Kontinuumstheorie berechnet. Die hierzu notwendige spektrale Dichte der Flüssigkeit wurde aus THz-IR-Daten ermittelt. Die simulierte 3-PEPS-Funktion konnte jedoch ohne zusätzliche Korrekturen die Messdaten innerhalb der Messgenauigkeit nicht wiedergeben. Um eine bessere Übereinstimmung zu erlangen, war es nötig, einen zusätzlichen übergedämpften Brownschen Oszillator mit einer Korrelationszeit von $\tau=8$ ps zur spektralen Dichte künstlich hinzuzufügen. Die spektrale Dichte eines solchen übergedämpften Brownschen Oszillators wird durch

$$
C(\omega)=\frac{2 \cdot \lambda}{\pi} \cdot \frac{\Lambda \cdot \omega}{\Lambda^{2}+\omega^{2}}
$$

\section{Gleichung 6.6}

beschrieben. Die Solvatationskorrelationsfunktion $M(t)$ dieses Brownschen Oszillators klingt exponentiell mit $\Lambda$ ab (siehe Kapitel 4.4).

Im Debye-Bereich sind daher die von Fleming und Mitarbeitern hinzugefügte spektrale Dichte (Gleichung 6.6) und die dielektrische Antwort des Wassers identisch (Gleichung 6.5), da beide eine Zeitkonstante von etwa 8 ps besitzen. Diese Komponente beschreibt die Rotationsdiffusion individueller Moleküle in reinem Wasser. Es bleibt weiterhin unklar, wie dieses Modell, das einen übergedämpften Brownschen Oszillator mit einer Zeitkonstanten von $\tau=8$ ps beinhaltet, die experimentellen 3-PEPS-Daten von Eosin $\mathrm{Y}$ wiedergeben kann, während sich dieselben experimentellen Daten adäquat durch eine Summe von Exponentialfunktionen beschreiben lassen, deren langsamste Zeitkonstante nur 2.7 ps beträgt.

Die Diskrepanz der Daten von Lang und Mitarbeitern zu den hier vorgestellten 3-PEPS-Resultaten beruht möglicherweise auf der Verwendung unterschiedlicher Farbstoffe. Während die gemessene Solvatationsdynamik mit DiDC $_{1}$ als SensorFarbstoff die Rotationsdiffusion individueller Moleküle beinhaltet, scheint das doppelt 
geladene Eosin $Y$ zur Studie von reinem Wasser keine gute Wahl zu sein. Eosin $Y$ stört vielmehr durch die ladungsbedingten Wechselwirkungen mit dem umgebenden Wasser die wassereigene Dynamik. Untersuchungen zur Rotationsdiffusion von Eosin $Y$ deuten an, dass in wässriger Lösung Wasserstoffbrücken zwischen Lösungmittel und Farbstoff ausgebildet werden, die diffusive Bewegungen deutlich behindert [171]. Derartige Effekte sind bei $\mathrm{DiDC}_{1}$ nicht beobachtet worden.

\subsubsection{Temperaturabhängigkeit der Solvatation von DiDC $_{1}$ in Wasser}

Temperaturabhängige 3-PEPS-Messungen in der Boxcargeometrie wurden durchgeführt, um die Zuordnung der obigen Zeitkonstanten durch ihr Temperaturabhängigkeit zu verifizieren. In Abbildung 6.15 ist die 3-PEPS-Funktion gegen die Verzögerungszeit $t_{23}$ für verschiedene Temperaturen $\left(10^{\circ} \mathrm{C}, 25^{\circ} \mathrm{C}, 50^{\circ} \mathrm{C}, 75^{\circ} \mathrm{C}\right)$ aufgetragen.

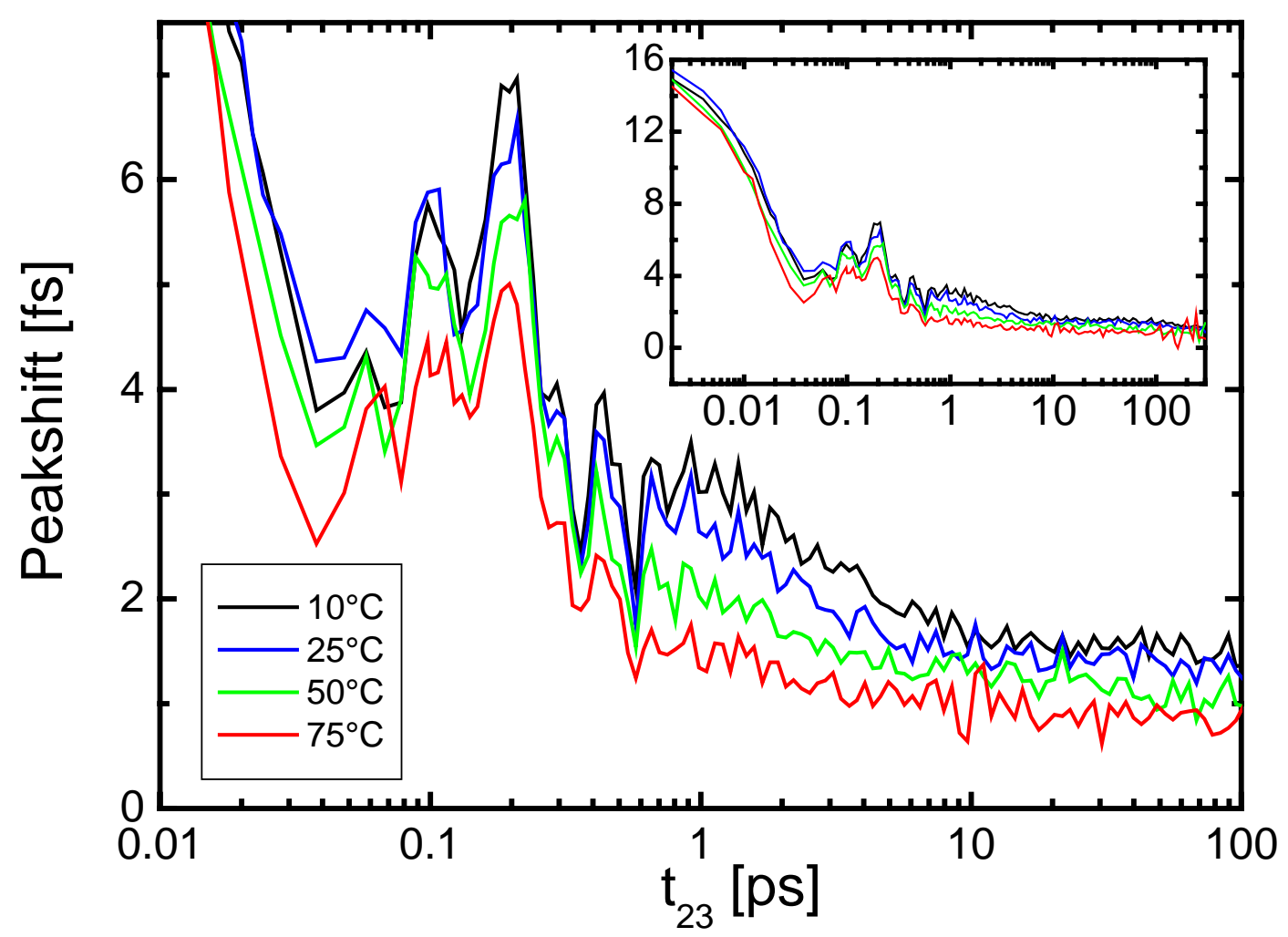

Abbildung 6.15: 3-PEPS-Funktion von DiDC $_{1}$ in Wasser bei den Temperaturen $10^{\circ} \mathrm{C}, 25^{\circ} \mathrm{C}, 50^{\circ} \mathrm{C}$ und $75^{\circ} \mathrm{C}$. Aufgetragen ist der Peakshift gegen die Zeit $\mathrm{t}_{23}$. In der Einblendung ist die gesamte Messkurve abgebildet, während im Hauptgraphen der Bereich vergrößert gezeigt ist, in dem Temperatureffekte sichtbar werden. 
Um das exponentielle Abklingverhalten richtig zu erfassen, wurden die Kurven in der Dreiecksgeometrie mit einem exponentiell anwachsenden Punktabstand gemessen. Es ist deutlich sichtbar, dass die Signale bei höheren Temperaturen schneller abklingen. Des Weiteren ist eine Inhomogenität bis hin zu 300 ps in der Einblendung zu beobachten.

Wie in Kapitel 6.3.1 beschrieben, wurden die gemessenen Daten mittels einer Gaußund dreier Exponentialfunktionen gemäß Gleichung 6.3 angepasst. Demnach ergeben sich die in Tabelle 6.2 zusammengetragenen Anpassungsparameter.

\begin{tabular}{|c|c|c|c|c|c|}
\hline Temperatur & $\mathbf{i}$ & $\mathbf{A}_{\mathrm{i}}$ & $\tau_{\mathbf{i}}$ & $\sigma$ & $\mathbf{A}_{\mathrm{i}} / \Sigma \mathbf{A}_{\mathbf{i}}$ \\
\hline \multirow{4}{*}{$10^{\circ} \mathrm{C}$} & 1 & $1.59 \mathrm{fs}$ & 700 ps & - & $10.2 \%$ \\
\hline & 2 & $0.38 \mathrm{fs}$ & $10 \mathrm{ps}$ & - & $2.4 \%$ \\
\hline & 3 & $2.65 \mathrm{fs}$ & 1.6 ps & - & $17.0 \%$ \\
\hline & 4 & $11 \mathrm{fs}$ & - & - & $70.4 \%$ \\
\hline \multirow{4}{*}{$25^{\circ} \mathrm{C}$} & 1 & $1.46 \mathrm{fs}$ & 750 ps & - & $9.1 \%$ \\
\hline & 2 & $0.35 \mathrm{fs}$ & 8 ps & - & $2.2 \%$ \\
\hline & 3 & $2.8 \mathrm{fs}$ & $1.1 \mathrm{ps}$ & - & $17.4 \%$ \\
\hline & 4 & $11.5 \mathrm{fs}$ & - & $11 \mathrm{fs}$ & $71.4 \%$ \\
\hline \multirow{4}{*}{$50^{\circ} \mathrm{C}$} & 1 & $1.18 \mathrm{fs}$ & $700 \mathrm{ps}$ & - & $7.7 \%$ \\
\hline & 2 & $0.4 \mathrm{fs}$ & 7 ps & - & $2.6 \%$ \\
\hline & 3 & $2.2 \mathrm{fs}$ & $0.8 \mathrm{ps}$ & - & $14.4 \%$ \\
\hline & 4 & $11.5 \mathrm{fs}$ & - & $11 \mathrm{fs}$ & $75.3 \%$ \\
\hline \multirow{4}{*}{$75^{\circ} \mathrm{C}$} & 1 & $1 \mathrm{fs}$ & 700 ps & - & $6.7 \%$ \\
\hline & 2 & $0.35 \mathrm{fs}$ & $3.5 \mathrm{ps}$ & - & $2.4 \%$ \\
\hline & 3 & $2 \mathrm{fs}$ & $0.45 \mathrm{ps}$ & - & $13.5 \%$ \\
\hline & 4 & 11.5 fs & & $11 \mathrm{fs}$ & $77.4 \%$ \\
\hline
\end{tabular}

Tabelle 6.2: Parameter der Anpassung der temperaturabhängigen Peakshiftkurven von DiDC $_{1}$ in Wasser mit den Gauß- und Exponentialfunktionen gemäß Gleichung 6.3.

Die Oszillationen des Farbstoffes lassen sich bei diesen Messkurven schlechter anpassen als bei den bereits vorgestellten 3-PEPS-Messungen in Wasser, da hier mit exponentiell wachsender Verzögerung gemessen wurde. Die Zeitkonstanten für $25^{\circ} \mathrm{C}$ stimmen gut mit denen aus der Boxcargeometrie (Tabelle 6.1) überein. 
Lediglich die Zeitkonstante für den quasi-statischen Teil der 3-PEPS-Kurve weicht deutlich ab. Dieser Unterschied auf längsten Zeitskalen resultiert, wie bereits erwähnt, aus einer unzureichenden Verzögerungszeit von $t_{23}$ und der Unschärfe des Zeitnullpunktes in der Boxcargeometrie. Auf diese langsame Abklingzeit wird daher nicht weiter eingegangen. Wie oben erläutert, werden im Folgenden lediglich die Zeitkonstanten $\tau_{2}$ und $\tau_{3}$ der 3-PEPS-Funktion diskutiert.

Diese beiden temperaturabhängigen Zeitkonstanten werden nun mit denen aus Messungen der dielektrischen Relaxation(DR), der magnetischen Kern-SpinResonanz (NMR), der zeitaufgelösten THz-Spektroskopie, des depolarisierten Ramanspektrums und des optischen Kerr-Effekts (OKE) verglichen.

Das Abklingverhalten der 3-PEPS-Kurve zwischen 3 bis 50 ps ist dominiert durch die Zeitkonstante $\tau_{2}=7.5 \mathrm{ps}$ bei $26^{\circ} \mathrm{C}$ und wurde bereits im vorherigen Abschnitt der Reorientierungskorrelationszeit individueller Wassermoleküle zugeordnet. Zum Vergleich dieser Zeitkonstanten mit denen aus anderen Messmethoden ermittelten Werten, ist es wichtig ihren Zusammenhang zu verwandten, experimentell zugänglichen Observablen zu kennen. In Photon-Echo-Experimenten, der dielektrischen Relaxation sowie der zeitaufgelösten THz-Spektroskopie wird die Zeitkonstante aus der Dipolkorrelationsfunktion bestimmt:

$$
\tau^{(1)}=\int_{0}^{\infty}\left\langle P_{1}\left[\sum_{j} \mu_{i}(0) \cdot \mu_{j}(t)\right]\right\rangle d t,
$$

\section{Gleichung 6.7}

wobei $\mu_{\mathrm{i}}$ das Dipolmoment des Moleküls i zum Zeitpunkt $t$ ist und $\mathrm{P}_{1}$ das erste Legendre-Polynom bezeichnet.

Demgegenüber liefern kernresonanz-spektroskopische Messungen der Spin-GitterRelaxationszeit die Orientierungskorrelationsfunktion:

$$
\tau^{(2)}=\int_{0}^{\infty}\left\langle\mathrm{P}_{2}\left[\mathrm{u}_{\mathrm{i}}(0) \cdot \mathrm{u}_{\mathrm{i}}(\mathrm{t})\right]\right\rangle \mathrm{dt} .
$$

\section{Gleichung 6.8}


Dabei beschreibt $\mathrm{u}_{\mathrm{i}}(\mathrm{t})$ einen Einheitsvektor im Molekül i zur Zeit $\mathrm{t}$ und $\mathrm{P}_{2}$ das zweite Legendre-Polynom.

Ein Vergleich der Relaxationszeiten aus Gleichung $6.7\left(\tau^{(1)}\right)$ und Gleichung $6.8\left(\tau^{(2)}\right)$ ist nur möglich, wenn bei der Dipolkorrelationsfunktion (Gleichung 6.7) die kollektiven Dynamiken verschwinden, d.h. alle Terme mit $\mathrm{i} \neq \mathrm{j}$ gleich Null sind. Lynden-Bell und Steele [172-174] zeigten, dass dann folgender Zusammenhang zwischen $\tau^{(1)}, \tau^{(2)}$ und der Debye-Relaxation $\tau_{D}$ gilt:

$$
\tau^{(2)}=\frac{\tau^{(1)}}{3}=\frac{\tau_{\mathrm{D}}}{3}
$$

\section{Gleichung 6.9}

Vöhringer und Mitarbeitern [150] konnten zeigen, dass dieser Zusammenhang zwischen $\tau^{(1)}$ und $\tau^{(2)}$ über den gesamten thermodynamisch Stabilitätsbereich der Flüssigkeit bei 1 bar Gültigkeit besitzt. Darüber hinaus konnte auch die langsamte Komponente der OKE-Signale eindeutig der Rotationsdiffusion individueller Moleküle zugeordnet werden. Die Autoren haben sämtliche temperaturabhängige Zeitkonstanten, die mit verschiedensten Techniken gewonnen wurden, zusammengetragen. Die Ergebnisse dieser Arbeit [150] sind in Abbildung 6.16 wiedergegeben. Zum Vergleich sind die temperaturabhängigen Zeitkonstanten der hier vorgestellten 3-PEPS-Experimente eingefügt. Die gute Übereinstimmung aller Daten bestätigt die ursprüngliche Zuordnung der 3-PEPS-Relaxationszeit $\tau_{2}$ zur diffusiven Reorientierung individueller Moleküle.

Die temperaturabhängige Zeitkonstante $\tau_{3}$ der 3-PEPS-Experimente, die im Bereich um 1 ps liegt, wurde übergedämpften, gehinderten kollektiven Translationsmoden sowie höherfrequenten Lösungsmittelmoden zugeordnet. In früheren Arbeiten wurde diese Zeitkonstante der mittleren Lebensdauer von Wasserstoffbrückenbindungen zugeschrieben. Aus einer Arrhenius-Auftragung kann in diesem Fall die Aktivierungsenergie und die Bindungsstärke bestimmt werden [175, 176]. Vöhringer und Mitarbeiter [150] konnten jedoch zeigen, dass diese Interpretation aus verschiedenen Gründen nicht zutreffend ist und die Relaxationszeit vielmehr gehinderte, kollektive Translationsmoden des Wassers repräsentiert. Das Verhältnis der Zeitkonstanten von $\mathrm{D}_{2} \mathrm{O} z u \mathrm{H}_{2} \mathrm{O}$ zeigt einen sehr geringen Isotopeneffekt und unterstützt die Interpretation einer kollektiven Translation [177]. Das temperatur- 
abhängige Verhalten der 3-PEPS-Signale bestätigt somit die bereits erfolgte Zuordnung der Zeitkonstanten.
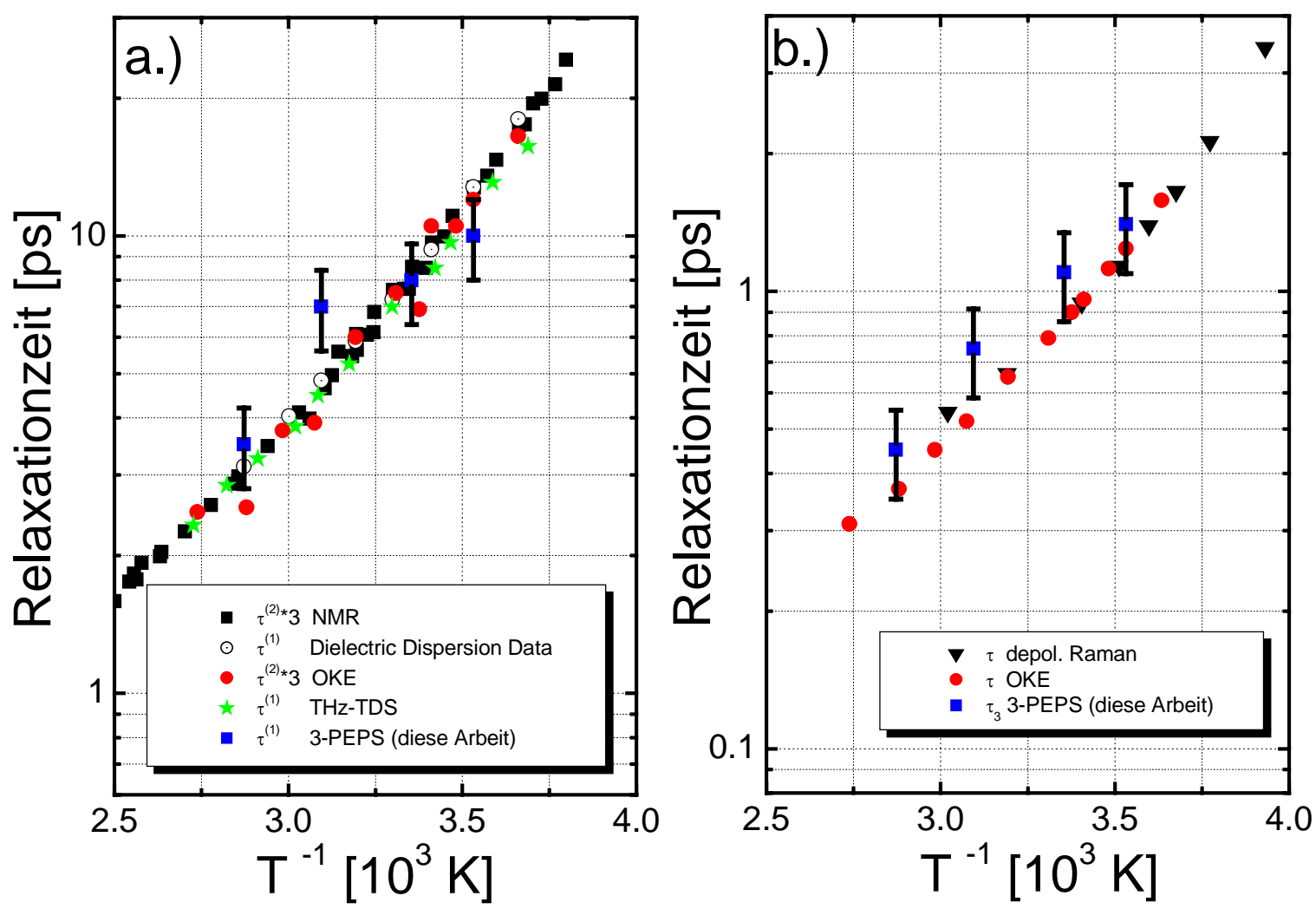

Abbildung 6.16: Zeitkonstanten der temperaturabhängigen Messungen von DiDC $_{1}$ in Wasser im Vergleich mit Zeitkonstanten aus der DR, NMR, OKE, THz und depolarisierten Ramanspektroskopie; Die Zeiten aus OKE- und NMR-Experimenten in Auftragung a.) wurden mit 3 multipliziert.

a.) Zeitkonstanten $\tau_{2}$ der 3-PEPS-Messungen mit Fehlerbalken

b.) Zeitkonstanten $\tau_{3}$ der 3-PEPS-Messungen mit Fehlerbalken 


\subsection{Drei-Puls-Echo-Peakshift-Messungen von DiDC $_{18}$ an der Lipidmembran-Wasser-Grenzschicht.}

Die Solvatationsdynamik der Wassermoleküle an der Lipidmembran-WasserGrenzschicht ist aus verschiedenen Gründen, auf die weiter unten eingegangen wird, nur unzureichend beschrieben. Um diese Dynamik zu bestimmen, wurde mit $\operatorname{DiDC}_{18}$ ein Farbstoff gewählt, der durch die langen aliphatischen Ketten und zwitterionischen Chromophor in den amphiphilen Eigenschaften den Lipiden ähnelt. Der Chromophor befindet sich daher direkt an der Lipidmembran-Wasser-Grenzschicht (Abbildung 6.1). Aus diesem Grund ist eine Solvatationsdynamik zu erwarten, die vom Wasser an der Grenzschicht und den Kopfgruppen der Lipide geprägt ist. Die Kopfgruppen lassen entsprechend ihrer Größe eine langsame Dynamik erwarten, während die Dynamik des reinen Wassers Zeitkonstanten im Bereich von wenigen Femto- bis hin zu einigen Pikosekunden aufweisen sollte. In diesem Zeitfenster sind die vorgestellten 3-PEPS-Messungen besonders empfindlich.

In Kapitel 6.4.1 wird die Solvatationsfunktion bei $26^{\circ} \mathrm{C}$ in DMPC analysiert. Die erhaltenen Ergebnisse werden in Kapitel 6.4.2 in Bezug auf Ergebnisse aus der Literatur diskutiert. Kapitel 6.4.3 konzentriert sich auf die Temperaturabhängigkeit dieser Funktion und hebt das Phasenverhalten der Vesikel und der damit verbundenen Solvatationsdynamik heraus.

\subsubsection{Analyse der Solvatationsdynamiken an der Lipidmembran-Wasser- Grenzschicht im Vergleich zum Wasser}

Die 3-PEPS-Kurve von $\mathrm{DiDC}_{18}$ an der Lipidmembran-Wasser-Grenzschicht ist in Abbildung 6.17 dargestellt. Auf kurzen Zeitskalen ( $\mathrm{t}_{23}<50 \mathrm{fs}$ ) klingt sie ähnlich wie in der wässrigen Volumenphase gaußförmig ab. Im Gegensatz zur Solvatationsdynamik des gleichen Chromophors in Wasser benötigt man nur zwei Exponentialfunktionen, um das Abklingen des 3-PEPS zu reproduzieren.

Die Zeitkonstante $\tau_{1}$ der langsamsten Komponente ist wie bei den Messungen im Wassers unzureichend bestimmbar. 

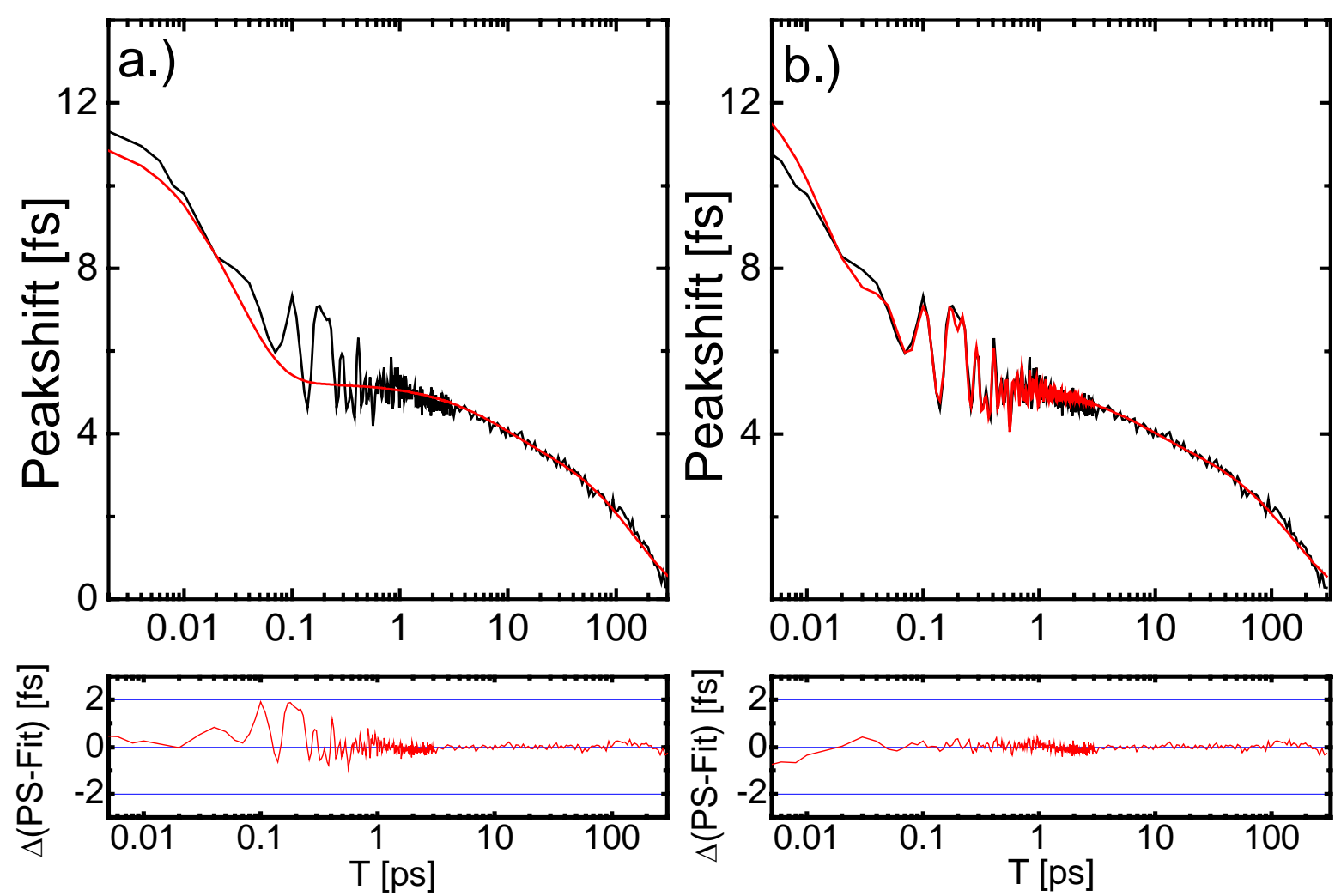

Abbildung 6.17: 3-PEPS-Kurven und Residuen von $\mathrm{DiDC}_{18}$ in DMPC/Wasser aufgetragen gegen die Verzögerungszeit $t_{23}$ inklusive der Residuen:

a.) Anpassungsfunktion bestehend aus drei Exponentialfunktionen;

b.) Anpassungsfunktion bestehend aus drei Exponentialfunktionen und gedämpften Schwingungen.

\begin{tabular}{|c|c|c|c|}
\hline $\mathbf{i}$ & $\mathbf{A}_{\mathbf{i}}$ & $\tau_{\mathbf{i}}$ & $\mathbf{A}_{\mathbf{i}} / \Sigma \mathbf{A}_{\mathbf{i}}$ \\
\hline 1 & $3.98 \mathrm{fs}$ & $155 \mathrm{ps}$ & $35.4 \%$ \\
\hline 2 & $1.25 \mathrm{fs}$ & $7.5 \mathrm{ps}$ & $11.1 \%$ \\
\hline 3 & - & - & - \\
\hline 4 & $6 \mathrm{fs}$ & $30 \mathrm{fs}$ & $53.4 \%$ \\
\hline
\end{tabular}

Tabelle 6.3: Zusammenstellung der ermittelten Parameter gemäß Gleichung 6.3; $\mathrm{DiDC}_{18}$ in DMPC/ $\mathrm{H}_{2} \mathrm{O}$

Erste Messungen an der Lipidmembran-Wasser-Grenzschicht in der Dreiecksgeometrie (nicht abgebildet) zeigen jedoch, dass der Peakshift bei 300 ps noch nicht auf Null abgefallen ist. Diese „Inhomogenität“ besitzt nahezu die doppelte Amplitude, 
wie die diskutierte "Inhomogenität" in der Wasservolumenphase. Bei den Grenzschichtmessungen reflektieren diese statischen Beiträge die großen Zeitskalen der Rotations- und Translationsdiffusion des Chromophors und entsprechen daher echten spektralen Diffusionsprozessen. Messungen der Stokes-Verschiebung geeigneter Chromophore in Zeitbereichen von mehreren Nanosekunden sollten in der Lage sein, diese langsame Dynamik näher zu charakterisieren.

Die Komponente mit der Zeitkonstanten $\tau_{2}$ dominiert nahezu das gesamte Abklingen der experimentellen Peakshift-Daten. Sie ist mit $\tau_{2}=\mathbf{7 . 5} \mathbf{p s}$ identisch zur bereits in Wasser beobachteten Rotationsdiffusion individueller Moleküle. Da der verwendete Chromophor derselbe wie bei den Messungen in reinem Wasser ist und sich außerdem in der polaren Grenzschicht mit hohem Wasseranteil befindet, ist es naheliegend, diese Relaxation Dynamiken einzelner Wassermoleküle zuzuschreiben. Die Relaxation angrenzender Kopfgruppen sollte dagegen auf längeren Zeitskalen stattfinden und trägt dementsprechend zur quasi-statischen Inhomogenität bei. Eine Komponente mit einer Abklingzeit von 1 ps wird im Gegensatz zu Wasser an der Grenzschicht nicht beobachtet. Das Fehlen dieser Zeitkonstanten, die den kollektiven gehinderten Translationsmoden des reinen Wassers zugeordnet wird, deutet auf eine Änderung der wasserspezifischen Struktur hin. Die kollektiven Translationsmoden stehen im direkten Zusammenhang zur Struktur des Wasserstoffbrückennetzwerkes. Eine vollständige Unterdrückung dieser Mode deutet auf ein stark gestörtes Wasserstoffbrückennetzwerk hin. Ganz offensichtlich ist das Wasserstoffbrückennetzwerk an der Lipidgrenzschicht stark durch die polaren Kopfgruppen gestört.

Eine Frequenzanalyse entsprechend Kapitel 6.3.1 ergibt die in Abbildung 6.17 b.) gezeigte Anpassung an die 3-PEPS-Daten. Das Rauschen ist, wie die Residuen in Abbildung 6.17 b.) zeigen, im Bereich von $\triangle P S= \pm 0.5$ fs.

Das Fourierspektrum ist in Abbildung 6.18 b.) dargestellt, während in Abbildung 6.18 a.) ein korrespondierendes TG-Spektrum abgebildet ist. Niederfrequente Anteile unterhalb $80 \mathrm{~cm}^{-1}$ sind wiederum subtrahiert. Beide Spektren stimmen sehr gut überein. Die Zuordnung der Schwingungsstruktur wurde bereits in Kapitel 6.1 d.) diskutiert. Abweichungen der Amplituden resultieren vermutlich aus unterschiedlichen Pulsdauern. 


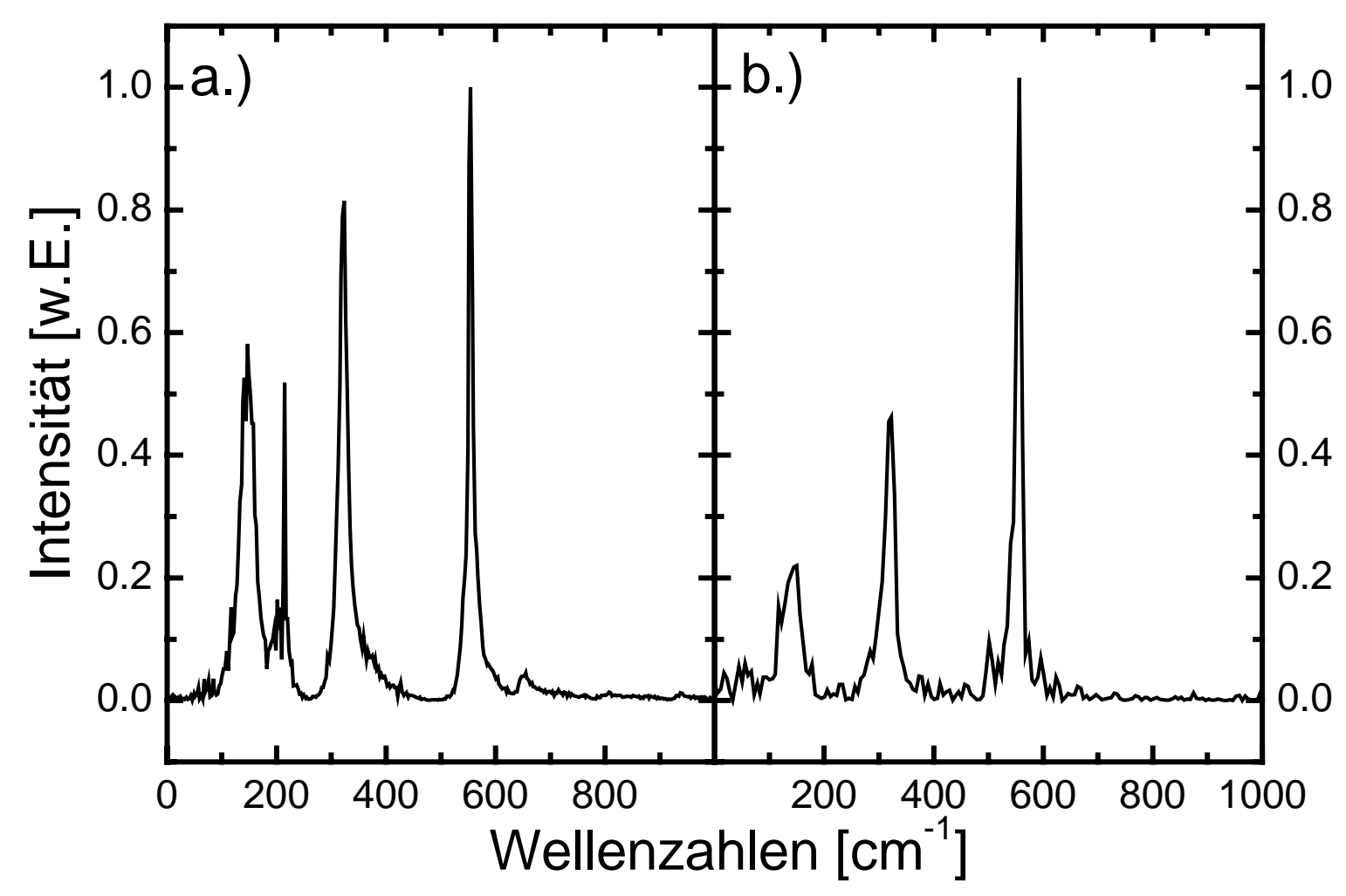

Abbildung 6.18: Fourierspektrum von $\mathrm{DiDC}_{18}$ in $\mathrm{DMPC} /$ Wasser bei $26^{\circ} \mathrm{C}$; Schwingungen unterhalb $80 \mathrm{~cm}^{-1}$ sind in beiden Graphen herausgefiltert, um einen besseren Vergleich der Spektren zu ermöglichen: a.) TG-Messung; b.) 3-PEPS-Messung.

\subsubsection{Diskusion der gemessenen Solvatationsdynamik in Bezug zu Arbeiten aus der Literatur}

Untersuchungen zur Solvatationsdynamik an der Lipidmembran-Wasser-Grenzschicht existieren zur Zeit nicht. Es sind dagegen Arbeiten zur Solvataionsdynamik in inneren Wassereinschlüssen von Lipidvesikeln und inversen Mizellen vorhanden [4, 5, 7, 10]. Zum einen reichte die Zeitauflösung der durchgeführten Experimente oftmals nicht aus, um die ultraschnelle Solvatationsdynamik des Wassers zu bestimmen, zum anderen waren die photochemischen Eigenschaften und die Einbaulagen der verwendeten Farbstoffe nur unzureichend bestimmt.

In der Literatur wurden eine Vielzahl von Farbstoffen zur Klärung der Solvatationsdynamik von Wasser in Lipidsystemen herangezogen, wobei im folgenden Abschnitt Untersuchungen präsentiert werden, welche die Farbstoffe Prodan, Laurdan, Patman, DCM, Coumarin 480 und Coumarin 343 als Sensor-Farbstoff verwenden. Die Strukturformeln dieser Fluoreszenzfarbstoffe sind in Abbildung 6.19 dargestellt. 
Sie zeichnen sich durch eine starke umgebungsspezifische Stokes-Verschiebung aus, welche aus der Änderung des Dipolmoments bei elektronischer Anregung herrührt.

\section{Prodan}

Laurdan

Patman<smiles>CCC(=O)c1ccc2cc(N(C)C)ccc2c1</smiles><smiles>CCCCCCCCCCCC(=O)c1ccc2cc(N(C)C)ccc2c1</smiles><smiles>CCCCCCCCCCCCCCC(=O)c1ccc2cc(N(C)CC[N+](C)(C)C)ccc2c1</smiles><smiles>Cc1cc(=O)oc2c3c4c(cc12)CCCN4CCC3</smiles><smiles></smiles>

Coumarin 480

Coumarin 343<smiles>CC1=CC(=C(C#N)C#N)C=C(C=Cc2ccc(N(C)C)cc2)O1</smiles>

\section{Abbildung 6.19: Zur Untersuchung der Solvatationsdynamik von Wasser in Lipidsystemen} verwendete Sensor-Farbstoffe.

Das von Weber und Mitarbeitern [178] synthetisierte und durch seine stationären Spektren in unterschiedlichen Lösungsmitteln charakterisierte Prodan weist eine Stokes-Verschiebung von $4302 \mathrm{~cm}^{-1}$ in Cyclohexan bis hin zu $8640 \mathrm{~cm}^{-1}$ in Wasser auf. Die Abhängigkeit der Stokes-Verschiebung vom Lösungsmittel wird auf dessen Orientierungspolarisation zurückgeführt. Ein protisches Lösungsmittel bewirkt bei gleicher Orientierungspolarisation eine größere Stokes-Verschiebung. Diese 
umgebungsspezifische Stokes-Verschiebung veranlasste Untersuchungen in biologischen Umgebungen mit Hilfe des Sensor-Farbstoffes Prodan. Erste Messungen in DMPC-Vesikeln wurden von Chong und Mitarbeitern [179, 180] durchgeführt. Diese stationären Fluoreszenzmessungen deuten darauf hin, dass sich der Farbstoff in den Bereich der Lipidmembran-Wasser-Grenzschicht einlagert. Von Sommer und Mitarbeitern [181] wurde die Solvatationsdynamik in künstlichen Lipiddoppelschichten bestimmt. Die zeitabhängige Stokes-Verschiebung wurde mit Mehrfrequenzphasen- und Mehrfrequenzmodulationsfluorometrie ermittelt, deren Zeitauflösung einige 100 ps beträgt. Auf Grund der limitierten Zeitauflösung beschränken sich die gemessenen Daten auf die Dynamik von Ketten und die Kopfgruppen der Lipide, während die Solvatationsdynamik des Wassers nicht aufgelöst werden konnte.

Die stationären Fluoreszenzspektren von Prodan in Membranen weisen zwei Maxima auf. Dies wirkt sich nachteilig auf die Bestimmung der Solvatationsdynamik aus. Ein solches Spektrum rührt vermutlich von zwei unterschiedlichen Einbaulagen in der Lipiddoppelschicht her $[179,180,182]$. Hinzu kommt eine geringe Wasserlöslichkeit von Prodan [178]. Eine von Krasnowska und Mitarbeitern [183] vorgeschlagene Interpretation des strukturierten Emissionspektrums schreibt das niederfrequente Maximum des Fluoreszenzspektrums den im Wasser gelösten Prodan zu. Eine andere Erklärungsmöglichkeit für die ausgeprägte bimodale Fluoreszenz offenbart sich durch Vergleich mit der Farbstoff-Sonde Laurdan. Diese besitzt einen zum Prodan identischen Chromophor und unterscheidet sich von letzterem lediglich in einer langen aliphatischen Kette. Auch beim Laurdan wird ein bimodales Fluoreszenzspektrun beobachtet. Dieses wird auf einen zusätzlich, höher energetischen Ladungstransferzustand $[12,13,209]$ zurückgeführt. Ein anderer Ansatz führt das bimodale Spektrum darauf zurück, dass die Umgebungssolvatation auf Zeitskalen der Fluoreszenzlebensdauer stattfindet [187, 188].

Beim Laurdan deuten die stationären Fluoreszenzspektren aufgrund der größeren Stokes-Verschiebung auf eine im Vergleich zum Prodan weniger polare Umgebung hin. Die von Viard und Mitarbeitern [185] ermittelten Dynamiken mit einer Zeitauflösung von ca. 500 ps werden daher den aliphatischen Ketten und Teilen der Kopfgruppe zugeordnet. Fluoreszenzanregungsspektren von Bagatolli und Mitarbeitern [187] deuten darauf hin, dass Laurdan an der Grenzfläche AlkylketteKopfgruppe eingelagert ist. 
Bei Patman handelt es sich um einen weiteren Farbstoff mit einem im Vergleich zum Prodan fast identischen Chromophor. Im Unterschied zu Prodan besitzt Patman eine lange aliphatische Kette ähnlich des Laurdans und eine Trimethylamoniummethylgruppe (Abbildung 6.19). Diese positiv geladene Gruppe soll eine Ausrichtung des Chromophors in Richtung der Lipidmembran-Wasser-Grenzschicht bewirken [1], während die aliphatische Kette den Chromophor in der Doppelschicht verankert.

Patman weist ebenso wie Prodan und Laurdan ein bimodales Emissionsspektrum auf, dessen Ursache nicht eindeutig bekannt ist. Des Weiteren deuten zeitaufgelöste Stokes-Verschiebungsmessungen [2] auf eine im Vergleich zu Prodan unpolarere Umgebung hin. Eine im Vergleich zu Prodan langsamere Solvatationsdynamik und eine kleinere stationäre Stokes-Verschiebung der Emission unterstützen eine solche Interpretation. Der Chromophor befindet sich demnach tiefer in der Lipiddoppelschicht als Prodan.

Die bisher durchgeführten Messungen mit den Sensor-Farbstoffen Prodan, Laurdan und Patman konnten die Solvatationsdynamik des Wassers nicht bestimmen, da bei einer Zeitauflösung der dynamischen Messungen von > 100 ps vorwiegend die Orientierung der aliphatischen Ketten, die Dynamik der Lipidkopfgruppen und die Dynamik des Farbstoffes selbst gemessen wird. Des Weiteren sind die Prozesse, welche zu den bimodalen Fluoreszenzspektren führen nicht ausreichend verstanden. Ein weiterer Farbstoff, der zur Messung der Solvatationsdynamik in Lipiden eingesetzt wurde, ist Coumarin 480. Dieser Farbstoff ist in Wasser unlöslich. In Messungen zur dynamischen Stokes-Verschiebung mit einer Zeitauflösung von 50 ps konnten zwei Zeitkonstanten der Solvatation bestimmt werden [3]. Diese Zeitkonstanten wurden der Dynamik in inneren Wassereinschlüssen der Vesikel zugeordnet.

Vergleichbare Messungen mit dem Sensor-Farbstoff DCM in Vesikeln bzw. Mizellen [5-8] ergeben ebenfalls, dass sich die Solvatation durch Wasser mit zwei Zeitkonstanten beschreiben lässt. Das Solvatationsverhalten des Wassers wird in einen schnellen Anteil mit einer Zeitkonstanten von 0.5 ns bis 1 ns und in einen langsamen Anteil mit einer Zeitkonstanten von 2 ns bis 11 ns gegliedert. Die Messungen wurden mit einer Zeitauflösung von ca. 50 ps durchgeführt. Ein Großteil der Solvatationsdynamik blieb auf Grund dieser Zeitauflösung unbeobachtet.

Von Levinger und Mitarbeitern [10] wurden vergleichbare Experimente in inversen 
Mizellen mit Coumarin $\mathbf{3 4 3}$ mit Hilfe der Fluoreszenzkonvertierungsspektroskopie durchgeführt. Mit dieser Technik wurde eine Zeitauflösung von ca. 160 fs erreicht. Es war daher möglich, die Solvatation in inversen Mizellen mit der des reinen Wassers zu vergleichen [11]. Die ermittelten Relaxationszeiten in inversen Mizellen sind bedeutend langsamer als in reinem Wasser. Des Weiteren wird entsprechend den obigen Experimenten mit DCM eine Abklingzeit auf einer Nanosekundenzeitskala beobachtet. Diese Zeitkonstante scheint ihre Ursache in der durch die Lipidschicht gestörten Umgebung zu haben. Nandi und Mitarbeiter haben diesen Zusammenhang herausgearbeitet und postulieren zwei unterschiedliche Formen des Wassers in der Lipidumgebung. Sie unterscheiden zwischen freiem Wasser, welches der reinen Wasserphase entspricht, und gebundenem Wasser. Dieses gebundene Wasser wird auch als „biologisches Wasser" bezeichnet und befindet sich im Bereich der Kopfgruppen. Ein möglicher dynamischer Austausch zwischen diesen Wasserspezies wird diskutiert $[9,12,188]$.

Es bleibt festzuhalten, dass den vorgestellten Sensor-Farbstoffen DCM und den Coumarinen 343 und 480 keine definierte Einbaulage bezüglich der Grenzschicht zugeordnet werden konnte. Außerdem sind lediglich Fluoreszenzkonvertierungsmessungen in der Lage die Solvatationsdynamiken von reinem Wasser mit ausreichender Zeitauflösung zu bestimmen.

Die in der vorliegenden Arbeit vorgestellten 3-PEPS-Experimente besitzen zum einen die notwendige Zeitauflösung um die Solvatationsdynamik in Wasser zu beschreiben, zum anderen ist der Farbstoff definiert in der Lipidmembran-WasserGrenzschicht verankert. Die langsamen Zeitkonstanten könnten in den bei den 3-PEPS-Messungen gefundenen quasi-statischen Inhomogenitäten verborgen sein. Die Ursache dieser langsamen Zeitkonstanten bleibt jedoch weiterhin unklar, da für einen Vergleich mit der Solvatationsdynamik in reinem Wasser die experimentellen Ergebnisse auf diesen langen Zeitskalen noch nicht vorhanden sind. Es kann somit nicht unterschieden werden, ob das Modell von Nandi und Mitarbeitern von freiem und gebundenen Wasser zutrifft oder vielmehr kollektive Moden des Wassers für diese langsamen Zeiten verantwortlich sind.

In Bezug auf die Kurzzeitdynamik der vorgestellten 3-PEPS-Messungen geben molekulardynamische Berechnungen einen weiteren Einblick in die Struktur des Wassers an der Grenzschicht. Aus diesen Berechnungen geht hervor, dass zwei bis drei Hydrathüllen um die Kopfgruppen der Lipide angeordnet sind [64]. Die 
Wassermoleküle sind über Wasserstoffbrücken an das Sauerstoffatom der Phosphatgruppen und an den Stickstoff der Lipidkopfgruppe als Klathrate gebunden $[64,65]$. Die Dynamik des Wassers an der Lipidmembran-WasserGrenzschicht zeigt deutliche Veränderungen im Vergleich zum reinen Wasser. Des Weiteren weisen die Dipole des Wassers in Richtung des Membraninneren [66]. In Übereinstimmung mit den 3-PEPS-Messungen dieser Arbeit wird das Wasserstoffbrückennetzwerk von den Lipidkopfgruppen stark beeinflusst, so dass es zu einer Eliminierung der kollektiven Translationsfreiheitsgrade kommt. Eine freie Diffusion der Wassermoleküle findet dennoch statt. Die Reorientierung scheint im gleichen Maße wie im reinen Wasser zur Solvatation beizutragen [66]. Diese aus molekulardynamischen Simulationen erhaltenen Ergebnisse decken sich mit den hier vorgestellten Resultaten aus 3-PEPS-Experimenten.

\subsubsection{Temperaturabhängigkeit der Solvatationsdynamik an der Lipidmembran- Wasser-Grenzschicht}

Die in Kapitel 6.4.1 vorgestellte Messung gibt über das Solvatationsverhalten an der Grenzschicht in der flüssig-kristallinen Phase Auskunft. Diese Phase ist von biologischer Relevanz und daher auch am intensivsten untersucht. Die Phasenübergänge und die Veränderungen an der Grenzschicht geben jedoch einen tieferen Einblick in die Wechselwirkungen der Lipide untereinander, die letztendlich zur Ausbildung einer Doppelschicht führen. Alle Funktionen der Membran bauen auf diesen Wechselwirkungen auf. Aus diesem Grund wurde das Solvatationsverhalten auch in den biologisch weniger relevanten zackigen und gelförmigen Phasen bestimmt.

Bei der Beschreibung der 3-PEPS-Kurven wird bei den folgenden Messungen auf eine erneute Betrachtung der oszillatorischen Bestandteile verzichtet, da diese bereits im vorherigen Abschnitt diskutiert wurden. Ebenso findet der ultraschnelle Abfall der 3-PEPS-Kurve keine weitere Beachtung, da die 3-PEPS-Experimente in diesem Zeitbereich nicht im direkten Zusammenhang zur Solvatationskorrelationsfunktion stehen. 


\subsubsection{Temperaturabhängigkeit der Solvatationsdynamik an der DMPC- Wasser-Grenzschicht}

Abbildung 6.20 zeigt eine temperaturabhängige Wärmekapazitätsmessung von DMPC-Vesikeln. Die Pfeile kennzeichnen Temperaturen, bei denen 3-PEPSMessungen durchgeführt wurden. Die Messung in der gelförmigen Phase $L_{\beta}$, wurde bei $6^{\circ} \mathrm{C}$ durchgeführt und die Messung in der zackigen Phase $P_{\beta}$, in der Nähe des Vorphasenübergangs bei $11^{\circ} \mathrm{C}$. Weiterhin wurde in der flüssig-kristallinen Phase $\mathrm{L}_{\alpha}$ bei $26^{\circ} \mathrm{C}$ und $30^{\circ} \mathrm{C}$ gemessen. Es existieren also Messungen in allen drei thermodynamischen Phasen von DMPC-Vesikeln, die oberhalb der Schmelztemperatur von Wasser bekannt sind.

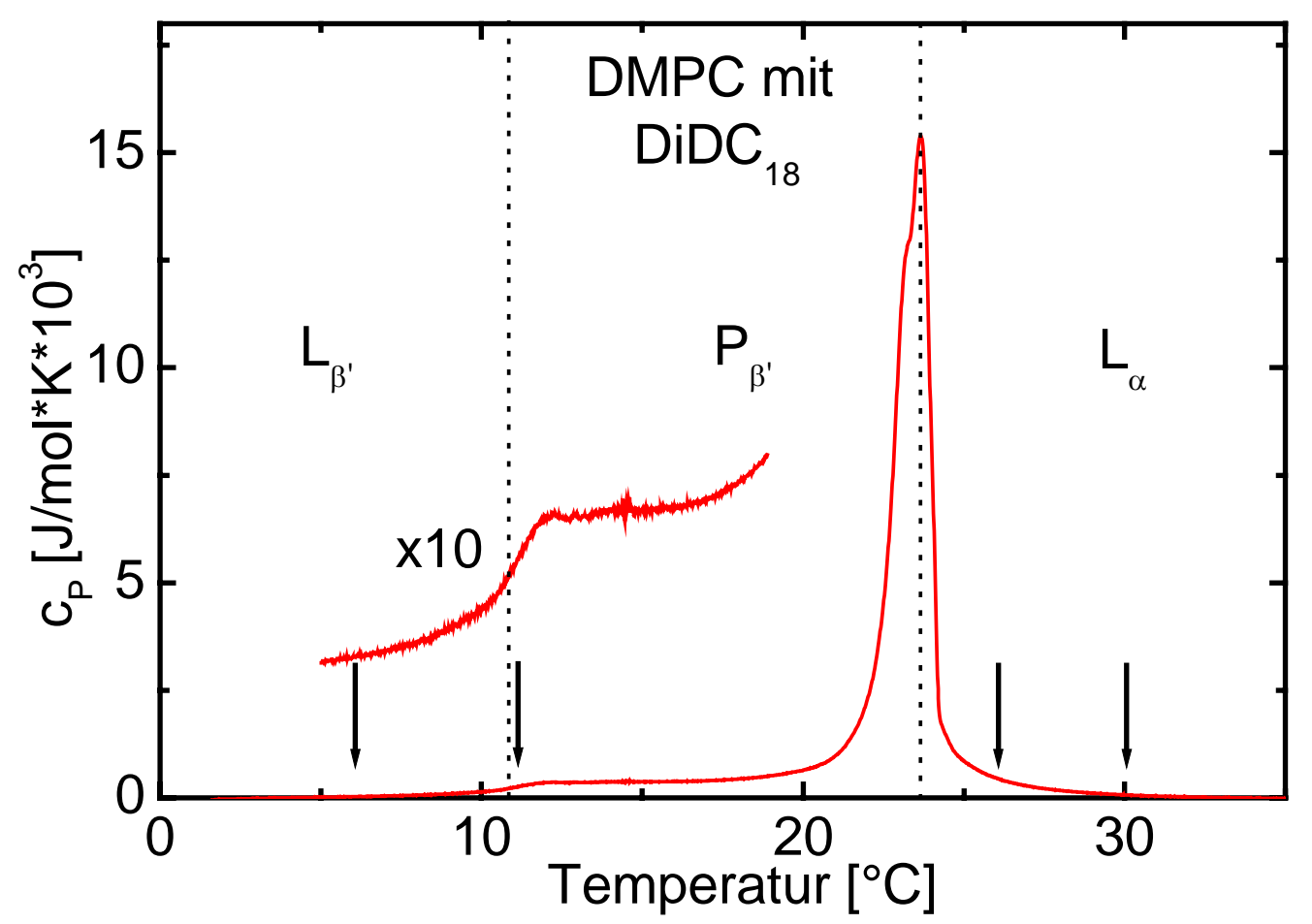

Abbildung 6.20: Temperaturabhängige Wärmekapazität von DMPC-Vesikeln; mit Pfeilen sind die Temperaturen der durchgeführten 3-PEPS-Experimenten gekennzeichnet.

Die 3-PEPS-Messungen bei diesen Temperaturen sind in Abbildung 6.21 dargestellt. Jede Messkurve wurde mit einer Summe aus zwei Exponentialfunktionen angepasst ( $i=1-2$; gestrichelte Kurve), bei $6^{\circ} \mathrm{C}$ ist zusätzlich eine Summe dreier exponentieller Komponenten notwendig ( $i=1-3$; breite durchgezogene Linie). Die Abklingzeiten und die jeweiligen Amplituden der Exponentialfunktionen sind Tabelle 6.4 zu entnehmen. 


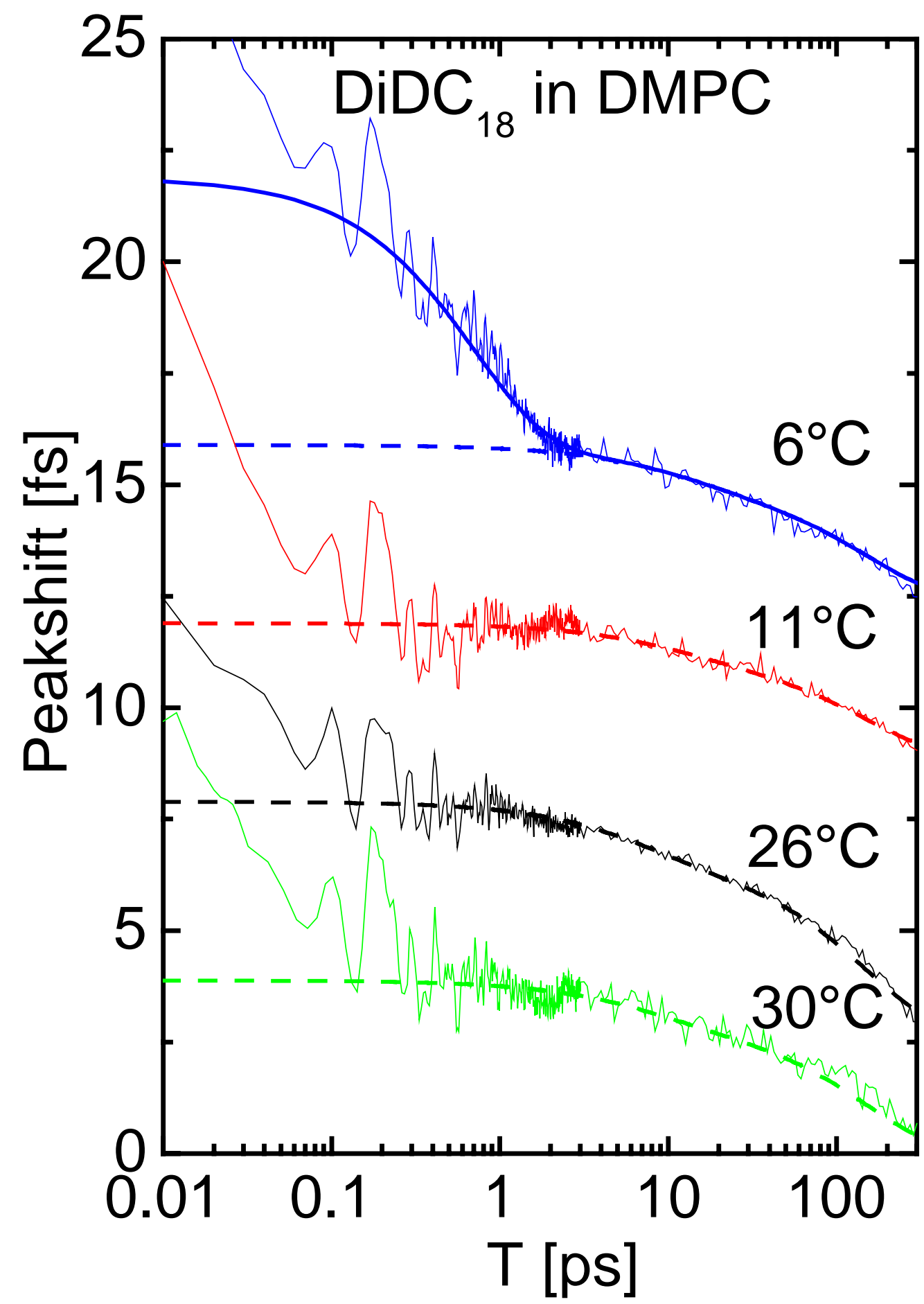

Abbildung 6.21: 3-PEPS-Kurven von $\mathrm{DiDC}_{18}$ in DMPC/Wasser bei den Temperaturen $6^{\circ} \mathrm{C}, 11^{\circ} \mathrm{C}$, $26^{\circ} \mathrm{C}$ und $30^{\circ} \mathrm{C}$. Zur besseren Veranschaulichung sind die jeweiligen Messungen relativ zueinander verschoben. Die gestrichelten Kurven geben eine Anpassung mit zwei Exponentialfunktionen wieder, die durchgezogene Linie entspricht einer Anpassung bei $6^{\circ} \mathrm{C}$, die drei Exponentialfunktionen beinhaltet. 


\begin{tabular}{|c|c|c|c|c|}
\hline Temperatur & Lipidphase & $\mathbf{i}$ & $\mathbf{A}_{\mathbf{i}}$ & $\tau_{\mathbf{i}}$ \\
\hline \multirow{2}{*}{$\mathbf{6}^{\circ} \mathbf{C}$} & \multirow{2}{*}{ gelförmig $\mathrm{L}_{\beta^{\prime}}$} & 2 & $0.8 \mathrm{fs}$ & $12 \mathrm{ps}$ \\
& & 3 & $6 \mathrm{fs}$ & $0.7 \mathrm{ps}$ \\
\hline \multirow{2}{*}{$\mathbf{1 1}^{\circ} \mathbf{C}$} & zackig $\mathrm{P}_{\beta}$, & 1 & $2.3 \mathrm{fs}$ & $150 \mathrm{ps}$ \\
& & 2 & $0.7 \mathrm{fs}$ & $11 \mathrm{ps}$ \\
\hline \multirow{2}{*}{$\mathbf{2 6}^{\circ} \mathbf{C}$} & flüssig-kristallin $\mathrm{L}_{\alpha}$ & 2 & $1.25 \mathrm{fs}$ & $7.5 \mathrm{ps}$ \\
& & 1 & $2.98 \mathrm{fs}$ & $150 \mathrm{ps}$ \\
\hline \multirow{2}{*}{$\mathbf{3 0}^{\circ} \mathbf{C}$} & flüssig-kristallin $\mathrm{L}_{\alpha}$ & 2 & $0.9 \mathrm{fs}$ & $8 \mathrm{ps}$ \\
& & $2.98 \mathrm{fs}$ & $155 \mathrm{ps}$ \\
\hline
\end{tabular}

Tabelle 6.4: Parameter zur Anpassung der temperaturabhängigen 3-PEPS-Signale von DiDC $_{18}$ in DMPC gemäß Gleichung 6.3.

Die Langzeitkomponente $\tau_{1}$ liegt in allen Messungen bei etwa 150 ps. Eine genaue Zuordnung ist, wie oben bereits mehrfach diskutiert, nicht möglich. Die Zeitkonstante $\tau_{2}$ wird der Rotationsdiffusion individueller Wassermoleküle zugeordnet, deren Relaxationszeit mehrere Pikosekunden beträgt. Dieser Freiheitsgrad der Wassermoleküle ist somit in allen Phasen gleichermaßen an der Solvatation beteiligt.

Wie bereits erwähnt, mußte die 3-PEPS-Kurve der gelförmigen Phase mit einer dritten exponentiellen Komponenete angepasst werden. Zusätzlich zu den Zeitkonstanten im Pikosekundenbereich erscheint eine Relaxationszeit von $\tau_{3}=700$ fs, die mit einer großen Amplitude einhergeht. Es ist durchaus erstaunlich, dass sich die Solvatationsdynamik beim Übergang von der zackigen Phase $\mathrm{P}_{\beta}$, zur gelförmigen Phase $L_{\beta}$, derart drastisch ändert. Demgegenüber bleibt der Peakshift durch den Hauptphasenübergang unverändert. Es stellt sich daher die Frage, ob diese neue Zeitkonstante spezifisch für die gelförmige Phase ist und ob das Auftreten dieser dritten Zeitkonstanten mit strukturellen Änderungen am Vorphasenübergang verknüpft ist. Bemerkenswerterweise ist die 3-PEPS-Messung in der gelförmigen Phase sehr ähnlich zu der in wässrigen Volumenphasen (siehe Abbildung 6.11). Zur Klärung dieses Sachverhalts werden Messungen an weiteren Membranen vorgestellt. 


\subsubsection{Temperaturabhängigkeit der Solvatationsdynamik an der DOPC- Wasser-Grenzschicht}

Analog zu Kapitel 6.4.3.1 wird nun die Temperaturabhängigkeit von DiDC $_{18}$ in DOPC beschrieben. Abbildung 6.22 zeigt die 3-PEPS-Messungen bei $6^{\circ} \mathrm{C}, 11^{\circ} \mathrm{C}$ und $20^{\circ} \mathrm{C}$ in doppelt und einfach logarithmierter Darstellung. Die Hauptphasenübergangstemperatur von DOPC liegt bei $-18^{\circ} \mathrm{C}$, so dass sich DOPC bei allen drei Messungen in der flüssig-kristallinen Phase befindet. Die dargestellten 3-PEPS-Kurven sind mit einer Funktion angepasst, die sich aus der Summe zweier Exponentialfunktionen zusammensetzt. Die Zeitkonstante für den langsamen, quasi-statischen Anteil der Solvatationsfunktion beträgt 400 ps, während der größte Anteil der verbleibenden 3-PEPS-Kurve mit einer Zeitkonstante von 9 ps angepasst werden kann. Die Relaxationszeit von 9 ps entspricht auch hier der Rotationsdiffusion individueller Wassermoleküle.
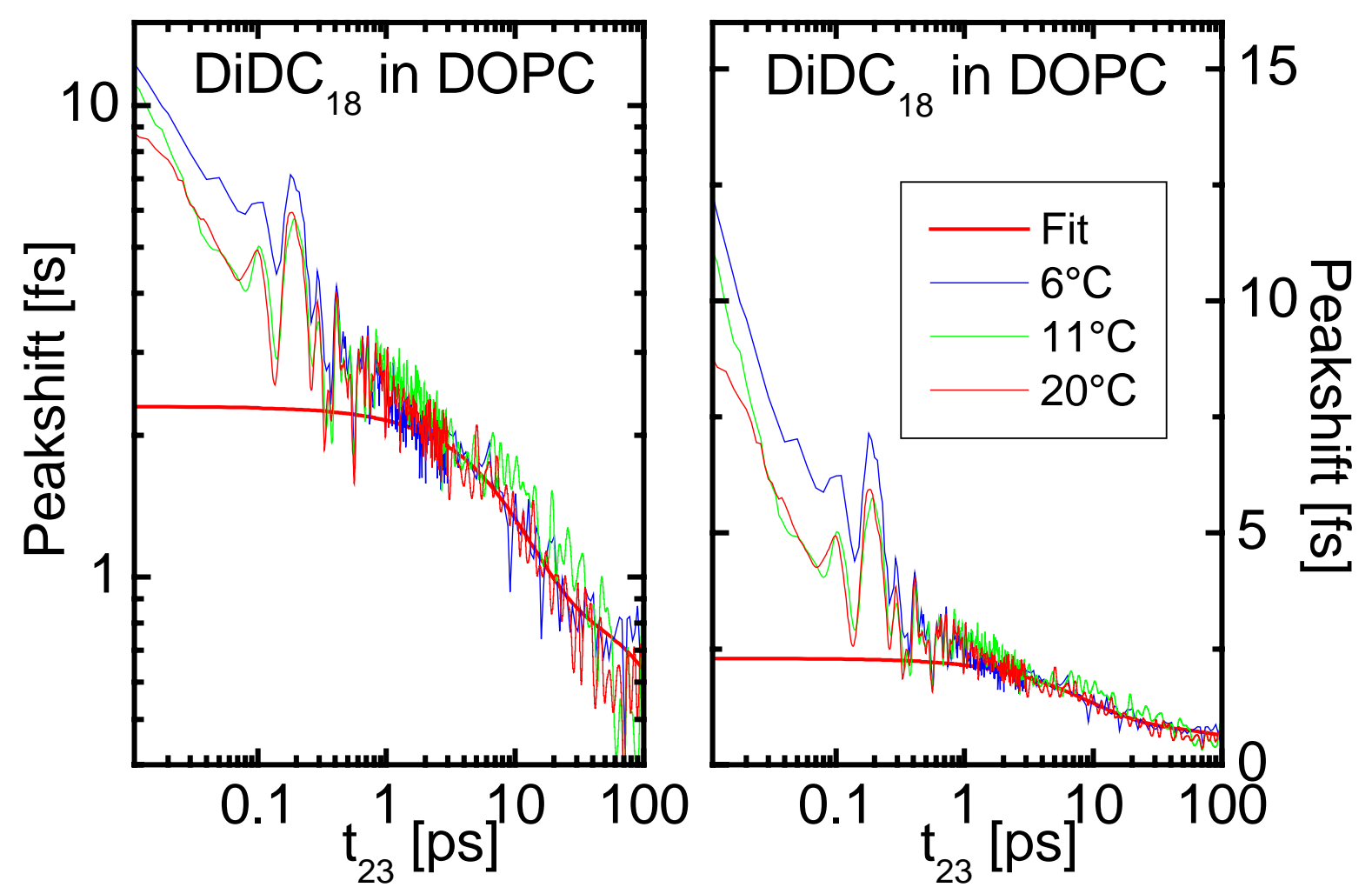

Abbildung 6.22: 3-PEPS-Kurven von DiDC $_{18}$ in DOPC/Wasser bei den Temperaturen $6^{\circ} \mathrm{C}, 11^{\circ} \mathrm{C}$ und $20^{\circ} \mathrm{C}$ inklusive einer biexponentiellen Anpassungsfunktion 


\begin{tabular}{|c|c|c|}
\hline $\mathbf{i}$ & $\mathbf{A}_{\mathbf{i}}$ & $\tau_{\mathbf{i}}$ \\
\hline 1 & $1.2 \mathrm{fs}$ & $400 \mathrm{ps}$ \\
\hline 2 & $1.4 \mathrm{fs}$ & $9 \mathrm{ps}$ \\
\hline
\end{tabular}

Tabelle 6.5: Zusammenstellung der ermittelten Parameter gemäß Gleichung 6.3 von $\mathrm{DiDC}_{18}$ in DOPC/Wasser.

Diese Messungen zeigen, dass in der flüssig-kristallinen Phase des DOPCs der Freiheitsgrad der Rotationsdiffusion individueller Wassermoleküle zur Solvatation beiträgt, jedoch keine kollektiven Translationsmoden vorhanden sind. Diese Ergebnis deckt sich mit denen aus der flüssig-kristallinen Phase des DMPC. Dieser Befund unterstreicht, dass die Struktur des Wassers an der Grenzschicht von PhospholipidVesikeln in der flüssig-kristallinen Phase sich stark von der des reinen Wassers unterscheidet. Diese Messungen zeigen darüber hinaus, dass keine ausgeprägten Veränderungen der Solvatationsdynamik bei einer Temperaturänderung von $6^{\circ} \mathrm{C}$ auf $11^{\circ} \mathrm{C}$ bei DOPC-Vesikeln stattfindet. Die Ursache für die Änderung der Solvatationsdynamik bei DMPC-Vesikeln ist somit bei der sich wandelnden Struktur am Vorphasenübergang zu suchen. In diesem Fall sollten auch bei anderen Lipidsorten deutliche Veränderungen am Vorphasenübergang auftreten. Aus diesem Grund wird die Solvatationsdynamik an der Grenzschicht von DPPC-Vesikeln zu Wasser untersucht.

\subsubsection{Temperaturabhängigkeit der Solvatationsdynamik an der DPPC- Wasser-Grenzschicht}

Temperaturabhängige 3-PEPS-Messungen wurden an dem System $\mathrm{DiDC}_{18} /$ DPPC/Wasser bei $6^{\circ} \mathrm{C}, 20^{\circ} \mathrm{C}, 30^{\circ} \mathrm{C}$ und $35^{\circ} \mathrm{C}$ durchgeführt. In Abbildung 6.23 ist eine Wärmekapazitätsmessung von DPPC-Vesikeln, die mit dem Farbstoff DiDC 18 markiert sind, dargestellt. Mit den Pfeilen sind die Temperaturen bei denen die verschiedenen 3-PEPS-Messung durchgeführt wurden gekennzeichnet. Bei den Messungen bei $6^{\circ} \mathrm{C}, 20^{\circ} \mathrm{C}$ und $30^{\circ} \mathrm{C}$ befinden sich die DPPC-Vesikel demnach in der gelförmigen Phase $\mathrm{L}_{\beta}$, während die Messung bei $35^{\circ} \mathrm{C}$ die Solvatationsdynamik an der Grenzschicht der DPPC-Vesikel in der zackigen Phase $\mathrm{P}_{\beta}$, bestimmt. 


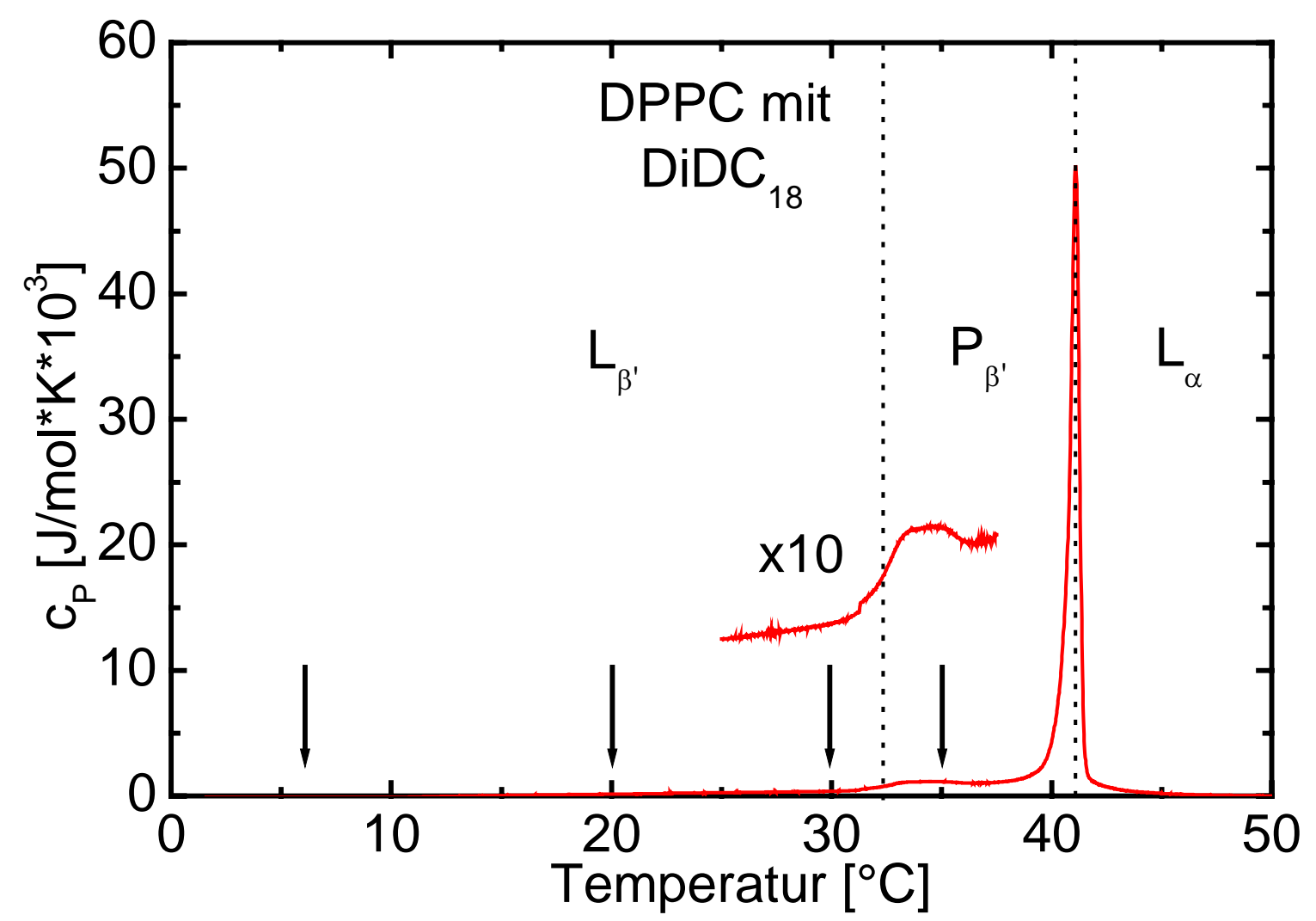

Abbildung 6.23: Temperaturabhängige Wärmekapazität von DPPC-Vesikeln; mit Pfeilen sind die Temperaturen der durchgeführten 3-PEPS-Experimenten gekennzeichnet.

Die Messkurven der 3-PEPS-Experimente sind in Abbildung 6.24 aufgetragen. Für die Anpassung der Solvatationsdynamik in der gelförmigen Phase sind jeweils drei exponentiell abklingende Terme der Summe in Gleichung 6.3 notwendig, während zwei ausreichen, um die Dynamik in der zackigen Phase zu beschreiben. Die Amplituden $A_{i}$ und Abklingzeiten $\tau_{i}$ der Anpassungsfunktion I $\left(t_{23}\right)$ sind in der Tabelle 6.6 zusammengestellt.

Wie auch bei den beiden zuvor beschriebenen Systemen beobachtet werden konnte, tritt bei $\mathrm{DiDC}_{18}$ in DPPC-Vesikeln eine exponentielle Langzeitkomponente von $\tau_{1}=160 \mathrm{ps}$ auf, die aus den bereits diskutierten Gründen (Boxcargeometrie) fehlerbehaftet ist.

Die Zeitkonstante von $\tau_{2}=11$ ps wird gemäß vorheriger Diskussion der Messungen an den Grenzschichten von DMPC- und DOPC-Vesikeln der Rotationsdiffusion individueller Wassermoleküle zugeschrieben. 


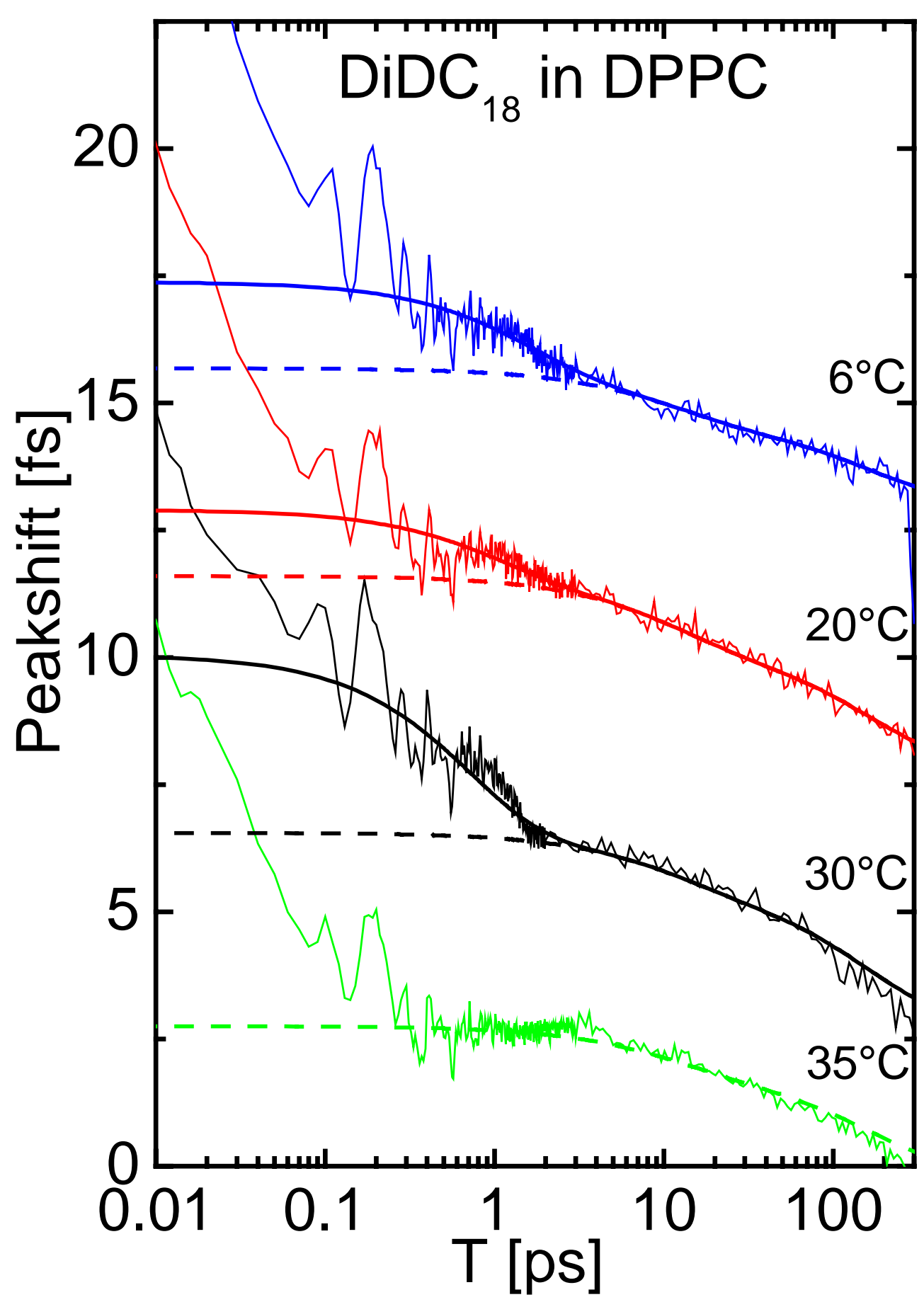

Abbildung 6.24: 3-PEPS-Kurven an der Grenzschicht von DPPC-Vesikeln zu Wasser bei den Temperaturen $6^{\circ} \mathrm{C}, 20^{\circ} \mathrm{C}, 30^{\circ} \mathrm{C}$ und $35^{\circ} \mathrm{C}$. Die einzelnen Messungen sind der Übersichtlichkeit wegen in ihrem Peakshift relativ zueinander verschoben. Die gestrichelt gezeichneten Kurven geben eine biexponentielle Anpassung wieder, während die durchgezogen gezeichnete Linie einer triexponentielle Anpassung entspricht. 


\begin{tabular}{|c|c|c|c|c|}
\hline Temperatur & Lipidphase & $\mathbf{i}$ & $\mathbf{A}_{\mathrm{i}}$ & $\tau_{\mathbf{i}}$ \\
\hline $6^{\circ} \mathrm{C}$ & gelförmig $L_{\beta}$, & $\begin{array}{l}1 \\
2 \\
3\end{array}$ & $\begin{array}{c}1.55 \mathrm{fs} \\
1 \mathrm{fs} \\
1.7 \mathrm{fs}\end{array}$ & $\begin{array}{c}160 \mathrm{ps} \\
11 \mathrm{ps} \\
1.5 \mathrm{ps}\end{array}$ \\
\hline $20^{\circ} \mathrm{C}$ & gelförmig $L_{\beta}$, & $\begin{array}{l}1 \\
2 \\
3\end{array}$ & $\begin{array}{l}2.3 \mathrm{fs} \\
1.3 \mathrm{fs} \\
1.3 \mathrm{fs}\end{array}$ & $\begin{array}{c}160 \mathrm{ps} \\
11 \mathrm{ps} \\
1 \mathrm{ps}\end{array}$ \\
\hline $30^{\circ} \mathrm{C}$ & gelförmig $\mathrm{L}_{\beta}$, & $\begin{array}{l}1 \\
2 \\
3\end{array}$ & $\begin{array}{c}2.65 \text { fs } \\
1 \text { fs } \\
3.5 \text { fs }\end{array}$ & $\begin{array}{c}160 \text { ps } \\
11 \text { ps } \\
0.7 \text { ps }\end{array}$ \\
\hline $35^{\circ} \mathrm{C}$ & zackig $\mathrm{P}_{\beta}$, & $\begin{array}{l}1 \\
2\end{array}$ & $\begin{array}{c}1.9 \text { fs } \\
0.85 \text { fs }\end{array}$ & $\begin{array}{c}160 \text { ps } \\
11 \text { ps }\end{array}$ \\
\hline
\end{tabular}

Tabelle 6.6: Parameter zur Anpassung der temperaturabhängigen 3-PEPS-Signale von DiDC $_{18}$ in DPPC/Wasser gemäß Gleichung 6.3

Interessanterweise tritt auch in diesem System eine Zeitkonstante um 1 ps in der gelförmigen Phase auf. Die Zusammenstellung der Messungen in DMPC, DOPC und DPPC belegt deutlich, dass das Auftreten dieser Zeitkonstanten im Zusammenhang mit dem Vorphasenübergang stehen muss (Kapitel 3.3.4). Obwohl viele Fragen bezüglich des Vorphasenübergangs noch ungeklärt sind, ist trotzdem eindeutig belegt, dass er mit einer Veränderung der Kopfgruppenanordnung einhergeht. Dies ist daher mit der Abnahme der Hydratisierung beim Übergang von der zackigen Phase $P_{\beta}$, zur gelförmigen Phase $L_{\beta}$, (Kapitel 3.3.6) verknüpft. Demgegenüber ist der Hauptphasenübergang mit dem „Schmelzen“ der aliphatischen Ketten verbunden. Wegen dieser unterschiedlichen strukturdynamischen Phänomene am Vorphasenund Hauptphasenübergang, ist es nicht erstaunlich, dass sich die Solvatationsdynamik an der Grenzschicht am Vorphasenübergang stärker ändert als am Hauptphasenübergang. Beim Vergleich der in diesem Kapitel vorgestellten Messungen mit denen in der wässrigen Volumenphase (Kapitel 6.3) treten folgende Ähnlichkeiten auf: 
Das temperaturabhängige Verhalten der schnellen Zeitkonstanten $\tau_{3}$ im Bereich von 1 ps ist bei DiDC $_{18}$ in DPPC vergleichbar mit dem in Wasser. Diese Zeitkonstanten wurden in die in Kapitel 6.3.2 verwendete Arrhenius-Auftragung (Abbildung 6.16 b.)) eingefügt.

Abbildung 6.25 stellt also die temperaturabhängigen Relaxationszeiten des Chromophors in Wasser und in DPPC/Wasser dar und vergleicht diese mit den Zeitkonstanten, die aus depolarisierten Raman-Daten und Messungen des optischen Kerr-Effektes der reinen Flüssigkeit ermittelt wurden. Die Abbildung zeigt eine gute Übereinstimmung aller Zeitkonstanten. Diese nur in der gelförmigen Phase auftretende Zeitkonstante kann somit den gehinderten kollektiven Translationsmoden des reinen Wassers zugeordnet werden.

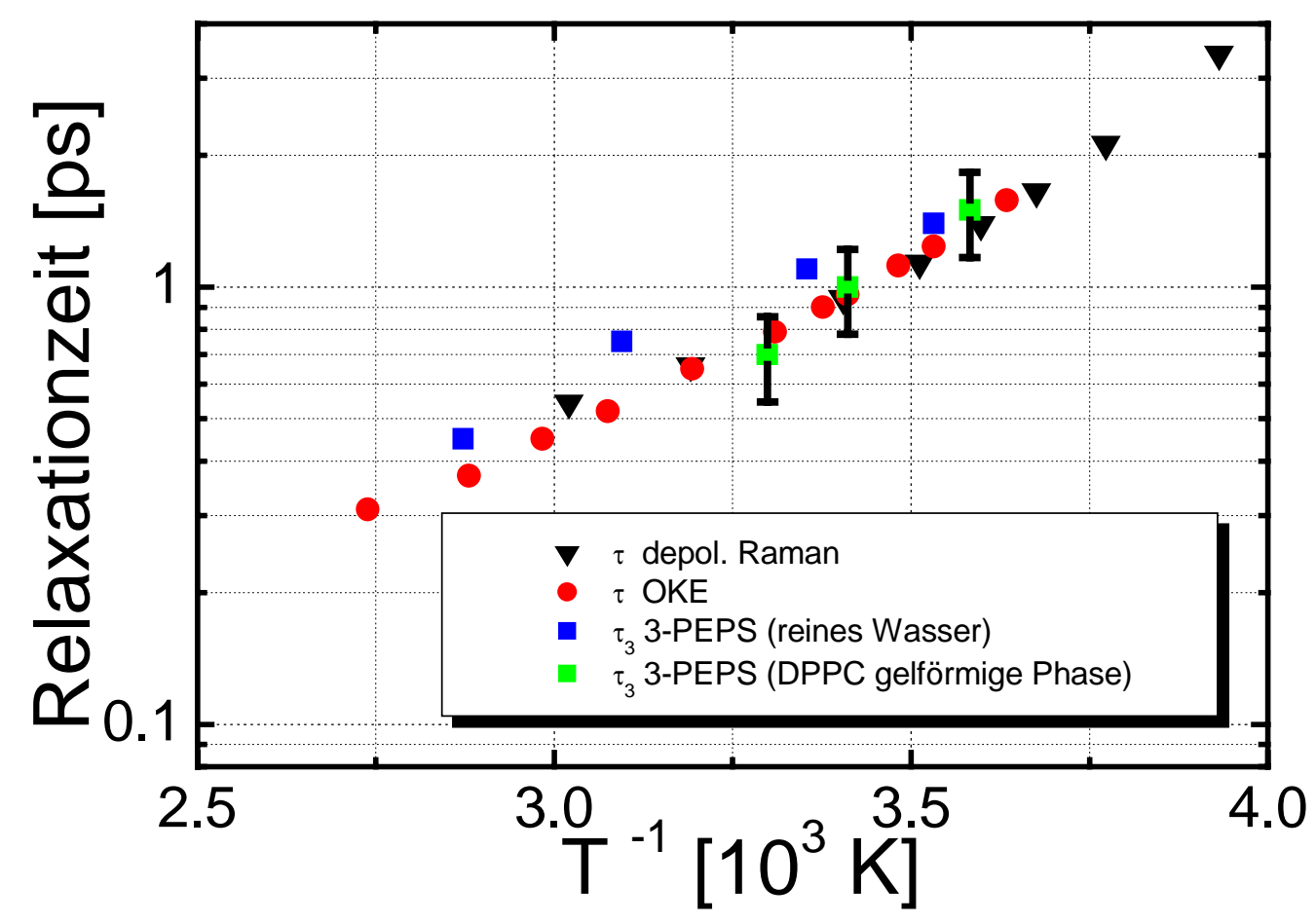

Abbildung 6.25: Temperaturabhängigkeit der Zeitkonstante $\tau_{3}$ bei Messungen in der gelförmigen Phase $L_{\beta}$, an der DPPC-Wasser-Grenzschicht kombiniert mit den Zeitkonstanten aus depolarisierten Raman- und OKE-Messungen.

Eine mögliche Erklärung für das Auftreten dieser weiteren Zeitkonstanten besteht in dem durch die Dehydratisierung hervorgerufenen „Herausdrängen“ des Chromophors aus dem Grenzschichtbereich. In diesem Fall würde der Farbstoff in den Bereich der Wasservolumenphase hineinragen und, wie in den Experimenten 
beobachtet wurde, als Sensor für die Solvatationsdynamik von reinem ungestörtem Wasser dienen. Trotzdem bleibt der Farbstoff weiterhin durch die langen aliphatischen Ketten in der Lipiddoppelschicht verankert. Unterstützt wird diese Annahme von der erhöhten Packungsdichte der gelförmigen Phase $L_{\beta}$, im Vergleich zur zackigen Phase $\mathrm{P}_{\beta}$. Diese Interpretation erklärt die Tatsache, dass eine sehr ähnliche Solvatationsdynamik in der zackigen und flüssig-kristallinen Phase vorliegt, da am Hauptphasenübergang keine Dehydratisierung sondern vielmehr ein Schmelzen der aliphatischen Ketten im Innern der Doppelschicht stattfindet. Der Chromophor befindet sich jedoch an den Kopfgruppen und ist somit, wie anhand der Messungen gezeigt werden konnte, nur für Veränderungen im Kopfgruppenbereich sensitiv. 


\section{Ausblick}

Die hier durchgeführten Experimente geben erste Einblicke in die Solvatation an Lipidmembran-Wasser-Grenzschichten. Die Isolation der grenzschicht-spezifischen Solvationsdynamik gelingt durch einen Vergleich mit der eines identischen Chromophors in wässriger Volumenphase. Im zuletzt genannten Fall entwickelt sich die Dynamik im Wesentlichen auf zwei Zeitskalen: etwa 1 ps für die Relaxation kollektiver gehinderter Translationsmoden, charakteristisch für das zufällige, ungestörte Wasserstoffbrückennetzwerk der reinen Flüssigkeit und etwa 10 ps für die diffusive Reorientierungsdynamik. Dagegen erfolgt die Solvatation an wässrigen Phospholipidmembran-Grenzschichten nur auf einer Zeitskala von mehreren Pikosekunden. Der Vergleich zeigt, dass das für reines Wasser charakteristische Wasserstoffbrückennetzwerk an der Oberfläche dieser Grenzschicht aufgehoben ist. Die ablaufenden ultraschnellen Dynamiken im Bereich der Lipidmembrane sind mit Ausnahme der in dieser Arbeit diskutierten Ergebnissen noch unverstanden. Es eröffnet sich daher ein völlig neues Forschungsgebiet.

Die Photon-Echo-Spektroskopie erlaubt im Zusammenspiel mit lokalisierten Farbstoffsensoren die Solvatation in allen Regionen der Membran zu untersuchen. Durch derartige Messungen können molekulardynamische Berechnungen überprüft und verfeinert werden.

Eine wichtige Funktion von biologischen Membranen ist die Trennung zwischen inneren und äußeren Regionen der Zelle. Ionen sind nicht in der Lage diese Barriere beliebig zu durchdringen. Lediglich durch lonenkanäle können lonen selektiv aus der Zelle heraus oder in die Zelle hinein gelangen. Mit Hilfe der Photon-EchoSpektroskopie könnten neue detaillierte Einblicke in die Dynamik dieses lonentransportes gewonnen werden. Die hohe Zeit- und Ortsauflösung macht diese Art der Spektroskopie zu einem brauchbaren Werkzeug, um die Solvatationsdynamik mittels eines Farbstoffs über den gesamten Bereich des lonenkanals zu messen.

In Bezug auf das gestörte Wasserstoffbrückennetzwerk ist es von besonderem Interesse eine Serie von Farbstoffen zu synthetisieren, die die Solvatationsdynamik im Bereich direkt an den Kopfgruppen bis hin zur Region des reinen ungestörten Wassers gezielt auflöst. Ein Phosphatidylcholinlipid, das einen kovalent an die Kopfgruppe gebundenen Chromophor besitzt, könnte hierzu eingesetzt werden. Das 
in dieser Arbeit verwendete Indocarbocyanin ist ein dafür gut geeigneter Chromophor, da er einen großen Extinktionskoeffizient besitzt. Der variable Abstand könnte durch eine variable aber starre Brücke, die über eine kovalente Bindung die Phosphatidylcholinkopfgruppe mit dem Farbstoff verknüpft, eingestellt werden.

Des Weiteren ist eine Verbesserung der Messtechnik wünschenswert, um die gesamte Solvatationskorrelationsfunktion analysieren zu können. Um dies umsetzen zu können ist es notwendig, einerseits den Inertialteil aufzulösen und andererseits die langen Zeitkonstanten im Bereich der Rotationskorrelationszeit des Farbstofffes und den kollektiven Rotationen von Wasser genauer zu bestimmen.

Die Probleme im Bereich der inertialen Umgebungsdynamik resultieren aus der fehlenden Sensitivität der 3-PEPS-Messung in Bezug auf den schnellen Anteil der Solvatationskorrelationsfunktion $\mathrm{M}_{\text {fast }}$ (siehe Kapitel 4.5). Ein Experiment, das zur Bestimmung dieser ultraschnellen Komponente bereits genutzt wird, ist das „timegated“ 3-Puls-Echo [75, 189]. Die Durchführung dieser Experimente in Küvetten wird jedoch erschwert, da das Echosignal durch das Austrittsfenster zeitlich dispergiert wird. Die bisherigen „time-gated“ 3-PE Experimente wurden in schnellfließenden Jets durchgeführt. Für biologische Systeme muss daher nach eine Methode gesucht werden, die trotz des kleinen Probenvolumens eine Messung des „time-gated“ 3-PE erlaubt.

Die Interpretation der Zeitkonstanten im Bereich von einigen 100 ps war in dieser Arbeit nicht möglich, da die Solvatationskorrelationsfunktion auf Grund des experimentellen Aufbaus lediglich bis zu einer Verzögerung $t_{23}=300$ ps gemessen werden konnte. Eine Verlängerung der Verzögerungsstrecke könnte helfen, diese langsamen Dynamiken zu beschreiben. Die maximale Verzögerungszeit für die 3-PEPS-Experiment wird entscheidend von der Fluoreszenzlebensdauer des eingesetzten Farbstoffes geprägt, da die Intensität des Signals mit der Zeitkonstante der Fluoreszenzlebensdauer abklingt. Des Weiteren ist es für die genaue Bestimmung dieser langsamen Zeitkonstante wichtig, den Peakshift möglichst genau zu bestimmen, da der Peakshift zu langen Zeiten nahe zu null ist. In diesem Zusammenhang ist es notwendig das Signal-Rauschverhältnis weiter zu verbessern. Neben den Optionen im Bereich der Lipidmembranen bietet sich die Photon-EchoSpektroskopie an, die Solvatationsdynamik auch in anderen biologischer System zu bestimmen. Mit den in dieser Arbeit verwendeten Methoden ist eine genaue Charakterisierung der Solvatationsfunktion für Zeiten zwischen 80 fs bis zu ungefähr 
50 ps möglich. Insbesondere die Rotationskorrelationszeit und die gehinderte kollektive Translation des Wassers konnte mit hoher Genauigkeit bestimmt werden. Experimente zur Bestimmung der Solvatationsdynamik des Wasser in anderen biologischen Bereichen bieten sich daher an. Einige Systeme wurden, wie bereits erwähnt, schon untersucht. Diesen Experimenten fehlte es jedoch häufig an einer guten Ortsauflösung der Solvatationsdynamik, da die Position des Farbstoffes nur ungenau bekannt war [7, 8]. Mit dem in diesen Experimenten benutzten Farbstoff, der sich durch seine langen Ketten an einem wohldefinierten Ort aufhält, sollte es möglich sein schon untersuchte Systeme, wie das Wasser in inversen Mizellen [7], noch genauer zu charakterisieren.

Ein biologisch relevantes Beispiel in dem Grenzschichten zu Wasser eine Rolle spielen sind Proteine. Die Funktion der Porteine, unter anderem der Einfluss der tertiären Struktur, werden zur Zeit intensiv untersucht. Um ihre Strukturen und damit die Wirkungsweise der Proteine zu verstehen, ist eine Charakterisierung des Wassers in den verschiedenen Bereichen des Proteins notwendig. Photon-EchoExperimente an markierten Proteinen in wässriger Lösung bieten sich auf Grund ihrer Ortsauflösung an. Die Dynamik des Protein umgebenden Wassers könnte auch in Abhängigkeit vom Zustand (gefaltet oder entfaltet) des Proteins untersucht werden.

Die in dieser Arbeit vorgestellten Experimente haben gezeigt, dass die Photon-EchoSpektroskopie ein Werkzeug zur Aufklärung von Solvatationsdynamiken in biologischen Grenzschichten ist. Ihre Möglichkeiten scheint zur Zeit lediglich durch die photchemischen und photophysikalischen Eigenschaften der zur Verfügung stehenden Farbstoffe begrenzt zu sein. 


\section{ANHANG}

\subsection{Synthese von 1,1 '-Dioctadecyl-3,3,3',3`- tetramethylindodicarbocyaninchlorid}

\subsubsection{4-Chlorobenzolsulfonsäureoctadecylester}

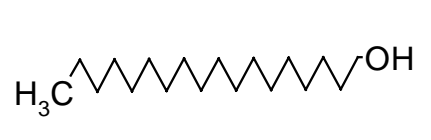

270,50

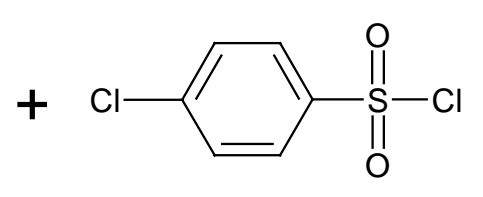

211,07

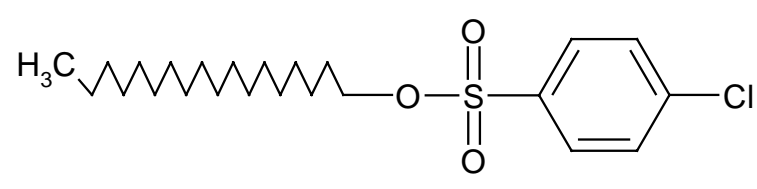

445,11

Edukte: $0.75 \mathrm{~mol}$ Stearylalkohol; $0.87 \mathrm{~mol}$ Triethylamin;

$1 \mathrm{~mol}$ p-Chlorbenzolsulfonsäurechlorid

\section{Durchführung:}

Eine Mischung aus $203 \mathrm{~g}$ (0.75 mol) Stearylalkohol, $120 \mathrm{ml}(0.87 \mathrm{~mol})$ Triethylamin und $800 \mathrm{ml}$ Dichlormethan wurde nach dem Erwärmen bis zur Klärung bei Zimmertemperatur unter Rühren mit einer Lösung von $211 \mathrm{~g}(1 \mathrm{Mol}) \mathrm{p}$-Chlorbenzolsulfonsäurechlorid tropfenweise versetzt.

Nach 24-stündigem Rühren wurde das Filtrat mehrmals mit Wasser gewaschen, über $\mathrm{Na}_{2} \mathrm{SO}_{4}$ getrocknet, filtriert und das Lösungsmittel entfernt.

$\mathrm{C}_{24} \mathrm{H}_{41} \mathrm{ClO}_{3} \mathrm{~S}(445.11)$

Fp.Lit.: $63-64^{\circ} \mathrm{C}$. 


\subsubsection{2,3,3-Trimethyl-1-octadecyl-3H-indolium-4-chlorbenzoat}<smiles>CC1(C)C(Cl)=Nc2ccccc21</smiles>

159,23

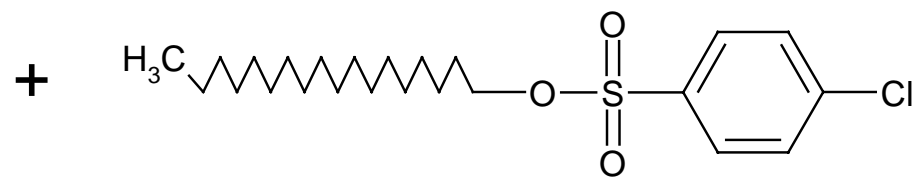

445,11

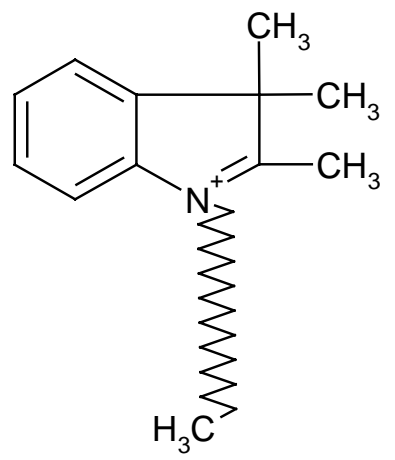

604,34

Edukte: $0.15 \mathrm{~mol}$ 2,3,3-Trimethyl-3H-indol;

$0.15 \mathrm{~mol} 4$-Chlorobenzolsulfonsäureoctadecylester

\section{Durchführung:}

$25 \mathrm{~g}$ (0.15 mol) 2,3,3-Trimethyl-3H-indol und $67 \mathrm{~g}$ (0.15 mol) 4-Chlorobenzolsulfonsäureoctadecylester wurden in einem $500 \mathrm{ml}$ Kolben unter Stickstoffatmosphäre 2 Stunden bei $220^{\circ} \mathrm{C}$ gerührt. Nach dem Abkühlen wurde das Reaktionsgemisch mit $250 \mathrm{ml}$ Ethylacetat versetzt. In violetter Lösung bildete sich ein weißer Niederschlag. Dieser wurde abfiltriert und in Ethylacetat gewaschen und anschließend aus $300 \mathrm{ml}$ Ethylacetat umkristallisiert.

$\mathrm{C}_{35} \mathrm{H}_{54} \mathrm{CINO}_{3} \mathrm{~S}(604.34)$

Fp.Lit.: $89-91^{\circ} \mathrm{C}$. 


\subsubsection{1,1`-Dioctadecyl-3,3,3`,3`-tetramethylindodicarbocyaninchlorid}
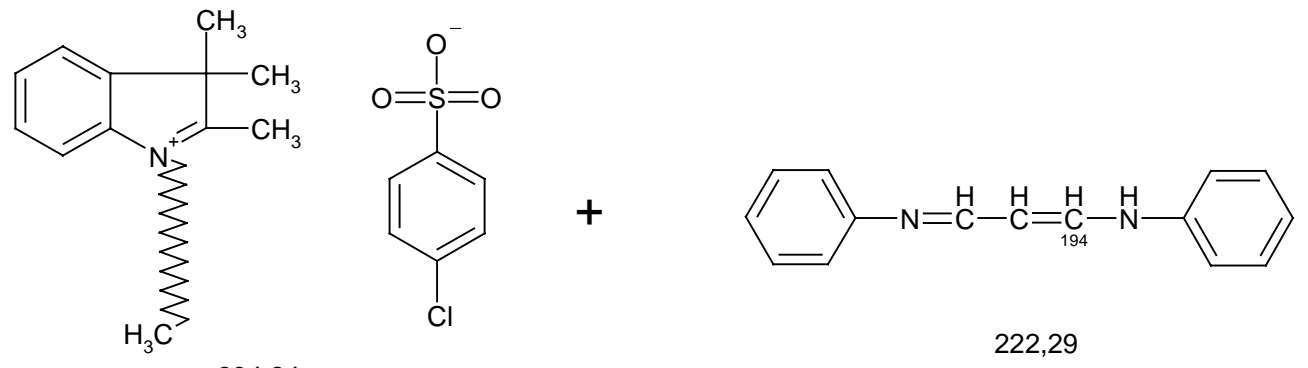

222,29

604,34

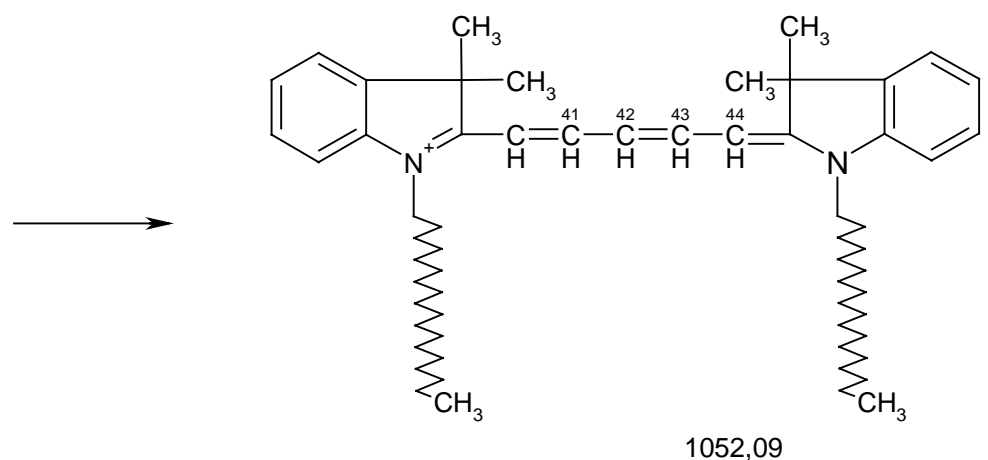

$\mathrm{Cl}^{-}$

Edukte: 0.02 mol 2,3,3-Trimethyl-1-octadecyl-3H-indolium-4-chlorbenzoat;

0.01 mol 3-Anilinoacrylaldehyde-anil

\section{Durchführung:}

$12.1 \mathrm{~g}$ (0.02 mol) 2,3,3-Trimethyl-1-octadecyl-3H-indolium-4-chlorbenzoat, $2.22 \mathrm{~g}$ (0.01 mol) 3-Anilinoacrylaldehyde-anil und $2 \mathrm{~g}$ Natriumacetat wurden in $60 \mathrm{ml}$ Essigsäureanhydrid 1 Stunde unter Rückfluss gerührt. Nach dem Abkühlen wurden $60 \mathrm{ml}$ Wasser zu dem Gemisch hinzugegeben und das Reaktionsgemisch wurde über Nacht stehengelassen. Es bildete sich ein zähes dunkles Produkt neben einer hellbrauner Wasserphase. Das Wasser wurde entfernt und der Rückstand in Wasser gewaschen. Anschließend wurde der Rückstand in $50 \mathrm{ml}$ 2-Propanol gelöst. Das tiefblaue Filtrat wurd abfiltriert und unter Vakuum getrocknet.

$\mathrm{C}_{61} \mathrm{H}_{99} \mathrm{ClN} 2$ (895.92). 


\section{LITERATURVERZEICHNIS}

[1] R. Hutterer, F. W. Schneider, H. Sprinz, M. Hof, Binding and Relaxation Behaviour of Prodan and Patman in Phospholipid Vesicles - a Fluorescence and ${ }^{1}$ H-NMR Study, Biophysical Chemistry 61 (1996) 151-160.

[2] R. Hutterer, A. B. J. Parusel, M. Hof, Solvent Relaxation of Prodan and Patman: A useful Tool for the Determination of Polarity and Rigidity Changes in Membranes, Journal of Fluorescence 8 (1998) 389-393.

[3] A. Datta, S. K. Pal, D. Mandal, K. Bhattacharyya, Solvation Dynamics of Coumarin 480 in Vesicles, Journal of Physical Chemistry B 102 (1998) 61146117.

[4] K. Bhattacharyya, B. Bagchi, Slow dynamics of constrained water in complex geometries [Review], Journal of Physical Chemistry 104 (2000) 10603-10613.

[5] S. K. Pal, D. Sukul, D. Mandal, S. Sen, K. Bhattacharyya, Solvation Dynamics of DCM in Dipalmitoyl Phosphatidylcholine Lipid, Tetrahedron 56 (2000) 69997002.

[6] S. K. Pal, D. Sukul, D. Mandal, K. Bhattacharyya, Solvation Dynamics of DCM in Lipid, Journal of Physical Chemistry B 104 (2000) 4529-4531.

[7] S. K. Pal, D. Sukul, D. Mandal, S. Sen, K. Bhattacharyya, Solvation Dynamics of DCM in Micelles, Chemical Physics Letters 327 (2000) 91-96.

[8] S. K. Pal, D. Mandal, D. Sukul, S. Sen, K. Bhattacharyya, Solvation Dynamics of DCM in Human Serum Albumin, Journal of Physical Chemistry B 105 (2001) 1438-1441.

[9] N. Nandi, K. Bhattacharyya, B. Bagchi, Dielectric Relaxation and Solvation Dynamics of Water in Complex Chemical and Biological Systems [Review], Chemical Reviews 100 (2000) 2013-2045.

[10] N. E. Levinger, Ultrafast Dynamics in Reverse Micelles, Microemulsions, and Vesicles, Current Opinion in Colloid \& Interface Science 5 (2000) 118-124.

[11] R. E. Riter, D. M. Willard, N. E. Levinger, Water Immobilization at Surfactant Interfaces in Reverse Micelles, Journal of Physical Chemistry B 102 (1998) 2705-2714.

[12] D. Pant, R. E. Riter, N. E. Levinger, Influence of Restricted Environment and lonic Interactions on Water Solvation Dynamics, Journal of Chemical Physics 109 (1998) 9995-10003. 
[13] G. Cevc, D. Marsh, Phospholipid Bilayers: Physical Principles and Models, John Wiley \& Sons, New York 1987.

[14] R. Dimova, C. Dietrich, A. Hadjiisky, K. Danov, B. Pouligny, Falling Ball Viscosimetry of Giant Vesicle Membranes: Finite-Size Effects, European Physical Journal B 12 (1999) 589-598.

[15] R. Dimova, B. Pouligny, C. Dietrich, Pretransitional Effects in Dimyristoylphosphatidylcholine Vesicle Membranes: Optical Dynamometry Study, Biophysical Journal 79 (2000) 340-356.

[16] D. G. Hunter, B. J. Frisken, Effect of Extrusion Pressure and Lipid Properties On the Size and Polydispersity of Lipid Vesicles, Biophysical Journal 74 (1998) 2996-3002.

[17] R. C. MacDonald, R. I. MacDonald, B. P. M. Menco, K. Takeshita, N. K. Subbarao, L.-R. Hu, Small-Volume Extrusion Apparatus for Preparation of Large Unilamellar Vesicles, Biochimica et Biophysica Acta 1061 (1991) 297303.

[18] F. Bordi, C. Cametti, A. Naglieri, Ionic Transport in Lipid Bilayer Membranes, Biophysical Journal 74 (1998) 1358-1370.

[19] A. Srivastava, K. B. Eisenthal, Kinetics of Molecular Transport Across a Liposome Bilayer, Chemical Physics Letters 292 (1998) 345-351.

[20] A. Jutila, P. K. J. Kinnunen, Novel Features of the Main Transition of Dimyristoylphosphocholine Bilayers Revealed By Fluorescence Spectroscopy, Journal of Physical Chemistry B 101 (1997) 7635-7640.

[21] T. Heimburg, U. Würz, D. Marsh, Binary Phase Diagram of Hydrated Dimyristoylglycerolphophatidylcholine Mixtures, Biophysical Journal 63 (1992) 1369-1378.

[22] S. Matuoka, S. Kato, I. Hatta, Temperature Change of the Ripple Structure in Fully Hydrated Dimyristoylphosphatidylcholine/Cholesterol Multibilayers, Biophysical Journal 67 (1994) 728-736.

[23] T. Parasassi, M. Di Stefano, M. Loiero, G. Ravagnan, E. Gratton, Cholesterol Modifies Water Concentration and Dynamics in Phospholipid Bilayers: A Fluorescence Study Using Laurdan Probe, Biophysical Journal 66 (1994) 763768. 
[24] T. Parasassi, M. Di Stefano, M. Loiero, G. Ravagnan, E. Gratton, Influence of Cholesterol on Phospholipid Bilayers Phase Domains as Detected by Laurdan Fluorescence, Biophysical Journal 66 (1994) 120-132.

[25] D. P. Tieleman, S. J. Marrink, H. J. C. Berendsen, A Computer Perspective of Membranes: Molecular Dynamics Studies of Lipid Bilayer Systems, Biochimica et Biophysica Acta 1331 (1997) 235-270.

[26] S. Berneche, M. Nina, B. Roux, Molecular Dynamics Simulation of Melittin in a Dimyristoylphosphatidylcholine Bilayer Membrane, Biophysical Journal 75 (1998) 1603-1618.

[27] A. Tardieu, V. Luzzati, Stucture and Polymorphism of the Hydrocarbon Chains of Lipids: A Study of Lecithin-Water Phases, Journal of Molecular Biology 75 (1973) 711-733.

[28] H. Hauser, I. Pascher, R. H. Pearson, S. Sundell, Prefered Conformation and Molecular Packing of Phosphatidylethanolamine and Phosphatidylcholine, Biochimica et Biophysica Acta 1981 (1981) 21-51.

[29] R. N. A. H. Lewis, N. Mak, R. N. McElhaney, A Differential Scanning Calorimetric Study of the Thermotropic Phase Behavior of Model Membranes Composed of Phosphatidylcholines Containing Linear Saturated Fatty Acyl Chains, Biochemistry 26 (1987) 6118-6126.

[30] S. C. Chen, J. M. Sturtevant, B. J. Gaffney, Scanning Calorimetric Evidence for a third Phase Transition in Phosphatidylcholine Bilayers, Proceedings of the National Academy of Sciences of the United States of America 77 (1980) 5060-5063.

[31] S. Utoh, Split in Subtransition of Phospholipid Bilayer under High Pressure, Journal of Chemical Physics 106 (1997) 728-731.

[32] H. H. Füldner, Characterization of a Third Phase Transition in Multilamellar Dipalmitoyllecithin Liposomes, Biochemistry 20 (1981) 5707-5710.

[33] S. Utoh, N. Okubo, Subtransition of Multilamellar L- $\alpha$ Dibehenoyl $\left(C_{n}=22\right)$ Phosphatidylcholine, Japanese Journal of Applied Physics 40 (2001) 50195025.

[34] M. J. Ruocco, G. G. Shipley, Characterization of the Sub-Transition of Hydrated Dipalmitoylphosphatidylcholine Bilayers: Kinetics, Hydration and Structural Study, Biochimica et Biophysica Acta 691 (1982) 309-320. 
[35] M. J. Ruocco, G. G. Shipley, Characterization of the Sub-Transition of Hydrated Dipalmitoylphosphatidylcholine Bilayers: x-Ray Diffraction Study, Biochimica et Biophysica Acta 684 (1982) 59-66.

[36] I. Trahms, W. D. Klabe, E. Boroske, ${ }^{1}$ H-NMR Study of the Three Low Temperature Phases of DPPC-Water Systems, Biophysical Journal 42 (1983) 285-293.

[37] A. L. MacKay, A Proton NMR Moment Study of the Gel and Liquid-Crytalline Phases of Dipalmitoylphosphatidylcholine, Biophysical Journal 35 (1981) 301313.

[38] D. G. Cameron, H. L. Casal, E. F. Gudgin , H. H. Mantsch, The Gel Phase of Dipalmitoyl Phosphatidylcholine: An Infrared Characterization of the Acyl Chain Packing, Biochimica et Biophysica Acta 596 (1980) 463-467.

[39] E. Boroske, L. Trahms, $\mathrm{A}^{1} \mathrm{H}$ and ${ }^{13} \mathrm{C}$ NMR Study of Motional Changes of Dipalmitoyl Lecithin Associated wit the Pretransition, Biophysical Journal 42 (1983) 275-283.

[40] D. Marsh, Molecular Motion in Phospolipid Bilayers in Gel Phase: Long Axis Rotation, Biochemistry 19 (1980) 1632-1637.

[41] G. Cevc, How Membrane Chain-Melting Phase-Transition Temperature is Affected by the Lipid Chain Asymmetry and Degree of Unsaturation : An Effective Chain -Length Model, Biochemistry 30 (1991) 7186-7193.

[42] D. Marsh, Analysis of the Chainlength Dependence of Lipid Phase Transition Temperatures: Main and Pretransitions of Phosphatidylcholines: Main and Non-Lamellar Transitions of Phosphatidylethanolamines, Biochimica et Biophysica Acta 1062 (1991) 1-6.

[43] M. J. Janiak, D. M. Small, G. G. Shipley, Nature of the Thermal Pretransition of Synthetic Phospholipids: Dimyristoyl- and Dipalmitoyllecithin, Biochemistry 15 (1976) 4575-4580.

[44] M. J. Janiak, D. M. Small, G. G. Shipley, Temperature and Compositional Dependence of the Structure of Hydrated Dimyristoyl Lecithin, The Journal of Biological Chemistry 254 (1978) 6068-6078.

[45] Y. Inoko, T. Mitsui, Structural Parameters of Dipalmitoyl Phosphatidylcholine Lamellar Phases and Bilayer Phase Transitions, Journal of the Physical Society of Japan 44 (1978) 1918-1924. 
[46] B. A. Cunningham, A. D. Brown, D. H. Wolfe, W. P. Williams, A. Brain, Ripple Phase Formation in Phosphatidylcholine - Effect of Acyl Chain Relative Length, Position, and Unsaturation, Physical Review A 58 (1998) 3662-3672.

[47] M. F. Schneider, D. Marsh, W. Jahn, B. Kloesgen, T. Heimburg, Network Formation of Lipid Membranes: Triggering Structural Transitions by Chain Melting, Proceedings of the National Academy of Sciences of the United States of America 96 (1999) 14312-14317.

[48] J. Stamatoff, B. Feuer, H. J. Guggenheim, G. Tellez, T. Yamane, Amplitude of Rippling in the $P_{\beta}$ Phase of Dipalmitoylphosphatidylcholine Bilayers, Biophysical Journal 38 (1982) 217-226.

[49] R. P. Rand, D. Chapman, K. Larsson, Tilted Hydrocarbon Chains of Dipalmitoyl Lecithin become Perpendicular to the Bilayer before Melting, Biophysical Journal 15 (1975) 1117-1124.

[50] T. Le Bihan, M. Pezolet, Study of the Structure and Phase Behavior of Dipalmitoylphosphatidylcholine by Infrared Spectroscopy - Characterization of the Pretransition and Subtransition, Chemistry \& Physics of Lipids 94 (1998) 13-33.

[51] D. G. Cameron, H. L. Casal, H. H. Mantsch, Characterization of the Pretransition in 1,2-Dipalmitoyl-sn-glycero-3-phosphocholine by Fourier Transform Infrared Spectroscopy, Biochemistry 19 (1980) 3665-3672.

[52] D. G. Cameron, H. L. Casal, H. H. Mantsch, The Thermotropic Behavior of Dipalmitoyl Phosphatdylcholine Bilayers, Biophysical Journal 35 (1981) 1-16.

[53] J. F. Nagle, D. A. Wilkinson, Lecithin Bilayers Density Measurements and Molecular Interactions, Biophysical Journal 23 (1978) 159-175.

[54] D. W. McClure, Nature of the Rotational Phase Transition in Paraffin Crystals, Journal of Chemical Physics 49 (1968) 1830-1839.

[55] G. Cevc, Polymorphism of the Bilayer Membranes in the Orderd Phase and the Molecular Origin of the Lipid Pretransition and Rippled Lamellae, Biochimica et Biophysica Acta 1062 (1991) 59-69.

[56] M. Pasenkiewicz-Gierula, Y. Takaoka, H. Miyagawa, K. Kitamura, A. Kusumi, Hydrogen Bonding of Water to Phosphatidylcholine in the Membrane as Studied by a Molecular Dynamics Simulation - Location, Geometry, and LipidLipid Bridging via Hydrogen-Bonded Water, Journal of Physical Chemistry 101 (1997) 3677-3691. 
[57] M. Pasenkiewicz-Gierula, Y. Takaoka, H. Miyagawa, K. Kitamura, A. Kusumi, Charge Pairing of Headgroups in Phosphatidylcholine Membranes: A Molecular Dynamics Simulation Study, Biophysical Journal 76 (1999) 12281240.

[58] M. Pasenkiewicz-Gierula, T. Rog, Conformations, Orientations and Time Scales Characterising Dimyristoylphosphatidylcholine Bilayer Membrane Molecular Dynamics Simulation Studies, Acta Biochimica Polonica 44 (1997) 607-624.

[59] J. J. L. Cascales, H. J. C. Berendsen, J. G. Delatorre, Molecular Dynamics Simulation of Water between two Charged Layers of Dipalmitoylphosphatidylserine, Journal of Physical Chemistry 100 (1996) 8621-8627.

[60] I. Z. Zubrzycki, Y. Xu, M. Madrid, P. Tang, Molecular Dynamics Simulations of a Fully Hydrated Dimyristoylphosphatidylcholine Membrane in LiquidCrystalline Phase, Journal of Chemical Physics 112 (2000) 3437-3441.

[61] H. Heller, M. Schaefer, K. Schulten, Molecular Dynamics Simulation of a Bilayer of 200 Lipids in the Gel and the Liquid-Crystal Phases, Journal of Physical Chemistry 97 (1993) 8343-8360.

[62] Y. Takaoka, M. Pasenkiewicz-Gierula, H. Miyagawa, K. Kitamura, Y. Tamura, A. Kusumi, Molecular Dynamics Generation of Nonarbitrary Membrane Models reveals Lipid Orientational Correlations, Biophysical Journal 79 (2000) 31183138.

[63] F. Zhou, K. Schulten, Molecular Dynamics Study of a Membrane - Water Interface, Journal of Physical Chemistry 99 (1995) 2194-2207.

[64] E. H. Alper, D. Bassolino-Klimas, T. R. Stouch, The Limiting Behavior of Water Hydrating a Phospholipid Monolayer: A Computer Simulation Study, Journal of Chemical Physics 99 (1993) 5547-5559.

[65] E. H. Alper, D. Bassolino, T. R. Stouch, Computer Simulation of a Phospholipid Monolayer-Water System: The Influence of Long Forces on Water Structure and Dynamics, Journal of Chemical Physics 98 (1993) 97989807. 
[66] W. Shinoda, M. Shimizu, S. Okazaki, Molecular Dynamics Study on Electrostatic Properties of a Lipid Bilayer - Polarization, Electrostatic Potential, and the Effects on Structure and Dynamics of Water near the Interface, Journal of Physical Chemistry B 102 (1998) 6647-6654.

[67] R. Kubo, in D. T. Haar (Ed.): Fluctuation Relaxation and Resonance in Magnetic Systems, Oliver and Boyd, Edinburgh 1962, 23-68.

[68] R. Kubo, A Stochastic Theory of Line Shape, Advances in Chemical Physics 15 (1969) 101-127.

[69] R. Kubo, M. Toda, N. Hashitsume, Statistical Physics, Springer Verlag, Berlin 1991.

[70] J. L. Doob, The Brownian Movement and Stochastic Equations, Annals of Mathematics 43 (1942) 351-369.

[71] E. Hanamura, Stochastic Theory of Coherent Optical Transients, Journal of the Physical Society of Japan 52 (1983) 2258-2266.

[72] S. Mukamel, Principles of Nonlinear Optical Spectroscopy, Oxford University Press, New York 1995.

[73] S. Mukamel, V. Rupasov, Energy Transfer, Spectral Diffusion, and Fluorescence of Molecular Aggregates - Brownian Oscillator Analysis, Chemical Physics Letters 242 (1995) 17-26.

[74] W. P. de Boeij, M. S. Pshenichnikov, D. A. Wiersma, On the Relation Between the Echo-Peak Shift and Brownian-Oscillator Correlation Function, Chemical Physics Letters 253 (1996) 53-60.

[75] W. P. de Boeij, M. S. Pshenichnikov, D. A. Wiersma, System-Bath Correlation Function Probed By Conventional and Time-Gated Stimulated Photon Echo [Review], Journal of Physical Chemistry 100 (1996) 11806-11823.

[76] W. P. de Boeij, Ultrafast Solvation Dynamics Explored by Nonlinear Optical Spectroscopy, Dissertation, Groningen 1997.

[77] K. F. Everitt, E. Geva, J. L. Skinner, Determining the Solvation Correlation Function from Three-Pulse Photon Echoes in Liquids, Journal of Chemical Physics 114 (2001) 1326-1335.

[78] R. Agarwal, B. P. Krueger, G. D. Scholes, M. Yang, J. Yom, L. Mets, G. R. Fleming, Ultrafast Energy Transfer in LHC-II Revealed by Three-Pulse Photon Echo Peak Shift Measurements, Journal of Physical Chemistry B 104 (2000) 2908-2918. 
[79] X. J. Jordanides, M. J. Lang, X. Y. Song, G. R. Fleming, Solvation Dynamics in Protein Environments Studied by Photon Echo Spectroscopy, Journal of Physical Chemistry B 103 (1999) 7995-8005.

[80] B. J. Homoelle, M. D. Edington, W. M. Diffey, W. F. Beck, Stimulated PhotonEcho and Transient-Grating Studies of Protein-Matrix Solvation Dynamics and Interexciton-State Radiationless Decay in $\alpha$-Phycocyanin and Allophycocyanin, Journal of Physical Chemistry B 102 (1998) 3044-3052.

[81] T. H. Joo, Y. W. Jia, J. Y. Yu, M. J. Lang, G. R. Fleming, Third-Order Nonlinear Time Domain Probes of Solvation Dynamics [Review], Journal of Chemical Physics 104 (1996) 6089-6108.

[82] E. J. Brown, Q. G. Zhang, M. Dantus, Femtosecond Transient-Grating Techniques: Population and Coherence Dynamics Involving Ground and Excited States [Review], Journal of Chemical Physics 110 (1999) 5772-5788.

[83] C. Kittel, Einführung in die Festkörperphysik, München, Wien 1989.

[84] S. Backus, C. G. Durfee, M. M. Murnane, H. C. Kapteyn, High Power Ultrafast Lasers, Review of Scientific Instruments 69 (1998) 1207-1223.

[85] S. Backus, J. Peatross, C. P. Huang, M. M. Murnane, H. C. Kapteyn, Ti:Sapphire Amplifier Producing Millijoule-Level, 21-fs Pulses at $1 \mathrm{kHz}$, Optics Letters 20 (1995) 2000-2002.

[86] P. Maine, D. Strickland, P. Bado, M. Pessot, G. Mourou, Generation of Ultrahigh Peak Power Pulses by Chirped Pulse Amplification, IEEE Journal of Quantum Electronics 24 (1988) 398-403.

[87] G. A. Mourou, C. P. J. Barty, M. D. Perry, Ultrahigh-Intensity Lasers: Physics of the Extreme on a Tabletop, Physics Today 51 (1998) 22-28.

[88] J. Zhou, C.-P. Huang, M. M. Murnane, H. C. Kapteyn, Amplification of 26-fs, 2TW Pulses near the Gain-Narrowing Limit in Ti:Sapphire, Optics Letters 20 (1995) 64-66.

[89] G. Lenz, K. Tamura, H. A. Haus, E. P. Ippen, All-Solid-State Femtosecond Source at $1.55 \mu \mathrm{m}$, Optics Letters 20 (1995) 1289-1291.

[90] L. E. Neslon, D. J. Jones, K. Tamura, H. A. Haus, E. P. Ippen, UltrashortPulse Fiber Ring Lasers, Applied Physics B-Lasers \& Optics 65 (1997) 277294.

[91] K. Tamura, E. P. Ippen, H. A. Haus, Pulse Dynamics in Streched-Pulse Fiber Lasers, Applied Physics Letters 67 (1995) 158-160. 
[92] Z. Bor, B. Racz, Group Velocity Dispersion in Prisms and its Application to Pulse Compression and Travelling-Wave Excitation, Optics Communications 54 (1985) 165-170.

[93] R. L. Fork, O. E. Martinez, J. P. Gordon, Negative Dispersion Using Pairs of Prisms, Optics Letters 9 (1984) 150-152.

[94] E. B. Treacy, Optical Pulse Compression with Diffraction Gratings, IEEE Journal of Quantum Electronics QE-5 (1969) 454-458.

[95] G. Cerullo, M. Nisoli, S. Stagira, S. Desilvestri, Sub-8-Fs Pulses From an Ultrabroadband Optical Parametric Amplifier in the Visible, Optics Letters 23 (1998) 1283-1285.

[96] G. Cerullo, M. Nisoli, S. Desilvestri, Generation of 11 Fs Pulses Tunable Across the Visible by Optical Parametric Amplification, Applied Physics Letters 71 (1997) 3616-3618.

[97] J. Ringling, O. Kittelmann, F. Noack, G. Korn, J. Squier, Tunable Femtosecond Pulses in the near Vacuum Ultraviolet Generated by Frequency Conversion of Amplified Ti:Sapphire Laser Pulses, Optics Letters 18 (1993) 2035-2037.

[98] A. Shirakawa, I. Sakane, T. Kobayashi, Pulse-Front-Matched Optical Parametric Amplification for Sub-10-Fs Pulse Generation Tunable in the Visible and Near Infrared, Optics Letters 23 (1998) 1292-1294.

[99] A. Shirakawa, I. Sakane, M. Takasaka, T. Kobayashi, Sub-5-fs Visible Pulse Generation by Pulse-Front-Matched Noncollinear Optical Parametric Amplification, Applied Physics Letters 74 (1999) 2268-2270.

[100] T. Kobayashi, A. Shirakawa, Tunable Visible and Near-Infrared Pulse Generator in a 5 fs Regime, Applied Physics B-Lasers \& Optics 70 (2000) 239246.

[101] W. Demtröder, Laserspektroskopie, Springer, Berlin 1991.

[102] Y. R. Shen, The Principles of Nonlinear Optics, New York 1984.

[103] A. Andreoni, M. Bondani, Group-Velocity Control in the Mixing of Three Noncollinear Phase-Matched Waves, Applied Optics 37 (1998) 2414-2423.

[104] N. Boeuf, D. Branning, I. Chaperot, E. Dauler, S. Guerin, G. Jaeger, A. Muller, A. Migdall, Calculating Characteristics of Noncollinear Phase Matching in Uniaxial and Biaxial Crystals, Optical Engineering 39 (2000) 1016-1024. 
[105] A. Shirakawa, T. Kobayashi, Noncollinearly Phase-Matched Femtosecond Optical Parametric Amplification with a $2000 \mathrm{~cm}^{-1}$ Bandwidth, Applied Physics Letters 72 (1998) 147-149.

[106] P. Di Trapani, A. Andreoni, C. Solcia, P. Foggi, R. Danielius, A. Dubietis, A. Piskarskas, Matching of Group Velocities in Three-Wave Parametric Interaction With Femtosecond Pulses and Application to Traveling-Wave Generators, Journal of the Optical Society of America B-Optical Physics 12 (1995) 2237-2244.

[107] P. Di Trapani, A. Andreoni, G. P. Banfi, C. Solcia, R. Danielius, A. Piskarskas, P. Foggi, M. Monguzzi, C. Sozzi, Group-Velocity Self-Matching of Femtosecond Pulses in Noncollinear Parametric Generation, Physical Review A 51 (1995) 3164-3168.

[108] T. Wilhelm, J. Piel, E. Riedle, Sub-20-Fs Pulses Tunable Across the Visible from a Blue-Pumped Single-Pass Noncollinear Parametric Converter, Optics Letters 22 (1997) 1494-1496.

[109] E. Riedle, M. Beutter, S. Lochbrunner, J. Piel, S. Schenkl, S. Sporlein, W. Zinth, Generation of 10 to 50 fs Pulses Tunable Through all of the Visible and the NIR, Applied Physics B-Lasers \& Optics 71 (2000) 457-465.

[110] J. Piel, M. Beutter, E. Riedle, 20-50-fs Pulses Tunable across the Near Infrared from a Blue-Pumped Noncollinear Parametric Amplifier, Optics Letters 25 (2000) 180-182.

[111] R. R. Alfano, The Supercontinuum Laser Source, New York 1989.

[112] HewlettPackard, VEE OneLab: 6.0.

[113] D. C. Arnett, Ultrafast Coherence Studies of the First Events in Photosynthesis, Dissertation, Pennsylvania 1997.

[114] T. MathWorks, Matlab: 6.0.0.88.

[115] D. E. Wolf, in L. M. Loew (Ed.): Spectroscopic Membrane Probes, Vol. 1, CRC Press, Boca Raton,Fla USA 1988, 193-220.

[116] D. Axelrod, Carbocyanine Dye Orientation in Red Cell Membrane Studied by Microscopic Fluorescence Polarization, Biophysical Journal 26 (1979) 557574.

[117] D. E. Wolf, Determination of the Sidness of Carbocyanine Dye Labeling of Membranes, Biochemistry 24 (1985) 582-586. 
[118] E. E. Jelley, Spectral Absorption and Fluorescence of Dyes in the Molecular State, Nature 138 (1936) 1009-1010.

[119] G. Scheibe, Über die Veränderlichkeit der Absorptionsspektren in Lösung und die van der Waalsschen Kräfte als ihre Ursache, Angewandte Chemie 50 (1937) 51.

[120] H. Fidder, J. Terpstra, D. A. Wiersma, Dynamics of Frenkel Excitons in Disordered Molecular Aggregates, Journal of Chemical Physics 94 (1991) 6895-6907.

[121] E. O. Potma, D. A. Wiersma, Exciton Superradiance in Aggregates - the Effect of Disorder, Higher Order Exciton-Phonon Coupling and Dimensionality, Journal of Chemical Physics 108 (1998) 4894-4903.

[122] R. Koynova, M. Caffrey, Phases and Phase Transitions of the Phosphatidylcholines [Review], Biochimica et Biophysica Acta - Reviews on Biomembranes 1376 (1998) 91-145.

[123] T. Heimburg, Mechanical Aspects of Membrane Thermodynamics. Estimation of the Mechanical Properties of Lipid Membranes close to the Chain Melting Transition from Calorimetry, Biochimica et Biophysica Acta - Biomembranes 1415 (1998) 147-162.

[124] C. H. Spink, M. D. Yeager, G. W. Feigenson, Partitioning Behavior of Indocarbocyanine Probes between Coexisting Gel and Fluid Phases in Model Membranes, Biochimica et Biophysica Acta 1023 (1990) 25-33.

[125] R. D. Klausner, D. E. Wolf, Selectivity of Fluorescent Lipid Analogues of Lipid Domains, Biochemistry 19 (1980) 6199-6203.

[126] M. F. Ethier, D. E. Wolf, D. L. Melchior, Calorimetric Investigation of the Phase Partitioning of the Fluorescent Carbocyanine Probes in Phosphatidylcholine Bilayers, Biochemistry 22 (1983) 1178-1182.

[127] L. Bergmann, C. Schaefer, Lehrbuch der Experimentalphysik, Optik, Vol. 3, Walter de Gruyter, Berlin, New York 1987.

[128] R. Lipowsky, E. Sackmann, Structur and Dynamics of Membranes: Generic and Specific Interactions, Vol. 1B, Amsterdam 1995.

[129] H. Sato, M. Kawasaki, K. Kasatani, K. M., Raman Spektra of Some Indo-, Thia- and Selena-carbocyanine Dyes, Journal of Raman Spectroscopy 19 (1988) 129-132. 
[130] K. Iwata, W. L. Weaver, T. L. Gustafson, Spontaneous Raman Spectra of the Cyanine Dye DODCl and its Six Analogues using Titanium:Sapphire Laser Excitation, Journal of Physical Chemistry 96 (1992) 10219-10224.

[131] J.-P. Yang, R. H. Callender, The Resonance Raman Spectra of some Cyanine Dyes, Journal of Raman Spectroscopy 16 (1985) 319-321.

[132] M. Katsumata, K. Kasatani, M. Kawasaki, H. Sato, Highly Aggregated State of the Dye with the Detergent in the Premicellar Region by Resonance Raman Spectra, Bulletin of the Chemical Society of Japan 55 (1982) 717-720.

[133] B. S. Packard, D. E. Wolf, Fluorescence Lifetime of Carbocyanine Lipid Analogues in Phospholipid Bilayers, Biochemistry 24 (1985) 5176-5181.

[134] D. Zhifei, Photophysics of Indocarbocyanine Dyes in Organic Solvents, Journal of the Chinese Chemical Society 45 (1998) 237-240.

[135] S. A. Passino, Y. Nagasawa, T. Joo, G. R. Fleming, Three-Pulse Echo Peak Shift Studies of Polar Solvation Dynamics, Journal of Physical Chemistry 101 (1997) 725-731.

[136] S. A. Passino, Y. Nagasawa, G. R. Fleming, Three Pulse Stimulated Photon Echo Experiments as a Probe of Polar Solvation Dynamics - Utility of Harmonic Bath Models, Journal of Chemical Physics 107 (1997) 6094-6108.

[137] C. J. Bardeen, S. J. Rosenthal, C. V. Shank, Ultrafast Solvation Processes in Polar Liquids Probed with Large Organic Molecules, Journal of Physical Chemistry 103 (1999) 10506-10516.

[138] D. Pant, N. E. Levinger, Polar Solvation Dynamics of $\mathrm{H}_{2} \mathrm{O}$ and $\mathrm{D}_{2} \mathrm{O}$ at the Surface of Zirconia Nanoparticles, Journal of Physical Chemistry B 103 (1999) 7846-7852.

[139] M. Maroncelli, The Dynamics of Solvation in Polar Liquids, Journal of Molecular Liquids 57 (1993) 1-37.

[140] E. W. J. Castner, G. R. Fleming, B. Bagchi, M. Maroncelli, The Dynamics of Polar Solvation: Inhomogeneous Dielectric Continuum Models, Journal of Chemical Physics 89 (1988) 3519-3534.

[141] H. Bürsing, D. Ouw, S. Kundu, P. Vöhringer, Probing Solvation Dynamics in Liquid Water and at Phospholipid/Water Interfaces with Femtosecond PhotonEcho Spectroscopies, Physical Chemistry Chemical Physics 3 (2001) 23782387. 
[142] G. S. Beddard, T. Doust, G. Porter, Picosecond Fluorescence Depolarisation Measured by Frequency Conversion, Chemical Physics 61 (1981) 17-23.

[143] C. V. Shank, E. P. Ippen, Anisotropic Absorption Saturation with Picosecond Pulses, Applied Physics Letters 26 (1975) 62-63.

[144] P. R. Mason, J. B. Hasted, L. Moore, The Use of Statistical Theory in Fitting Equations to Dielectric Disperion Data, Advances in Moleculaar Relaxataion Processes 6 (1974) 217-232.

[145] E. Lang, H.-D. Lüdemann, Pressure and Temperature Dependence of the Longitudinal Proton Relaxation Times in Supercooled Water to $-87^{\circ} \mathrm{C}$ and 2500 bar, Journal of Chemical Physics 67 (1977) 718-720.

[146] E. Lang, H.-D. Lüdemann, Pressure and Temperature Dependence of the Longitudinal Deuterium Relaxation Times in Supercooled Heavy Water to 300 MPa and $188 \mathrm{~K}$, Berichte der Bunsen Gesellschaft fur Physikalische ChemieAn International Journal of Physical Chemistry 84 (1980) 462-470.

[147] E. W. Lang, H.-D. Lüdemann, Anomalies of Liquid Water, Angewandte Chemie 21 (1982) 315-388.

[148] C. Ronne, L. Thrane, P. O. Astrand, A. Wallqvist, K. V. Mikkelsen, S. R. Keiding, Investigation of the Temperature Dependence of Dielectric Relaxation in Liquid Water by $\mathrm{THz}$ Reflection Spectroscopy and Molecular Dynamics Simulation, Journal of Chemical Physics 107 (1997) 5319-5331.

[149] C. Ronne, P. O. Astrand, S. R. Keiding, THz spectroscopy of Liquid $\mathrm{H}_{2} \mathrm{O}$ and $\mathrm{D}_{2} \mathrm{O}$, Physical Review Letters 82 (1999) 2888-2891.

[150] K. Winkler, J. Lindner, H. Bürsing, P. Vöhringer, Ultrafast Raman-Induced Kerr-Effect of Water: Single Molecule versus Collective Motions, Journal of Chemical Physics 113 (2000) 4674-4682.

[151] P. Foggi, M. Bellini, D. P. Kien, I. Vercuque, R. Righini, Relaxation Dynamics of Water and $\mathrm{HCl}$ Aqueous Solutions Measured by Time-Resolved Optical Kerr Effect, Journal of Physical Chemistry 101 (1997) 7029-7035.

[152] V. Mazzacurati, A. Nucara, M. A. Ricci, G. Ruocco, G. Signorelli, HighResolution Low-Frequency Raman Spectra of Liquid $\mathrm{H}_{2} \mathrm{O}$ and $\mathrm{D}_{2} \mathrm{O}$, Journal of Chemical Physics 93 (1990) 7767-7773.

[153] G. E. Walrafen, Raman Spectrum of Water: Transverse and Longitudinal Acoustic Modes below $300 \mathrm{~cm}^{-1}$ and Optic Modes above $300 / \mathrm{cm}$, Journal of Physical Chemistry 94 (1990) 2237-2239. 
[154] M. J. Lang, X. J. Jordanides, X. Song, G. R. Fleming, Aqueous Solvation Dynamics Studied by Photon Echo Spectroscopy, Journal of Chemical Physics 110 (1999) 5884-5892.

[155] Y. Nagasawa, S. A. Passino, T. Joo, G. R. Fleming, Temperature Dependence of Optical Dephasing in an Organic Polymer Glass (PMMA) from $300 \mathrm{~K}$ to 30 K, Journal of Chemical Physics 106 (1997) 4840-4852.

[156] R. Jimenez, G. R. Fleming, P. V. Kumar, M. Maroncelli, Femtosecond Solvation Dynamics in Water, Nature 369 (1994) 471-473.

[157] M. Maroncelli, G. R. Fleming, Comparison of Time-Resolved Fluorescence Stokes Shift Measurements to a Molecular Theory of Solvation Dynamics, Journal of Chemical Physics 89 (1988) 875-881.

[158] J. S. Bader, D. Chandler, Computer Simulation of Phosphochemically Induced Electron Transfer, Chemical Physics Letters 157 (1989) 501-504.

[159] X. Y. Song, D. Chandler, Dielectric Solvation Dynamics of Molecules of Arbitrary Shape and Charge Distribution, Journal of Chemical Physics 108 (1998) 2594-2600.

[160] B. Zolotov, A. Gan, B. D. Fainberg, D. Huppter, Resonance Heterodyne Optical Kerr Spectroscopy of Solvation Dynamics in Water and $\mathrm{D}_{2} \mathrm{O}$, Chemical Physics Letters 265 (1997) 418-426.

[161] H. Barkhuijsen, R. de Beer, W. M. M. J. Bovee, D. van Ormondt, Retrieval of Frequencies, Amplitude, Damping Factors, and Phases from Time-Domain Signals Using Linear Least-Squares Procedure, Journal of Magnetic Resonance 61 (1985) 465-481.

[162] H. Barkhuijsen, R. de Beer, D. van Ormondt, Improved Algorithm of Noniterative Time-Domain Model Fitting to Exponentially Damped Magnetic Resonance Signals, Journal of Magnetic Resonance 73 (1987) 553-557.

[163] W. Jarzeba, G. C. Walker, A. E. Johnson, M. A. Kahlow, P. F. Barbara, Femtosecond Microscopic Solvation Dynamics of Aqueous Solutions, Journal of Physical Chemistry 92 (1988) 7039-7041.

[164] C. P. Hsu, X. Y. Song, R. A. Marcus, Time-Dependent Stokes Shift and Its Calculation from Solvent Dielectric Dispersion Data, Journal of Physical Chemistry B 101 (1997) 2546-2551. 
[165] H. L. Friedman, Fast Response of a Dielectric to the Motion of a Charge, Journal of the Chemical Society, Faraday transactions 279 (1983) 1465-1467.

[166] D. Kivelson, H. Friedman, Longitudinal Dielectric Relaxation, Journal of Physical Chemistry 93 (1989) 7026-7031.

[167] M. Maroncelli, Computer Simulations of Solvation Dynamics in Acetonitrile, Journal of Chemical Physics 94 (1991) 2084-2103.

[168] M. Maroncelli, Continuum Estimates of Rotational Dielectric Friction and Polar Solvation, Journal of Chemical Physics 106 (1997) 1545-1555.

[169] X. Song, R. A. Marcus, Quantum Correction for Electron Transfer Rates. Comparison of Polarizable versus Nonpolarizable Descriptions of Solvent, Journal of Chemical Physics 99 (1993) 7768-7773.

[170] X. Y. Song, D. Chandler, R. A. Marcus, Gaussian Field Model of Dielectric Solvation Dynamics, Journal of Physical Chemistry 100 (1996) 11954-11959.

[171] G. R. Fleming, J. M. Morris, G. W. Robinson, Direct Observation of Rotational Diffusion by Picosecond Spectroscopy, Chemical Physics 17 (1976) 91-100.

[172] R. M. Lynden-Bell, I. R. McDonald, Reorientational Correlation Functions for Computer-Simulated Liquids of Tetrahedral Molecules, Molecular Physics 43 (1981) 1429-1440.

[173] R. M. Lynden-Bell, I. R. McDonald, Tests of Models of Molecular Reorientation in Liquids, Chemical Physics Letters 89 (1982) 105-109.

[174] R. M. Lynden-Bell, W. A. Steele, A Model for Strongly Hindered Molecular Reorientation in Liquids, Journal of Physical Chemistry 88 (1984) 6514-6518.

[175] O. Conde, J. Teixeira, Hydrogen Bond Dynamics in Water Studied by Depolarized Rayleigh Scattering, Journal de physique 44 (1983) 525-529.

[176] O. Conde, J. Teixeira, Depolarized Light Scattering of Heavy Water, and Hydrogen Bond Dynamics, Molecular Physics 53 (1984) 951-959.

[177] K. Winkler, Ultraschneller Optischer Kerr-Effekt von Wasser und schwerem Wasser, Diplom, Göttingen 2000.

[178] G. Weber, F. J. Farris, Synthesis and Spectral Properties of a Hydrophobic Fluorescent Probe: 6-Propionyl-2-(dimehtylamino)naphthalene, Biochemistry 18 (1979) 3075-3078.

[179] P. L.-G. Chong, Effects of Hydrostatic Pressure on the Location of Prodan in Lipid Bilayers and Cellular Membranes, Biochemistry 27 (1988) 399-404. 
[180] P. L.-G. Chong, S. Capes, P. T. T. Wong, Effects of Hydrostatic Pressure on the Location of Prodan in Lipid Bilayers: A FT-IR Study, Biochemistry 28 (1989) 8358-8363.

[181] A. Sommer, F. Paltauf, A. Hermetter, Dipolar Solvent Relaxation on a Nanosecond Time Scale in Ether Phospholipid Membranes as Determined by Multifrequency Phase and Modulation Fluorometry, Biochemistry 29 (1990) 11134-11140.

[182] H. Rottenberg, Probing the Interactions of Alcohols with Biological Membranes with the Fluorescent Probe Prodan, Biochemistry 31 (1992) 9473-9481.

[183] E. K. Krasnowska, E. Gratton, T. Parasassi, Prodan as a Membrane Surface Fluorescence Probe - Partitioning Between Water and Phospholipid Phases, Biophysical Journal 74 (1998) 1984-1993.

[184] T. Parasassi, M. Di Stefano, G. Ravagnan, R. M. Rusch, E. Gratton, Quantitation of Lipid Phases in Phospholipid Vesicles by the Generalized Polarization of Laurdan Fluorescence, Biophysical Journal 60 (1991) 179-189.

[185] M. Viard, J. Gallay, M. Vincent, M. Paternostre, Origin of Laurdan Sensitivity to the Vesicle-to-Micelle Transition of Phospholipid-Octylglucoside System: A Time-Resolved Fluorescence Study, Biophysical Journal 80 (2001) 347-359.

[186] T. Parasassi, E. K. Krasnowska, L. Bagatolli, E. Gratton, Laurdan and Prodan as polarity-sensitive fluorescent membrane probes, Journal of Fluorescence 8 (1998) 365-373.

[187] L. A. Bagatolli, T. Parasassi, G. D. Fidelio, E. Gratton, A Model for the Interaction of 6-Lauroyl-2-(N,N-dimethylamino)naphthalene with Lipid Environments: Implications for Spectral Properties, Journal of Photochemistry and Photobiology A: Chemie 70 (1999) 557-564.

[188] N. Nandi, B. Bagchi, Dielectric Relaxation of Biological Water, Journal of Physical Chemistry B 101 (1997) 10954-10961.

[189] W. P. de Boeij, M. S. Pshenichnikov, D. A. Wiersma, Ultrafast Solvation Dynamics Explored By Femtosecond Photon Echo Spectroscopies [Review], Annual Review of Physical Chemistry 49 (1998) 99-123. 


\section{DANKSAGUNG}

Besonders herzlich danke ich Herrn Priv. Doz. Dr. Peter Vöhringer für die ausgezeichneten Arbeitsbedingungen in seiner Gruppe. Seine Begeisterung für das Projekt hat maßgeblich zum Gelingen dieser Arbeit beigetragen. Ohne seine wissenschaftliche Unterstützung wären die hier vorgestellten Ergebnisse nicht in dieser Form zustande gekommen.

Herrn Dr. Jörg Lindner danke ich besonders für die konstruktive Unterstützung in wissenschaftlichen Fragen.

Herrn Dr. Santi Kundu danke ich für die Zusammenarbeit bei den Experimenten.

Herrn Dipl. Chem. Marco Seidel und Frau Dipl. Chem. Kathrin Winkler möchte ich besonders für die gute Stimmung und die hervorragende Zusammenarbeit im Labor danken. Die Diskussionen über immer neue weltbewegende Themen machten es unmöglich, den Humor zu verlieren.

Besonders danke ich den Doktoranden Nicole Hartmann, Kathrin Winkler und Marco Seidel sowie Dr. Jörg Lindner diese Arbeit Korrektur gelesen zu haben.

Herrn Eur.-Ing. Jaydev Jethwa danke ich für seine spontane Hilfe. Sein Vorrat an elektronischen Geräten scheint unerschöpflich zu sein.

Frau Eveline Heinemann und Herrn Dr. Matthias Müller danke ich für die sportliche Herausforderung in den Mittagspausen.

Weiterhin danke ich allen Mitarbeitern der Werkstätten, die sehr zum Gelingen dieser Arbeit beigetragen haben. Herausheben möchte ich Herrn Wolfgang Sauermann, der innerhalb kürzester Zeit in der Lage war, optische Komponenten bereitzustellen und so das durchführen der Experimente beschleunigt hat.

Mein besonderer Dank gilt der Arbeitsgruppe von Dr. Thomas Heimburg, die mich bei der Präparation der Vesikel unterstützt hat.

Besonders danke ich meinen Eltern für die moralische und kulinarische Unterstützung.

Nicht zuletzt danke ich meiner Frau Diana und meiner süßen Tochter Jule dafür, dass sie mich in der Zeit des Zusammenschreibens in Liebe ertragen und mit noch mehr Liebe unterstützt haben. 


\section{LEBENSLAUF}

Am 12. Juli 1972 wurde ich, Helge Bürsing, als Sohn des Finanzkaufmanns Helmut Bürsing und seiner Frau Christa Bürsing, geb. Baumeister, in Gifhorn geboren.

In den Jahren 1979 bis 1983 besuchte ich die Grundschule und anschließend bis 1985 die Orientierungsstufe in Celle. Im Sommer 1987 wechselte ich auf das Gymnasium Ernestinum.

In der Zeit von Juli 1989 bis Juni 1990 besuchte ich die High School der La Fargeville Central School im Staat New York der Vereinigten Staaten von Amerika und erzielte einen erfolgreichen Abschluss.

Meine Schulbildung setzte ich anschließend am Gymnasium Ernestinum in Celle fort, wo ich im Führjahr 1993 das Zeugnis der allgemeinen Hochschulreife erlangte.

Von Juli 1993 bis Juni 1994 leistete ich meinen Grundwehrdienst in einer Heeresfliegerstaffel in Celle ab.

Zum Wintersemester 1994/95 immatrikulierte ich mich an der Georg-AugustUniversität in Göttingen im Diplomstudiengang Chemie und bestand am Anfang des Wintersemesters 1996/97 die Diplomvorprüfung.

Im Frühjahr 1998 begann ich unter der Leitung von Priv. Doz. Peter Vöhringer am Max-Planck-Institut für biophysikalische Chemie in Göttingen meine Diplomarbeit mit dem Titel „Femtosekundenspektroskopie zur Photodissoziation von Quecksilberdiiodid in Lösung“. Diese Arbeit schloss ich im Mai 1999 mit der Diplomprüfung ab.

Seitdem beschäftige ich mich als wissenschaftliche Mitarbeiter am Max-PlanckInstitut für biophysikalische Chemie in Göttingen mit der Solvatationsdynamik an Lipidmembran-Wasser-Grenzschichten. Die Ergebnisse dieser Untersuchungen sind in dieser Arbeit zusammengefasst.

Seit dem 8. Juli 1999 bin ich mit der Erzieherin Diana Bürsing, geb. Wentzel, verheiratet., am 24. Juli 2001 wurde unsere Tochter Jule geboren. 